\title{
CAN PRESELECTION GAZE DISTRIBUTION STATISTICS PREDICT GRAPHICAL PASSWORDS?
}

\author{
A thesis submitted to \\ The Faculty of Graduate Studies and Research \\ In Partial Fulfillment of the requirements for the degree
}

Master of Arts

by

Daniel LeBlanc

Department of Psychology

Carleton University

January 2009

(C) Daniel LeBlanc 


$\begin{array}{ll}\begin{array}{l}\text { Library and } \\ \text { Archives Canada }\end{array} & \begin{array}{l}\text { Bibliothèque et } \\ \text { Archives Canada }\end{array} \\ \begin{array}{l}\text { Published Heritage } \\ \text { Branch }\end{array} & \begin{array}{l}\text { Direction du } \\ \text { Patrimoine de l'édition }\end{array} \\ \begin{array}{l}\text { 395 Wellington Street } \\ \text { Ottawa ON K1A 0N4 } \\ \text { Canada }\end{array} & \begin{array}{l}\text { 395, rue Wellington } \\ \text { Ottawa ON K1A 0N4 } \\ \text { Canada }\end{array}\end{array}$

Your file Votre référence ISBN: 978-0-494-47500-3

Ourfile Notre référence

ISBN: 978-0-494-47500-3

NOTICE:

The author has granted a nonexclusive license allowing Library and Archives Canada to reproduce, publish, archive, preserve, conserve, communicate to the public by telecommunication or on the Internet, loan, distribute and sell theses worldwide, for commercial or noncommercial purposes, in microform, paper, electronic and/or any other formats.

The author retains copyright ownership and moral rights in this thesis. Neither the thesis nor substantial extracts from it may be printed or otherwise reproduced without the author's permission.
AVIS:

L'auteur a accordé une licence non exclusive permettant à la Bibliothèque et Archives Canada de reproduire, publier, archiver, sauvegarder, conserver, transmettre au public par télécommunication ou par l'Internet, prêter, distribuer et vendre des thèses partout dans le monde, à des fins commerciales ou autres, sur support microforme, papier, électronique et/ou autres formats.

L'auteur conserve la propriété du droit d'auteur et des droits moraux qui protège cette thèse. $\mathrm{Ni}$ la thèse ni des extraits substantiels de celle-ci ne doivent être imprimés ou autrement reproduits sans son autorisation.
In compliance with the Canadian Privacy Act some supporting forms may have been removed from this thesis.

While these forms may be included in the document page count, their removal does not represent any loss of content from the thesis.
Conformément à la loi canadienne sur la protection de la vie privée, quelques formulaires secondaires ont été enlevés de cette thèse.

Bien que ces formulaires aient inclus dans la pagination, il n'y aura aucun contenu manquant.

\section{Canada}




\begin{abstract}
Graphical passwords are a new method of authentication of much interest to researchers due to the potential for better usability and security. This thesis first introduces past research on usability and security issues relating to graphical passwords. The thesis then presents a study to investigate a possible weakness in a particular approach to click-based graphical passwords, whether eye gaze information may assist attackers in guessing passwords. An experiment was conducted using a graphical password software application and an eye tracker. The experiment tested whether gaze patterns from a small set of participants resembled user click patterns in password choices. The gaze patterns did resemble the click point patterns, so gaze data would offer attackers an advantage over guessing at random. Gaze data could potentially be gathered without explicit interaction by participants, suggesting this could pose an attractive strategy for attackers.
\end{abstract}




\section{Acknowledgements}

Words are difficult to find to express my gratitude to my M.A. supervisor, Dr. Robert Biddle. His enthusiasm, his inspiration, and his great efforts to explain things clearly and simply motivated me to work diligently and efficiently; in short, this thesis would not have been possible without him.

I would like to thank the committee members, Dr. Andrew Patrick, Dr. Paul Van Oorschot, Dr. Brian Tansley, and Dr. Timothy Pychyl for their continued support throughout the writing process. I would like to extend these thanks to my colleagues at the HO'T Lab, who helped me throughout the usability testing phases of my research, and with this answered countless questions; I thank them for their patience and understanding.

To my fiancée, having never known me as anything else than a student, who has stood by me through thick and thin for the last six years. I love you, Jacynthe.

I would like to extend a very special thank you to my loving family. They raised me, supported me, taught me, and loved me. To them I dedicate this thesis. 


\section{Table of Contents}

Abstract..................................................................................

Acknowledgements...............................................................iii

Introduction .....................................................................

Graphical Passwords....................................................................

Advantages of Graphical Passwords over Text-based Passwords..................................5

Attacks on Text-based Passwords and Graphical Passwords................................ 10

Research Focus................................................................. 11

Main Research Question............................................................12

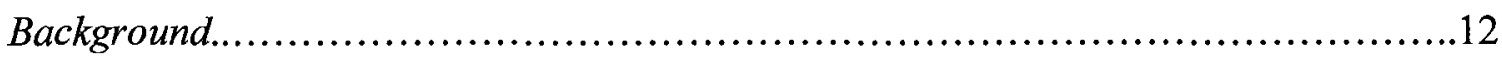

The Usability of Graphical Passwords................................................. 12

The Security of Graphical Passwords...................................................16

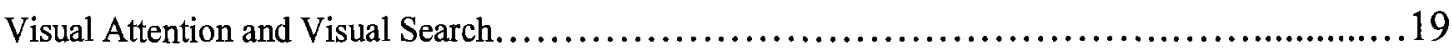

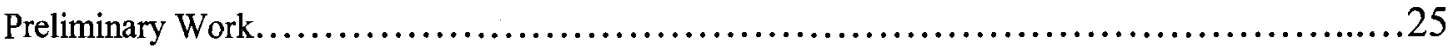

Research Study .........................................................29

Research Hypotheses.........................................................29

Method.................................................................. 30

Click-Points Data.............................................................

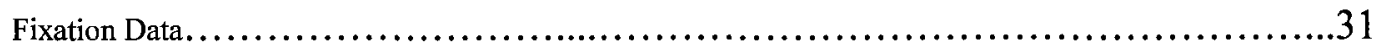

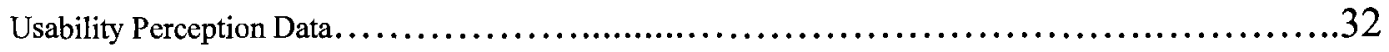

Equipment........................................................... 32

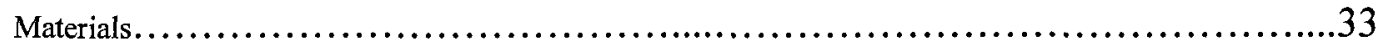

Participants.......................................................... 37

Procedure............................................................ 37

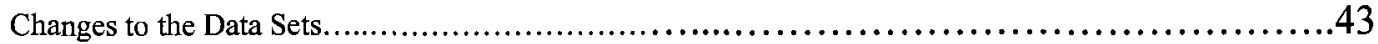

User Feedback Data........................................................43

Analysis.......................................................................44

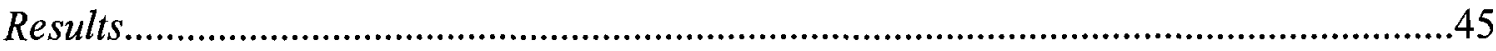

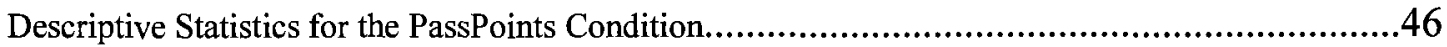

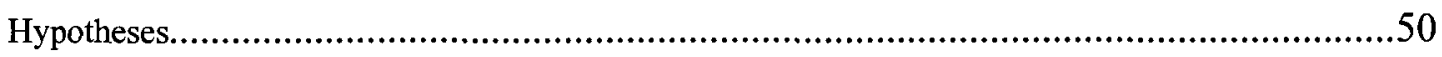

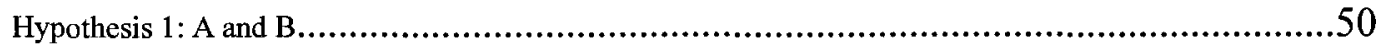




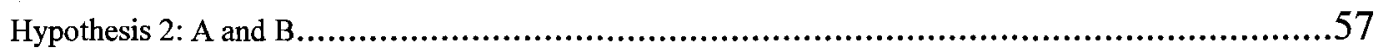

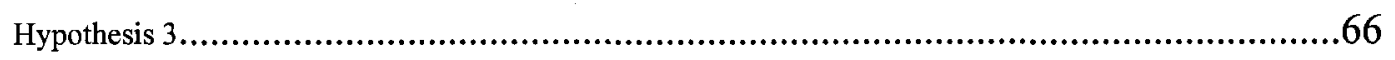

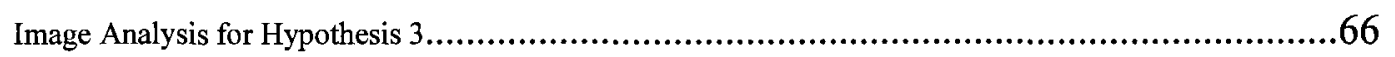

Hypothesis 3 Summary: Image Analysis for New Clicks and Gaze Data....................81

Hypothesis 3 Summary: Image Analysis for Old Clicks, Field Clicks, and Gaze Data..............81

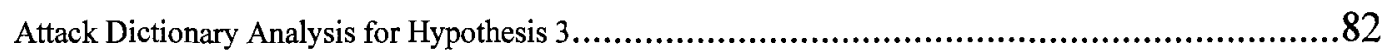

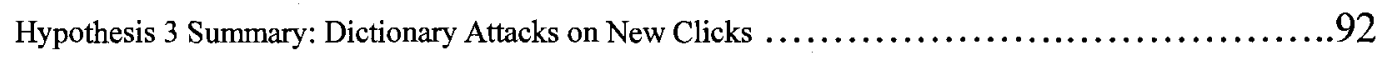

Hypothesis 3 Summary: Dictionary Attacks on Old Clicks and Field Clicks....................100

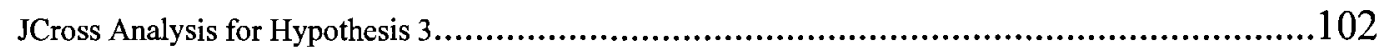

Hypothesis 3 Summary: JCross Patterns for New Clicks............................. 108

Hypothesis 3 Summary: JCross Patterns for Old Clicks and Field Clicks..................115

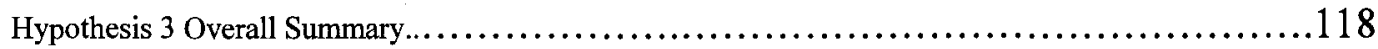

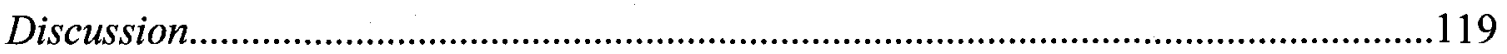

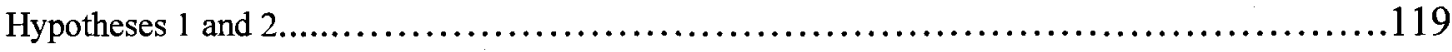

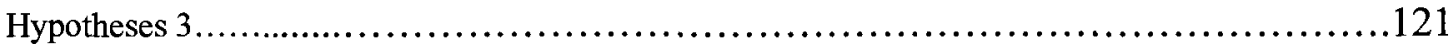

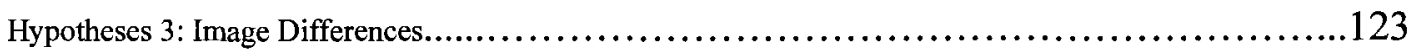

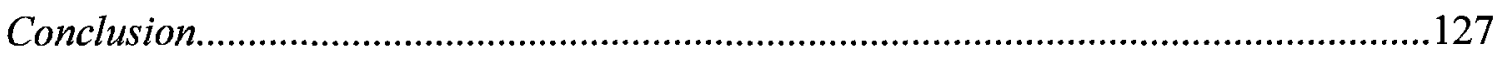

References Cited...........................................................................................131 


\section{List of Tables}

Table 1: Theoretical password space of graphical passwords.............................................8

Table 2: Theoretical password space of text-based passwords..............................................

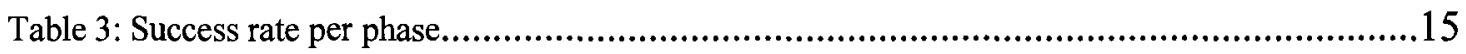

Table 4: Attempts per participant for each phase (field).........................................................15

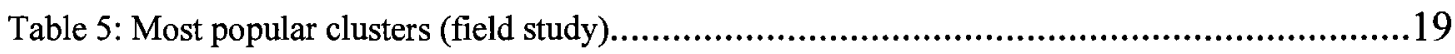

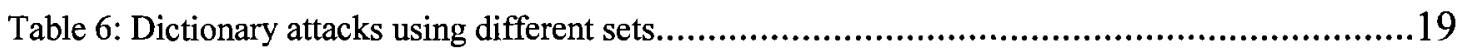

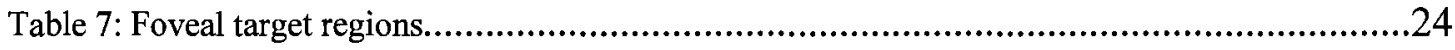

Table 8: Sample fixation data (FXD) file from the Tobii eye tracker.....................................32

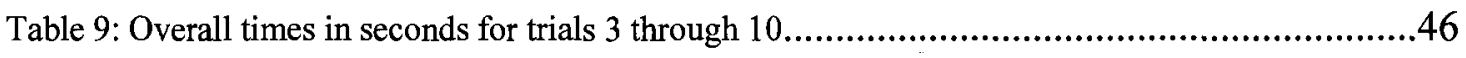

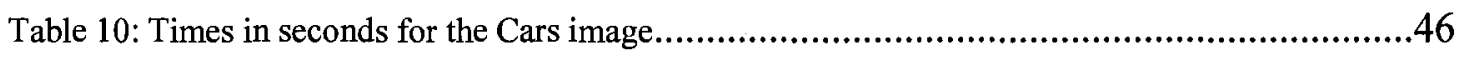

Table 11: Times in seconds for the Figureground image...................................................47

Table 12: Times in seconds for the Topdown image......................................................47

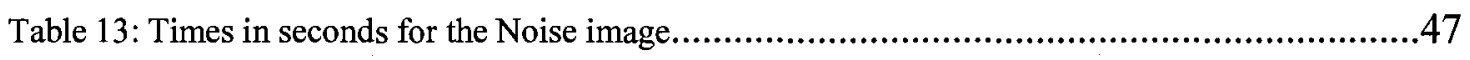

Table 14: Times in seconds for the Paperclips image.....................................................47

Table 15: Number of gaze fixations for both the PP and the IR conditions............................62

Table 16: Number of participants within each of the eight images........................................67

Table 17: Comparison of Analytical Results for the Heatmap Analysis....................................82

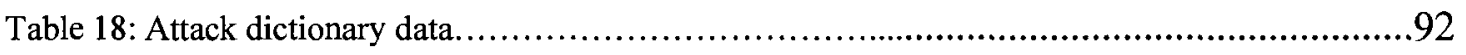

Table 19: Comparison of Analytical Results for the Dictionary Attack Analysis.........................101

Table 20: Attack dictionary data used in the analysis of variance........................................... 102

Table 21: The randomness of the JCross patterns for both PP and IR against New Clicks...............109

Table 22: The randomness of the JCross patterns for both PP and IR against Old Clicks.................116

Table 23: Comparison of Analytical Results for the JCross Analysis......................................117

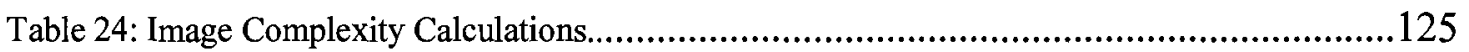




\section{List of Figures}

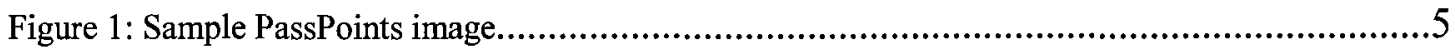

Figure 2: The Cars and Pool images........................................................................ 14

Figure 3: Median click-times for all participants per phase...................................................15

Figure 4: Median total times for all participants per phase...................................................15

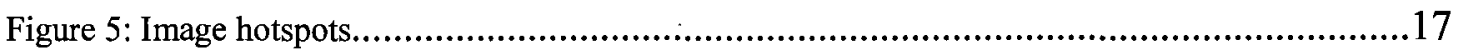

Figure 6: Clusters of popular click-points based on participant data on all 17 images.....................18

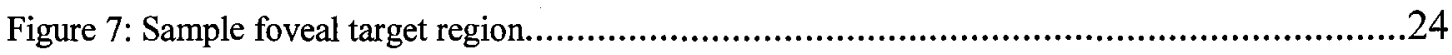

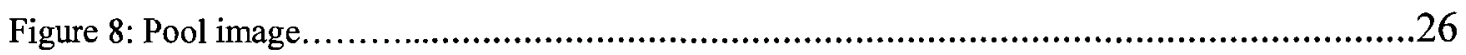

Figure 9: Pool image and click-points density map........................................................26

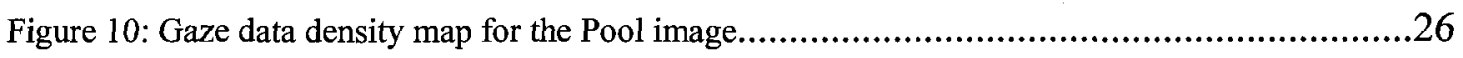

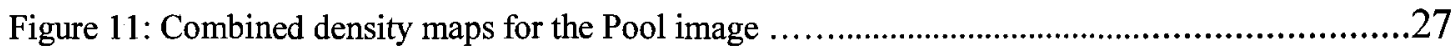

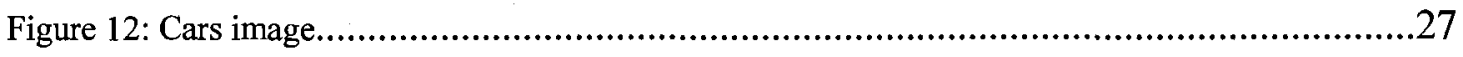

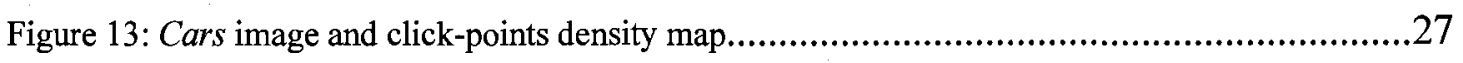

Figure 14: Gaze data density map for the Cars image.....................................................28

Figure 15: Combined density maps for the Cars image.....................................................28

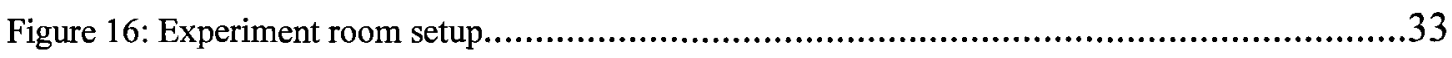

Figure 17: The Circus image and the Tribalwall image........................................................34

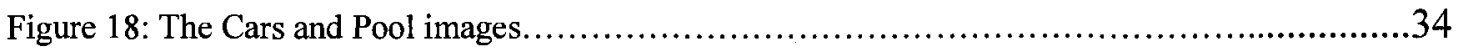

Figure 19: The Truck, Paperclips, and Statue images.........................................................35

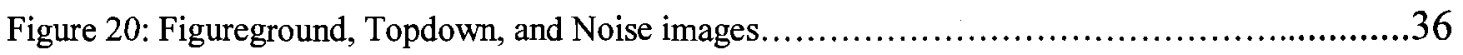

Figure 21: Create phase completion times.............................................................49

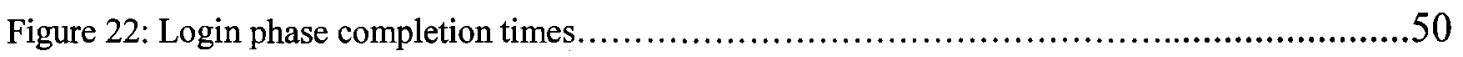

Figure 23: The gaze time ratios on the $\mathrm{X}$ axis for all gaze in the PassPoints condition.....................52

Figure 24: The gaze time ratios on the $\mathrm{Y}$ axis for all gaze in the PassPoints condition......................53

Figure 25: The gaze time ratios on the $\mathrm{X}$ axis for all gaze in the Image Recall condition...................55

Figure 26: The gaze time ratios on the $\mathrm{X}$ axis for all gaze in the Image Recall condition.................56

Figure 27: Gaze fixation durations for the PassPoints condition............................................59

Figure 28: Gaze fixation durations for the Image Recall condition.......................................60

Figure 29: Number of gaze fixations for the PassPoints condition......................................6

Figure 30: Number of gaze fixations for the Image Recall condition....................................64 


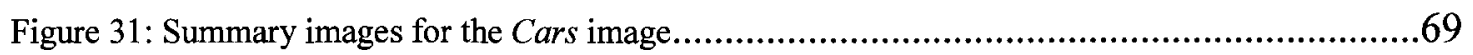

Figure 32: Summary images for the Pool image............................................................... 71

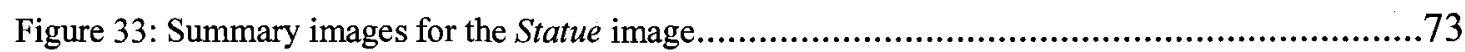

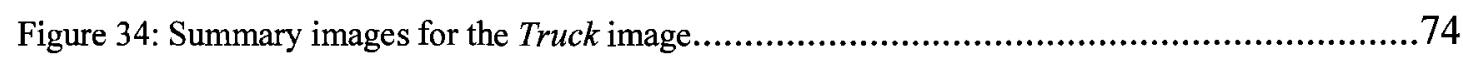

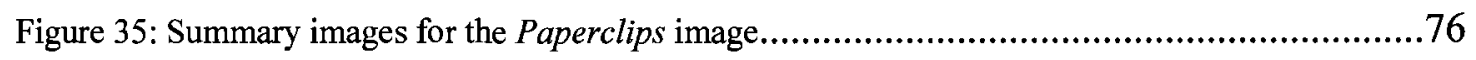

Figure 36: Summary images for the Figureground image..............................................

Figure 37: Summary images for the Topdown image.........................................................

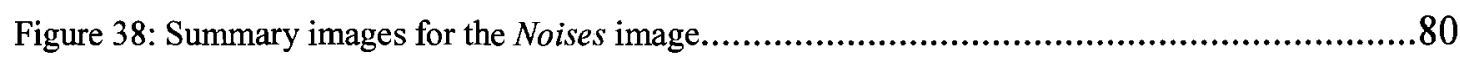

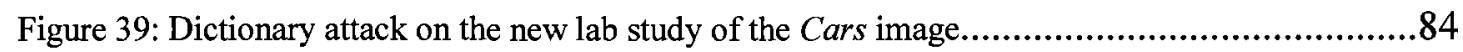

Figure 40: Dictionary attack on the new lab study of the Pool image......................................85

Figure 41: Dictionary attack on the new lab study of the Truck image........................................86

Figure 42: Dictionary attack on the new lab study of the Paperclips image...............................87

Figure 43: Dictionary attack on the new lab study of the Statue image......................................88

Figure 44: Dictionary attack on the new lab study of the Figureground image............................89

Figure 45: Dictionary attack on the new lab study of the Topdown image..................................90

Figure 46: Dictionary attack on the new lab study of the Noise image......................................91

Figure 47: Dictionary attack on the old lab study of the Cars image.........................................93

Figure 48: Dictionary attack on the old lab study of the Pool image.......................................94

Figure 49: Dictionary attack on the old lab study of the Truck image........................................95

Figure 50: Dictionary attack on the old lab study of the Paperclips image...............................96

Figure 51: Dictionary attack on the old lab study of the Statue image........................................97

Figure 52: Dictionary attack on the field study of the Cars image..............................................98

Figure 53: Dictionary attack on the field study of the Pool image..............................................99

Figure 54: JCross graphs of the Cars image (new clicks).................................................. 104

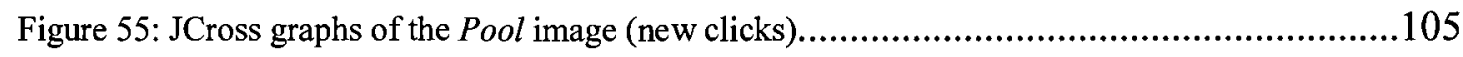

Figure 56: JCross graphs of the Truck image (new clicks)..............................................105

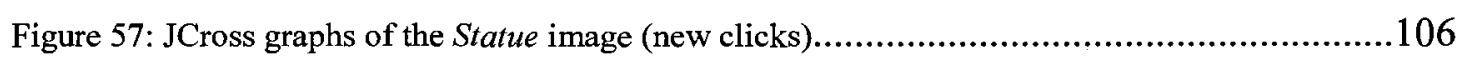

Figure 58: JCross graphs of the Paperclips image (new clicks).............................................106

Figure 59: JCross graphs of the Figureground image (new clicks).........................................107

Figure 60: JCross graphs on the Topdown image (new clicks)..........................................107

Figure 61: JCross graphs of the Noise image (new clicks)................................................ 108 
Figure 62: The randomness of the JCross patterns for both PP and IR (new clicks)..................... 109

Figure 63: JCross graphs of the Truck image (old clicks).....................................................11

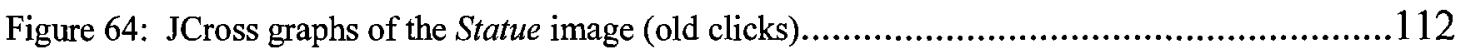

Figure 65: JCross graphs of the Paperclips image (old clicks)................................................113

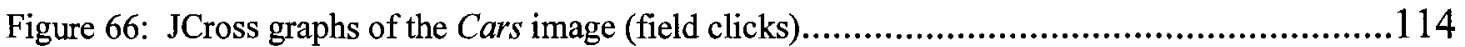

Figure 67: JCross graphs of the Pool image (field clicks)...........................................115

Figure 68: The randomness of the JCross patterns for both PP and IR (old clicks).....................117

Figure 69: Image Complexity Calculations................................................................ 125 


\section{List of Appendices}

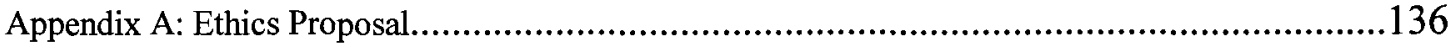

Appendix B: Announcement for Recruitment - Usability Test...........................................142

Appendix C: Consent Form - Usability Test - Graphical Passwords........................................143

Appendix C: Consent Form - Usability Test - Graphical Passwords - July 1st............................145

Appendix C: Consent Form - Usability Test - Graphical Passwords - SONA..............................147

Appendix C: Consent Form - Usability Test - Graphical Passwords - SONA - July 1st.................149

Appendix D: Consent Form - Usability Test - Image Memory Test.......................................151

Appendix D: Consent Form - Usability Test - Image Memory Test - July 1st............................153

Appendix D: Consent Form - Usability Test - Image Memory Test - SONA................................155

Appendix D: Consent Form - Usability Test - Image Memory Test - SONA - July 1st...................157

Appendix E: Participant Information - Usability Test - Student..........................................159

Appendix E: Participant Information - Usability Test - Non-Student......................................161

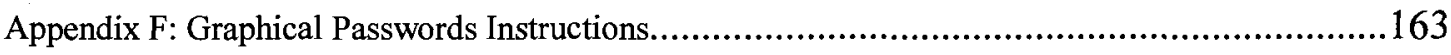

Appendix G: Image Memory Test Instructions........................................................164

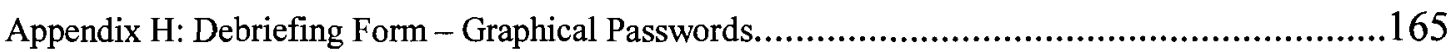

Appendix H: Debriefing Form - Graphical Passwords - July 1st......................................166

Appendix I: Debriefing Form - Image Memory Test....................................................167

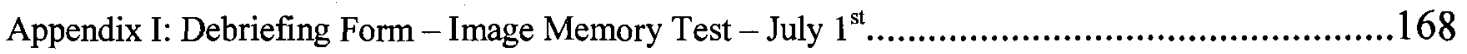

Appendix J: Post Task Questionnaire - Graphical Passwords..............................................169

Appendix K: Post Task Questionnaire - Image Memory Test................................................173

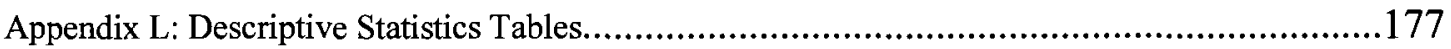

Appendix M: Clearview Software Fixation Algorithm.....................................................181

Appendix N: Detailed Explanation of the JCross Statistic..................................................182 


\section{Introduction}

With an increasingly large number of Internet websites being created for and frequented by a variety of users from both recreational and business worlds, the concepts of usability and security are being reassessed. The security involved with passwords is always of concern when dealing with websites that may contain information crucial to a person's own well-being, in terms of financial or other types of information. Also, memorability plays an important part in the usability of any password system. One idea that was introduced recently into the literature was the notion of graphical passwords (Blonder, 1996). Humans tend to have a better ability for remembering things that have graphical components (Gold, Murray, Sekuler, Bennett, \& Sekuler, 2005). Graphical passwords utilize images instead of text so they can take advantage of the longer decaying time of our visual memory than would be the case with text-based passwords. By offering users an alternative method by which to create their passwords, we can then assess the differences, advantages, and disadvantages of both types of password methods in terms of their usability and their level of security. This thesis addresses the security of graphical passwords.

In the field of security, there are three general authentication techniques which are currently being used as a means of accessing secured information (Menezes, Van Oorschot, \& Vanstone, 1996). These are:

1 - Something known: This refers to an authentication medium that utilizes remembered information allowing users access to their services, such as character-based passwords, graphical passwords, and PINs. 
2 - Something possessed: This normally refers to something tangible, which allows a user access to privileged information, such as a magnetic-striped bank card to identify itself to the receiving system (e.g. an automated transaction machine).

3 - Something inherent: This refers to making use of biometrics, or the collection of human characteristics, to identify the user attempting to access services. Examples of biometrics include fingerprints, voice-recognition, vascular structures, retinal patterns, and many others.

We argue that the first mentioned method of authentication, something you know, such as passwords, is still a very important method to use, for many reasons. Passwords are intangible and, as such, are not something that others can physically take like bank cards or magnetic pass-keys that allow access to secured areas. Although intangible, passwords do carry a number of risks: they can be guessed by other users, they can be seen when a user is entering their password, or they can be forgotten, leaving the user blocked from gaining access. Passwords allow users to protect their access to various resources or services, while maintaining their independence from one to the next; a user is also able to change the desired password as needed. Biometrics, based on something that is unique to every individual, on the other hand, could potentially lead to the compromising of privacy; this would be the case if a specific user's collection of accounts was directly linked to the identity of that user. As biometrics are unique to every individual, they are almost impossible to change. The simplicity and low cost of passwords, although not infallible, suggest some balance to the risk pertaining to their use. 


\section{Graphical Passwords}

For many years, people have been using text-based passwords to protect their accounts. It is becoming easier for attackers to create and use software that guesses users' account passwords; as such, this is quickly becoming a security issue. The term attacker refers to someone attempting to gain unauthorized access to a computer or electronic records. For example, it might refer to someone proficient in computer use who excels in finding users' information, and then uses it against them in order to obtain passwords to their protected accounts. Alternatively, an attacker can also look make use of a dictionary of common words in order to continuously guess the password for the account in question.

By inventing graphical passwords, Blonder (1996) offered an alternative form of protecting anyone's personal account from the threat of computer attackers. Blonder's style of graphical passwords allows only the use of pre-processed images; the click regions can only be chosen from specific pre-designed regions in each image. To create a password, a user must select which of the regions they will click in, and to login, they must click in those same regions. More recently, several other graphical password schemes have been proposed, as are described below. More comprehensive surveys are available in the literature (Suo, Zhu, and Owen, 2005; Monrose and Reiter, 2005).

The PassFaces method (Brostoff \& Sasse, 2000) involves the user making a choice between a wide selection of people's faces in order to create their password. Passfaces works as follows: users select one face from an array of nine faces, repeating this step three more times for three more arrays of nine faces. In order to login, the user in question must then reselect the correct face from each of the four arrays of nine faces. 
The presenting of the arrays of faces occurs one at a time, and the photographs in the array remain constant; each face does not repeat itself within or between arrays. However, the location of each face within each array of faces is randomly distributed every time it is presented to the user. This feature helps secure a user's Passfaces password from detection through observation (known as shoulder-surfing).

Much like the Passfaces authentication scheme, Déjà vu (Dhamija \& Perrig, 2000) makes the user select from arrays of images, but uses randomly generated abstract color images from a database of thousands of images to create a password portfolio. This password portfolio, comprised of 5 images selected by the user, is then linked to the unique username of that person. When attempting to login at a later time, the user enters his or her username, upon which the Déjà vu system generates and displays 25 of these abstract color images, 5 of which are the user's selected images comprising the password in question. The user must then correctly identify which 5 of the 25 images comprise the password. This password system has some potential advantages, such as reducing the likelihood that users will select weak passwords, and it also makes it difficult to write down or share their passwords with other users.

Another method of utilizing graphical passwords was developed by Jermyn, Mayer, Monrose, Reiter, \& Rubin (1999) who proposed a novel idea where users would draw an image they believed would be unique to them, all the while being easy enough to remember; this graphical password notion was developed and thereafter named the DrawA-Secret (DAS) scheme. The idea of DAS was developed in order to offer users a language-free method of password creation; since this scheme is composed of a picture drawn on a simple grid, users may find this easier to remember and attackers may find it 
more difficult to guess. (However, analysis by Thorpe \& van Oorschot (2007) suggests otherwise.)

In this thesis, we will focus on the click-based graphical password scheme PassPoints that extends the suggestion of Blonder (Birget, 2006). These graphical passwords work much the same way as text-based passwords, in that they are created using a series of individual locations (i.e. click-points instead of keystrokes), selected in a specific order at different locations on a 2-dimensional image. For example, as we can see from Figure 1, the user in question has clicked on five different locations on one image, in a specific order, thus creating a unique graphical password.

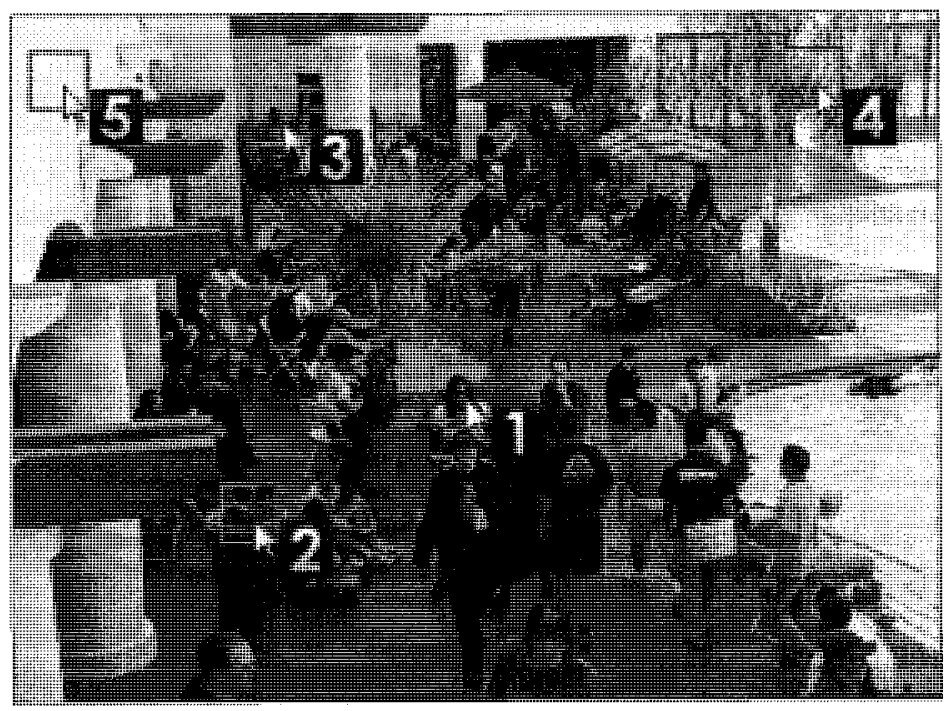

Figure 1. Sample PassPoints image. In this particular image, the numbered squares show where the users clicked, in order of click-points, to create his or her graphical password. The orange squares represent the tolerance region, of 19 by 19 pixels surrounding the original click-point.

Advantages of graphical passwords over text-based passwords

Researchers make several arguments about why graphical passwords are better than text-based passwords. Firstly, they suggest that the memorability of graphical 
passwords may be superior, given the fact that people have very good memory for visual stimuli such as images and visual scenes (Wolfe, Horowitz, \& Michod, 2007). From this knowledge, we can surmise that users will be better able to remember graphical passwords over text-based passwords, given their capacity for remembering images that comprise objects and visual scenery. Graphical passwords will take advantage of visual cues to guide users' recall of graphical passwords, while text-based passwords do not share this same distinct advantage due to the fact that the text-box used for entering characters does not provide any memory cueing.

Another argument involves the potentially higher level of complexity possible in using images over text to create passwords. This relates to the threat model of exhaustive search. In this model, attackers systematically check the theoretical password space in order to gain access to users' accounts. The theoretical password space is every possible combination of letters, numbers, and characters that could potentially be used to comprise a password. An example of this would be to take a look at the possible combinations of a bank account holder's PIN (Personal Identification Number). If a user is required to enter a 4-digit password, selecting only numbers from zero to nine, then an attacker simply has to exhaust every possible combination between 0000 and 9999 in order to gain access to that particular user's account. This threat model raises serious security concerns relating to the use of typical text-based passwords.

Currently, the PassPoints systems that have been tested don't take full advantage of the theoretical password space that is available. The theoretical password space is potentially much larger for graphical passwords than it is for text-based passwords. To illustrate this, we compare two theoretical spaces for PassPoints, the 451 by 331 image 
resolution that has been tested and a 1024 by 768 image resolution, against the most widely used text-based password scheme, the 95 character password system (see Table 1 and Table 2 below). Most user login password systems today ask users to create textbased passwords that are approximately eight characters long; if we take an eight character password containing a string of eight characters ranging from $A$ to $Z$, a to $\mathrm{Z}$, which can include all numbers and special characters, we can easily calculate the theoretical password space as $95^{8} \approx 5.4 \times 10^{23} \approx 2^{53}$.

When we come to calculate the theoretical space in graphical passwords, we can first use our example of creating a graphical password of only five clicks per image. In the proposed PassPoints scheme (Birget, 2006), replicated for study by our colleagues (Chiasson, Biddle, \& van Oorschot, 2007), we know that the image resolution is 451 pixels in width by 331 pixels in height. As we know that each click encompasses a tolerance area of nineteen by nineteen pixels, we can fit approximately 414 distinct clicks for each graphical password in a single image; this means that the theoretical password space for five clicks per image is $414^{5} \approx 1.2 \times 10^{13} \approx 2^{43}$.

Next, we take as an example the theoretical password space of graphical passwords, using an image resolution of 800 by 600 , as well as an eight click password combination. Even though this has yet to be studied by researchers, it is fully within our ability to test experimentally. Using this resolution, a user could pick from approximately 1330 distinct clicks, which would result in a theoretical password space of $1330^{8} \approx 9.8 \mathrm{x}$ $10^{24} \approx 2^{83}$. We can see by comparing the latter theoretical space of graphical passwords against the most widely used 95 bit text-based password system, graphical passwords 
offer a potentially much larger theoretical password space than text-based passwords.

These and other examples are represented in Table 1 and Table 2 below.

Table 1. Theoretical password space of graphical passwords.

\begin{tabular}{llllll}
\hline $\begin{array}{l}\text { Tolerance } \\
\text { (pixels) }\end{array}$ & $\begin{array}{l}\text { \# Clicks } \\
\text { required }\end{array}$ & $\begin{array}{l}\text { Image } \\
\text { Resolution } \\
\text { (pixels) }\end{array}$ & $\begin{array}{l}\text { \# Clickable } \\
\text { points }\end{array}$ & $\begin{array}{l}\text { Theoretical } \\
\text { password space }\end{array}$ & Bits \\
\hline $19 * 19$ & 5 & $451 * 331$ & 414 & $1.2 * 10^{13}$ & 43 \\
$19 * 19$ & 8 & $451 * 451$ & 414 & $8.6^{*} 10^{20}$ & 70 \\
$19 * 19$ & 5 & $800 * 600$ & 1330 & $4.2^{*} 10^{15}$ & 52 \\
$19 * 19$ & 8 & $800 * 600$ & 1330 & $9.8 * 10^{24}$ & 83 \\
$13^{*} 13$ & 5 & $800 * 600$ & 2840 & $1.8^{*} 10^{17}$ & 57 \\
$13 * 13$ & 8 & $800 * 600$ & 2840 & $4.2^{*} 10^{27}$ & 92 \\
$13 * 13$ & 5 & $1024 * 768$ & 4653 & $2.2^{*} 10^{18}$ & 61 \\
$13 * 13$ & 8 & $1024 * 768$ & 4653 & $2.2^{*} 10^{29}$ & 97 \\
\hline
\end{tabular}

Table 2. Theoretical password space of text-based passwords.

\begin{tabular}{lllll}
\hline Characters & Characters allowed & $\begin{array}{l}\text { \# Characters } \\
\text { in password }\end{array}$ & $\begin{array}{l}\text { Theoretical } \\
\text { password space }\end{array}$ & Bits \\
\hline 26 & Alphabet & 6 & $3.0^{*} 10^{8}$ & 28 \\
52 & Alphabet/Case sensitive & 6 & $2.0^{*} 10^{9}$ & 34 \\
52 & Alphabet/Case sensitive & 8 & $5.3 * 10^{12}$ & 46 \\
95 & Alphabet/Case & 8 & $5.4^{*} 10^{23}$ & 53 \\
\hline
\end{tabular}

Another important consideration when assessing either type of password is the effective password space of the password types. We previously dicussed the theoretical password space of text-based passwords, but users quite often use words, phrases, and numbers that have meaning for them when creating their passwords. When users opt to use these methods in the creation of their passwords, this greatly begins to reduce the password space actually used for unique passwords, the effective password space. For text passwords, the effective password space is what remains after the elimination of randomly generated characters, numbers and letters that could potentially make up a text- 
based password. When users create text-based passwords, they tend to use words or combinations of words (fluffy, 12april1976, ilovemycar) that are familiar to them (Adams, Sasse, \& Lunt, 1997). This kind of common password choice forms the effective password space of text-based passwords. If a user were to enter $21 \% 8 \mathrm{t} \$ g 87$ as their password, this would fall into the category of the theoretical, but not the effective, password space.

The effective password space raises a security concern. The dictionary attack threat model is based on using a fixed list of possible passwords; this list, comprising thousands to millions of candidate passwords, is used to attack users found within a given system. This process can be automated by using a password cracker such as John The Ripper (Openwall Project, 2008). This program allows attackers, upon gaining access to a password file for a specific online service, to compare a dictionary of passwords to each individual user's hashed password, in an effort to match two of the same, thus allowing the attacker to determine which password is allocated to a specific user's account.

Whether it is possible to mount dictionary attacks on a graphical password scheme such as PassPoints is the subject of our research. Defining the effective password space of graphical passwords is slightly more complicated than defining the effective password space of text-based passwords. Since we know the number of words in a dictionary, the number of months in a year, and the approximate number of persons' or pets' names, and so on, we can approximate the effective password space of text-based passwords. However, since graphical passwords are comprised of click-points on an image, it is more difficult to determine this password space. It seems likely that many users will click on similar areas of the image, based on salient characteristics of the 
objects and patterns found within the image in question, but this can vary from image to image, so there is no one direct measure of the effective password space of this type of password. We can never be quite sure how wide the range is of overlapping passwords which people create. Since they are not words, names, or dates, it is somewhat more difficult to understand and attack the effective password space. We discuss this issue in the next section of the thesis.

Attacks on text-based passwords and graphical passwords

One method of attack that can be effective against both text and graphical passwords is simply to trick users into revealing their passwords. Phishing websites are fake sites created in order to lure someone into submitting their personal information when they believe they are actually logging on to the real site, be it a bank website, an email website, or any other webside which allows public access. Once the phishing website has acquired the account holder's information, this is then used to login to the real account, where the information that was once private is no longer so; money can be stolen, credentials copied, and so forth. A similar approach might work for graphical passwords scheme such as PassPoints, as long as the attacker can present the right image.

Another method of obtaining users' passwords is through the use of keylogging software, or keylogging hardware, to record each keystroke performed by the user in question. Once the keystrokes have been recorded by the software or hardware, the attacker has access to each of the keys hit by the user, where he or she can then locate that user's passwords based on the information recorded by the program. Graphical passwords are much less open to attacks by very simple keylogging software or 
hardware, which record only keystrokes from the keyboard. However, more sophisticated (but clearly feasible) malware could record mouse clicks and screen images, and analyze them to obtain the correct sequences of click-points.

\section{Research Focus}

In this thesis, I will address the security of graphical passwords using an eye tracker. We will focus our attention on an implementation of the PassPoints system created by members of our Usable Security group (Chiasson et al., 2007). The eye tracker offers us a means of testing whether a person's gaze points, distributed over a wide range of users, would allow attackers to more accurately guess users' graphical passwords.

This thesis is divided into the following sections: this first section presents and discusses graphical passwords, their inception and their perceived value over text-based passwords, as well as introduces the main research question of this thesis; the second section discusses past relevant research in the literature dealing with graphical passwords, including the usability and security issues concerning graphical passwords, as well as some literature dealing with visual attention and visual search; the third section presents the hypotheses that will be tested in order to investigate the main research question, and introduces the method that will be used for the thesis research; and, the fourth section presents the results of the thesis research. We then provide some discussion of these results, and offer conclusions and suggestions for future research. 


\section{Main Research Question}

The main research question of this thesis is: can preselection gaze distribution statistics predict graphical password click-points? We will conduct a research study and compare the gaze data that we will gather with the click-points chosen by users as passwords. This could indicate that attackers would be able to guess where users click by simply observing where other users look on a given image. This is of particular interest when dealing with attackers who want to gain information on how to accurately guess users' passwords. Given the circumstance where an attacker would influence people to look at given images on a computer screen while he or she records their gaze data, he or she would then be able to analyze this data and create a database of likely click-points that users would use in order to create their graphical passwords. This would allow the attacker to more easily guess users passwords.

\section{Background}

In order to better understand why graphical passwords are a viable alternative to text-based passwords in everyday computer use, we must first examine two of their important aspects, the usability of graphical passwords and their security.

\section{The Usability of Graphical Passwords}

Wiedenbeck (2005) was the first to propose that testing the usability of the PassPoints graphical password system was an important part of discovering how users perceived and used graphical passwords. Her preliminary results showed that users created valid graphical passwords with relative ease over text-based passwords, but made 
more mistakes and took longer to login than did their counterparts. This seems to indicate that both graphical password and text-based password methods have their own problems in terms of usability and security issues, but also indicates that creating graphical passwords, which is obviously the newer method of the two, may have some distinct advantages over text-based passwords, and requires more in-depth research.

The study conducted by Chiasson, Biddle, \& van Oorschot (2007) looked at usability issues related to the creation and maintenance of click-based graphical passwords. They conducted both a small scale lab study with 43 participants followed by a larger field study. Their large-scale field study, which lasted for approximately seven to nine weeks, had roughly two hundred students participating in the experiment held during three university-level courses over the course of one semester. Roughly $95 \%$ of these two hundred participants opted to use graphical passwords when given the choice to use either a graphical password or a text-based password; this password was then required to allow them to $\log$ in to a specific website that was necessary for the course they were registered in. The remaining $5 \%$ of the participants chose to use a text-based password to $\log$ in to this same system for the duration of the semester. The data for these latter participants was not collected, since text-based passwords were not an integral part of the study. 
a) Cars Image

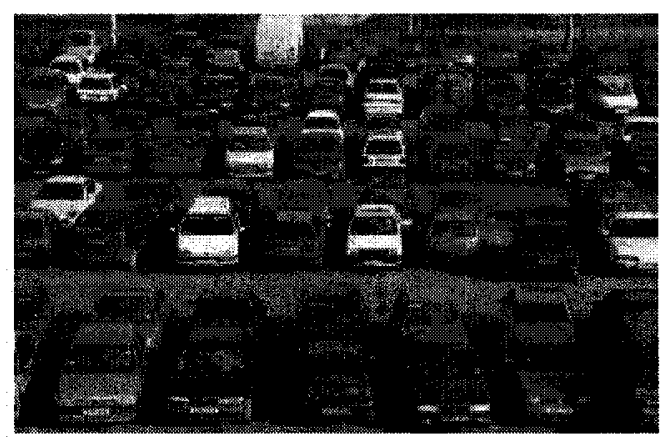

b) Pool Image

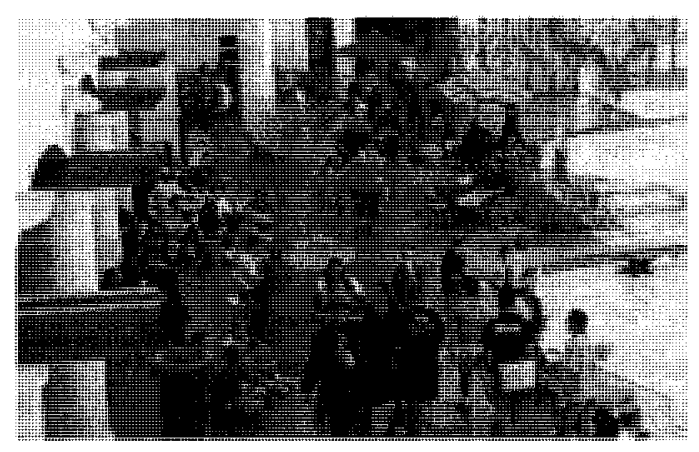

Figure 2. These two images were used in the old lab study: (A) the Cars image; and (B) the Pool image (Chiasson et al., 2007).

The goal of the study was to determine usability measures of an implementation of the PassPoints graphical password system, and offered some reliable information relating to the usability of each image and the process of using graphical passwords.

Figures 3 and 4 below demonstrate the times participants required to create, confirm, and login with their passwords using these two images; Table 3 shows how successful participants were at logging into the system using graphical passwords based on the two images outlined in the aforementioned figures; finally, Table 4 demonstrates the mean, median, and maximum number of attempts participants took to login to the system using their graphical passwords. 


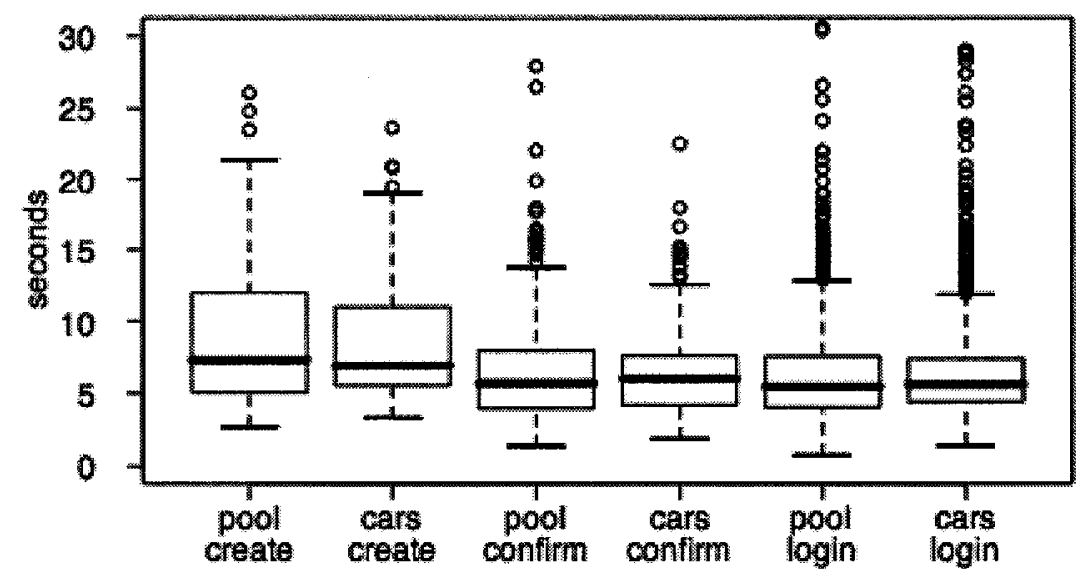

Figure 3. Median click-time for all participants per phase (Create/Confirm/Login phases) (Chiasson et al., 2007).

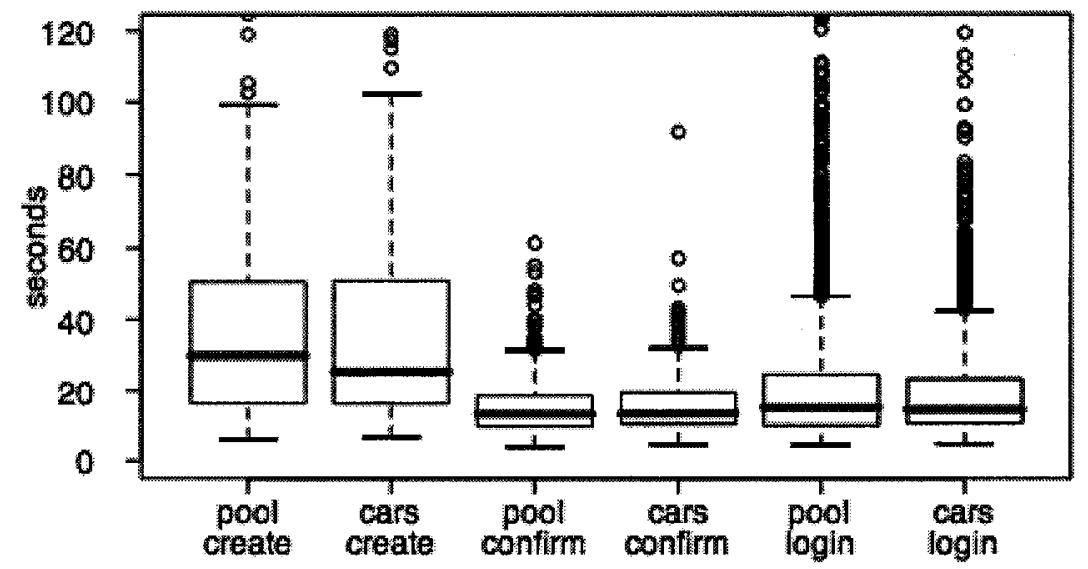

Figure 4. Median total times for all participants per phase (Create/Confirm/Login phases) (Chiasson et al., 2007).

Table 3. Success rate per phase (field) (Chiasson et al., 2007).

\begin{tabular}{l|cc}
\hline \multicolumn{1}{c|}{ Phase } & $\begin{array}{c}\text { Pool image } \\
\text { \# success / total } \\
\text { attempts }\end{array}$ & $\begin{array}{c}\text { Cars image } \\
\text { \# success / total attempts }\end{array}$ \\
\hline Confirm & $207 / 388(53 \%)$ & $170 / 293(58 \%)$ \\
Login & $1461 / 1880(78 \%)$ & $1301 / 1563(83 \%)$ \\
\hline
\end{tabular}

Table 4. Attempts per participant for each phase (field) (Chiasson et al., 2007).

\begin{tabular}{|c|c|c|c|}
\hline & Create phase & Confirm phase & Login phase \\
\hline Mean & 2.6 & 3.6 & 18 \\
\hline Median & 2 & 2 & 15 \\
\hline Maximum & 11 & 17 & 65 \\
\hline
\end{tabular}


Chiasson et al. (2007) interpret this data as indicating that participants performed satisfactorily in the field study at clicking on various points within each image in order to create a graphical password in each trial; this seems to indicate its potential for usability.

\section{The Security of Graphical Passwords}

A number of studies were recently conducted in order to address the security concerns related to graphical passwords. Dirik, Memon, \& Birget (2007) conducted the first analysis of the security of the PassPoints graphical password system. They developed a model intended to explore if it was possible to determine algorithmically whether a given image was suitable to be used in the graphical password scheme. They developed computer-generated attacks, based on the theories of visual attention, on the click-points data collected from participants in their study. This was done in an effort to predict the likely points on a given image where users would opt to select their clickpoints when creating their graphical passwords. Their results showed that their model had success in predicting user-selected click-points (Dirik et al., 2007).

The paper by Thorpe \& van Oorschot (2007) was based on research looking at the effects of identifying image hotspots (see Figure 5), which are defined as locations on an image where a large number of click-points are selected by users when creating their graphical passwords. It is important to note that the paper by Thorpe et al. (2007) and the previously mentioned paper by Chiasson et al. (2007) stemmed from the same research experiment; the data that was collected by these researchers was then interpreted differently in two separate areas. Every circle on each of these images demonstrates that participants clicked a number of times in the same area in order to create their passwords. 
The larger size of any specific circle illustrates that more users clicked on that same area of the image for their password. Each single spot indicates that only one participant selected to click on that particular area of the image when choosing a password.

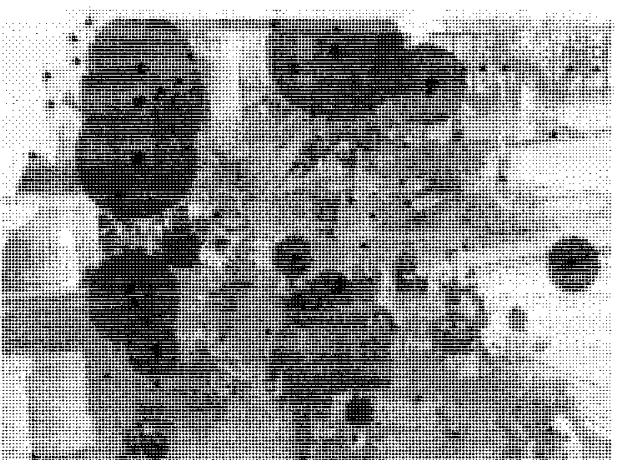

(a) pool

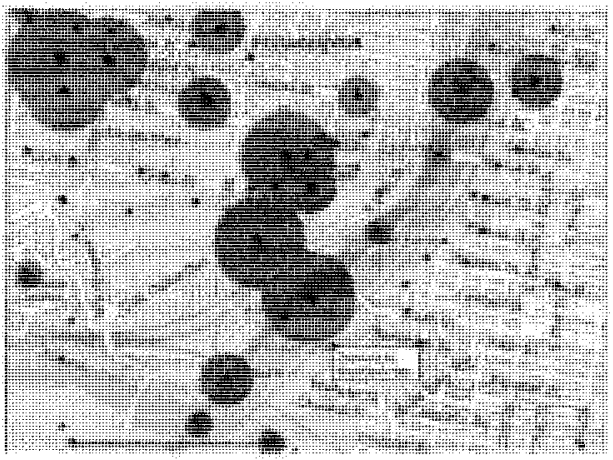

(c) phradelohis

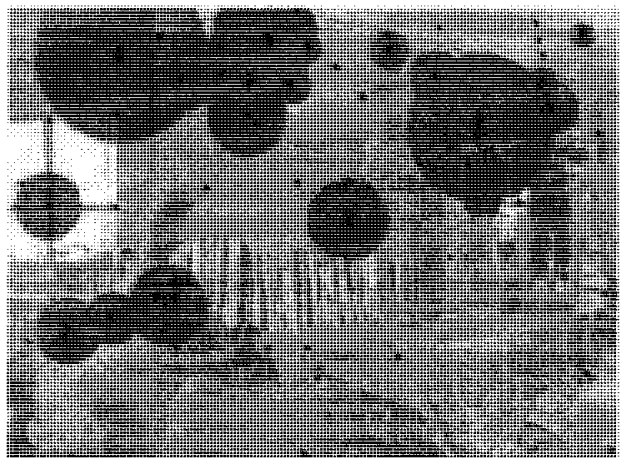

(b) wural

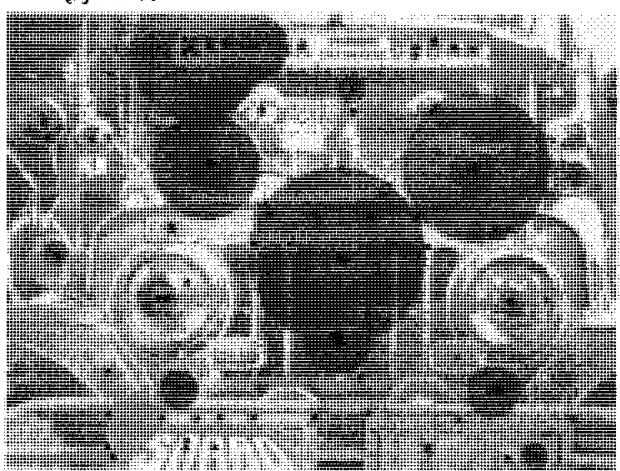

(d) theck

Figure 5. Image Hotspots. We can see the hotspots of these images from the click-points data from earlier research conducted by Thorpe et al. (2007).

The fact that one could relatively easily find hotspots located in each image offered these researchers the advantage of knowing where users tend to click most on specific images in creating their passwords; thus, human-seeded attacks would seem feasible on internet websites which utilize graphical passwords. This study entailed two data sets, the same as those studied in Chiasson et al. The first constituted the lab-controlled study where 43 users were asked to participate; the second experimental stage was the field test, lasting 
over a few weeks, and including over two hundred participants. The security research goal was to attempt to identify hotspots in the images used for the creation of graphical passwords, which were obtained by analysis of the participant click-point data in the lab study. This particular study made use of seventeen different images in the collecting of click-points data. Figure 6 below depicts the number of clusters of popular areas where participants clicked frequently.

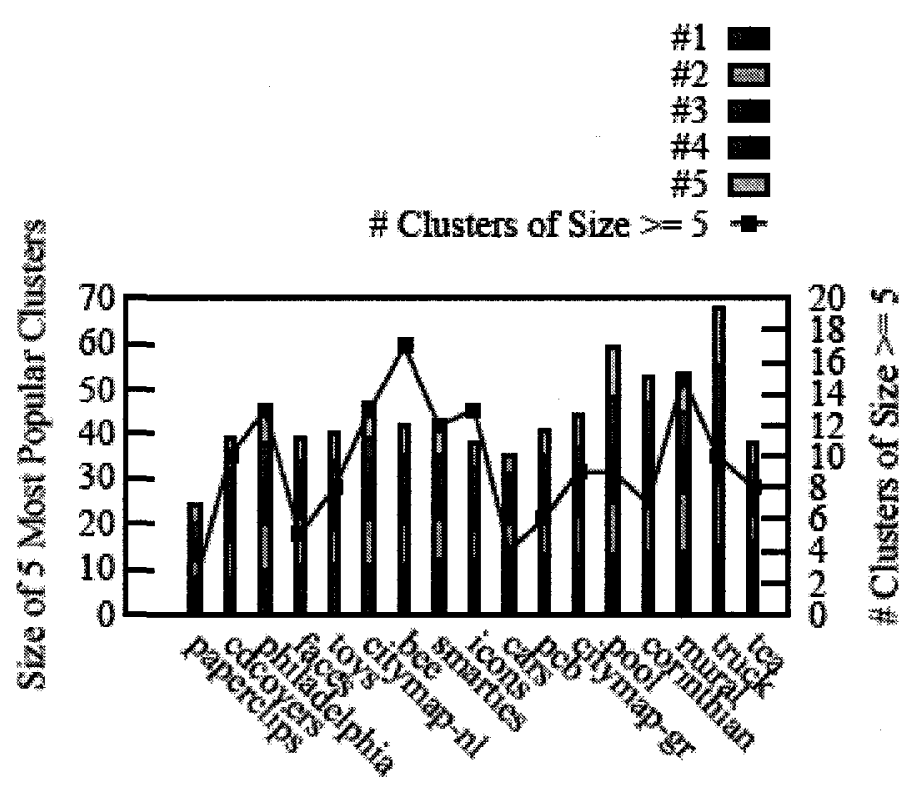

Figure 6. Clusters of popular click-points based on participant data on all 17 images (Thorpe et al., 2007).

Once the hotspots from these images had been identified, the researchers took their lab data for the Pool and Cars images, and made an attack dictionary in order to guess the field data passwords. They then carried out this human-seeded attack, which used participant data from the lab studies, as an attempt to guess the location of password click-points from the field study. Table 5 shows the sizes of the most popular hotspots for the field study, which included the Cars and Pool images. Table 6 shows the results from the dictionary attack. 
Table 5. Most popular clusters (field study) (Thorpe et al., 2007).

\begin{tabular}{l|cccccc}
\hline \multirow{2}{*}{ Image name } & \multicolumn{4}{|c}{ Size of most popular clusters } & \multicolumn{2}{c}{ \# clusters of } \\
& $\mathbf{\# 1}$ & $\mathbf{\# 2}$ & $\mathbf{\# 3}$ & $\mathbf{\# 4}$ & $\mathbf{\# 5}$ & size $\geq \mathbf{5}$ \\
\hline Cars & 26 & 25 & 24 & 22 & 22 & 32 \\
Pool & 35 & 30 & 30 & 27 & 27 & 28 \\
\hline
\end{tabular}

Table 6. Dictionary attacks using different sets. All subsets of users (after the first two rows) are the result of 10 randomly selected subsets of $u$ short-term study user passwords. For rows 1 and 2, note that $u=33$ and 35. $M$ is the alphabet size, which defines the dictionary bitsize. See Thorpe et al. (2007) for descriptions of $C^{V}$ and $C^{R}$. $†$ The first two rows use all data from the short-term study to seed a single dictionary, and as such, there are no average, max, or min values to report (Thorpe et al., 2007).

\begin{tabular}{|c|c|c|c|c|c|c|c|c|c|c|}
\hline \multirow[b]{2}{*}{ Set } & \multicolumn{5}{|c|}{$\begin{array}{l}\text { \# passwords guessed out of } \\
109\end{array}$} & \multirow[b]{2}{*}{$m$} & \multirow[b]{2}{*}{ bitsize } & \multicolumn{3}{|c|}{$\begin{array}{l}\text { \# passwords guessed out of } \\
114\end{array}$} \\
\hline & $m$ & bitsize & avg & $\min$ & $\max$ & & & avg & $\min$ & $\max$ \\
\hline$\overline{C_{\mathrm{u}}^{R}}$ & 165 & 36.7 & $37(34 \%)$ & 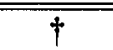 & $\bar{\dagger}$ & 175 & 37.1 & $59(52 \%)$ & $\dagger$ & $\overline{\dagger \dagger}$ \\
\hline$C_{\mathrm{u}}^{V}$ & 104 & 33.4 & $22(20 \%)$ & $\dagger$ & $\dagger$ & 77 & 31.1 & $41(36 \%)$ & $\dagger$ & $\dagger$ \\
\hline$C_{25}^{R_{25}}$ & 125 & 34.7 & $24(22 \%)$ & $9(8 \%)$ & $35(32 \%)$ & 125 & 34.7 & $42(37 \%)$ & $29(25 \%)$ & $56(49 \%)$ \\
\hline$C_{25}^{V}$ & 85 & 31.9 & $21(19 \%)$ & $7(6 \%)$ & $27(25 \%)$ & 59 & 29.2 & $34(29 \%)$ & $19(17 \%)$ & $47(41 \%)$ \\
\hline$C^{R}{ }_{20}$ & 100 & 33.1 & $22(20 \%)$ & $8(7 \%)$ & $32(29 \%)$ & 100 & 33.1 & $35(31 \%)$ & $24(21 \%)$ & $55(48 \%)$ \\
\hline$C_{20}^{V}$ & 72 & 30.6 & $17(16 \%)$ & $8(7 \%)$ & $30(28 \%)$ & 52 & 28.2 & $28(25 \%)$ & $18(16 \%)$ & $43(38 \%)$ \\
\hline$C^{R}{ }_{15}^{20}$ & 75 & 30.9 & $14(13 \%)$ & $4(4 \%)$ & $25(23 \%)$ & 75 & 30.9 & $30(27 \%)$ & $20(18 \%)$ & $45(39 \%)$ \\
\hline$C_{15}^{V}$ & 56 & 28.8 & $12(11 \%)$ & $4(4 \%)$ & $24(22 \%)$ & 41 & 26.4 & $26(23 \%)$ & $14(12 \%)$ & $43(38 \%)$ \\
\hline
\end{tabular}

It was hypothesized that by utilizing either of these methods, graphical passwords might be successful as targets for attackers to gain access to a user's accounts, based on the effective password space as discussed in the previous sections. They conclude that such attacks would indeed be practical. We now suggest an additional kind of human-seeded attack may be possible, that if attackers could easily gather data on the whereabouts of people's eye focus on graphical password images, this might also allow attackers to guess users' passwords.

\section{Visual Attention and Visual Search}

There has been little research in the use of eye tracking and computer security.

The main topic has been the use of eye trackers as input devices as an alternative to 
keyboards or keypads (De Luca, Weiss, \& Drewes, 2007; Kumar, Garfinkel, Boneh, \& Winograd, 2007). When it comes to text-based passwords, vision is not much of a critical factor, if at all, to the security of this password scheme. However, when dealing with passwords of a graphical nature, security issues relating to vision can become a real concern. This is due to the fact that humans tend to look at shapes, colors, and objects in visual space in a specific order; this is referred to as visual search (Hillstrom \& Logan, 1998). Visual search, which is a general theory of selective visual attention, is defined as a perceptual task requiring attention, where the person in question scans the visual environment for a particular object or feature (the target) among other objects or features (the distracters) (Muller \& Krummenacher, 2006).

We are in a better position today than ever before to understand the relationship between visual attention and visual scenes and images. As our research pertains to the use of gaze distribution data to predict graphical password hotspots, it would seem a logical step to consider the characteristics of objects and shapes within given visual scenes, or images. If we were to better understand the saliency of objects located within particular images or image segments, then we would be in a better position to make educated guesses as to where gaze hotspots occur in a given image. To do this, we must begin by analyzing the inherent complexity of visual images, and how people notice, and find, salient and non-salient characteristics in images as part of their visual search tasks in the selection of click-points to comprise their graphical passwords.

When people look at a particular visual scene, they are drawn to objects of either salient characteristics (Shao \& Brady, 2006), familiarity within the scene (Karacan \& Hayhoe, 2008), cueing of objects as part of attentional capture mechanisms (Theeuwes \& 
van der Burg, 2008), or self-motivated visual search mechanisms (i.e. top-down visual processing) (Folk, 2008).

Salient characteristics are one of the kinds of stimuli located within a given visual scene that can attract someone's attention. When we look at any given image, we tend to look for things that are interesting to us, which are salient, and that are not too obstructed by distractors. The attentional spotlight and spatial cueing are largely responsible for how we divide our visual attention among a myriad of available stimuli in our immediate environment. We can think of the attentional spotlight as a directional beam of light that reveals, and is limited to, what is within its path (Sperling, 1960). As such, humans can shift their attention from one location to another, but cannot absorb all of the information from both locations at one time. Spatial cueing in the environment allows us to divert our attention from one thing to another by simply suggesting to our visual system where our focus should be. Salient characteristics of objects in images, which grab visual attention, can be as follows: objects of varying outlines, such as corners, sharp or irregular edges; objects which are made up of bright, vibrant colors, as opposed to dark, bland colors; objects which appear to be in close proximity; and, spatial location motivating shapes such as arrows pointing to specific things within the visual environment (Chun \& Wolfe, 2000).

Familiarity of visual scenes represents another feature of visual attention. When a person is familiar with a given visual scene, attention is given to changes that occur to parts of the scene (Karacan et al., 2008). Karacan et al. (2008) found that people spend much more time looking at areas of a visual scene where something novel has appeared or something is unusual in the visual space. These researchers found that people are able 
to detect these changes in the environment even when their gaze is not specifically turned to the area where the novel object has appeared or a change has taken place in the visual scene. It is important to note that people are more rapidly able to detect any and all changes to the visual scene when these changes occur with higher levels of semantic information, such as consistency of objects, rather than when faced with inconsistent objects in the given visual scene.

Cueing also plays an important role in visual attention. As our study deals with presenting images to participants in a serial manner without prior instructions for locating any objects or patterns within the image, this aspect of visual attention is less relevant to our research objectives. However, cueing does play an integral part in better understanding visual attention; therefore, we present research literature to support its role in the search for objects in a visual scene.

Visual cueing is the presentation of specific stimuli to someone, whether it be done by another person or by a series of images, thus allowing the former to locate another stimulus relevant to the cueing object. As an example, if someone were to see a series of images, the first of which shows a small, red bird, this person would be more likely to easily find other red birds located within the subsequent images of visual scenes. Theeuwes et al. (2008) found that participants were highly likely to locate a target singleton (the cueing object) in a given image, after having been shown this image as the first visual stimulus. They also found, however, that after location of the cueing image, people's attention is drawn to highly salient areas of the given visual scene.

Top-down and bottom-up visual processing are other important parts of visual attention. With these processes, it becomes easier for us to locate things in visual space 
when we've become familiar with what we are looking at or searching for. In bottom-up visual processing, or exogenous attention, our attention is drawn by nearby salient objects, such as the flashing lights of an emergency vehicle, or the neon lights of a passing road sign; this process is done automatically and effortlessly.

In top-down visual searches, or endogenous attention, our attention is under our control, in that we decide where we want to look, and what we want to look for. In other words, it is said to be goal-driven, in that we guide our attention to locate a specific object within the environment. An example of top-down visual processing would be an attempt to locate a green object within a myriad of grey objects in a given visual field (Chun et al., 2000).

In visual search, quite often both bottom-up and top-down processing are used to some degree to allow for the best visual search possible. This is not only true for a specific environment in question, but can be broadened to other scenarios of a similar nature. If we use, as an example, a scenario that consists of a person looking for a red vehicle amongst a few different rows of orange cars, he or she will be able to locate the former object within a short amount of time. However, when the person in question does finally locate the object, then the process becomes much easier for the following trial, even if the vehicle changes location. This also holds true for any subsequent trials involving different scenarios with similar conditions (e.g. searching for a red shirt among rows of orange shirts): response time will drop as a direct result of exposure to similar experimental conditions (Koike \& Saiki, 2006).

Visual acuity is an integral aspect of visual search. This brings us to discuss the gaze area of the human eye, which is caused by the fovea of the eye. The fovea of the eye 
represents the focus point of vision, which is comprised of a circular area that receives visual stimuli with great accuracy. The foveal target is based on a continuously changing scale, based on the distance from the eye and the point of focus, but always measured on 2 degrees of arc (Duchowski, 2007). The recommended viewing distance away from a computer screen, according to the Government of Ontario (2004), is between 40 and 72 centimetres. Table 7 demonstrates a few different foveal target calculations based on different distances from the eye to the point of focus.

Table 7. Foveal target regions calculated using the following mathematical formula: $X=$ distance * $\operatorname{Tan}\left(1^{\circ}\right) * 2$.

\begin{tabular}{lll}
\hline Distance $(\mathrm{cm})$ & Angle (degree) $\times 2$ & Foveal Target Region $(\mathrm{cm})$ \\
\hline 40 & 1 & 1.3964 \\
50 & 1 & 1.7455 \\
55 & 1 & 1.9200 \\
60 & 1 & 2.0946 \\
70 & 1 & 2.4437 \\
\hline
\end{tabular}

Figure 7 demonstrates an example of the foveal target based on the average of the calculations found in Table 7.

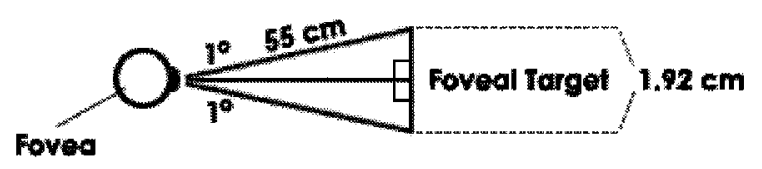

Figure 7. Foveal target region.

Studies of visual search show commonalities between participants. All of the principles of visual search will apply. Example activities are reading and driving (Rayner, 1995 and Land \& Lee, 1994). If this is true in graphical passwords, this may be a 
weakness exploitable by attackers. We suggest that gaze points gathered from any user could potentially be used in order to form an attack dictionary to guess another user's graphical password click-points.

\section{Preliminary Work}

A small scale preliminary research study was conducted, before the thesis study, stemming from the data from two aforementioned experimental lab studies (Chiasson et al., 2007) (Thorpe et al., 2007), in an effort to explore evidence for the risk users face when using graphical passwords as their method of choice to protect their information.

Even though the earlier research dealt with participants tested on a random sample of seventeen images, two of these images were of primary interest for our preliminary study: the Cars and Pool images. As these were the only images used in the earlier largescale field study of Chiasson et al. (2007), they allowed the best comparison with the gaze data from these two same images from our preliminary study. We then gathered gaze data from people looking at these images using an eye tracker.

A number of different visualizations were created in order to be able to interpret the similarities that exist between the click-points data and the gaze data from the lab studies. We created hotspot maps that show the density of either click-points or gaze points, coded by color, with green as the least dense areas, red as the middle density, and yellow as the highest level of density of either click-points or gaze points. 


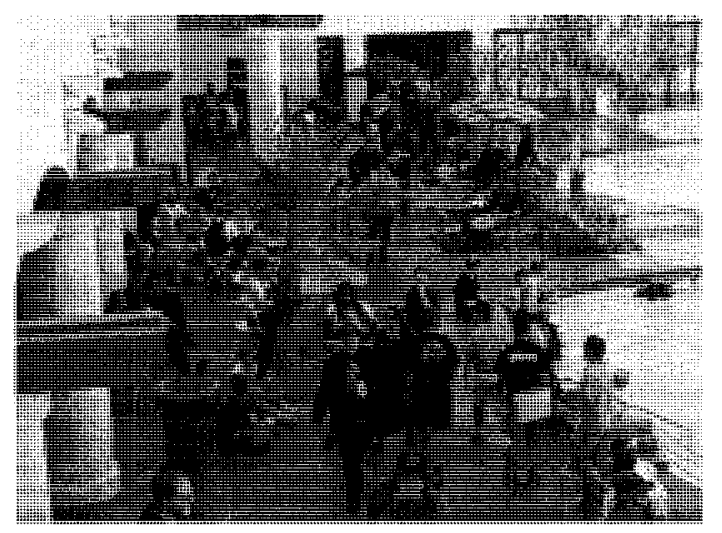

Figure 8. Pool image. This is the actual image that was used in the lab and field studies.

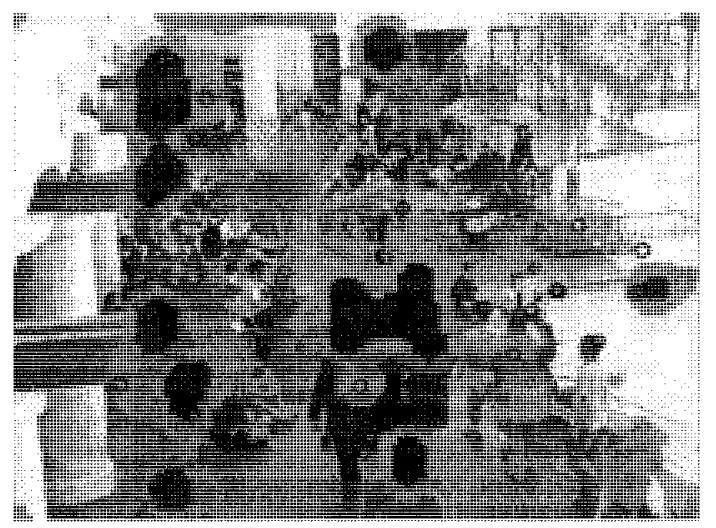

Figure 9. Pool image and click-points density map; the circles show the position of clicks from the preliminary lab study, while the density heat map is from the lab study from Chiasson et al. (2007).

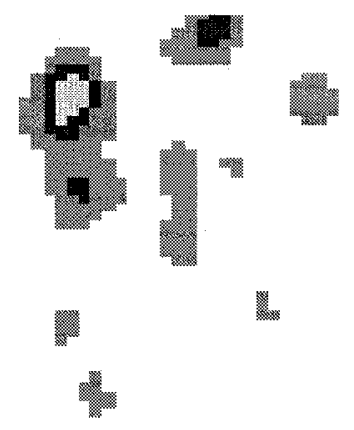

Figure 10. Gaze data density map for the Pool image. 


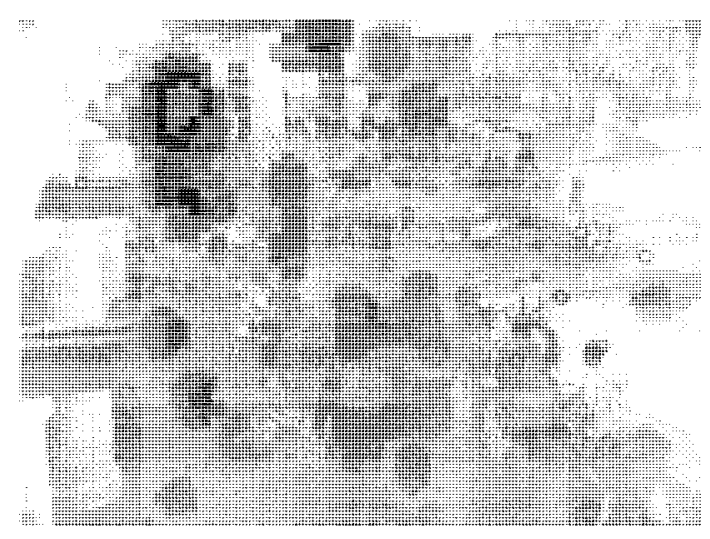

Figure 11. Combined density maps, with the gaze data overlapping the field click data for the Pool image.

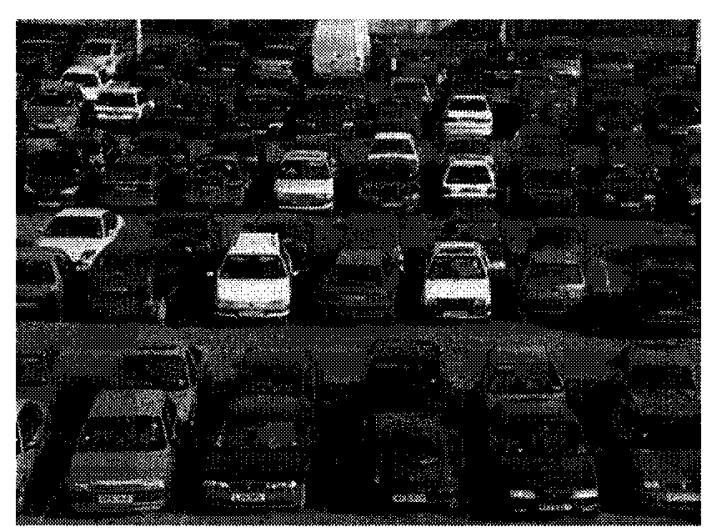

Figure 12. Cars image. This is the actual image that was used in the lab and field studies.

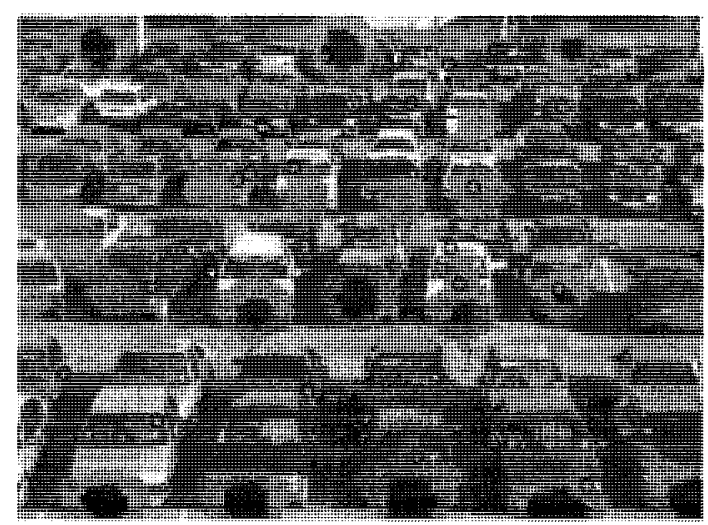

Figure 13. Cars image and click-points density map; the circles show the position of clicks from the prliminary lab study, while the density heat map is from the lab study from Chiasson et al. (2007). 


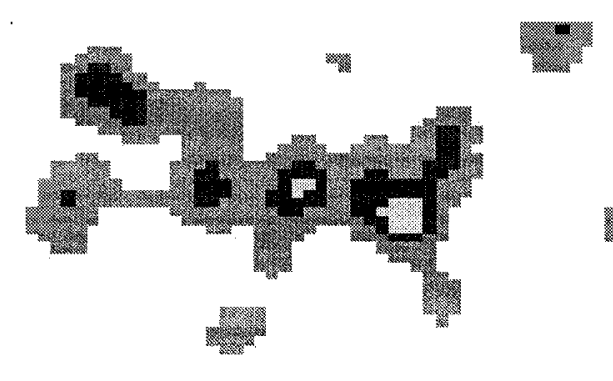

Figure 14. Gaze data density map for the Cars image.

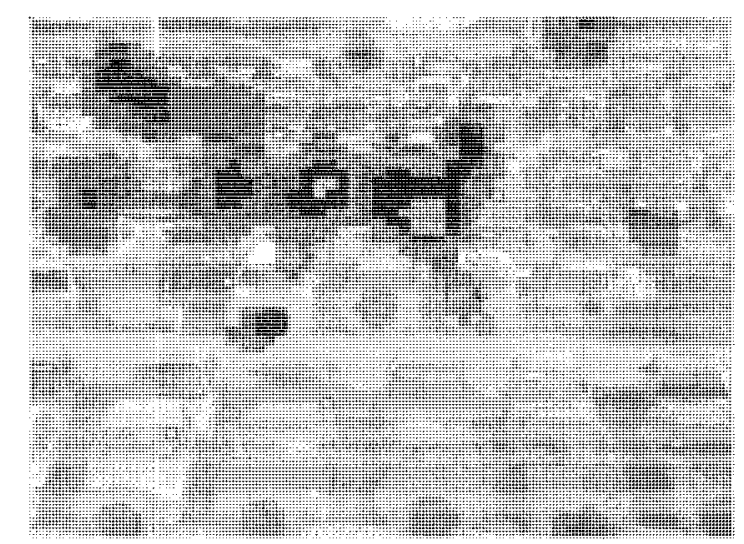

Figure 15. Combined density maps, with the gaze data overlapping the field click data for the Cars image.

Each of the density click-point or gaze-point images was created using the $\mathrm{R}$ statistical software program, including the spatstat package (Baddeley \& Turner, 2005). The images were created using the density function, which shows the relative intensity of points in an area. Figures 8 and 12 represent the actual images used in the experimental testing (i.e. the Pool and Cars images). Figures 9 and 13 depict the click-points density maps for each data set overlapping each image; these click-points density maps were achieved by calculating the density of various click-points hotspots using each of the corresponding data sets from the field studies of Chiasson et al. (2007). Figures 10 and 14 
demonstrate only the gaze data density maps for each of the data sets of the Pool and Cars images; these were achieved in much the same way as the click-points density maps, but were created using the gaze distribution data. Finally, Figures 11 and 15 depict the actual image (Pool or Cars) as the background image, overlapped by the click-points density map with a lower degree of opacity, as well as the gaze point density map with a higher degree of opacity; this was done in order to put more emphasis on the latter hotspot groupings.

Using only the naked eye in order to view the patterns located within these images, we can see that there is some degree of overlap between the gaze density patterns and the click-points density patterns; as these patterns overlap at specific areas of each image, it is apparent that a large number of users focused their attention on spots similar to those that other users had previously clicked. Having reviewed these images, we decided to investigate whether the regions where users click can be fairly well estimated looking solely at gaze data based on specific images.

\section{Research Study \\ Research Hypotheses}

In order to test our main research question, can preselection gaze distribution statistics predict graphical password click-points?, we first propose hypotheses as follows:

H1A: A left to right horizontal trend exists on the $X$ axis of the gaze pattern data.

H1B: A top to bottom vertical trend exists on the $\mathrm{Y}$ axis of the gaze pattern data. H2A: Fixation dwell-times decrease over the number of trials within subjects. 
H2B: The number of fixations decreases over the number of trials within subjects.

H3: Fixation point patterns resemble click patterns over the same picture across subjects.

\section{Method}

The experimental research study has two conditions, both of which require participants be invited to a specially fitted computer lab: the first condition (the PassPoints condition) required that participants create a total of ten graphical passwords, each comprised of five click-points on the image in question; the second condition (the Image Recall condition) required that participants look at the same ten images, but would not be asked to click on the images. While participants in either condition were busy performing their respective tasks, the system would record where they were looking on the screen through the use of the Tobii eye tracker. This would provide us with two means of collecting data for our analysis at the end of the experiment, one of which was gathering click-point data, the other one being gathering eye gaze fixation data via the eye tracker. By "gaze fixation data" we mean the location on the image in X-Y coordinates and duration in milliseconds of each fixation. The fixations will be separated by saccades, but it is the fixations themselves that will indicate cognitive engagement with a location on the image. Our lab study was designed to obtain and record three different sets of data, including click-points data from the PassPoints condition, and gaze fixation data from both the PassPoints and Image Recall conditions. 


\section{Click-points data}

For this lab study, a participant's click-point data refers to the five clicks required to make up one graphical password for each trial. In order to achieve this, we used an implementation of the PassPoints graphical password system, which gathered the clickpoints data for each participant. At first, this program showed each participant two trial images. It then showed the participant a random image from a selection of eight images, based on a latin square design. Click-point data was recorded and stored into a text file for each participant and for each trial image.

\section{Fixation data}

Fixation data originates from the gaze points which were recorded by the Tobii 1750 eye tracker (Tobii Technology AB, 2003), and then kept in a text file. The information of interest for this lab study was the data points that identify where the combined focus points of the left and the right eye were located on the image projected on the computer screen by the PassPoints program at a specific point in time (see Table 8 below), as well as the corresponding timestamp and duration during which these fixations occured. The timestamp allowed matching between click-point data and fixation data for analysis. The Tobii eye tracker Clearview software version 2.6.0 (Tobii Technology AB, 2006) collected gaze distribution data and calculated fixation data from it using the method of Dispersion Threshold (Salvucci \& Goldberg, 2000); we then used this fixation data in our analyses. Details of the Tobii algorithm can be found in Appendix M. We used the default settings of the eye tracker software, which were 30 pixels for the fixation radius, and 100 milliseconds as the minimum fixation duration. The timestamp column in 
Table 8 indicates at which point each fixation occurred, relative to the start of recording after calibration, in milliseconds. The duration column indicates how long, in milliseconds, each fixation lasted. The $\mathrm{X}$ and $\mathrm{Y}$ coordinates are the averaged coordinates of both eyes, measured in pixels, relative to the top-left of the physical screen.

Table 8. Sample Fixation Data (FXD) file from the EyeTracker.

\begin{tabular}{lllll}
\hline Fixation number & Timestamp (ms) & Duration (ms) & GazepointX (pxl) & GazepointY (pxl) \\
\hline 1 & 7 & 199 & 348 & 263 \\
2 & 226 & 239 & 395 & 299 \\
3 & 485 & 478 & 401 & 262 \\
4 & 984 & 718 & 388 & 296 \\
\hline
\end{tabular}

\section{Usability perception data}

Usability data was gathered in the form of a questionnaire given to the participants at the end of the sixty minute testing session, as well as by asking them to speak aloud their thoughts concerning what they were seeing on-screen at anytime during testing. This feedback was then recorded on the researcher's laptop for safekeeping until the final analysis could be done on the data from all participants in the study. We collected this data to ensure that the system worked as we intended, and the data does not specifically relate to our hypotheses.

\section{Equipment}

One Dell Pentium D personal computer, running Windows XP on a seventeen inch LCD monitor displaying an 800 by 600 pixel resolution, was used for each of the experimental sessions, along with an eye tracker built into an LCD monitor, two mice and two keyboards. The experimenter also had a laptop computer, which was used solely for 
note-taking based on observations, and the recording of comments using Microsoft Word and Windows Notepad.

The second of the LCD monitors used in this project contained the Tobii eye tracker. During each experimental session, this apparatus recorded individual gaze points for each participant's left and right eyes. The Clearview software running on the eye tracker hardware is what recorded and exported the gaze data for analysis at the last stage of this research project. Figure 16 demonstrates the setup of the eye tracker within the experiment room.

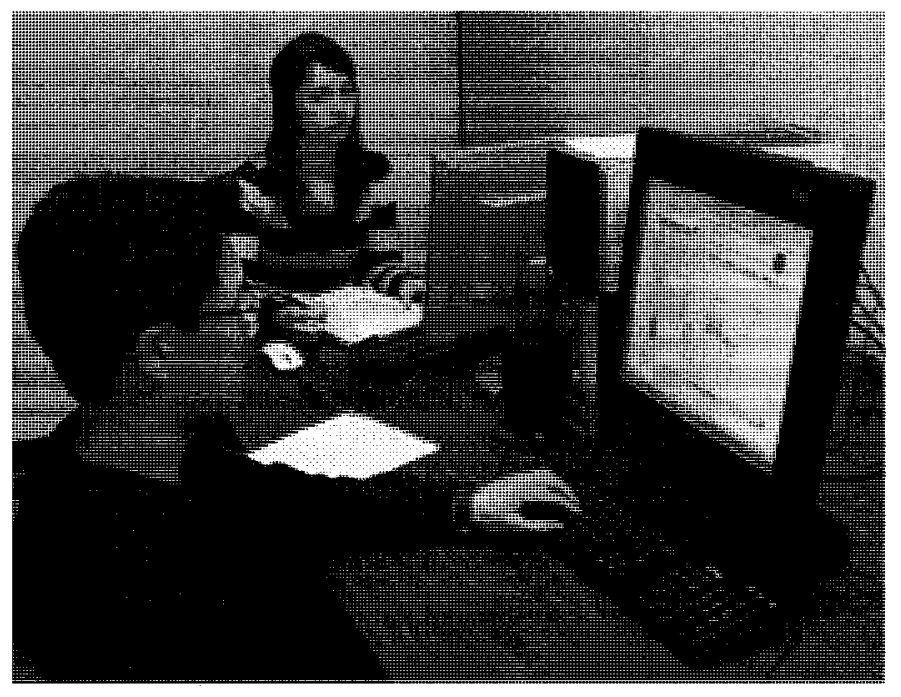

Figure 16. This figure represents the experiment room setup. The experimenter in the background sits away from the participant and makes notes on the participant's actions. The participant in the foreground is performing a task while the Tobii eye tracker records gaze data.

\section{Materials}

Our experimental conditions consisted of testing participants on a total of ten different graphical images. This section discusses which images we selected, and our motivation for opting to use these images in our testing methods. 

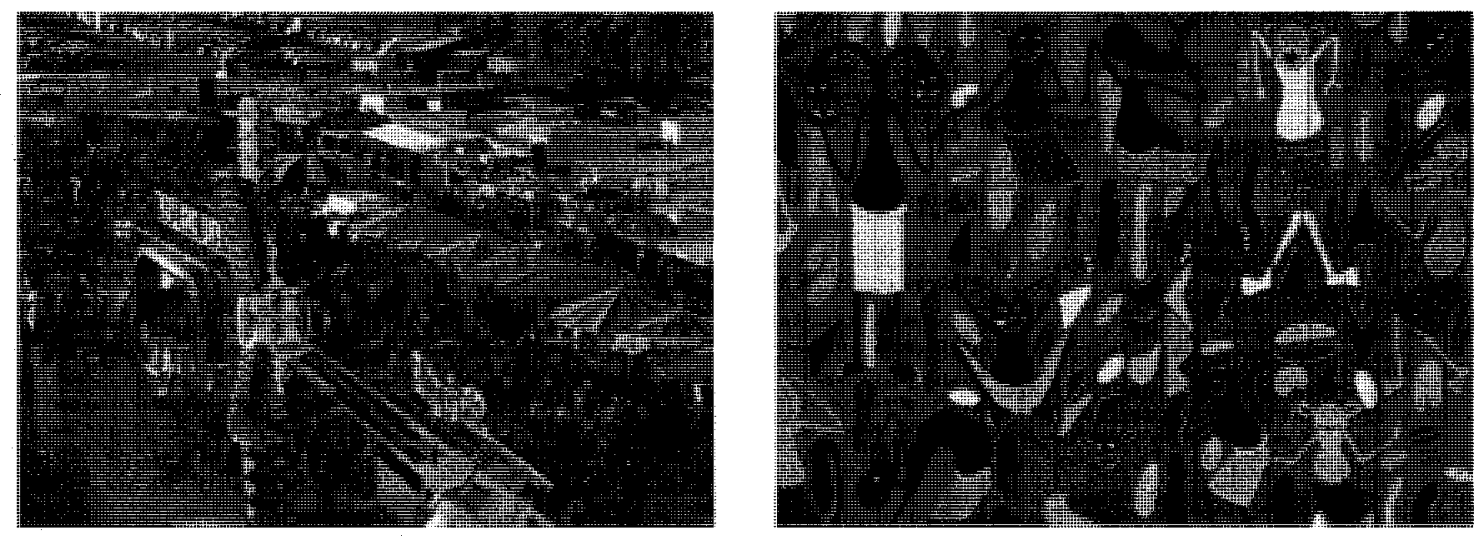

Figure 17. The two images used as practice trials in our study, for both the PassPoints and Image Recall conditions. The Circus image (left), and the Tribalwall image (right).

The first two images (Figure 17) that were used in our study were practice trial images.

Each participant was shown these two images, in succession, as a means of practicing and getting used to the task in either of the two conditions. We selected these two images, as they had previously been used for this same purpose in the study by Chiasson et al. (2007), and appeared to fit well when participants were introduced to their task.
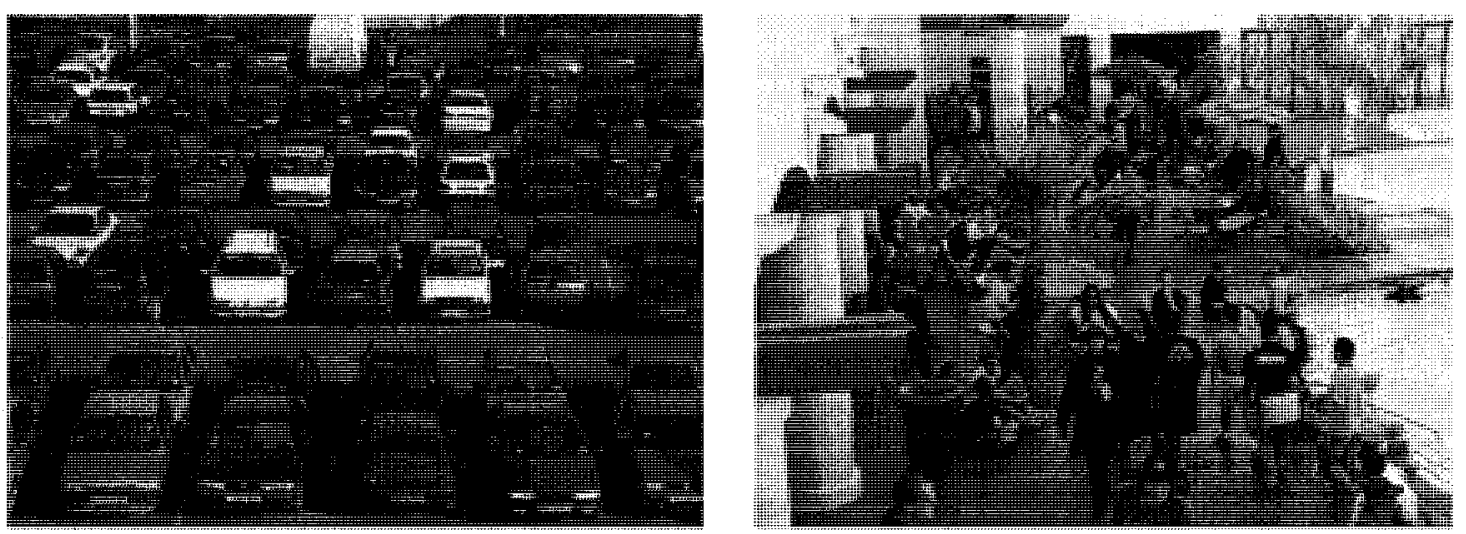

Figure 18. The images taken from a previous field study. The Cars image (left) and the Pool image (right).

The Cars and Pool images were used in our study for two very specific reasons: first, the images depicted in Figure 18 were part of an earlier field study conducted by Chiasson et 
al. (2007), which meant that there was a lot of click-point data available to us for further analysis with our own data; second, these images had previously been shown to provide users with a good user experience when given the task of selecting click-points in order to form a graphical password.
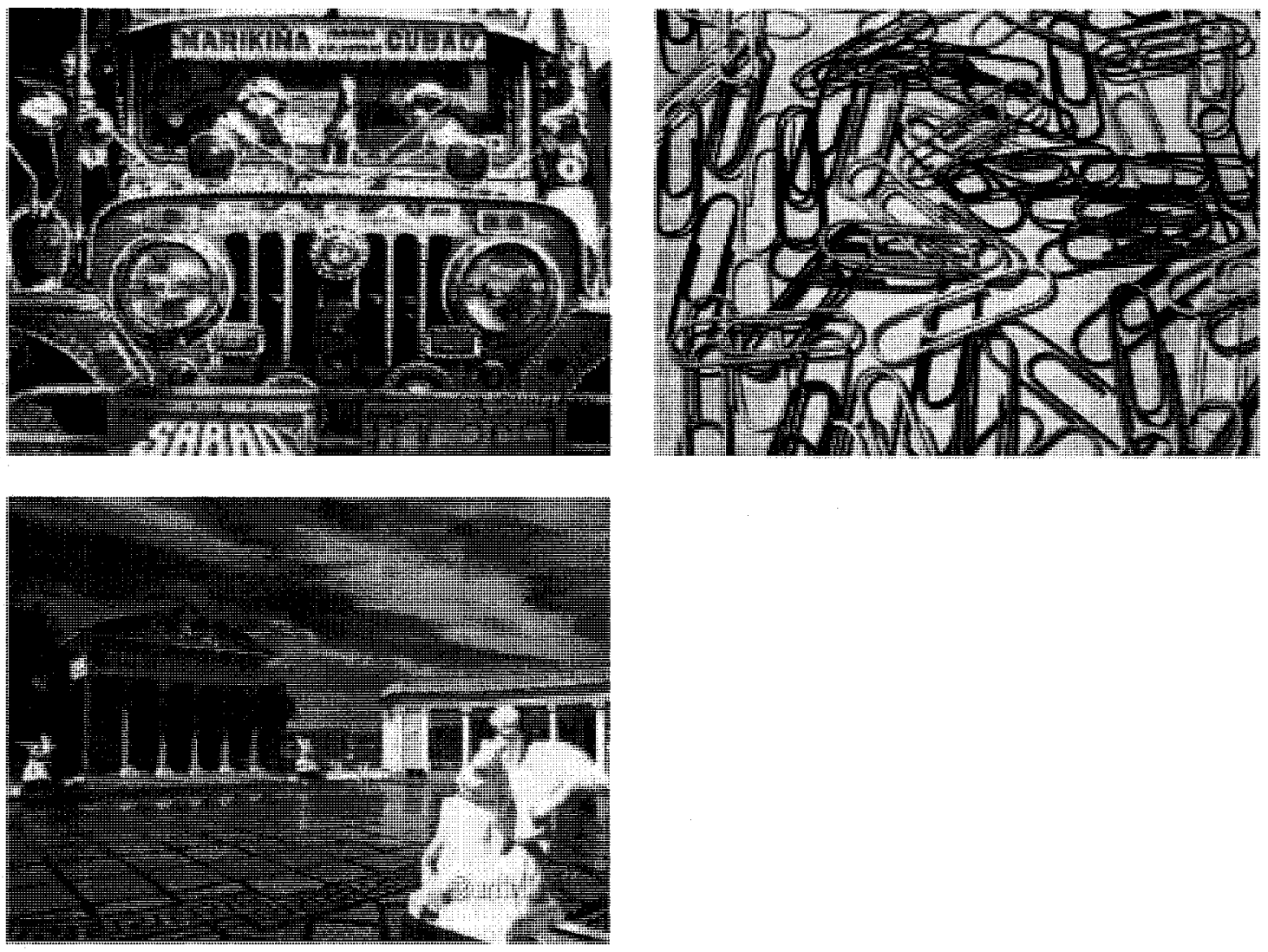

Figure 19. Images 5 through 7 used in our study. The Truck image (top-left), the Paperclips image (top-right), and the Statue image (bottom-left).

We selected the images found in Figure 19 from the collection of images from the

Chiasson et al. (2007) study, as we deemed them to be different enough that they would yield interesting results in terms of usability feedback data, click-points data, as well as eye gaze data from our participants in both experimental conditions. 

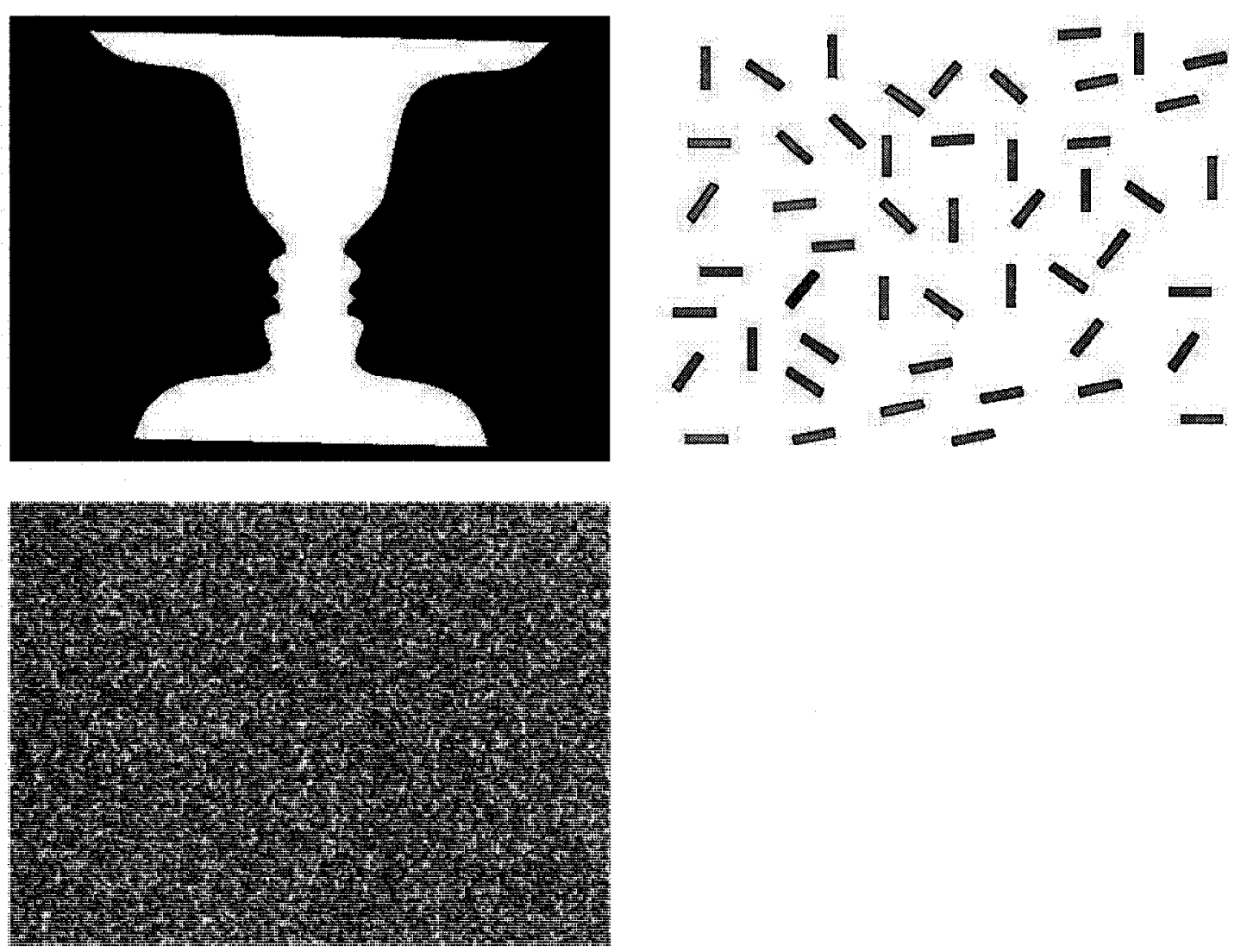

Figure 20. Images 8 through 10 used in our study. The Figureground image (top-left), the Topdown image (top-right), and the Noise image (bottom-left).

The images in Figure 20 were not part of the previous study. We created these images from principles of visual attention — in particular visual search - in order to obtain different reactions from participants when performing the PassPoints task as well as the Image Recall task. These images were selected for specific reasons. The Topdown image could potentially be useful in determining gaze patterns in a top-down visual search; in fact, we did not take this approach so the image simply illustrates the salience of the single red rectangle. The Noise image could potentially be useful in determining gaze patterns in a bottom-up visual search; and, the Figureground image could potentially be useful in determining where users look at an image when presented with two contrasting visual stimuli. 


\section{Participants}

Thirty-two participants were invited to participate in this research project. The gender of participants was deemed important for the scope of this research, therefore gender was a balanced variable for this experiment. Participants were recruited using the Carleton University Psychology Experiment Sign-up System (SONA), word of mouth, as well as postings on various bulletin boards throughout the university. The only prerequirement for each participant to be eligible to participate in this experiment was that he or she had not already taken part in testing sessions for another graphical password study which was being led by a fellow student experimenter in the HotLab. Age was not an important part of selection for this experimental project.

\section{Procedure}

The experiment in question was a between-subjects experiment. Each participant, upon having been given directions to the locale where the experimental testing was to be held, was first given a brief description of the experiment; then, he or she was asked to read and sign a consent form (see Appendix C). Each participant was assigned, at random, to one of two experimental conditions, A and B (explained in detail below). The participant was then asked to sit down in a chair in front of the Tobii eye tracker which, while recording all gaze points during the session, would also double as the screen where each participant would look and perform tasks for a significant component of the experiment. The experiment itself lasted approximately sixty minutes.

Before the experiment began the experimenter needed to calibrate the eye tracker device, so that the gaze points being recorded by the ClearView software package would 
be accurate for the participant in question during the particular testing session. The participant sat at a viewing distance that was between 40 and 70 centimetres away from the Tobii eye tracker screen (see Table 7 for viewing distances). The Tobii eye tracker ClearView Manual (Tobii Technology AB, 2006) recommends a viewing distance of $60 \mathrm{~cm}$. As allowed by the Tobii system, participants move during the experiment; enforcing a precise distance would have interfered with the participant's natural behavior. In terms of precision, the eye tracker measures accuracy in degrees. One degree of accuracy leads to an average error of approximately one centimetre between the intended and measured gaze point; this is measured under the condition that the participant in question sits at 50 centimetres from the screen. Also, the accuracy of the eye tracker can vary based on the conditions at the moment of testing: such conditions can be lighting, quality of calibration, and individual being tested. The average accuracy is claimed to be approximately 0.5 degrees using standard accuracy measurements for eye trackers (Tobii Technology AB, 2006).

There are also drift effects that may hinder the accuracy of the Tobii eye tracker. When a drift occurs, it indicates that there is deterioration of calibration for a particular individual. This can be due to changes in the characteristics of the eye, such as dryness or changes in lighting in the room, which are directly linked to the pupil of the eye. These drifts can vary from zero to two degrees for each eye individually. However, the Tobii eye tracker is claimed to have a way of counteracting these drift effects; through the use of binocular averaging, drift effects can be reduced to just about 0.5 degrees (Tobii Technology AB, 2006). 
In addition to drift effects, the need to compensate for head movement is crucial (since we are not controlling for this by having participants stabilize their head during testing). The eye tracker has another feature, head-motion compensation, which keeps track of all movement (including sideways, up or down, back and forth movements) resulting in a less than 1 degree of error across the entire field of view of the eye tracker camera.

For condition A, the PassPoints condition, the first stage of the experiment involved two trials in which each participant was introduced to the notion of graphical passwords. It was then explained to the participant that, during these two practice trials and any subsequent real trials, he or she would be asked to create a graphical password, always comprised of five different points on the image; this was to be their password. Each participant was instructed to attempt to create a graphical password that would be safe from anyone attempting to guess their password, just as any text-based password he or she had used in the past would be. Each participant was also told that there are three different, yet similar, parts to each trial: the creating of a graphical password, the confirming of that same graphical password and, finally, the logging in portion of the trial where the participant would be asked to repeat the exercise of entering his or her five chosen points comprising their graphical password. The participant was also informed that between the confirming of the password and the logging in section, a Mental Rotation Task (MRT) (Peters, 1995) would be given to them for each trial; this was done so that the participant would be distracted from thinking about their password for approximately thirty seconds. After having asked the participant if he or she had any questions concerning the directives provided to them relating to the testing session, a 
sample image was shown to the participant: the image depicts two dogs of a similar breed walking on a lawn as background. Five points on the image are outlined by an orange box, measuring approximately nineteen pixels in width by nineteen pixels in height. This was explained as being the margin of "error", or tolerance surrounding any of their five choices of clicks comprising their graphical password; this means that, upon confirming their password and/or logging into the system, any of the five points they will have clicked on needs to be within that pre-determined "error" area surrounding the original single-pixel click.

The participant then began the trials. Each trial consisted of three separate phases: the create phase, where the participant in question would select his or her 5 click-points comprising their graphical password; the confirm phase, where the system would have the participant re-enter, thus confirming, the same graphical password from the create phase; and, after the MRT distraction task, the login phase, where the participant would enter, for the last time, the same graphical password used in the first two phases.

If the participant in question did not click within the invisible tolerance box, in the confirm or login phase, then the software application returned an error message, indicating that the password is not the correct one. In these cases, the participant was given three choices: 1 - the participant could retry entering the correct password; 2 - the participant could reset the trial entirely, which would bring him or her to the password creation page for that same trial, thus allowing him or her to begin anew; 3- the participant could decide to skip the trial in its entirety if he or she felt that selecting a graphical password on the particular image in question was too difficult. 
The Tobii eye tracker recorded all fixation data for each of the trials 1 through 10 , including all three phases, for each participant. For our analysis, we later extracted only the data for the creation phase for trials 3 through 10 , as we regarded trials 1 and 2 as practice.

For condition B, the Image Recall condition, we wanted to capture eye gaze fixation data during a general task uninfluenced by the PassPoints method. We chose a simple memory task structured in the same way as the PassPoints condition. The researcher explained that ten different images, in total, would be shown over the sixtyminute session. It was explained that each image would be shown for thirty seconds, during which time the participant was asked to look at the image until it disappeared; when the image disappeared, the participant was asked to describe the image to the researcher for about fifteen seconds. We chose thirty seconds as the duration for each phase based on the approximate average creation time used by participants in Chiasson et al.'s (2007) study. Immediately following this exercise, the same image would once again be shown to the participant, and he or she would be asked to stare at it once more for approximately thirty seconds; next, two usability questions were shown on the screen, and the participant was asked to answer them using the mouse provided. The Mental Rotation Task was then administered to the participant; upon completion of the MRT, the participant would click on the "continue" button that appeared onscreen, and describe the same image one last time to the researcher. These phases correspond to the similar phases in the PassPoints condition. The Tobii eye tracker would record all fixation data for each participant and later we extracted the data for the first phase of each trial for trials 3 through 10 . 
After completion of the two practice trials and a few actual trials (approximately thirty minutes into the testing session) for either condition, the researcher instructed the participant that a five-minute break would be taken in order to fill out a demographics questionnaire (see Appendix E). The intent for the questionnaire was simply to gather information on each participant, so we would be better able to understand any usability problems that arose. Trial testing for either condition resumed after the questionnaire was filled out by the participant in question.

Upon completion of that last trial, the participant was asked to fill out a post-task questionnaire (see Appendix $\mathrm{J}$ and $\mathrm{K}$ ), which asked the participant to indicate to what degree they agreed or disagreed with various statements concerning usability and security issues relating to graphical passwords (for condition 1) and image memory (for condition 2). Upon completion of this questionnaire, the participant was given a debriefing form (see Appendix $\mathrm{H}$ and I), providing information concerning the goals of the study, as well as contact information for the main researcher and specific contact information for the ethics committee members.

Once the participant had read the debriefing form, he or she was asked to sign an "Honorarium-Received" ethics form, indicating they had received the honorarium for the study session; this step was skipped if the participant was to receive course credit for their participation in the study. The participant was then thanked for his or her help with the study, whereby they were free to leave the testing locale. 


\section{Changes to the Data Sets}

Before being able to apply any statistical analysis to our data set, it was necessary to make changes to the gaze data sets for each of the images. As the gaze data was set in an 800 by 600 pixel window, it was necessary to make changes to be able to reestablish the origin (i.e. point at the 0,0 coordinates) of the top-left of the images, which were 451 pixels in width and 331 pixels in height. This basically extracts the rectangle that contained the images, allowing us to conduct the analysis on only the gaze data points that were found within the frame of the PassPoints images. The basic data in all cases consists of fixation locations and durations. We partitioned the fixation data into areas of interest, determined by the tolerance of PassPoints, and aggregated the position and duration.

\section{User Feedback Data}

During the experimental stages of our study, we asked participants to fill out a post-task questionnaire (see Appendix J and K), which allowed us to gather feedback from users on their experience performing either the PassPoints task, or the Image Recall task. The questionnaires consisted of 32 Likert-scale type questions, the questions being unique to each of the two experimental conditions. This was done in an effort to ensure usability, get feedback from all participants in the study, and to ensure that there were no unexpected usability problems with the software. This information does not specifically relate to our hypotheses. 


\section{Analysis}

Upon having completed the experiment, we conducted the analysis of the resulting data. We examined each hypothesis, in turn, and determined statistical tests that could be used in order to adequately test that particular hypothesis.

To reiterate, the first hypothesis is, $\mathrm{H} 1 \mathrm{~A}$ is: 'A left to right horizontal trend exists on the $X$ axis of the gaze pattern data'; and, hypothesis H1B is: 'A top to bottom vertical trend exists on the Y axis of the gaze pattern data'. We wanted to test the validity of these hypotheses on our gaze distribution data, as Chiasson et al. (2008) found up-to-down and left-to-right trends among the click-points that users selected in creating graphical passwords. These trends were seen irrespective of the image used. In order to test this hypothesis, we use linear regression relating the $\mathrm{X}$ and $\mathrm{Y}$ distances to the ordinal values of the corresponding click-points.

The second hypothesis is, H2A, is: 'Fixation dwell-times decrease over the number of trials within subjects'; and, hypothesis $\mathrm{H} 2 \mathrm{~B}$ is: 'The number of fixations decreases over the number of trials within subjects'. The reasoning behind these hypotheses is the possibility that, as users become used to a particular task, they will develop strategies for selecting their click-points. This would thus reduce the time needed to perform each successive trial within the experiment, leading to a decrease in dwelltimes and number of fixations. We speculate that users make use of bottom-up visual search when first presented with an image at the very beginning of the task at hand; then, as they become familiar with the task and begin to develop strategies for selecting their click-points, their visual search transitions from a bottom-up to a top-down process. We 
expect to see this transition occurring as the trials progress for each participant in the experiment.

In order to test this hypothesis, we order each participant's trials, calculate the number of fixations for each image, and test for the existence of a trend showing a reduction of fixations per image. We use linear regression to test this hypothesis, in order to relate the number of fixations (dependent variable) with trial number (independent variable).

The third hypothesis, H3, is: 'Fixation point patterns resemble click patterns for the same picture across subjects'. The way we will test if this is true is first to gather all of the click-points data sets from all participants for one image, as well as the eye gaze data sets gathered via the eye tracker for the same image. We will then map the patterns for each of the two variables (i.e. click-points and gaze points), and determine similarity by looking at the images. We will next use the gaze data to construct dictionaries to guess the click-data following the method of Thorpe \& van Oorschot (2007). Finally, we use statistics developed by Van Lieshout \& Baddeley (1999), which are typically intended to compare spatial patterns in environmental studies. We use these statistics with a Monte Carlo technique to determine the significance of comparison.

\section{Results}

In this section, we first present a set of descriptive statistics to summarize participant behavior in our experiment. We then state each hypothesis in turn and look at each one individually. We present and discuss the data that were used in order to 
determine the validity of each hypothesis. We then examine the data through the use of graphs, tables, and statistical analyses.

\section{Descriptive Statistics for the PassPoints Condition}

We calculated descriptive statistics in order to better understand the usage of the PassPoints system when we proceed to the analysis stage of our study. As only the PassPoints condition asked participants to select click-points as part of their graphical password creation task, descriptive statistics for password creation, confirmation, and login are based solely on those data sets. The following tables break down the descriptive statistics by trial number, by image, and by overall times required to complete each of three phases: create, confirm, and login (the complete listing of tables can be found in Appendix L).

Table 9. Overall times in seconds for trials 3 through 10.

\begin{tabular}{l|cccccc}
\hline Phase & Minimum & $1^{\text {st }}$ Quartile & Median & Mean & $3^{\text {rd }}$ Quartile & Maximum \\
\hline Create & 4.891 & 16.69 & 26.2 & 30.33 & 39.18 & 108.9 \\
Confirm & 6.047 & 9.012 & 10.38 & 12.29 & 12.19 & 99.16 \\
Login & 6.703 & 9.281 & 10.42 & 11.37 & 12.94 & 22.69 \\
\hline
\end{tabular}

Table 10. Times in seconds for the Cars image.

\begin{tabular}{l|cccccc}
\hline Phase & Minimum & $1^{\text {st }}$ Quartile & Median & Mean & $3^{\text {rd }}$ Quartile & Maximum \\
\hline Create & 8.656 & 14.04 & 17.98 & 25.63 & 36.73 & 53.83 \\
Confirm & 6.047 & 8.824 & 10.07 & 10.54 & 11.39 & 19.95 \\
Login & 6.703 & 9.234 & 10.09 & 11.61 & 11.69 & 20.5 \\
\hline
\end{tabular}


Table 11. Times in seconds for the Figureground image.

\begin{tabular}{l|cccccc}
\hline Phase & Minimum & $1^{\text {st }}$ Quartile & Median & Mean & $3^{\text {td }}$ Quartile & Maximum \\
\hline Create & 4.891 & 14.88 & 20.35 & 23.67 & 31.02 & 50.5 \\
Confirm & 7.594 & 9.043 & 10.59 & 12.42 & 12.52 & 31.77 \\
Login & 7.062 & 8.863 & 9.594 & 10.01 & 10.26 & 18.91 \\
\hline
\end{tabular}

Table 12. Times in seconds for the Topdown image.

\begin{tabular}{l|cccccc}
\hline Phase & Minimum & $1^{\text {st }}$ Quartile & Median & Mean & $3^{\text {rd }}$ Quartile & Maximum \\
\hline Create & 10.98 & 14.62 & 22.95 & 24 & 26.53 & 69.58 \\
Confirm & 6.062 & 7.562 & 9.602 & 9.717 & 11.11 & 16.88 \\
Login & 6.797 & 9.305 & 10.37 & 10.48 & 11.48 & 17.19 \\
\hline
\end{tabular}

Table 13. Times in seconds for the Noise image.

\begin{tabular}{l|cccccc}
\hline Phase & Minimum & $1^{\text {st }}$ Quartile & Median & Mean & $3^{\text {rd }}$ Quartile & Maximum \\
\hline Create & 14.69 & 27.49 & 30.94 & 41.79 & 56.83 & 108.9 \\
Confirm & 9 & 11.34 & 11.86 & 18.11 & 14.81 & 99.16 \\
Login & 10.14 & 11.16 & 13.48 & 13.79 & 14.8 & 22.69 \\
\hline
\end{tabular}

Table 14. Times in seconds for the Paperclips image.

\begin{tabular}{l|cccccc}
\hline Phase & Minimum & $1^{\text {st }}$ Quartile & Median & Mean & $3^{\text {rd }}$ Quartile & Maximum \\
\hline Create & 19.08 & 26.21 & 35.8 & 39.07 & 45.41 & 92.17 \\
Confirm & 7.062 & 10.57 & 11.8 & 13.05 & 12.38 & 27.92 \\
Login & 7.547 & 10.36 & 12.88 & 12.72 & 13.69 & 20.48 \\
\hline
\end{tabular}

The data provided in Tables 9 through 14 indicates that some of the images stand out as requiring participants in the PassPoints condition to take longer times to perform their task when compared to the other images. Such examples of this are the Noise and Paperclips images, which had a mean creation time of 41.79 seconds and 39.07 seconds, respectively. These seem to indicate longer creation times when compared to such images 
as the Figureground and Topdown images, which had mean creation times of 23.67 seconds and 24.00 seconds, respectively.

An Analysis of Variance (ANOVA) was done on each of the creation and login times for all eight images, to see if there were any meaningful differences between them. The results of the ANOVA on the create times demonstrate that there was significance between the images, with $F(7,105)=5.721, \mathrm{p}<0.001$. The results of the ANOVA on the login times demonstrate that there was significance between the images, with $F(7,82)=$ $02.3791, \mathrm{p}=0.0289$. Figures 21 and 22 below show the boxplots for the create and login phases, respectively. The results for these phases suggest that the type of image may play a role in the time required to complete the task. This could be due to the complexity of the image, the lack of patterns, objects, or forms participants could potentially use as click-points, or a variety of other factors. We speculate that longer create times might also indicate images where users were less likely agree on click-points, leading to clickpoints that were harder to guess. 
Create phase completion times

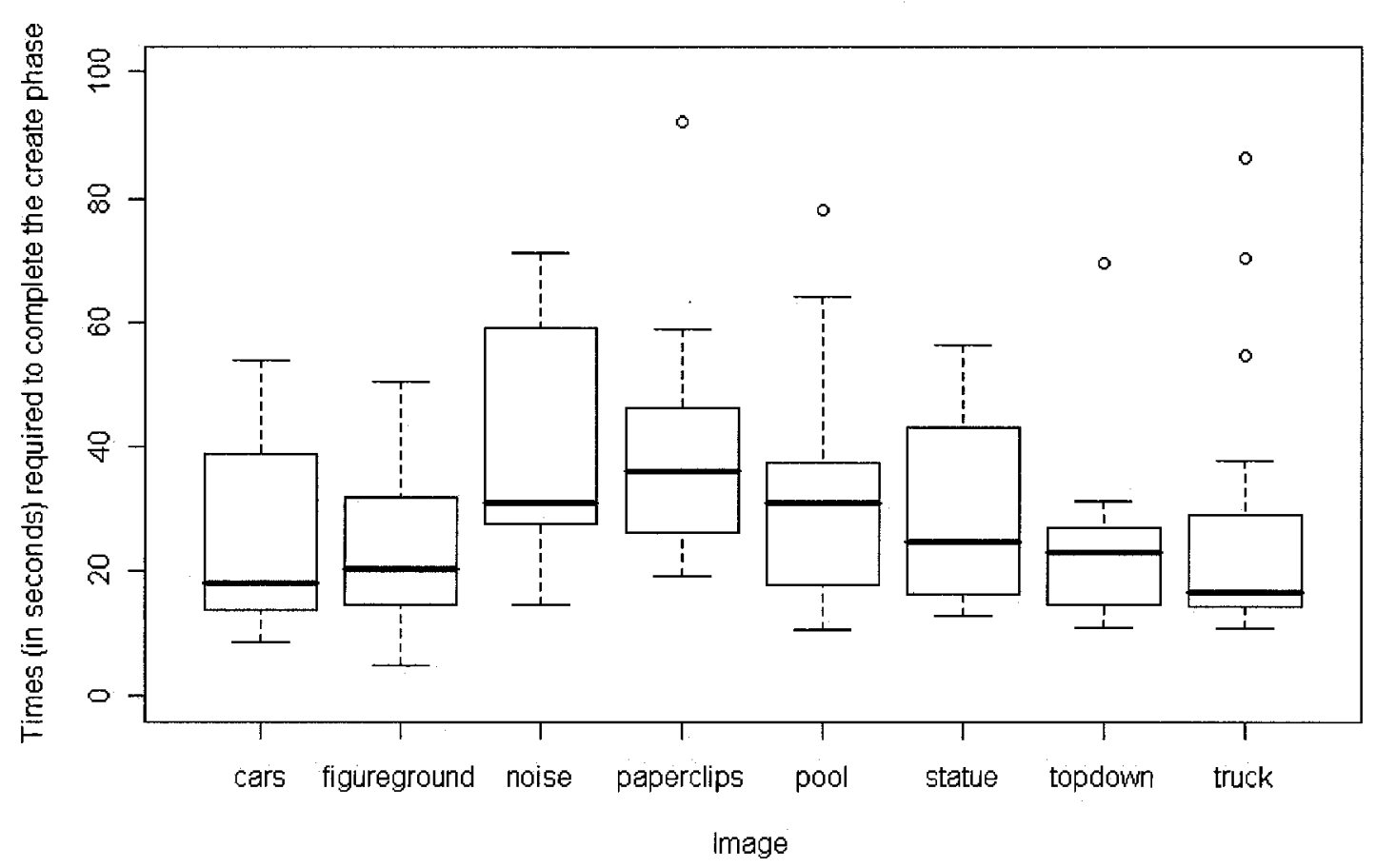

Figure 21. Create phase completion times. Each of the create times is depicted in boxplot form for each of the eight images used in our study. 


\section{Login phase completion times}

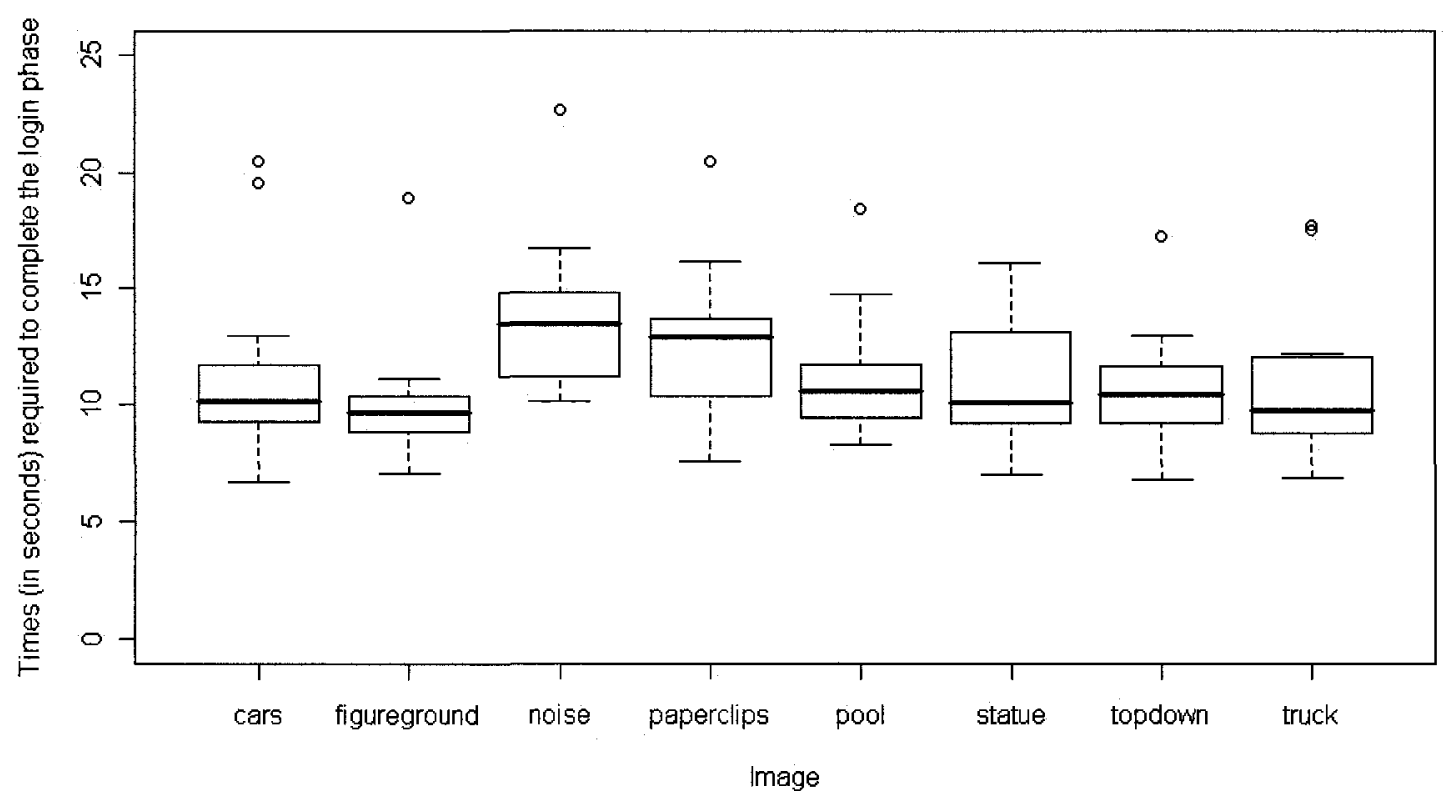

Figure 22. Login phase completion times. Each of the login times is depicted in boxplot form for each of the eight images used in our study.

\section{Hypotheses}

\section{Hypothesis 1:A and B}

"A left to right horizontal trend exists on the X axis of the gaze pattern data" and "A top to bottom vertical trend exists on the $\mathrm{Y}$ axis of the gaze pattern data" form the first hypothesis. For this hypothesis, we were interested to see if there existed any left to right, or top to bottom trends among the data. It was hypothesized that there existed such trends among the participant gaze data, as a colleague had previously found these trends to exist among click-points data. We found no such trends among gaze distributions of either the PassPoints or Image Recall task conditions. We can see this by examining the following graphs: Figures 23 and 25 depict the gaze time ratios on the $\mathrm{X}$ axis, while Figures 24 and 26 represent these same ratios on the $\mathrm{Y}$ axis. By gaze time ratio we mean the time that the 
gaze fixation begins relative to the overall duration of the creation phase. For example, 0.5 would indicate a gaze fixation that begins halfway through the creation phase. This accommodates differences in the creation durations for different participants. In these and later graphs and analyses, we only consider trials 3 through 10, because trials 1 and 2 were practice trials to familiarize the participant with the system. We also ran a regression analysis on each of the data sets separately, in order to see if there was any significance of the slope in each case. We report on this in the sections immediately below. 


\section{Gaze time ratios on the $X$ axis for the PassPoints condition}

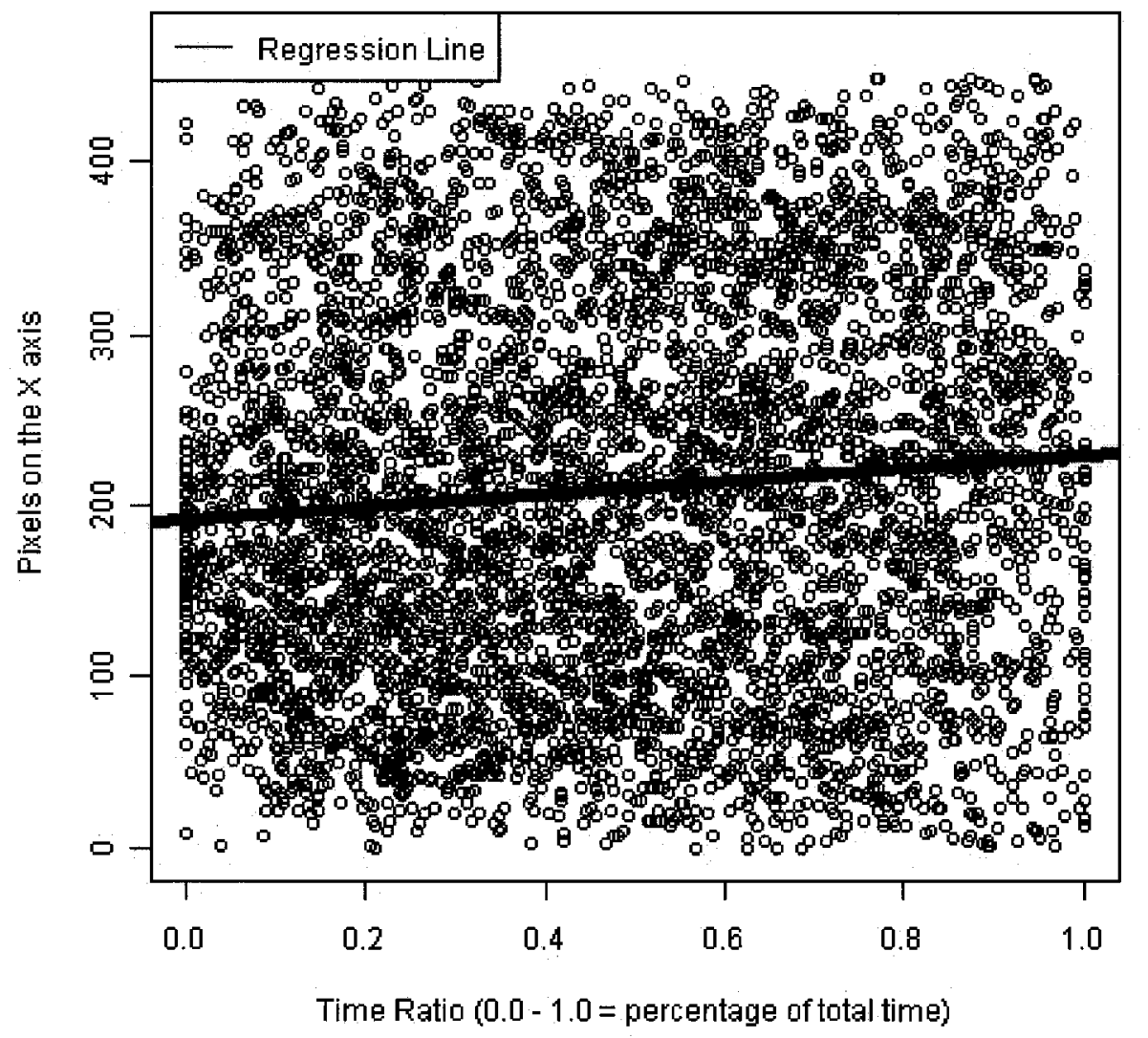

Figure 23. The gaze time ratios on the $\mathrm{X}$ axis for all gaze in the PassPoints condition, with pixels on the $X$ axis plotted as a function of time ratio.

In Figure 23, the PassPoints condition, it is not easy to see any visible left to right pattern of the data; however, we can see that many participants spend more time looking at the left portion of the image much earlier during the trials. If there was a left to right pattern in the data, we would expect to see a different vertical pattern from left to right on the graph. We see this informally by looking at the higher density of points in the graph, located between the 0 to 200 pixels on the $\mathrm{Y}$ axis, and the 0.0 to 0.4 on the $\mathrm{X}$ axis; the higher density here indicates that participants spent more of the earlier stages of the trials looking at the first 200 pixels of left side of the image, along the $\mathrm{X}$ axis. The regression 
analysis for this data set tells us that the residuals have skewness $=0.157(\mathrm{SE}=0.033)$, kurtosis $=-0.967(\mathrm{SE}=0.066)$, and shows significance, with the slope $p$ value $<.001$, $r(1,5380)=0.0916$, and the adjusted $r^{2}=0.0084$. This regression analysis indicates that the slope is significant, but that there is no strong correlation among the variables, as we can see from the adjusted $\mathrm{r}^{2}$ value, which is very close to zero.

\section{Gaze time ratios on the $Y$ axis for the PassPoints condition}

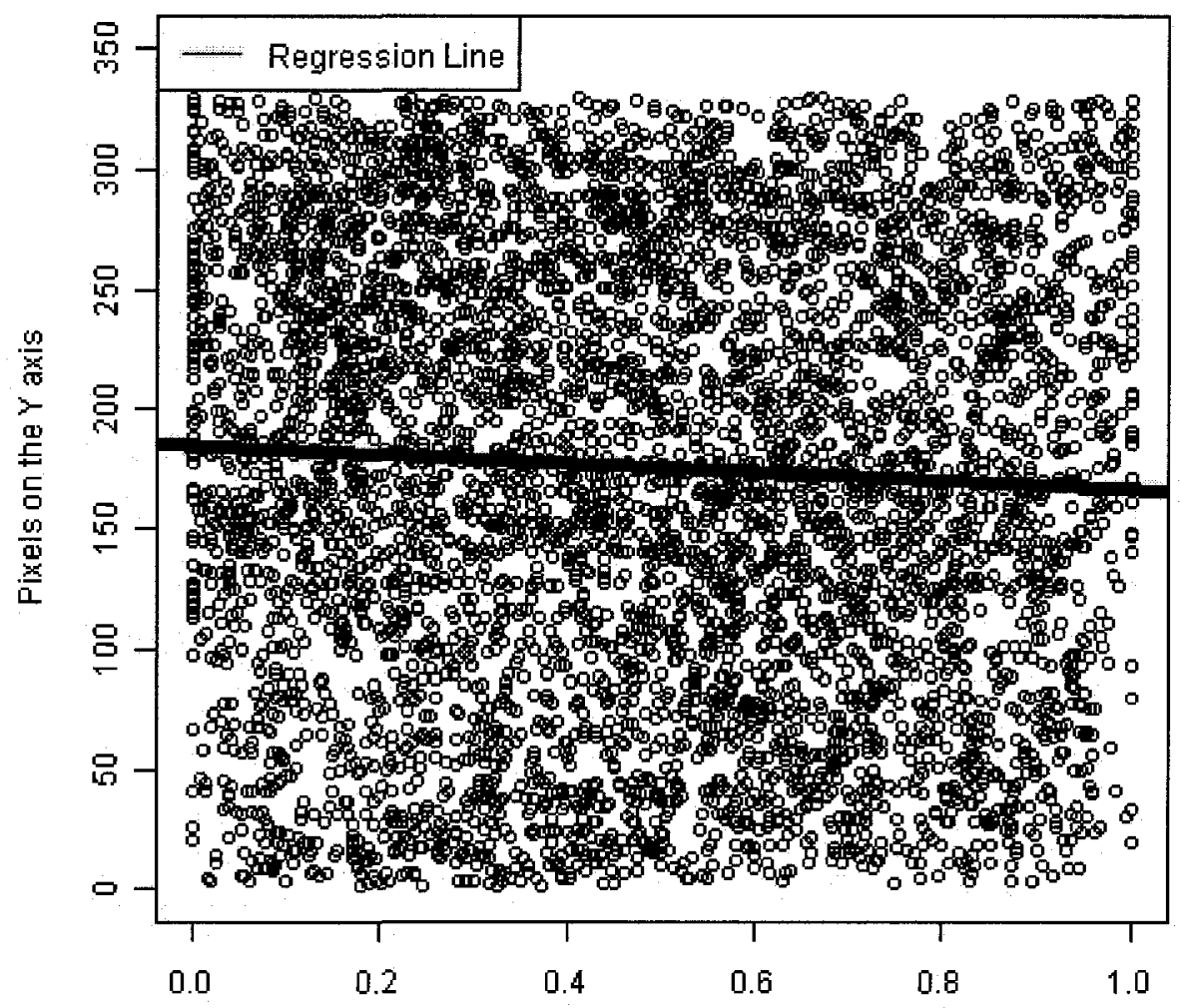

Time Ratio $(0.0-1.0=$ percentage of total time $)$

Figure 24. The gaze time ratios on the $Y$ axis for all gaze in the PassPoints condition, with pixels on the $Y$ axis plotted as a function of time ratio.

Figure 24 does not show a top to bottom trend, but may show a slight tendency of participants looking toward the middle of the image in the earlier seconds of each trial.

We can see the emphasis on the middle of the image by looking at the density in the 
graph, between approximately the $210^{\text {th }}$ pixel to the $330^{\text {th }}$ pixel on the $\mathrm{Y}$ axis of the graph; this indicates that participants looked at the middle portion of the image in the earlier stages of the trial. The emphasis on the middle was not explored further as it did not relate directly to our hypothesis. Considering our hypothesis about the trend from top to bottom, the regression analysis for this data set tells us that the residuals have skewness $=-0.158(\mathrm{SE}=0.033)$, kurtosis $=-1.121(\mathrm{SE}=0.067)$, and shows significance, with the slope $p$ value $<.001, r(1,5380)=0.051$, and the adjusted $r^{2}=0.0026$. This analysis indicates that the slope is significant but, once again, the adjusted $r^{2}$ is very close to zero, indicating a very poor correlation between variables. 


\section{Gaze time ratios on the $X$ axis for the Image Recall condition}

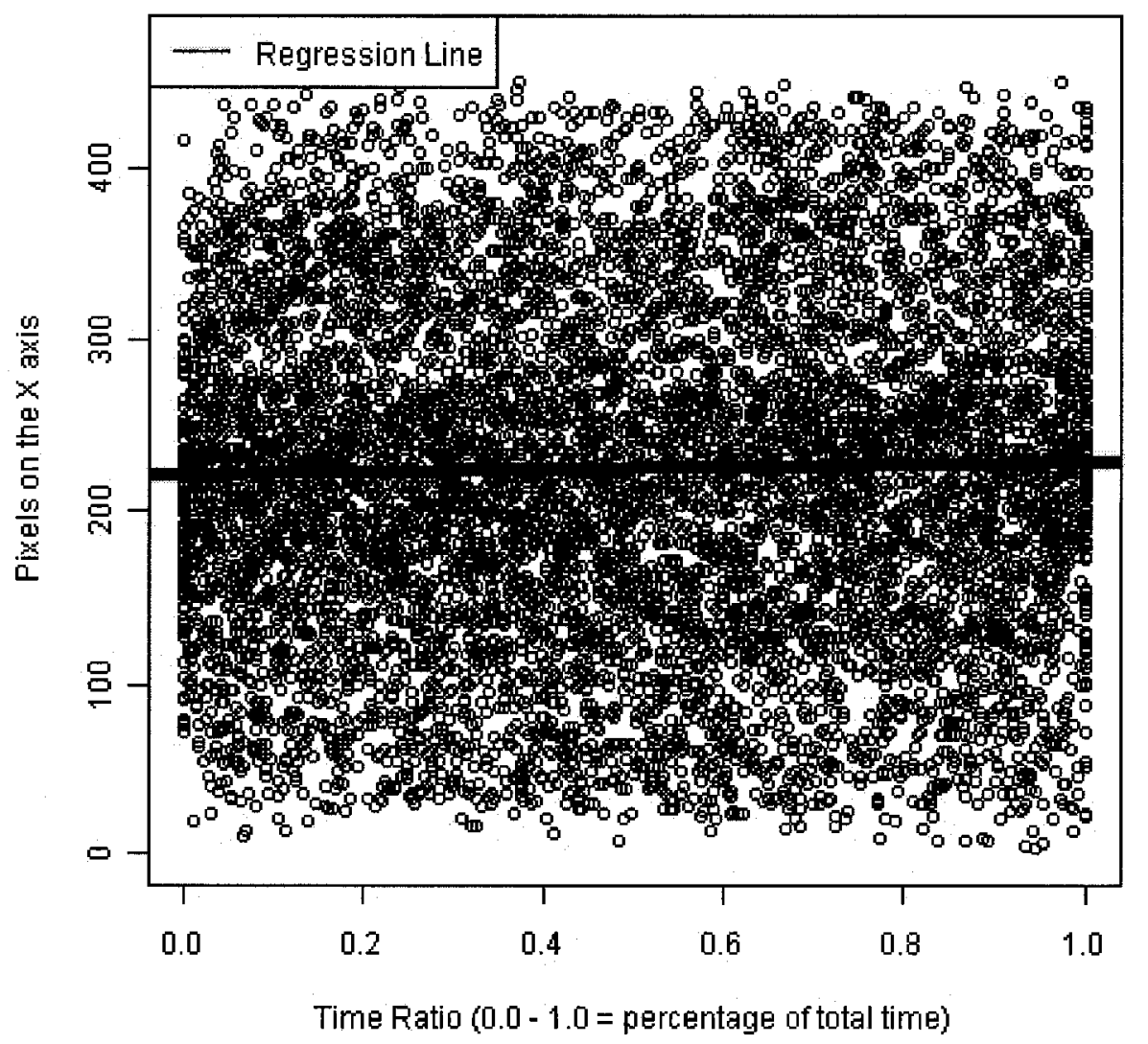

Figure 25. The gaze time ratios on the $\mathrm{X}$ axis for all gaze in the Image recall condition, with pixels on the $\mathrm{X}$ axis plotted as a function of time ratio.

In Figure 25, the Image Recall condition, we do not see any left to right trend. We can see that participants tend to look at the middle of the image much more often than anywhere else on the image for the entire duration of the trials, despite no instruction to do so specifically. We can see this informally by looking at the density of the graph, between approximately the $150^{\text {th }}$ pixel to the $270^{\text {th }}$ pixel on the $\mathrm{X}$ axis, during the entire time for each trial. The regression analysis for this data set tells us that the residuals have skewness $=0.062(\mathrm{SE}=0.027)$, kurtosis $=-0.749(\mathrm{SE}=0.054)$, and shows no significance, with the slope $p$ value $=0.0576, r(1,8201)=0.017$, and the adjusted $r^{2}=$ 
0.0003. In this case, the adjusted $\mathrm{r}^{2}$ is extremely small and close to zero, which indicates almost no relationship whatsoever between the variables.

\section{Gaze time ratios on the $Y$ axis for the Image Recall condition}

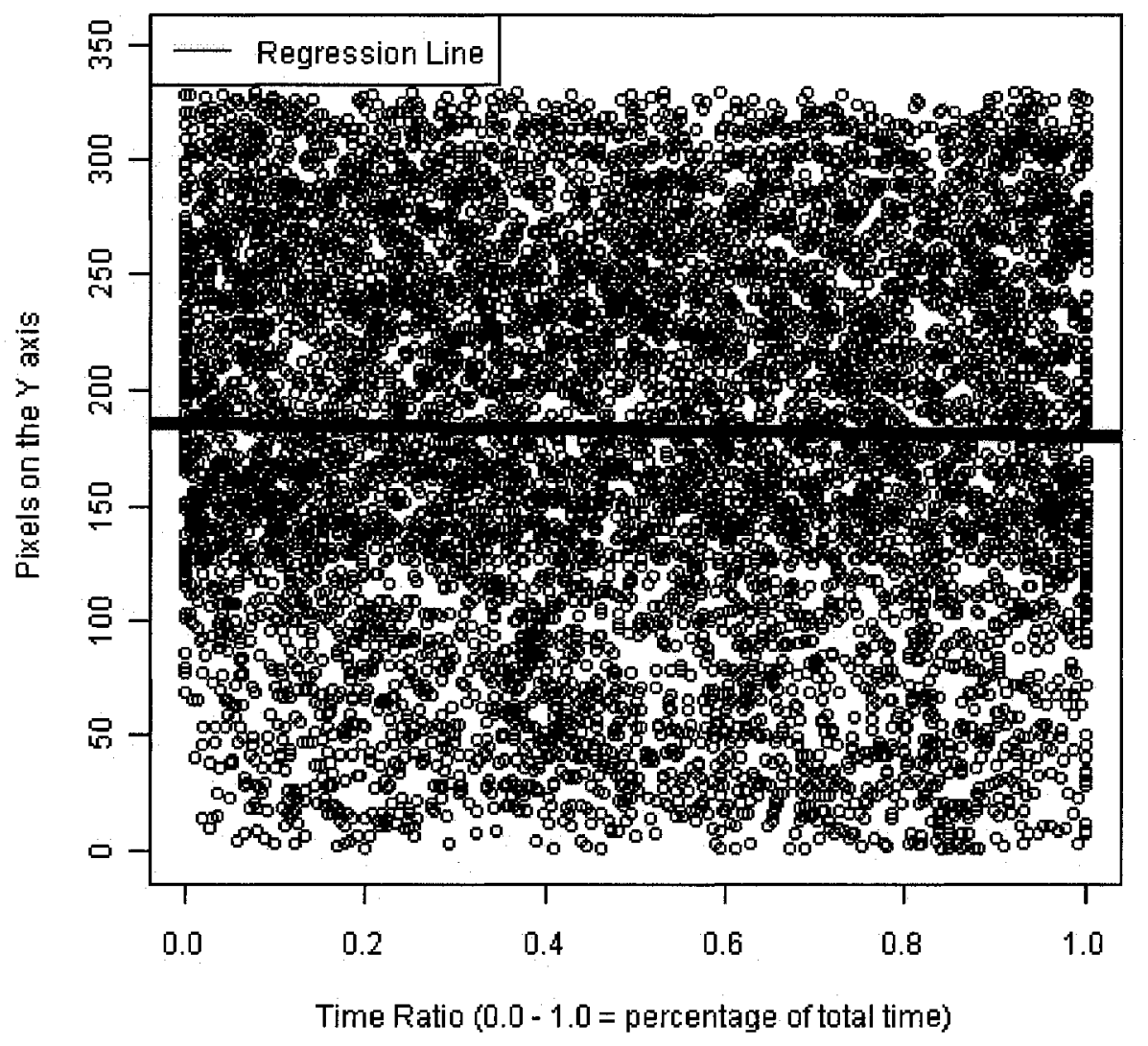

Figure 26. The gaze time ratios on the $\mathrm{Y}$ axis for all gaze in the Image Recall condition, with pixels on the $\mathrm{Y}$ axis plotted as a function of time ratio.

Figure 26 does not demonstrate the expected top to bottom trend, but does show a slight tendency for participants to gaze more toward the middle and bottom portions of the image during a large part of the time spent on each of the trials. We can see this informally by looking at the graph density between the $120^{\text {th }}$ pixel and the $170^{\text {th }}$ pixel for the middle of the image, and between the 210th pixel and the $300^{\text {th }}$ pixel for the bottom of the image, along the $\mathrm{Y}$ axis. The increased density at these two sections of the graph 
indicates that participants spent their time looking at these sections of the images, during all trials. The regression analysis for this data set tells us that the residuals have skewness $=-0.253(\mathrm{SE}=0.027)$, kurtosis $=-0.883(\mathrm{SE}=0.054)$, and shows no significance, with the slope $p$ value $=0.0694, r(1,8201)=0.017$, and the adjusted $r^{2}=0.0003$. In this case, the slope $p$ value is almost at a significant value, but the adjusted $\mathrm{r}^{2}$ is a very small number, almost zero, which indicates almost no relationship between variables.

As we can see from the resulting analysis, there is no evidence to suggest that participants looked at each image in a left to right or top to bottom trend in either condition. We speculated we might see this trend due to the fact that Chiasson et al. (2008)'s study on graphical passwords did indeed see these trends among click-points data. Their data consisted solely of click-points and not gaze distribution data. Our data suggests that people may in fact look at images in a different way than their click patterns show.

\section{Hypothesis 2: $A$ and $B$}

The second hypothesis is: "Fixation dwell-times decrease over the number of trials within subjects" and "The number of fixations decreases over the number of trials within subjects." For this hypothesis, we were interested to see if there was a decrease in the dwell times, or in the number of fixations, exhibited by participants over the number of trials for each of the conditions. It was hypothesized that there existed such trends among the participant gaze data, as we believed that participants would get used to the task at hand over successive trials, and would thus fixate less, and for shorter periods of time, as each successive trial passed. We found no evidence for this hypothesis. 
Participants did not tend to fixate significantly less, nor did they dwell significantly less as the trial number increased. We can see this by examining the following graphs:

Figures 27 and 28 depict the gaze fixation durations as a function of trial number for the PassPoints and Image Recall tasks, respectively, while Figures 29 and 30 represent the number of fixations as a function of trial number for these same conditions. The gaze fixation duration is calculated by the Tobii eye tracker, and is depicted in milliseconds. The data for each trial consists of all gaze distribution data for all participants for each trial, for each condition. Each of the graphs represents the data from 16 participants for the condition in question (PP or IR). We ran a regression analysis on each of the data sets, and we present the results below. 


\section{Gaze fixation durations plotted by trial for the PP condition}

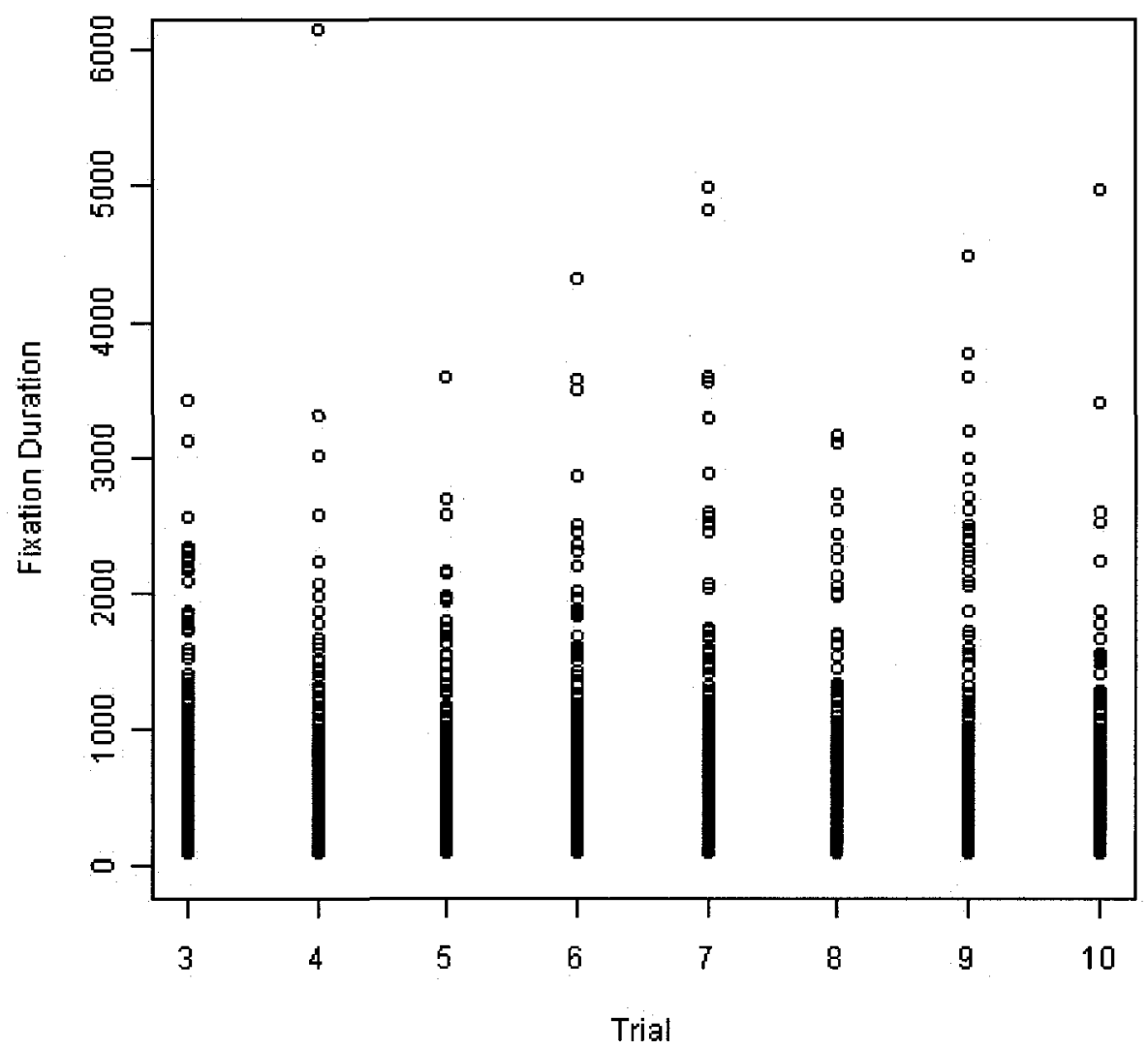

Figure 27. Gaze fixation durations for the PassPoints condition, plotted as fixation duration (ms) as a function of trial number.

Figure 27 shows the data for the PassPoints condition, and we cannot see any significant reduction of the duration of fixations as the trial number increases. The distributions were not normally distributed, with skewness $=4.502(\mathrm{SE}=0.03)$ and kurtosis $=30.958(\mathrm{SE}=$ 0.06); therefore, we applied a transformation taking the logarithm of the duration data. The regression analysis for this data set tells us that the residuals have skewness $=0.943$ $(\mathrm{SE}=0.03)$, kurtosis $=0.819(\mathrm{SE}=0.06)$, and shows no significance, with the slope $p$ value $=0.958, r(1,6564)=0.012$, and the adjusted $r^{2}=0.0002$. The correlation among variables in this particular regression analysis is almost non existant, according to the 
adjusted $r^{2}$ value. We were basing our hypothesis on the assumption that participants would develop top-down search patterns in order to spend less time looking at the images, which would ultimately reduce the number of fixations as the trial number increased. As these results suggest, we have no evidence that this is the case. We found similar results for the analyses later in this section.

\section{Gaze fixation durations plotted by trial for the IR condition}

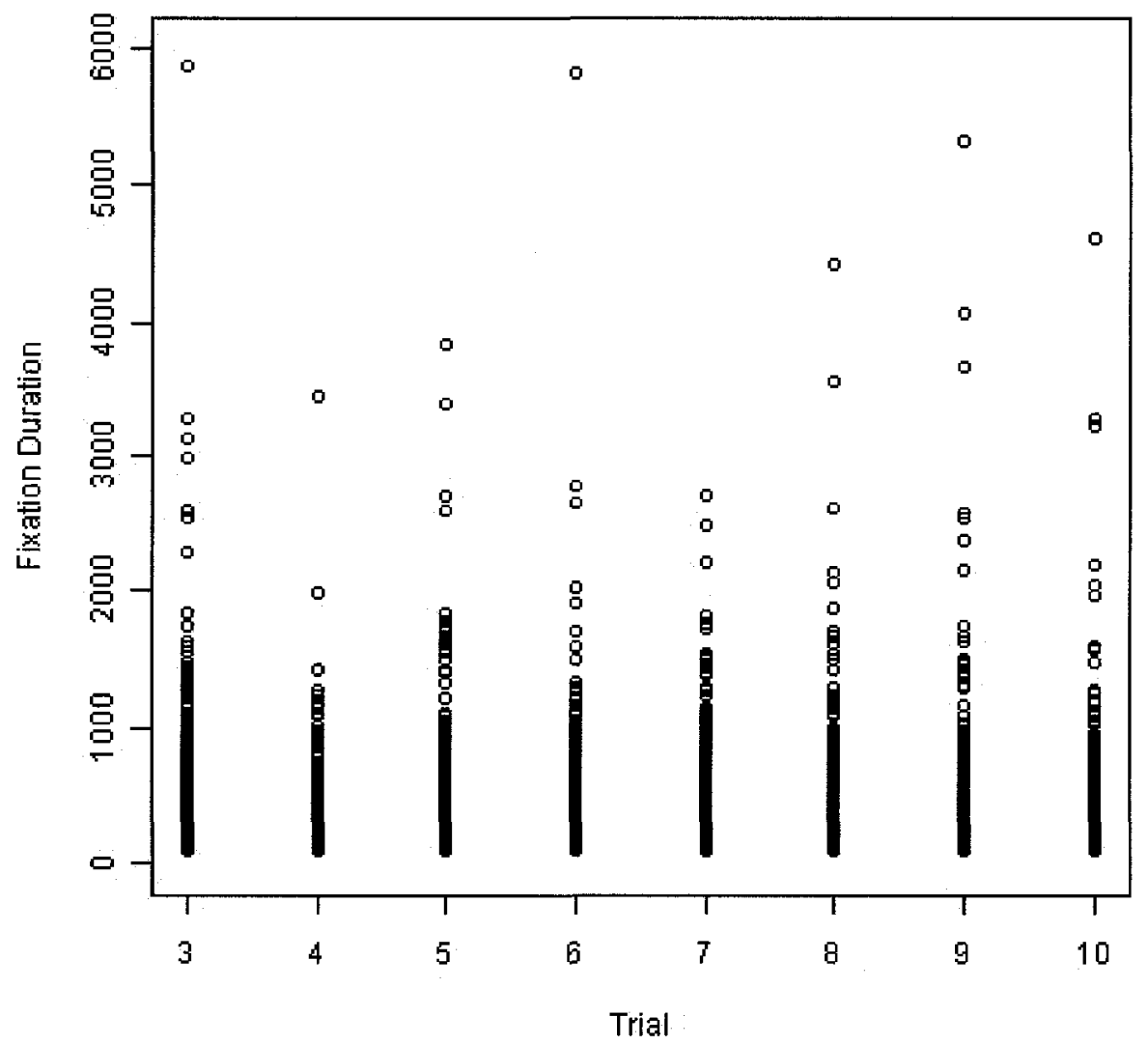

Figure 28. Gaze fixation durations for the Image Recall condition, plotted as fixation duration (ms) as a function of trial number.

Figure 28 shows the Image Recall condition, and again we cannot see any significant reduction of the duration of fixations as the trial number increases. The distributions were not normally distributed, with skewness $=11.76(\mathrm{SE}=0.026)$ and kurtosis $=256.9(\mathrm{SE}=$ 
053); therefore, we again applied a transformation taking the logarithm of the duration data. The regression analysis for this data set tells us that the residuals have skewness $=$ $0.776(\mathrm{SE}=0.026)$, kurtosis $=1.362(\mathrm{SE}=0.052)$, and still shows no significance, with the slope $p$ value $=0.06, r(1,8644)=0.017$, and the adjusted $r^{2}=0.0003$. It is apparent that the relationship between variables is not very strong, according to the adjusted $\mathrm{r}^{2}$ value, which is close to zero. Table 15 below shows the number of fixation points for both the PassPoints condition graph (Figure 28) and the Image Recall condition graph (Figure 29).

We also conducted a 2-way between-subjects analysis of variance to test for significant differences in gaze fixation duration and experimental condition (PassPoints and Image Recall) by trial number. The resulting ANOVA demonstrates that there was no significant difference between the two conditions, with $F(1,15196)=0.011, p=0.916$; there was a significant difference between trials, with $F(1,15196)=3.807, p<0.001$; and, there was a significant interaction between condition and trial number, with $F(1,15196)=4.036, p<0.001$. We speculate that these relationships arose as the participants were still getting used to the task, and their behavior was still developing over the course of the trials. 
Table 15. Number of gaze fixations for both the PassPoints and the Image Recall conditions, for trials 3 through 10.

\begin{tabular}{l|ll}
\hline Condition & Trial & \# of fixation points \\
\hline PP & 3 & 858 \\
PP & 4 & 835 \\
PP & 5 & 811 \\
PP & 6 & 864 \\
PP & 7 & 978 \\
PP & 8 & 771 \\
PP & 9 & 761 \\
PP & 10 & 688 \\
IR & 3 & 1023 \\
IR & 4 & 1129 \\
IR & 5 & 1071 \\
IR & 6 & 1010 \\
IR & 7 & 1114 \\
IR & 8 & 1087 \\
IR & 9 & 1058 \\
IR & 10 & 1154 \\
\hline
\end{tabular}




\section{Number of gaze fixations plotted by trial for the PP condition}

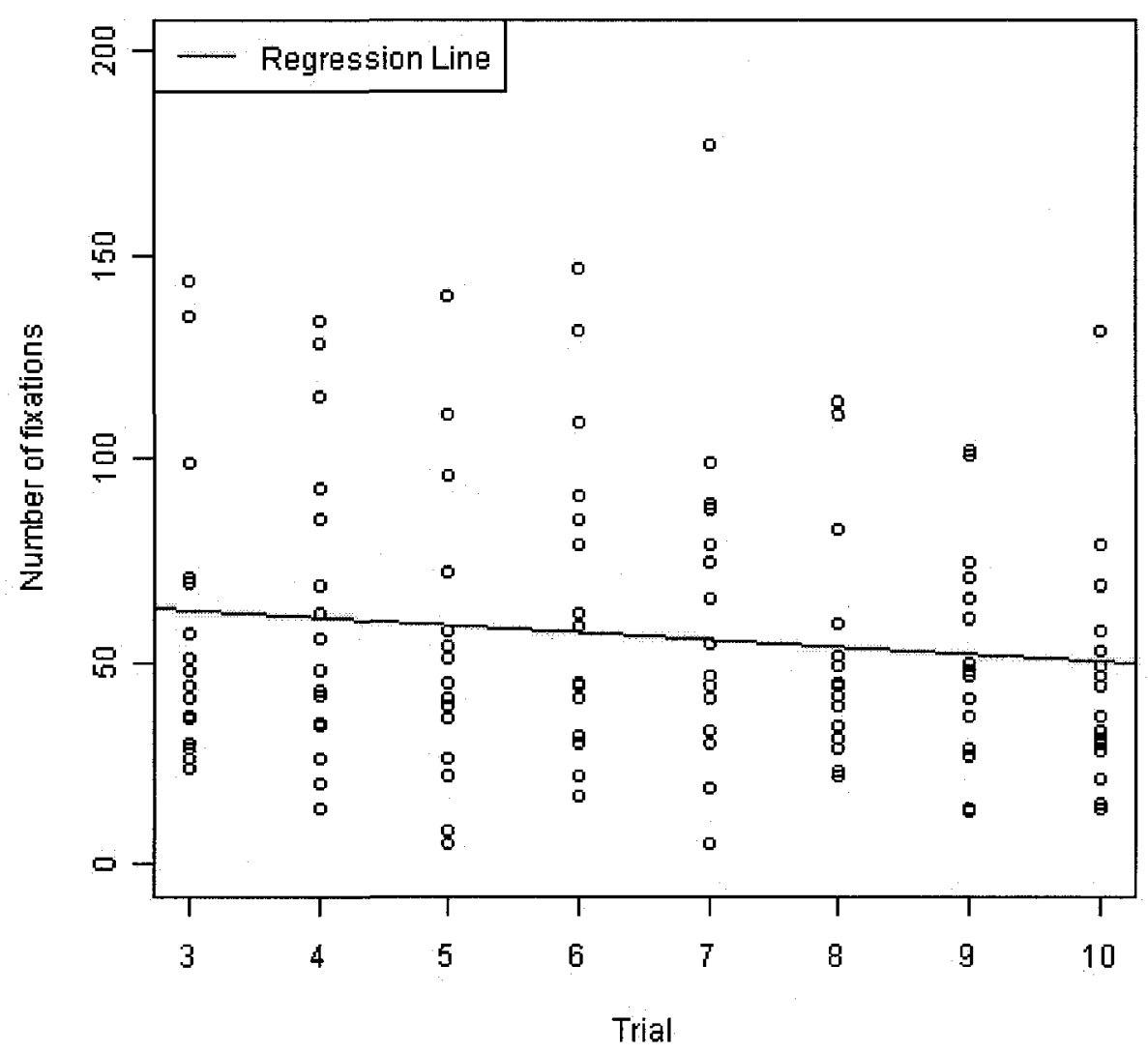

Figure 29. Number of gaze fixations for the PassPoints condition, plotted as number of fixations as a function of trial number. 16 participants from the PassPoints condition are plotted.

Hypothesis $2 \mathrm{~B}$ related to the number of fixations for each trial. We begin again with the PassPoints condition. We can see in Figure 29 that the line suggests a reduction in the number of fixations as the trial number increases. However, the regression analysis for this data set tells us that the residuals have skewness $=1.07(\mathrm{SE}=0.217)$, kurtosis $=$ $0.834(\mathrm{SE}=0.433)$, and shows no significance, with the slope $p$ value $=0.177, r(1,126)=$ 0.081 , and the adjusted $r^{2}=0.007$. With such a small adjusted $\mathrm{r}^{2}$, there is not enough variance accounted for to indicate a strong relationship between variables. We therefore cannot conclude that the number of fixations is strongly correlated with the trial number. 
We ran a repeated measures Analysis of Variance (ANOVA) on the fixation data, to see if there were any significant differences between the number of fixations per trial. The resulting ANOVA demonstrates that there were no significant differences between trial numbers, with $F(1,112)=0.3435, p=0.932$.

\section{Number of gaze fixations plotted by trial for the IR condition}

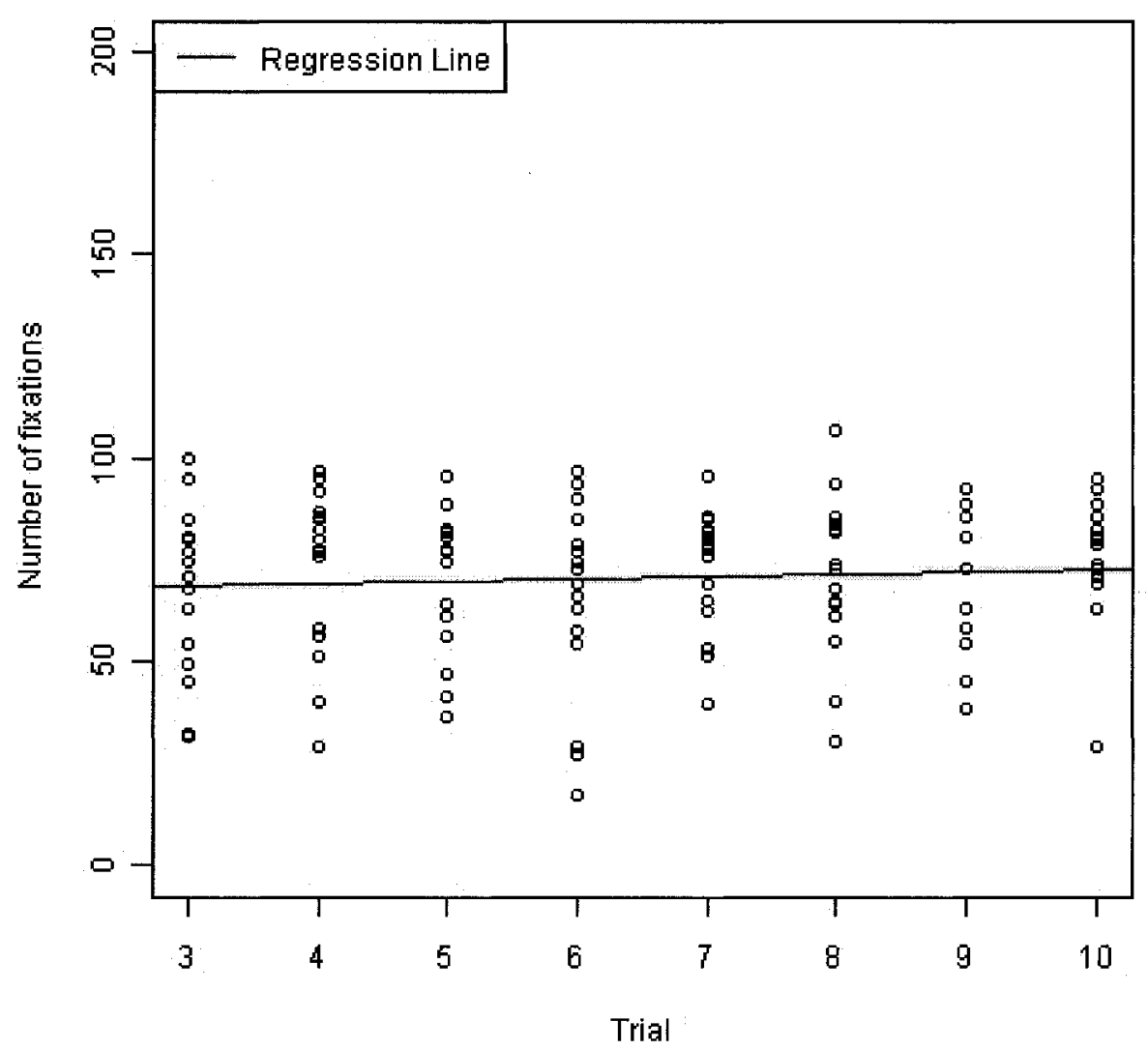

Figure 30. Number of gaze fixations for the Image Recall condition, plotted as number of fixations as a function of trial number. 16 participants from the Image Recall condition are plotted.

We can see in Figure 30 that there exists no significant reduction in the number of fixations as the trial number increases. The regression analysis for this data set tells us that the residuals have skewness $=-0.726(\mathrm{SE}=0.217)$, kurtosis $=-0.03(\mathrm{SE}=0.433)$, and shows no significance, with the slope $p$ value $=0.405, r(1,126)=0.049$, and the 
adjusted $r^{2}=0.002$. In this case, the adjusted $r^{2}$ is close to zero, and the slope $p$ value is far from being significant, therefore we cannot account for enough variance to indicate a strong correlation between the variables.

Like for the PassPoints condition, we ran a repeated measures Analysis of Variance (ANOVA) on the ImageRecall fixation data, to see if there were any significant differences between the number of fixations per trial. The resulting ANOVA demonstrates that there are no significant differences between trial numbers, with $F(1$, $112)=0.585, p=0.767$.

We also conducted a 2-way between-subjects analysis of variance to test for significant differences in gaze fixations and experimental condition (PassPoints and Image Recall) by trial number. The resulting ANOVA demonstrates that there was a significant difference between the two conditions, with $F(1,240)=15.758, p<0.001$; there was no significant difference between trials, with $F(7,240)=0.433, p=0.881$; and, there was no significant interaction between condition and trial number, with $F(7,240)=$ $0.775, p=0.609$. The difference between the conditions is to be expected because in the Image Recall condition participants always gazed for 30 seconds, whereas in the PassPoints condition it varied.

As we can see from the resulting data analysis and graphs, there is no evidence to support our hypothesis that the number of fixations, as well as their dwell times, decrease as the trial number increases. We can see, however, that the number of fixations per trial varies a great deal more in the PassPoints condition than in the Image Recall condition. This could be attributed to the notion that participants in the PassPoints condition focus 
quite a bit more on some images than they do on others, when attempting to select proper click-points that would render their graphical password more secure.

\section{Hypothesis 3}

The third hypothesis is: "Fixation point patterns resemble click patterns over the same picture across subjects". In order to test this hypothesis, we did several things. First, we produced density maps superimposed on the images and studied the overlap. Second, we calculated attack dictionaries based on the data and then applied them to sets of clickpoints. Third, we considered the JCross statistic for comparing point patterns.

\section{Image Analysis for Hypothesis 3}

For each of the eight images used in the study: Cars, Pool, Truck, Paperclips, Statue, Topdown, Figureground, and Noise, we overlaid each of the point patterns, including the old lab and field study click-points data of Chiasson et al., (2007) (where available), the thesis PassPoints condition click-points data, the PassPoints condition gaze data, as well as the Image Recall condition gaze data, onto their respective original image. We then compared each of the point patterns for an image with the naked eye.

For the images shown in Figures 31 through 38, we show the density maps overlaid on top of the gaze or click-points, which are then overlaid onto the original image. The density maps were created using the Spatstat package for the $\mathrm{R}$ statistical programming software (Baddeley \& Turner, 2005), using the density function for point patterns. The density maps are gradiated as follows: the red color indicates lesser dense areas, the yellow color indicates moderately dense areas, and the green color indicates the 
highest density areas. The density function used in the $\mathrm{R}$ statistical package divided each of the color regions by density range; the first density range was between 0.0 and 0.001 , the second was between 0.001 and 0.0075 , the third was between 0.0075 and 0.05 , and the fourth was between 0.05 and 1.0. These numbers relate to the intensity of each pixel relative to the total intensity of the image. Table 10 below summarizes the number of participants and points depicted in each of the images located within Figures 31 through 38.

Table 16. Number of participants within each of the images found in Figures 30 through 37.

\begin{tabular}{|c|c|c|c|}
\hline Image & Study & Number of participants & $\begin{array}{l}\text { Number of click } \\
\text { points or gaze } \\
\text { fixations }\end{array}$ \\
\hline \multirow[t]{5}{*}{ Cars } & A (PassPoints) & 16 & 910 \\
\hline & B (Image Recall) & 16 & 1197 \\
\hline & C (Field study) & 376 & 1880 \\
\hline & D (Old lab study) & 43 & 215 \\
\hline & E (New lab study) & 16 & 80 \\
\hline \multirow[t]{5}{*}{ Pool } & A (PassPoints) & 16 & 893 \\
\hline & B (Image Recall) & 16 & 1182 \\
\hline & C (Field study) & 376 & 1880 \\
\hline & D (Old lab study) & 43 & 215 \\
\hline & E (New lab study) & 16 & 80 \\
\hline \multirow{4}{*}{ Statue } & A (PassPoints) & 16 & 780 \\
\hline & B (Image Recall) & 16 & 1125 \\
\hline & C (Old lab study) & 43 & 215 \\
\hline & D (New lab study) & 16 & 80 \\
\hline \multirow[t]{4}{*}{ Truck } & A (PassPoints) & 16 & 929 \\
\hline & B (Image Recall) & 16 & 1192 \\
\hline & C (Old lab study) & 43 & 215 \\
\hline & D (New lab study) & 16 & 80 \\
\hline \multirow[t]{4}{*}{ Paperclips } & A (PassPoints) & 16 & 1197 \\
\hline & B (Image Recall) & 16 & 1178 \\
\hline & C (Old lab study) & 43 & 215 \\
\hline & D (New lab study) & 16 & 80 \\
\hline \multirow[t]{3}{*}{ Figureground } & A (PassPoints) & 16 & 712 \\
\hline & B (Image Recall) & 16 & 968 \\
\hline & C (New lab study) & 16 & 80 \\
\hline \multirow[t]{3}{*}{ Topdown } & A (PassPoints) & 16 & 786 \\
\hline & B (Image Recall) & 16 & 1269 \\
\hline & C (New lab study) & 16 & 80 \\
\hline \multirow[t]{3}{*}{ Noise } & A (PassPoints) & 16 & 1041 \\
\hline & B (Image Recall) & 16 & 920 \\
\hline & C (New lab study) & 16 & 80 \\
\hline
\end{tabular}


Figures 31 and 32 each contain 5 images; each of the images A through E depicts the main image as the backgrounds which, for these particular images, are Cars and Pool. For each of these two figures, image A shows the PassPoints eye gaze distribution data; image B shows the Image Recall gaze distribution data; image C shows the field study click-point data; image D shows the old lab study click-point data; and, image E shows the new lab study click-point data.

Figures 33, 34, and 35 each contain 4 images; each of the images A through D depicts the main images as the backgrounds which, for these particular images, are Statue, Truck, and Paperclips. For each of these three figures, image A shows the PassPoints eye gaze distribution data; image B shows the Image Recall gaze distribution data; image $\mathrm{C}$ shows the old lab study click-point data; and, image $\mathrm{D}$ shows the new lab study click-point data.

Figures 36, 37, and 38 each contain 3 images; each of the images A through $\mathrm{C}$ depicts the main images as the backgrounds which, for these particular images, are Figureground, Topdown, and Noise. For each of these three figures, image A shows the PassPoints eye gaze distribution data; image B shows the Image Recall gaze distribution data; and, image $\mathrm{C}$ shows the new lab study click-point data. 


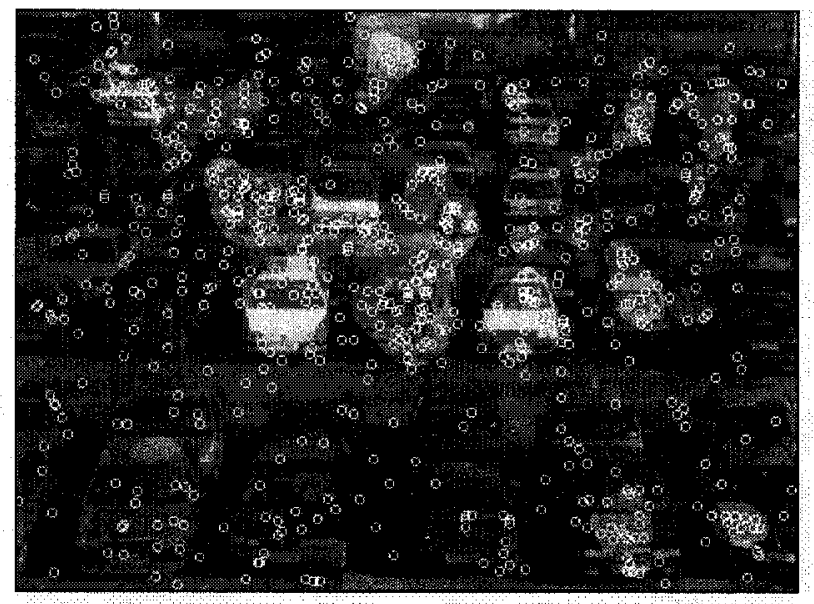

a) PP Cars Gaze

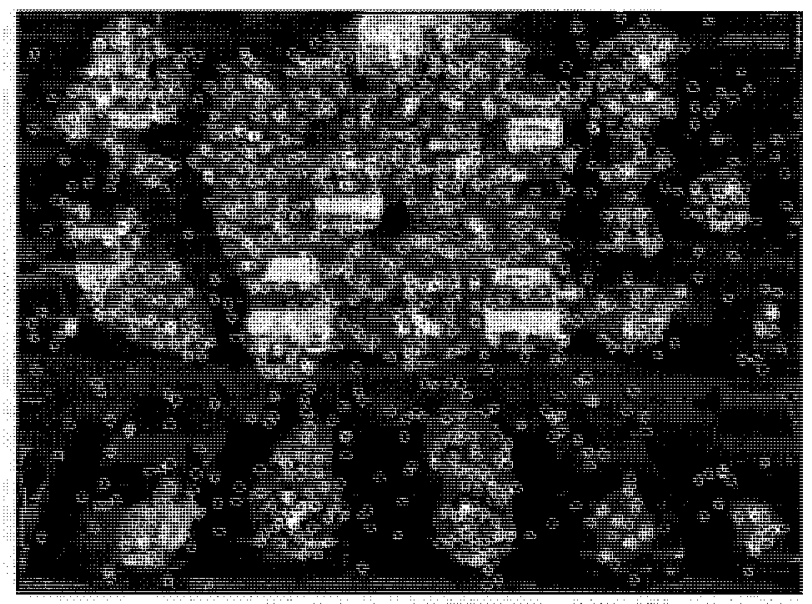

b) IR Cars Gaze

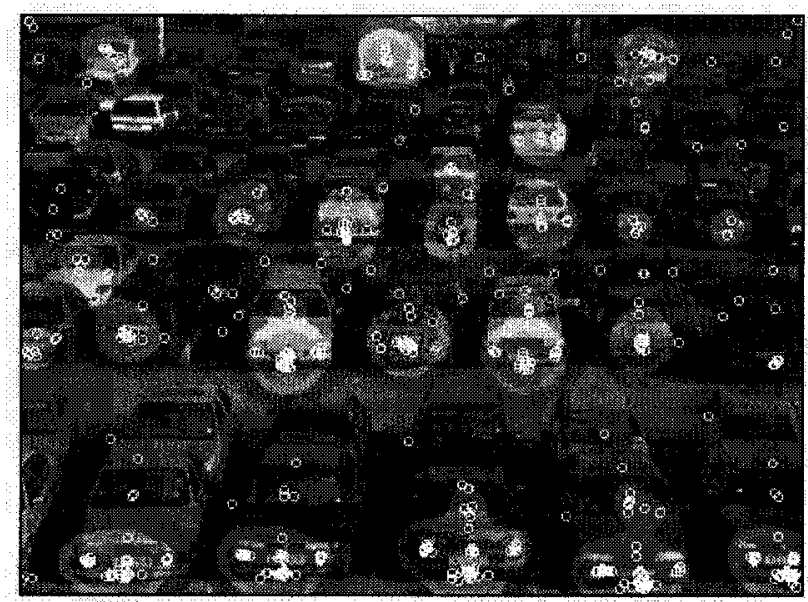

c) PP Field Click-points

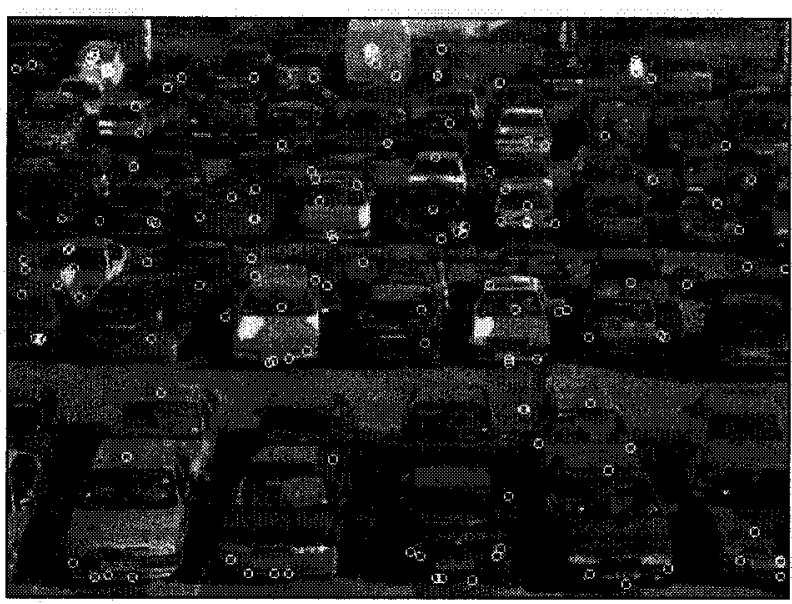

d) Old Lab Cars click-points

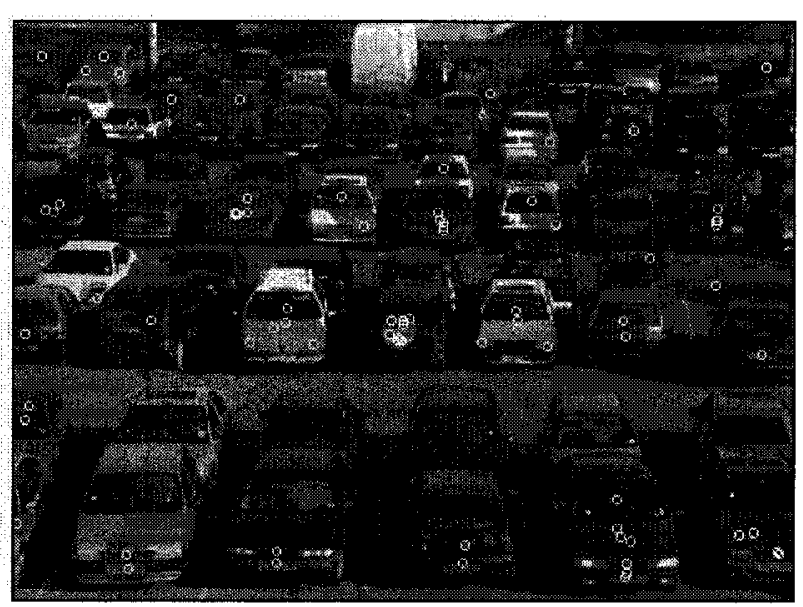

e) New Lab Cars Click-points

Figure 31. Summary images for the Cars image. a) PassPoints gaze fixations; b) Image Recall gaze fixations; c) PassPoints field study click-points data; d) Old lab study click-points data (Chiasson et. Al, 2007); e) Thesis lab study click-points data. 
The images in Figure 31 suggest that the best predictor of the field click-points data (represented by image c) would be the Image Recall (IR) gaze data (image b); this is the set of data points that seems to most accurately represent the click-points density found within the field image. The IR set of gaze data would best predict the old lab clickpoints data (image d) and the new lab click-points data (image e) when using a dictionary attack on all click-points data sets, as this is the data set that best overlaps the click-points data from the field study (image c).

In this image, participants tended to click on the license plates of the bottom rows of cars. However, looking at the gaze distribution data, participants very often looked at the van at the top of the screen, and also looked at the three people below the van. Surprisingly, not many people decided to click on the van; this could be due to the fact that participants believed that the van would be too great a target for others to guess their password, and thus dismissed it as a viable option. 


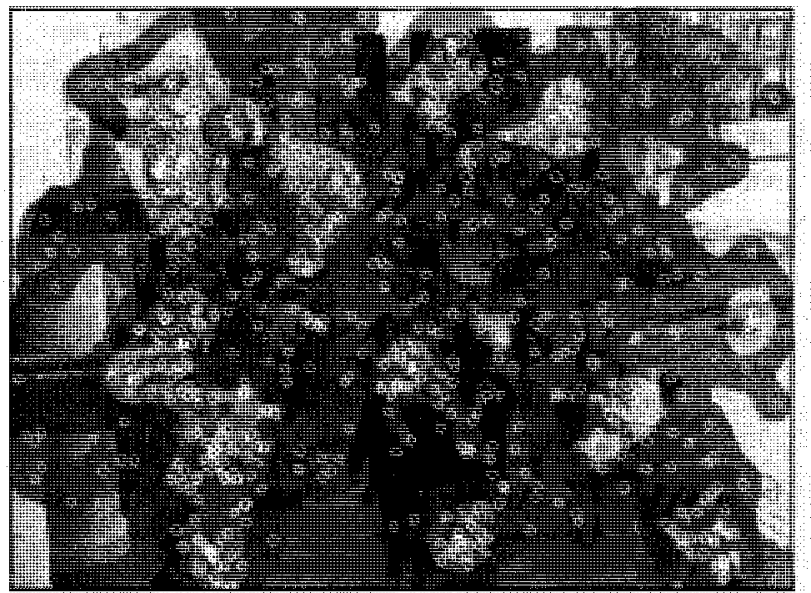

a) PP Pool Gaze

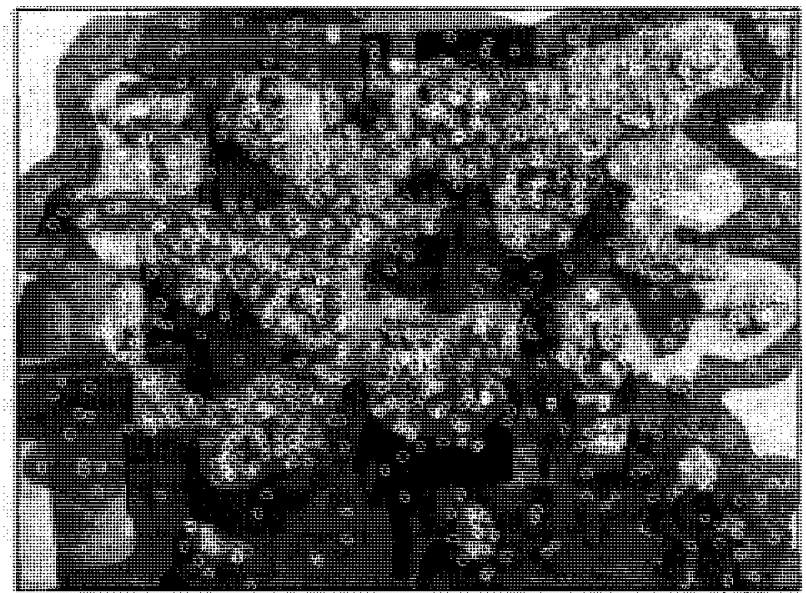

b) IR Pool Gaze

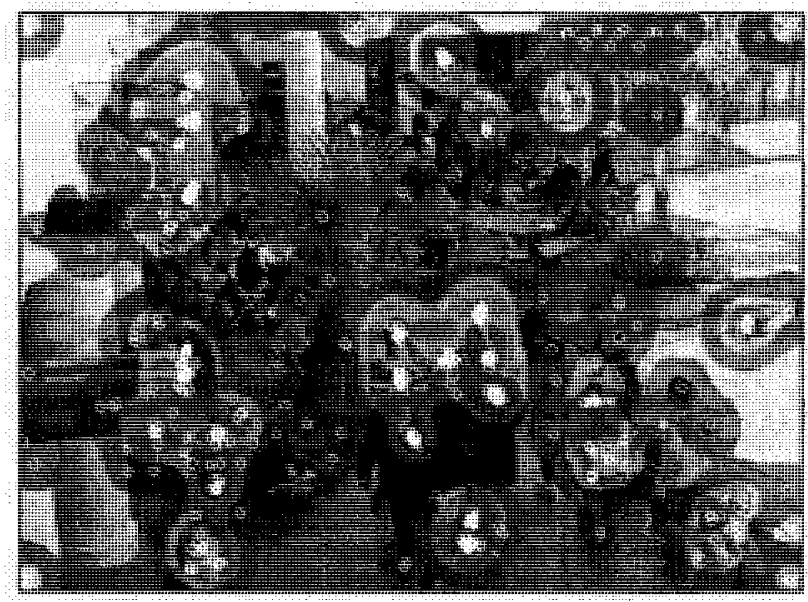

c) PP Field Pool Click points

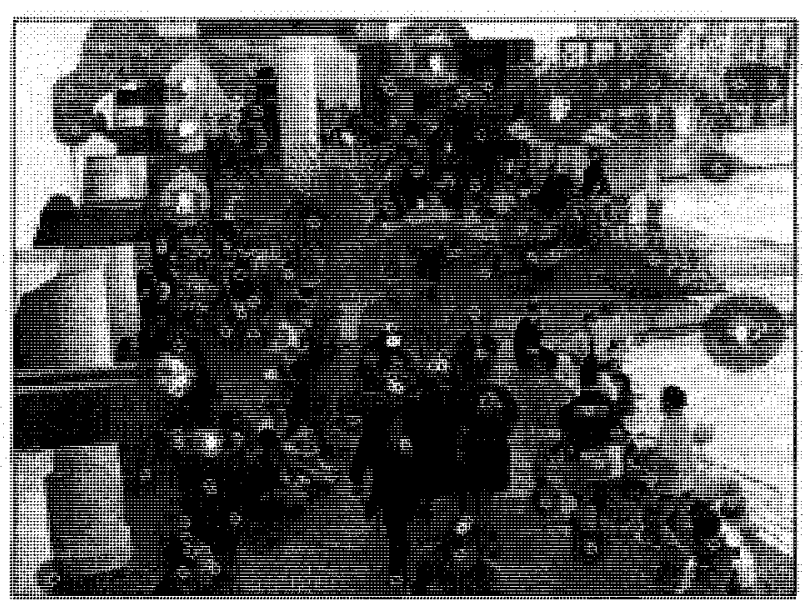

d) OId Lab Pool click-points

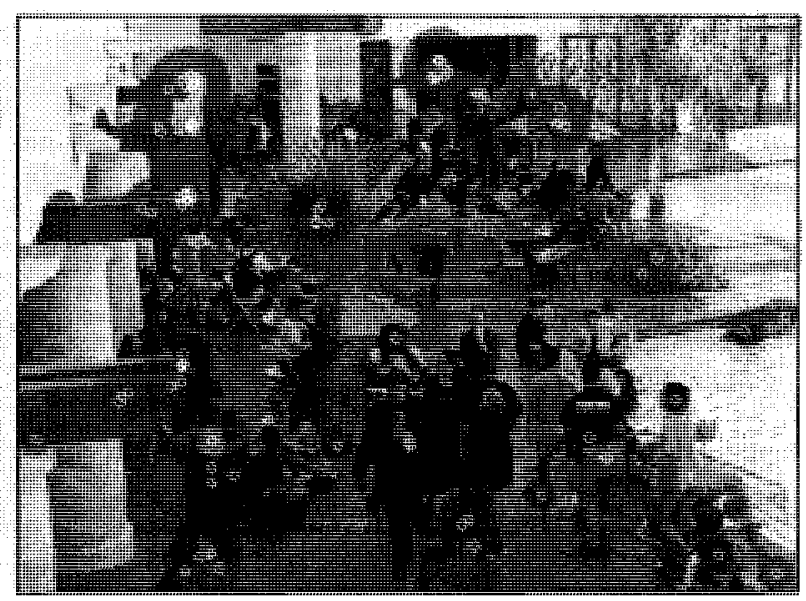

e) New Lab Pool Click-points

Figure 32. Summary images for the Pool image. a) PassPoints gaze fixations; b) Image Recall gaze fixations; c) PassPoints field study click-points data; d) Older lab study click-points data (Chiasson et al. (2007); e) Thesis lab study click-points data. 
The data set in Figure 32 shows us that the patterns are quite similar in both of the gaze images. Because these two patterns are so similar to one another, both the PassPoints gaze data and the Image Recall gaze data could potentially be used in order to attack the field click-points (image c). We can see that the Image Recall gaze data holds many more high density locations than does the PassPoints image, which one would assume would allow a better attack; however, many of those hotspots do not seem to overlap the field image, which would result in many missed guesses when performing the attack.

By looking at these images, it is apparent that the participants clicked on several of the same spots in the three studies (field study, old lab study, new lab study). Objects such as the metal beams on the left side of the image, the palm tree in the middle, the red garbage can, and so forth, were often used as targets when selecting click-points when creating graphical passwords. We can see by the gaze distribution data that participants looked at these same places, whether they wanted to click on it, or were simply attracted by the objects' features. 


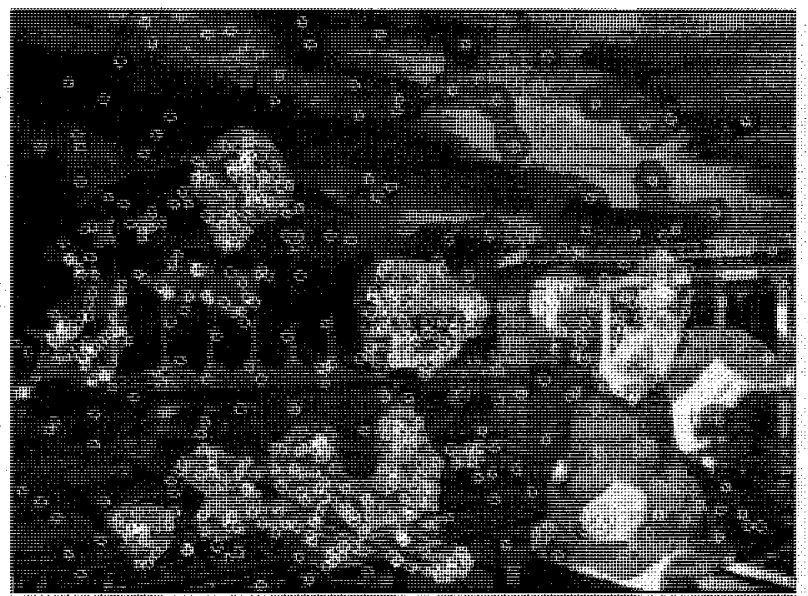

a) PP Statue Gaze

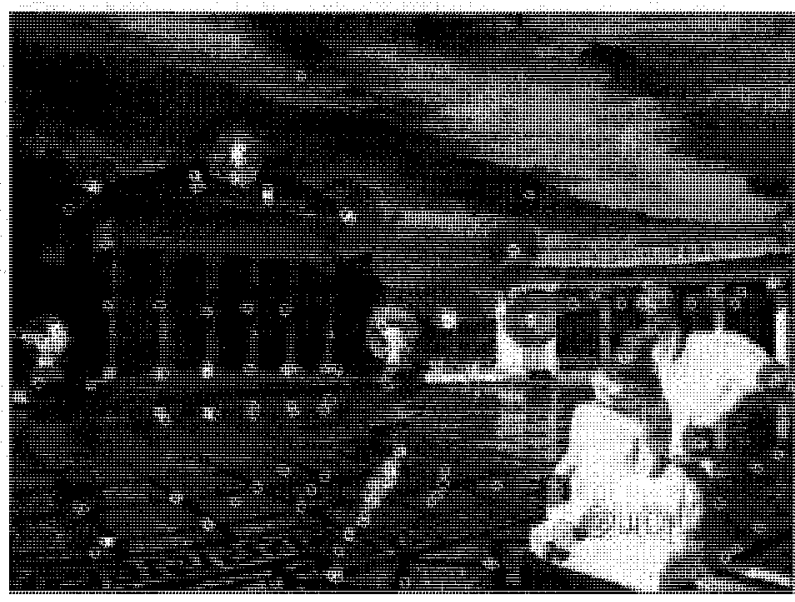

c) Old Lab Statue Click-points

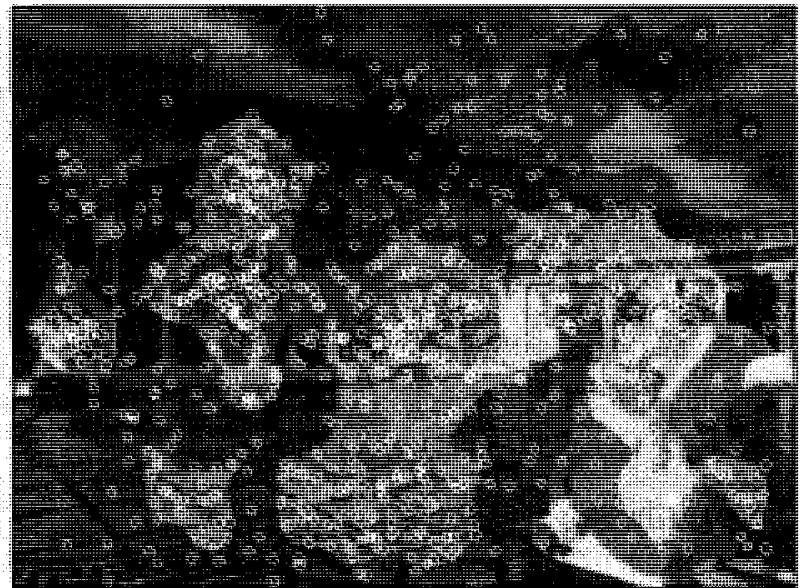

b) IR Statue Gaze

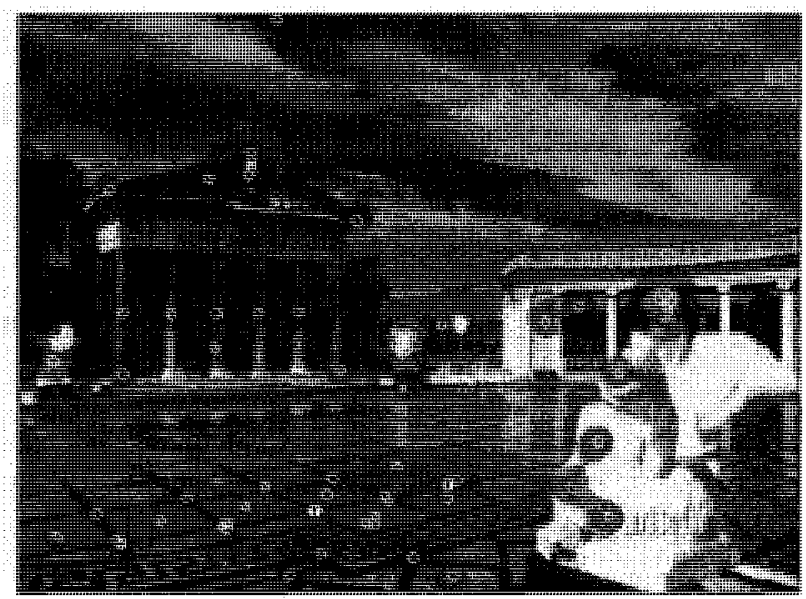

d) New Lab Statue click-points

Figure 33. Summary images for the Statue image. a) PassPoints gaze fixations; b) Image Recall gaze fixations; c) Older lab study click-points data (Chiasson et al. (2007); d) Thesis lab study click-points data.

As the rest of the images do not have field study data to be compared with, we will

compare the PassPoints and Image Recall gaze data images ( $a$ and $b$ ) only with the old and new PassPoints graphical password studies (images $\mathrm{c}$ and d). As seen in Figure 33, it would seem most logical to attack the old lab study click-points (image c) as well as the new lab study click-points, with the PassPoints gaze data (image a). This set of gaze data seems to share more of the hotspots in common with the two lab studies. 
In this particular image, we can see that most participants opted to click either on the bottom of the pool, or on the columns or statues of the buildings in the background. However, the gaze distribution data suggests that participants looked at several other regions on the image as well as places they clicked.

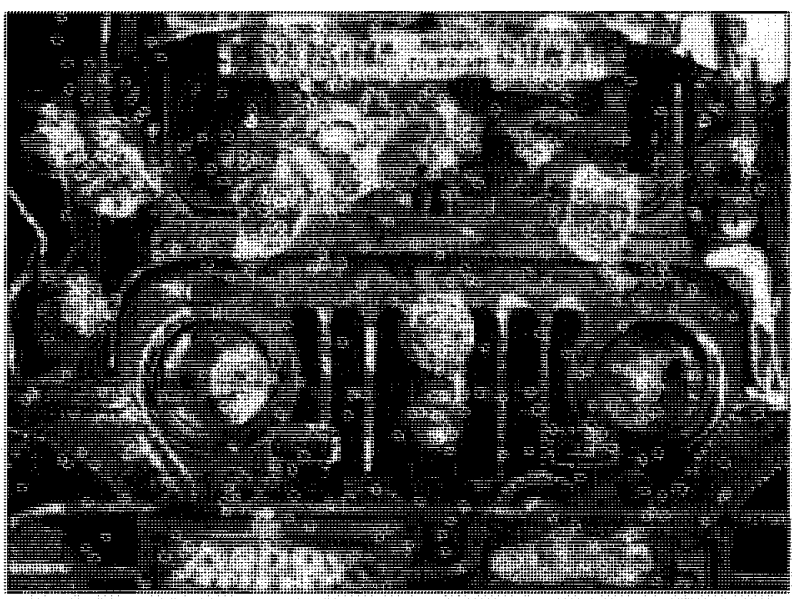

a) PP Truck Gaze

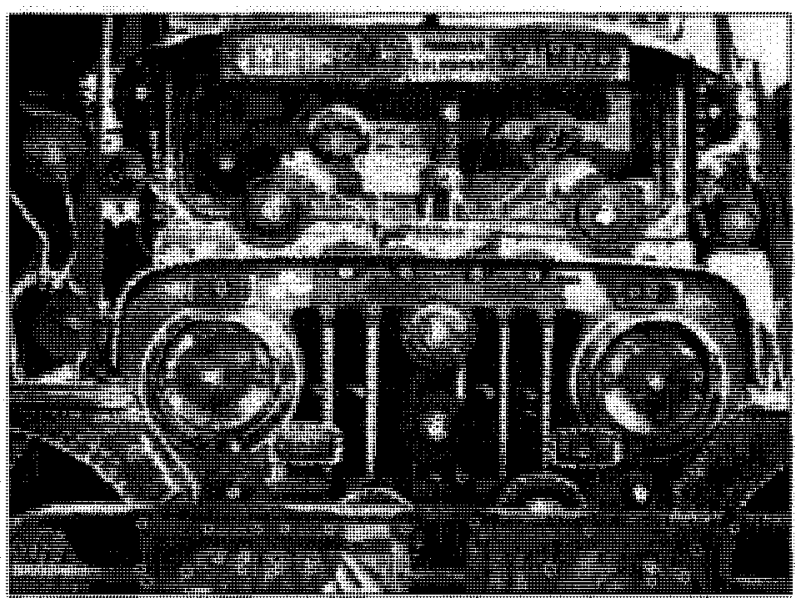

c) Old Lab Truck Click-points

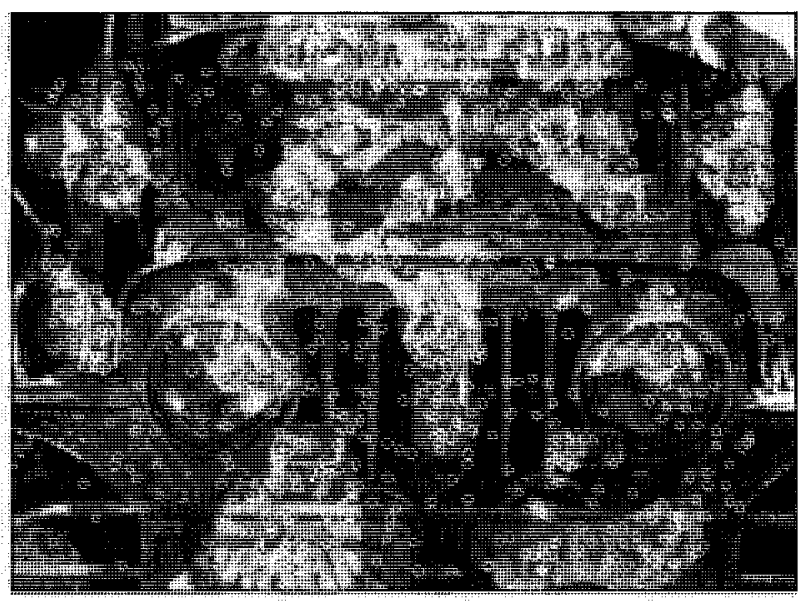

b) IR Truck Gaze

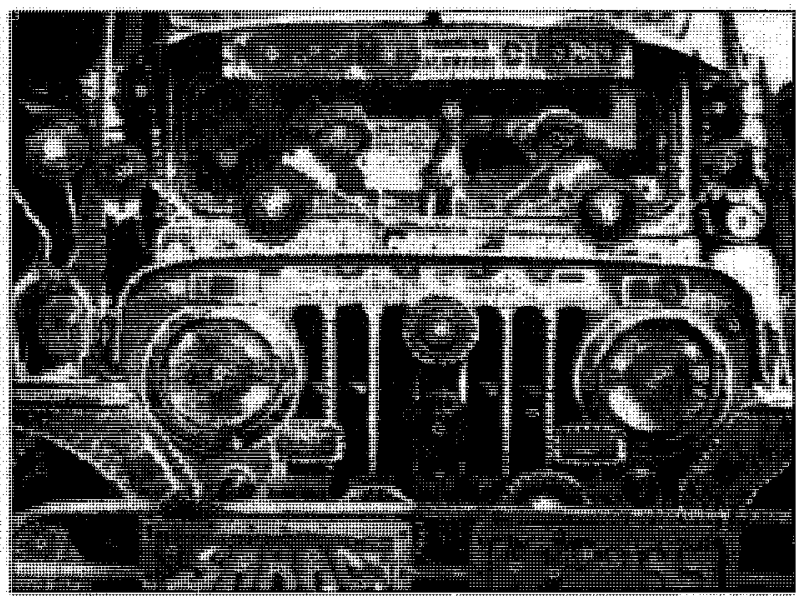

d) New Lab Truck Click-points

Figure 34. Summary images for the Truck image. a) PassPoints gaze fixations; b) Image Recall gaze fixations; c) Older lab study click-points data (Chiasson et al. (2007); d) Thesis lab study click-points data.

For this particular set of data, as seen in Figure 34, it would seem apparent that the Image

Recall gaze data (image b) would best predict the click-points found in either the old lab 
study (image c) or the new lab study (image d). In particular, the hotspots of interest are the two license plates at the bottom of the truck image, the sign at the front and on top of the jeep's windshield, and the two headlamps; these seem to be better represented by the hotspots found in the Image Recall gaze data.

This particular image is quite special in terms of its apparent explosion of colours, objects, and patterns. Participants were attracted to the license plates at the bottom of the image, the wording on the top of the image, and the headlights of the jeep, regardless of the condition in which the participant was assigned to (i.e. the PassPoints or Image Recall conditions). Whether they were looking or selecting click-points, the most popular hotspots for gaze or click data was on or around those areas. 


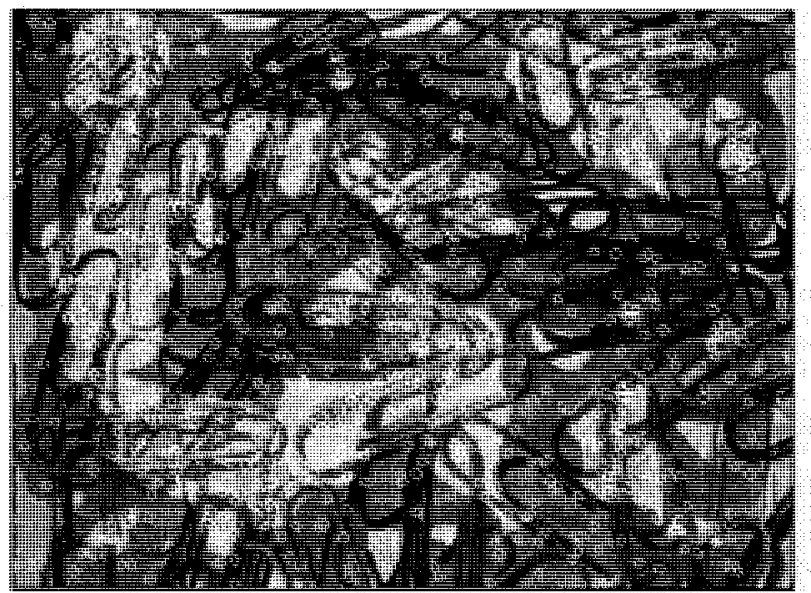

a) PP Paperclips Gaze

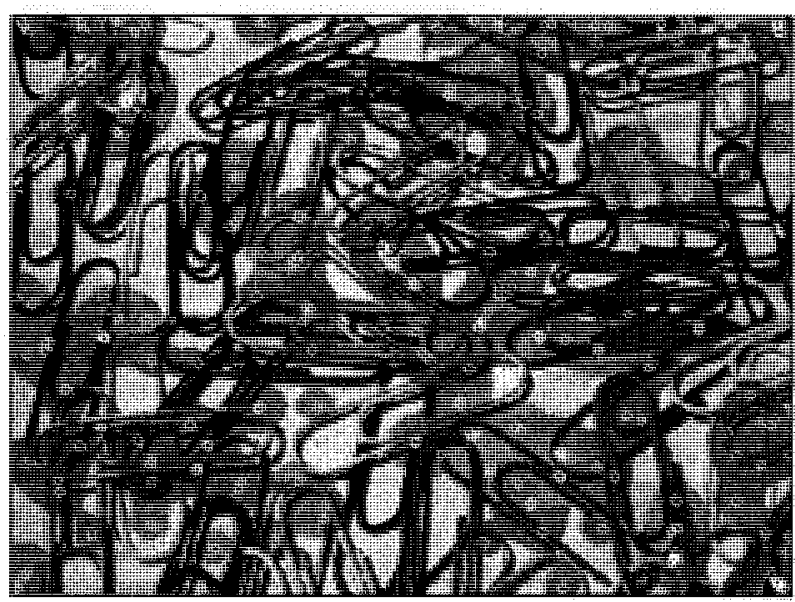

c) Old Lab Paperclips click-points

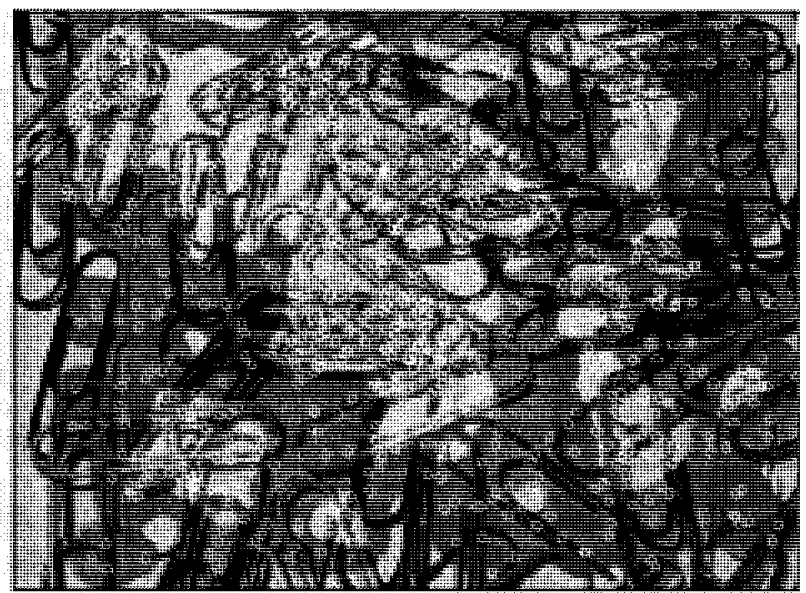

b) IR Paperclips Gaze

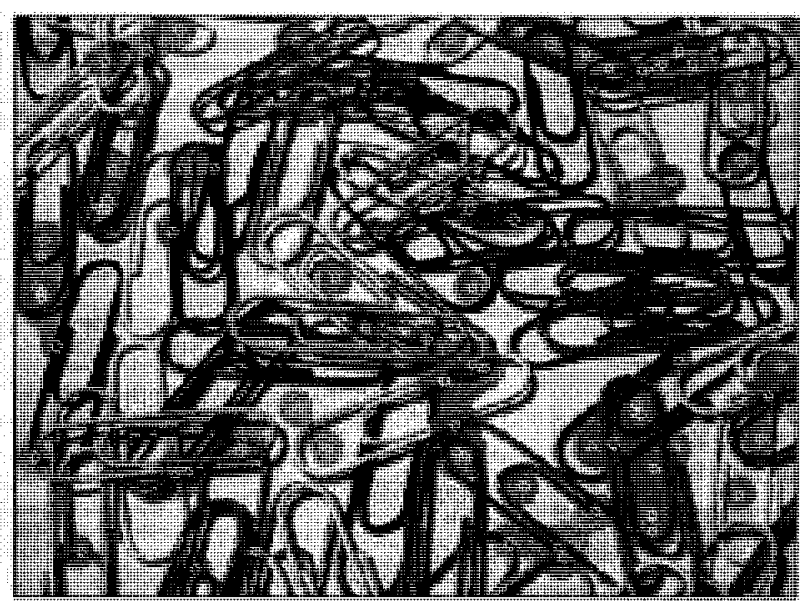

d) New Lab Paperclips Click-points

Figure 35. Summary images for the Paperclips image. a) PassPoints gaze fixations; b) Image Recall gaze fixations; c) Older lab study click-points data (Chiasson et al. (2007); d) Thesis lab study clickpoints data.

In Figure 35, we see that the data points found in the old lab study data (image c) are more evenly spread out, unlike the click-points found in the new lab study data. As such, it would seem that the most logical choice of action would be to attack the old lab study data with the wider-reaching Image Recall task gaze data set (image b). However, the new lab click-point data seems to better fit the patterns found in the PassPoints gaze data set (image a). 
This image was one of the more difficult for participants to adapt to, whether they were selecting click-points in order to create a graphical password, or were simply staring at the image for the Image Recall task. We can see that the patterns for all conditions do not overlap very well. This could be due to the fact that the objects in the image appear to have been thrown in together with no salient details.

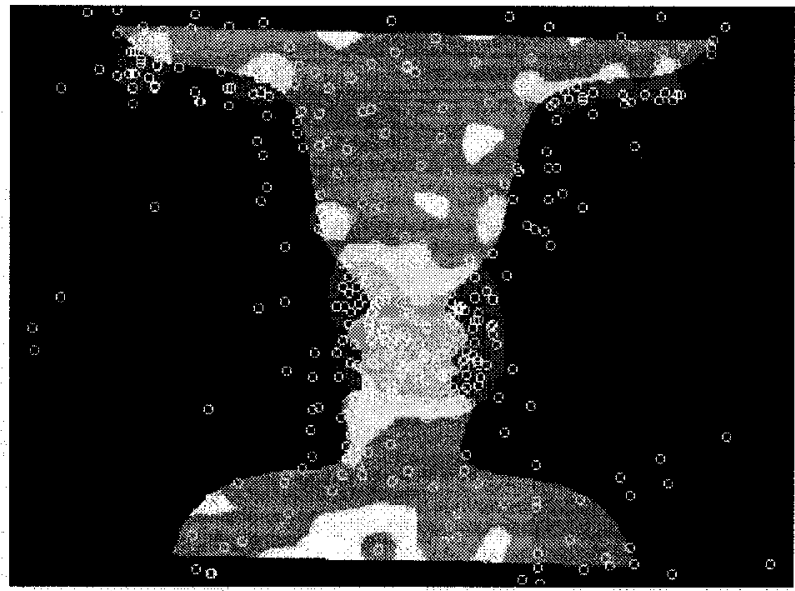

a) PP Figureground Gaze

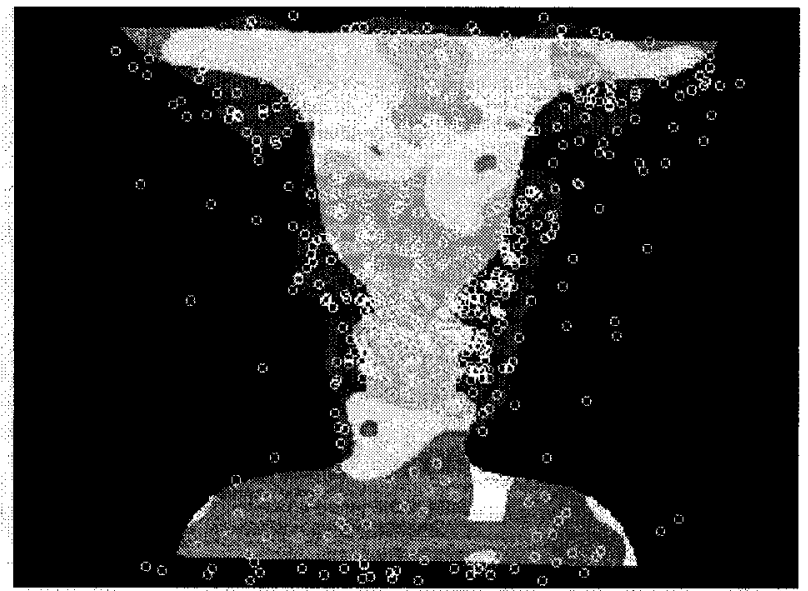

b) IR Figureground Gaze

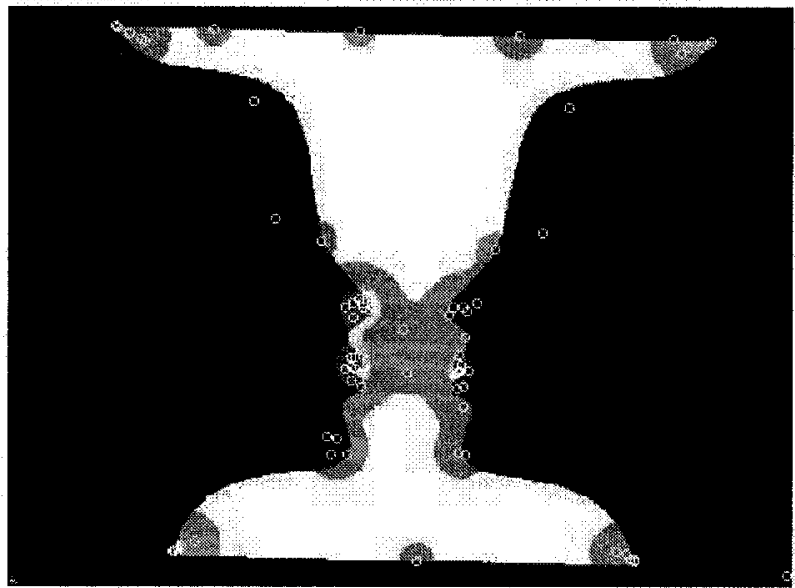

c) New Lab Figureground Click-points

Figure 36. Summary images for the Figureground image. a) PassPoints gaze fixations; b) Image Recall gaze fixations; c) Thesis lab study click-points data.

For the remaining 3 images, we only have click-points data for the new lab study; as such, we will decide which of the two gaze data points seems more apt for use in a 
dictionary attack. As we can see in Figure 36, the patterns chosen by the participants seem to concentrate in a few specific places; therefore, most participants selected very similar point patterns (i.e. clicking the nose, eyes, lips, chins, and the top and bottom corners). In this case, it should not matter which gaze data is used, as both the PassPoints and the Image Recall gaze distributions encompass each of the hotspots found in the new lab click-points data.

As this image is not as complex as some of the others (the Paperclips image, as an example), participants chose to select click-points that were based on the outlines of the figures. It was in this same manner that the participants in the Image Recall condition gazed at the image. 


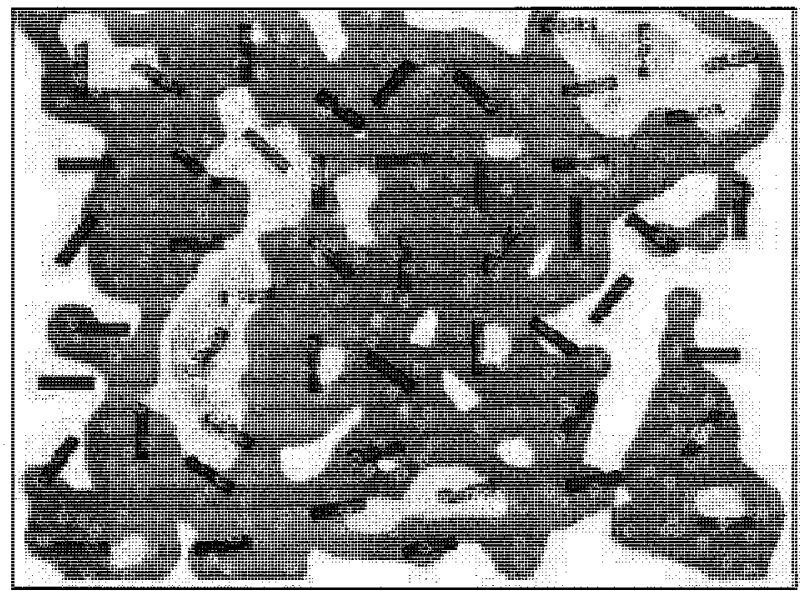

a) PP Topdown Gaze

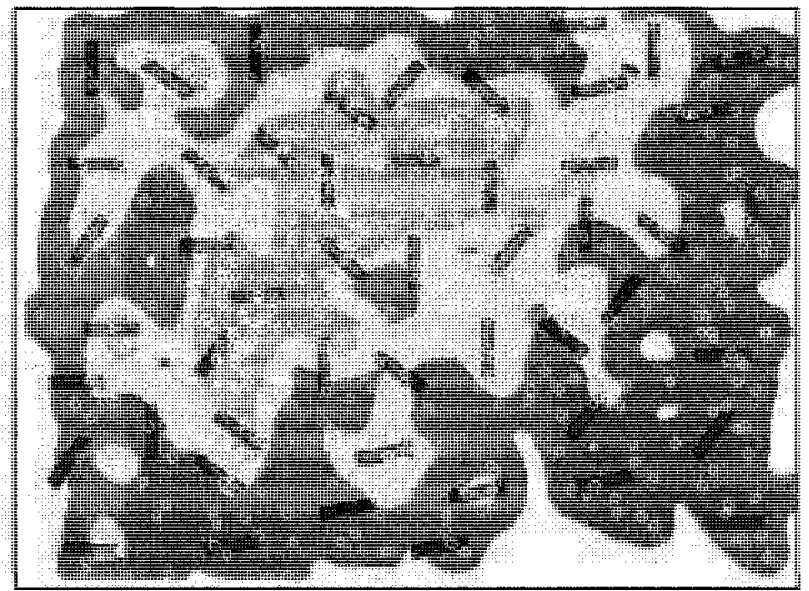

b) IR Topdown Gaze

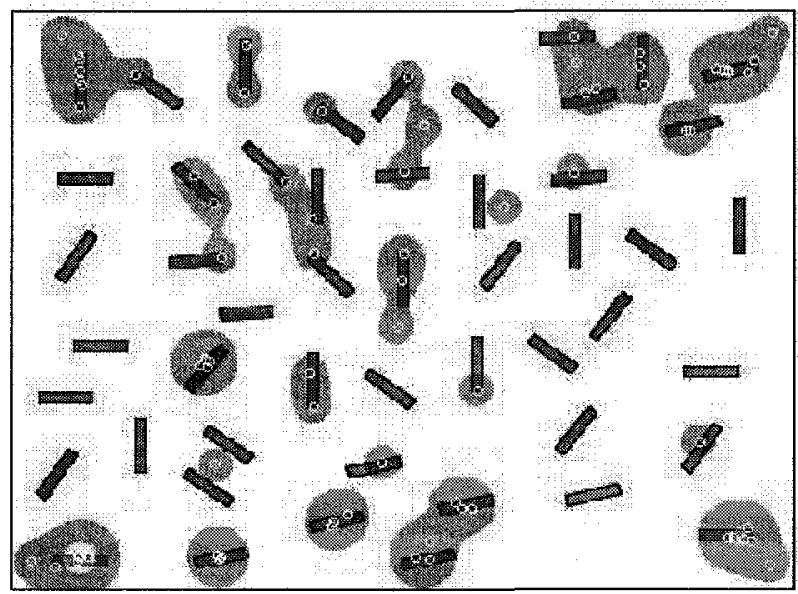

c) New Lab Topdown Cliek-points

Figure 37. Summary images for the Topdown image. a) PassPoints gaze fixations; b) Image Recall gaze fixations; c) Thesis lab study click-points data.

In Figure 37, we can see that there is very little clustering of the gaze points in the PassPoints gaze image (a). As such, it would seem logical to use the Image Recall gaze distribution (image b) in order to attack the new lab study click-points (image c).

Participants frequently selected the red rectangle which stood out in this image. Upon having made this selection, they quickly began to map out their own patterns, either by selecting left to right patterns of green rectangles, or top to bottom, or even the four corners and the middle of the image. Since the patterns in this image were complex, the 
gaze distribution data for either condition does not reflect well the click-points selected by the participants in the old lab study.

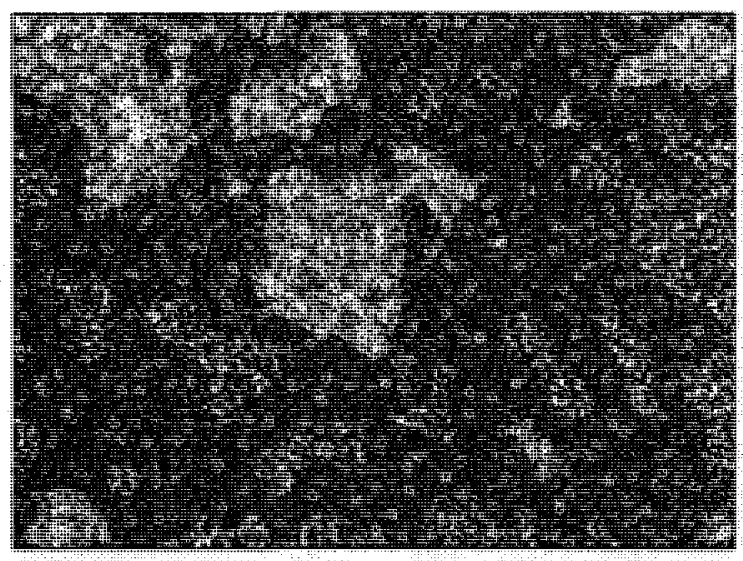

a) PP Topdown Gaze

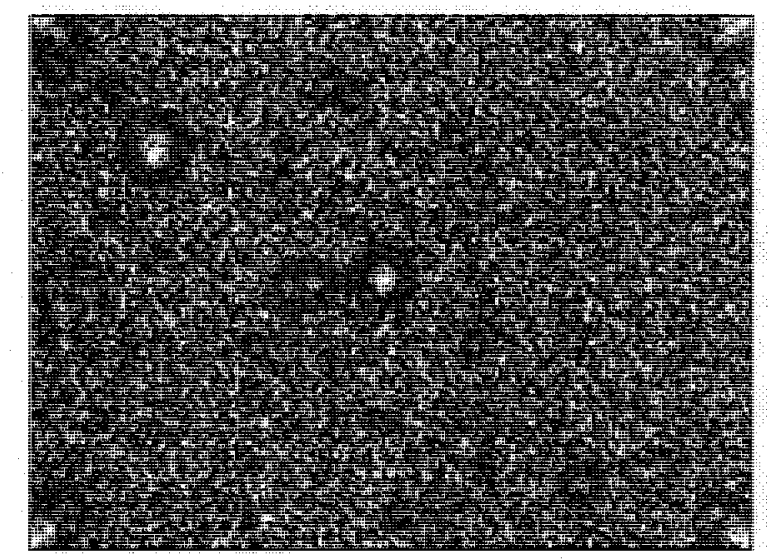

c) New Lab Topdown click-points

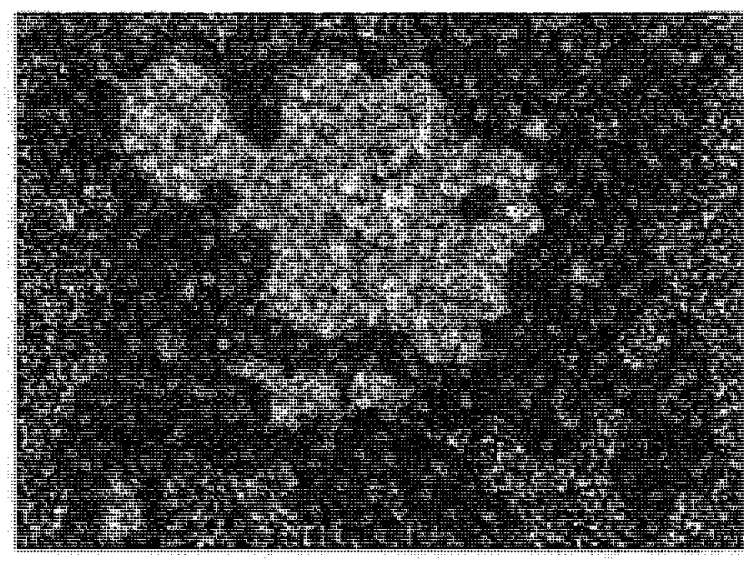

b) IR Topdown Gaze

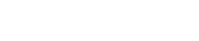


Due to the lack of patterns and objects in this image, participants opted to click on the four corners of the image, as well as the center. This does not bode well for the security of the image as one to use as a graphical password image. Gaze patterns reflect that participants, upon having inspected the image for a few seconds, gazed mostly towards the center of it for the remaining time left in the trial.

\section{Hypothesis 3 Summary: Image Analysis for New Clicks and Gaze Data}

We first compare all eight images in this section, which were part of the new lab study. The new lab study click-points for each of these images represent smaller numbers than the old lab study click-point data. From the heatmaps associated with each image, we can see that, even though the Image Recall condition gaze distributions comprise more individual fixations than does the PassPoints condition, the latter more often offers a better pattern match to the new click data. Some of the patterns showed potential for matching one to the other, including the Pool, Cars, Truck, Statue, and Figureground images, but they did not show this same potential for the Paperclips, Topdown, and Noise images.

Hypothesis 3 Summary: Image Analysis for Old Clicks, Field Clicks, and Gaze Data

Contrary to the eight images used in the new lab study, only five images in our study had old lab click-points data, and two of these had the field click-points data; these were the Pool(with field), Cars(with field), Truck, Statue, and Paperclips images. For the majority of these images, the Image Recall gaze distribution data more closely resembles their click-point patterns. Table 17 depicts the comparisons between the new clicks and 
the old clicks, as well as the gaze distribution data for both the PassPoints and Image Recall conditions. It does seem that the gaze distribution data for both the PassPoints and the Image Recall conditions overlap the new and old lab study click-points suggests that our hypothesis can potentially be justified from this analysis of the images. The possible exception appears to be the Paperclips image.

Table 17. Comparison of analytical results for the Image Analysis section of Hypothesis 3.

\section{Comparison of Analytical Results}

Heatmap Analysis

\begin{tabular}{llll} 
Target: & New Clicks & Old Clicks & \\
Best Attack: & Of Gaze Heatmaps & Of Gaze Heatmaps & Overall \\
\hline Cars & IR & IR & Similar \\
Pool & PP & PP & Similar \\
Statue & PP & IR & Clicks \\
Truck & IR & IR & Similar \\
PaperClips & PP & IR & Clicks \\
TopDown & Similar & $*$ & $*$ \\
FigureGround & IR & $*$ & $*$ \\
\hline
\end{tabular}

Attack Dictionary Analysis for Hypothesis 3

In this section, we explore hypothesis 3 by creating attack dictionaries following the method of Thorpe and van Oorschot (2007). In the analysis below, we use the term dictionary attack to describe our guessing of individual click-points. For security analysis, we acknowledge that we would need to examine whole passwords; but, in this study, we focused on the relationship between gaze fixations and click-points, leaving whole passwords for later work. We would anticipate that the security of passwords would be affected by the number of gaze fixation points that overlap click-points, even if in most passwords had one or two click-points that did not. 
We created attack dictionaries based on three distinct groups: new clicks hotspots, PassPoints gaze hotspots, and Image Recall gaze hotspots. Each of the attack dictionaries was created as an ordered list of points following the method described at the beginning of section 3.1 in the paper by Thorpe et al. (2007). This method consisted of ranking each point in the data set by the number of neighboring points (within the tolerance region of 19 by 19 pixels), and eliminating duplicates. This gave us dictionaries consisting of points in order of possible likelihood of selection by users. In the analysis that follows, we showed the effectiveness of guessing based on these dictionaries using an increasing number of the dictionary click-points. For the gaze data, we also took into consideration the duration of each fixation, counting every 100 milliseconds of fixation as an additional point for the purposes of ranking the click-points.

We assessed the effectiveness of each attack dictionary by taking the points in the dictionary in order, and determining the cumulative number of points within tolerance from the target set of click-points we are attacking. For example, we took the highest ranking point in the dictionary, and determined how many user click-points were within tolerance of that dictionary point. We then took the top 2 highest ranking points in the dictionary, and so on. In the graphs that follow, we show the effectiveness of the attacks for each image. The targets of the attacks are the click-points chosen in graphical passwords from our study, as well as the earlier lab studies conducted by Chiasson et al. (2007) and, for the Cars and Pool images, their larger field study. In Figures 39 through 53 , the $\mathrm{X}$ axis shows the number of ranked click-points used from the dictionary, and the $\mathrm{Y}$ axis shows the cumulative ratio or proportion of hits, which is the fraction of the total number of click-points that were guessed. Some of the lines on the graph do not reach the 
right side of the graph, because of the limited size of the data sets. On each of the graphs, we also show a straight line with a slope that indicates the proportion of passwords that we would expect to guess by chance. With a tolerance region of 19 by 19 , the image consists of 414 distinct places to click and each chance guess would expect to find $1 / 414$ of the click-points.

\section{Dictionary attack on the new lab study data (Cars)}

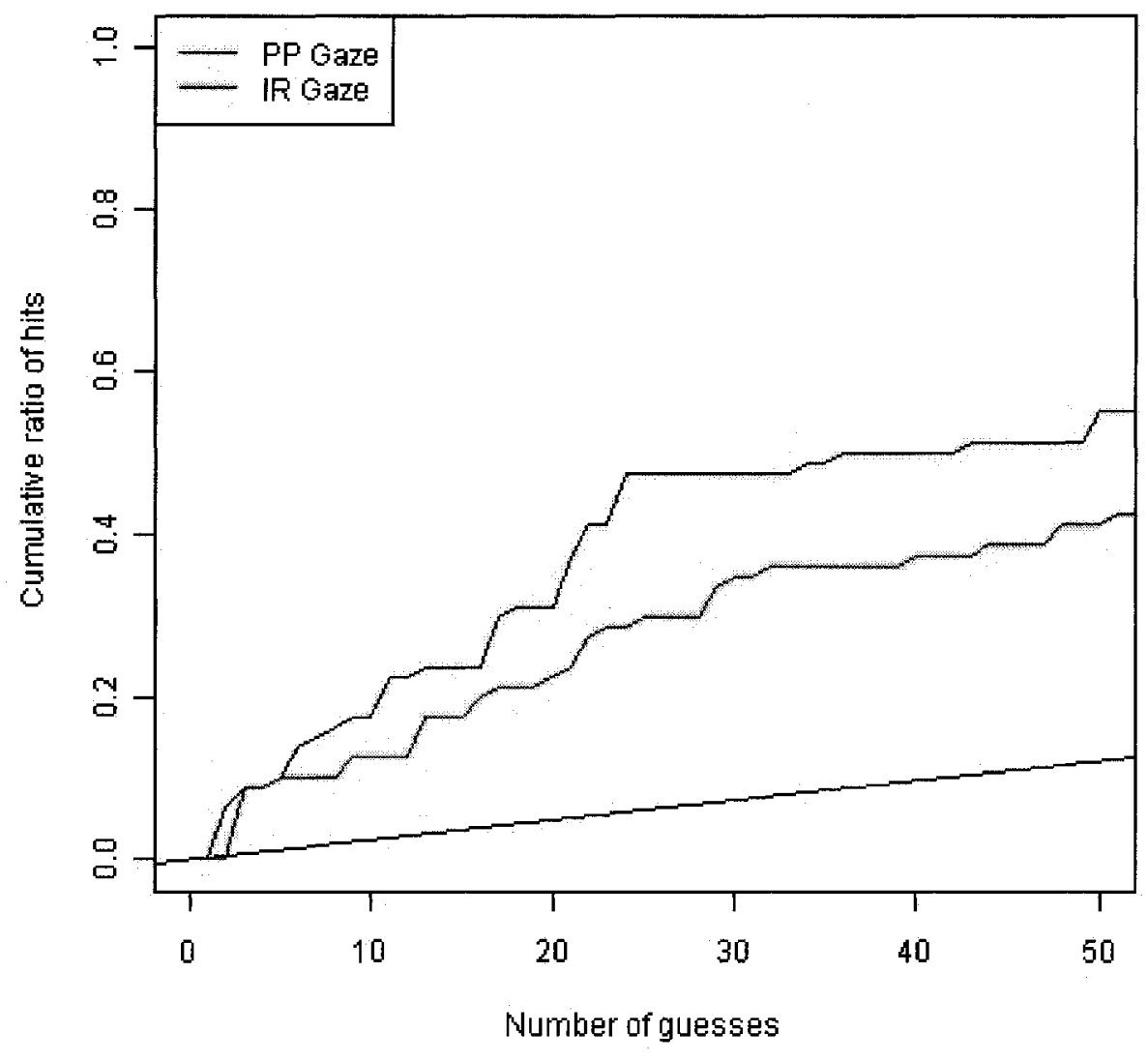

Figure 39. Dictionary attack on the new lab study of the Cars image, plotted as the cumulative ratio of hits as a function of the number of guesses.

The attacks on the Cars image, this time using the PP gaze data as well as the IR gaze data to attack the new clicks data, demonstrates that there is a lot of similarities between 
the two data sets, as they do not stray very far apart from each other on the cumulative hit ratios, as the number of guesses increases.

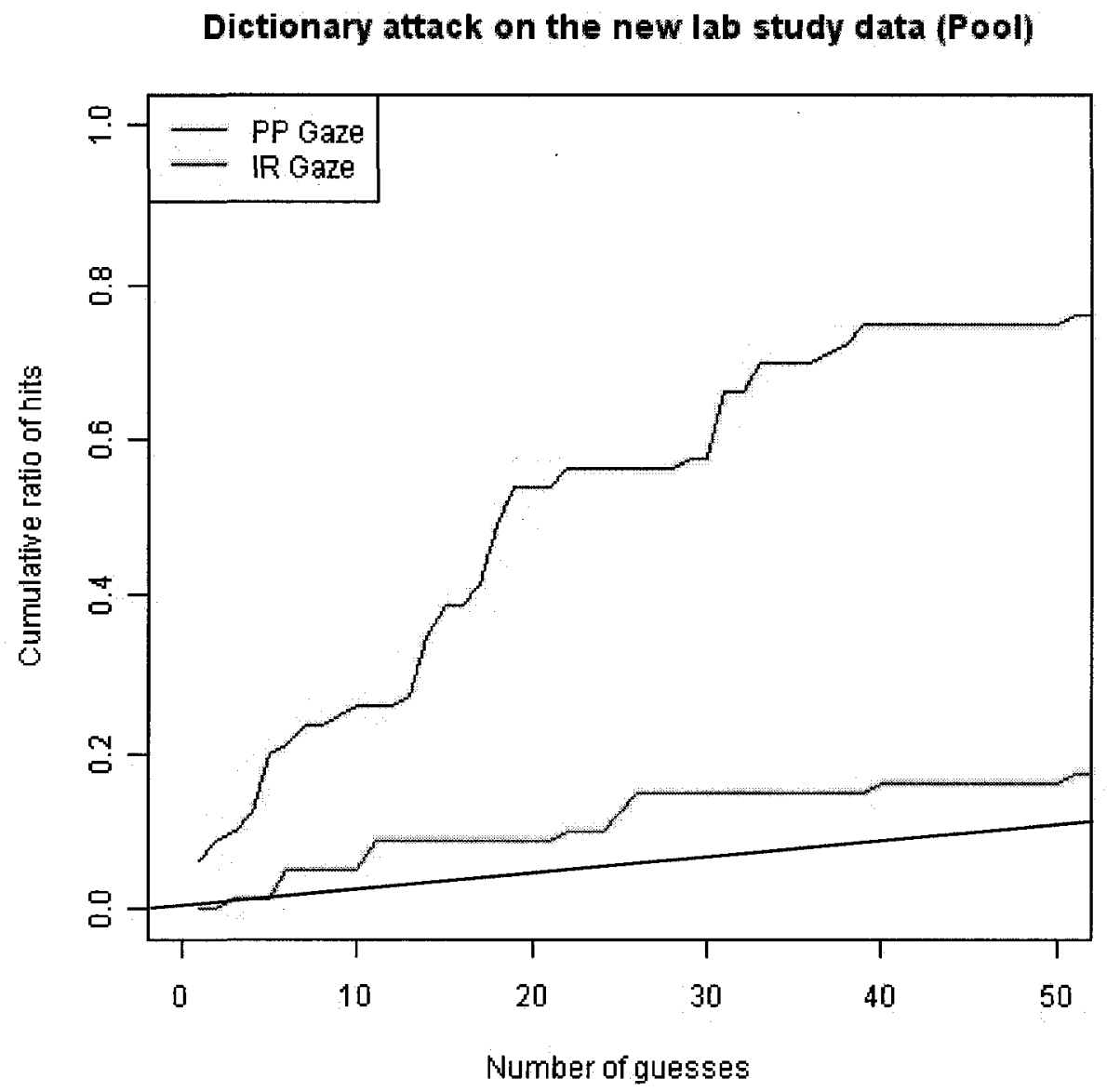

Figure 40. Dictionary attack on the new lab study of the Pool image, plotted as the cumulative ratio of hits as a function of the number of guesses.

In Figure 40, we're attacking the new lab click-points data with the PP gaze and IR gaze data distributions. We can see by the graph that the PP gaze distribution guesses more than double the correct number of click-points within 10 guesses, and correctly guesses approximately $80 \%$ of all click-points after 50 guesses, compared to the IR gaze data which guesses only about $16 \%$ of the click-points after the same number of guesses. 
Dictionary attack on the new lab study data (Truck)

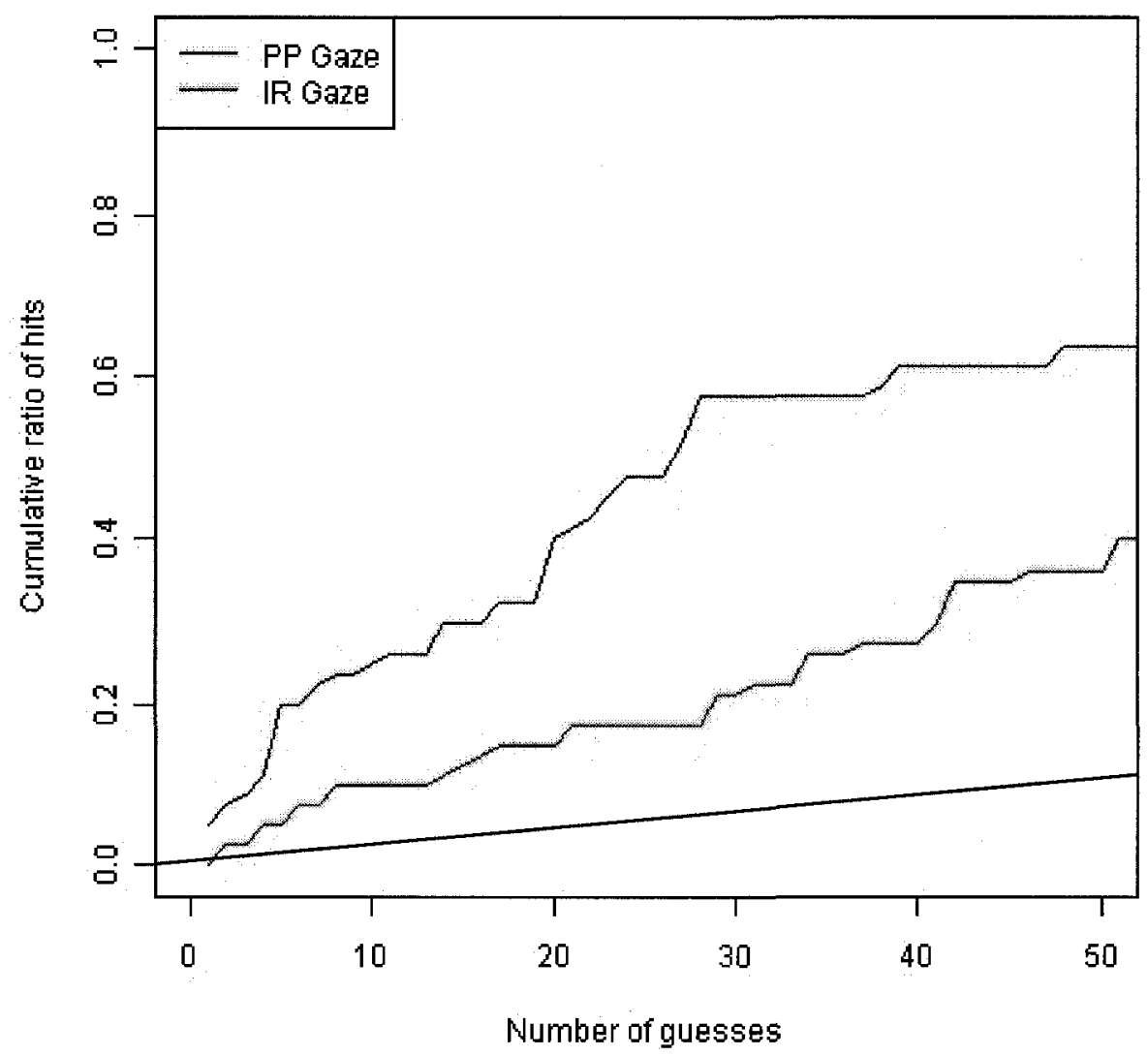

Figure 41. Dictionary attack on the new lab study of the Truck image, plotted as the cumulative ratio of hits as a function of the number of guesses.

Figure 41 shows us that the PP gaze data is slightly better than the IR gaze data at predicting the correct click-points of the new lab study data. The largest difference in the number of correct guesses occurs at approximately the $28^{\text {th }}$ guess. 


\section{Dictionary attack on the new lab study data (Paperclips)}

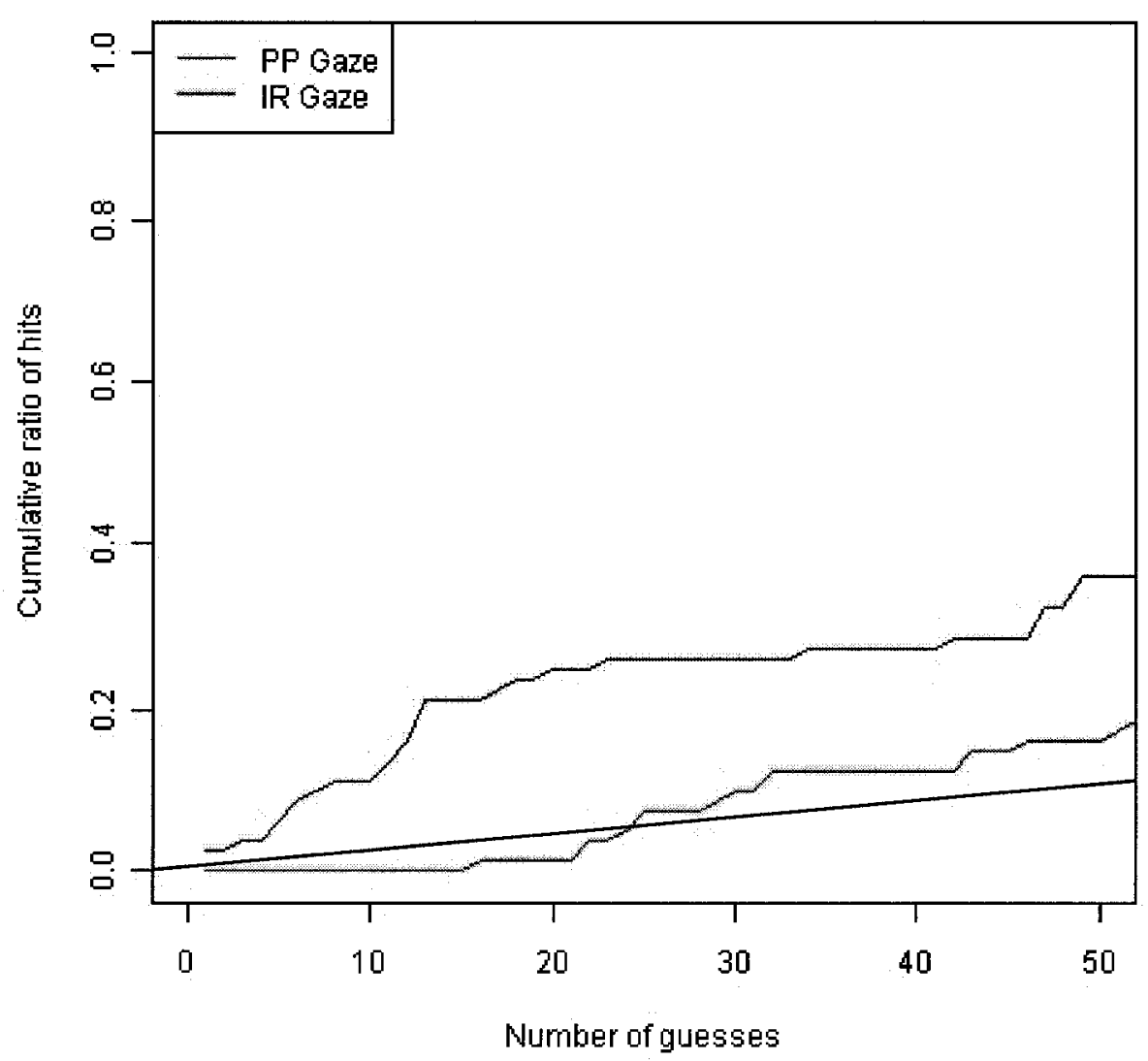

Figure 42. Dictionary attack on the new lab study of the Paperclips image, plotted as the cumulative ratio of hits as a function of the number of guesses.

For this dictionary attack on the Paperclips image, the PP gaze data distribution is slightly better than the IR gaze data at predicting the new lab study click-points. 
Dictionary attack on the new lab study data (Statue)

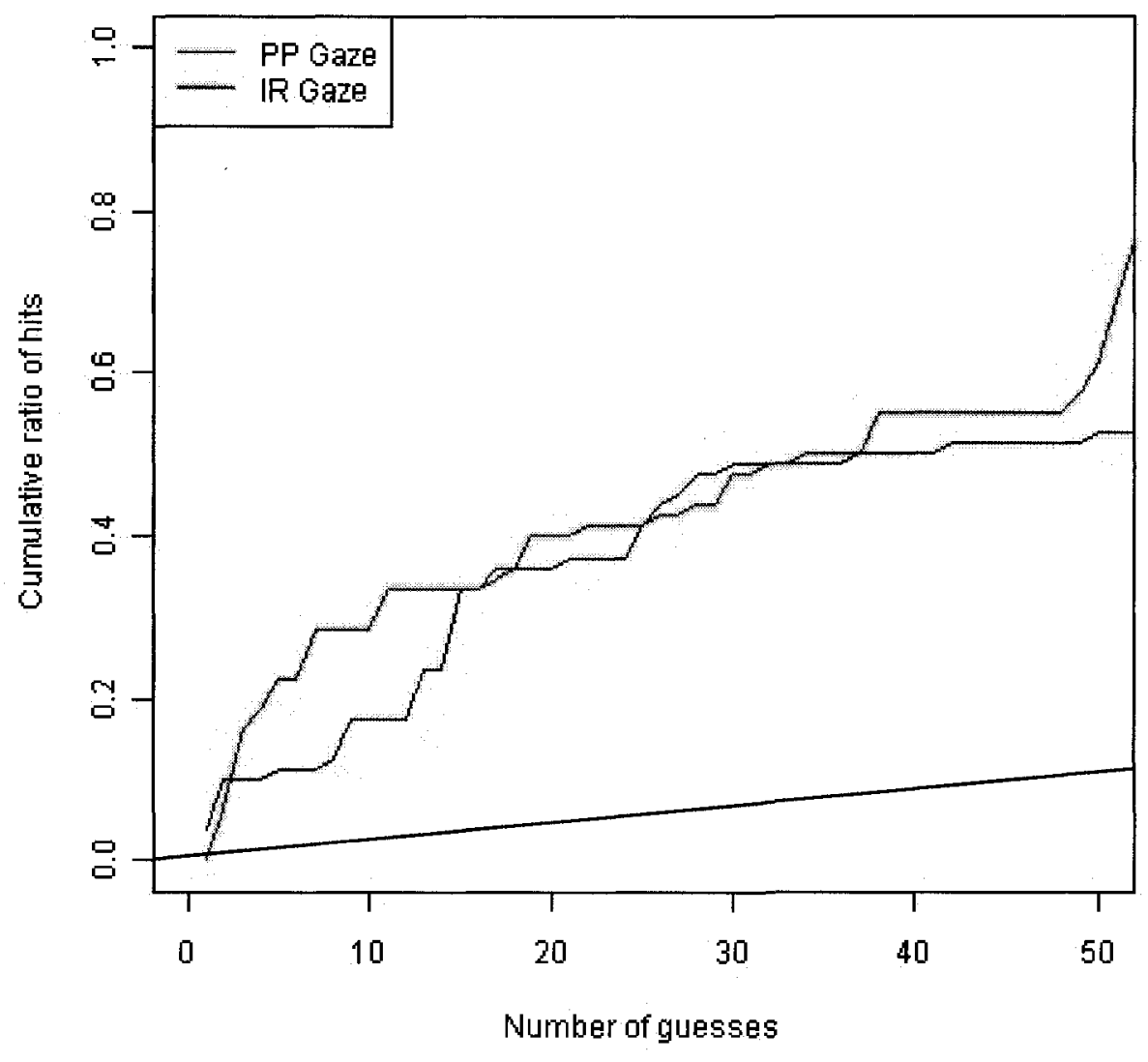

Figure 43. Dictionary attack on the new lab study of the Statue image, plotted as the cumulative ratio of hits as a function of the number of guesses.

This particular dictionary attack on the Statue image is of particular interest, as it is the only one that follows such a unique intertwining pattern between the PP gaze data and the IR gaze data. After the $15^{\text {th }}$ guess, both gaze distributions correctly guess approximately the same number of click-points from the new lab study data; however, after 50 guesses, there is a sharp rise in the correct number of guesses for the IR gaze distribution data, where there is minimal change in the number of correct guesses for the PP gaze data. 
Dictionary attack on the new lab study data (Figureground)

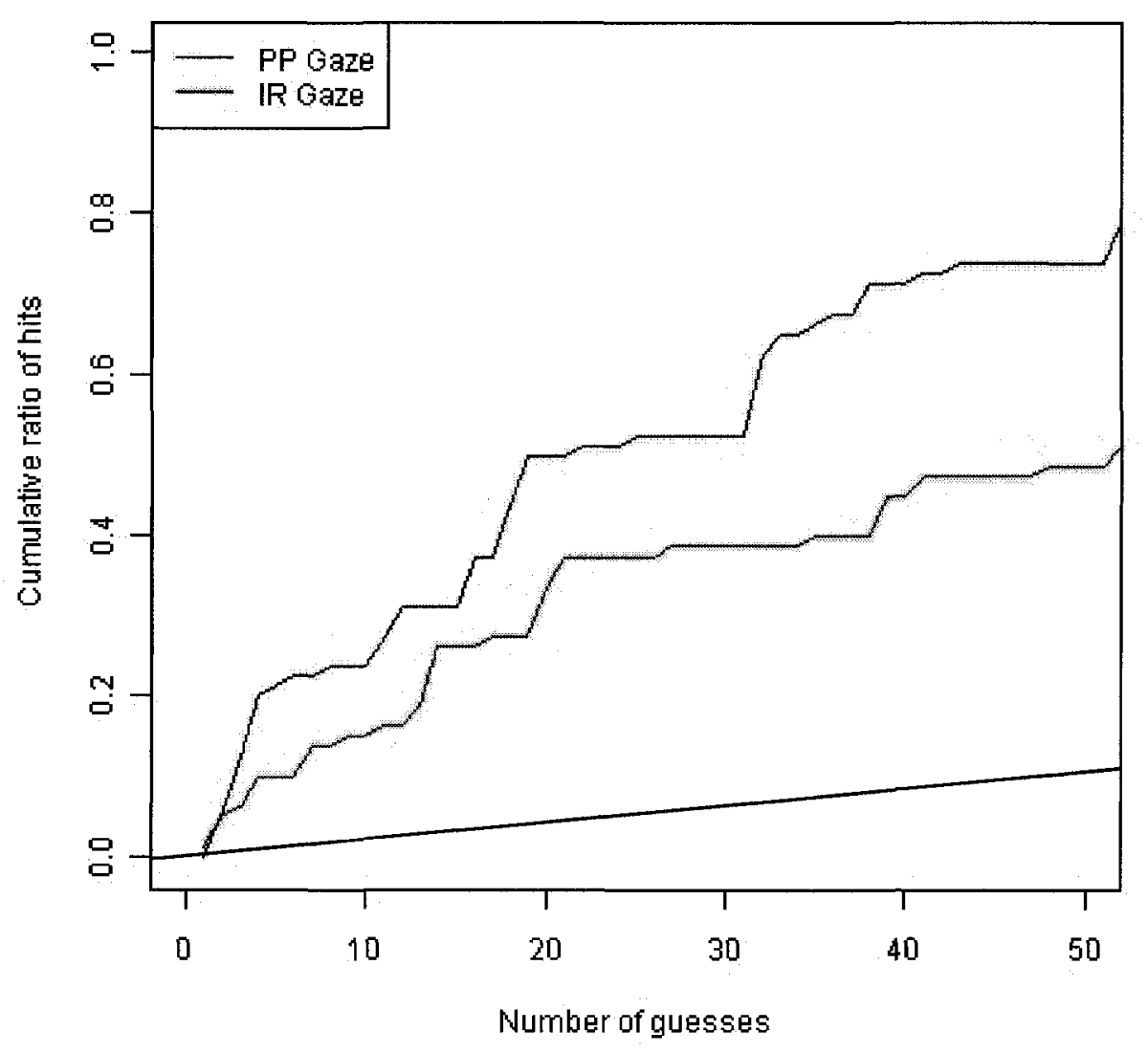

Figure 44. Dictionary attack on the new lab study of the Figureground image, plotted as the cumulative ratio of hits as a function of the number of guesses.

In Figure 44, we see a minimal difference in correct guesses on the new lab study data, until we come to approximately the $30^{\text {th }}$ guess, where there is a sharper increase of the correct number of click-points guessed for the PP gaze distribution data. 
Dictionary attack on the new lab study data (Topdown)

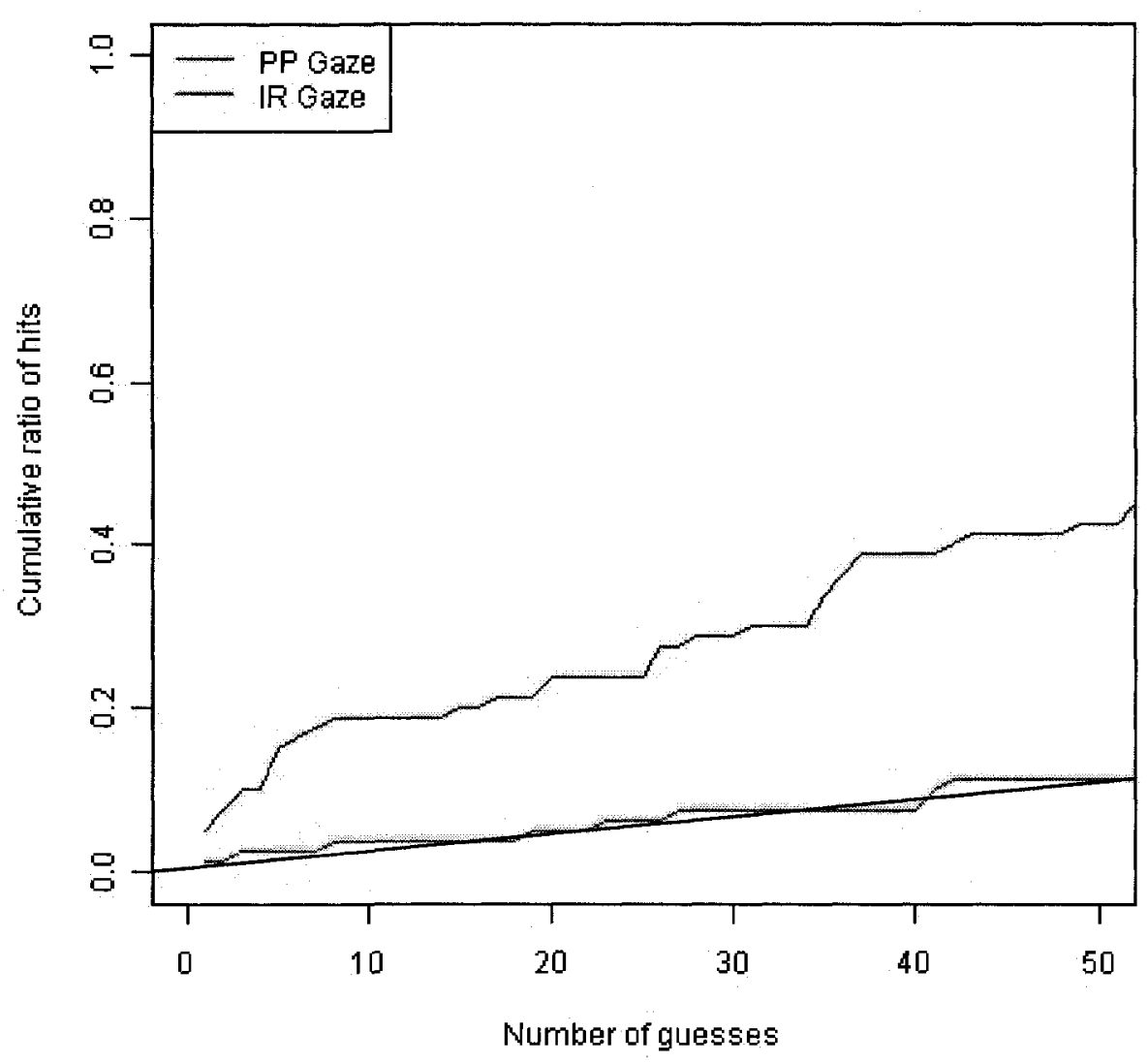

Figure 45. Dictionary attack on the new lab study of the Topdown image, plotted as the cumulative ratio of hits as a function of the number of guesses.

In Figure 45, the results of the dictionary attack demonstrate that the PP gaze distribution correctly guesses a much larger portion of click-points (approximately $43 \%$ after 50 guesses) on the new lab data, when compared to the much lower cumulative ratio of hits for the IR gaze distribution (approximately $9 \%$ after 50 guesses). 


\section{Dictionary attack on the new lab study data (Noise)}

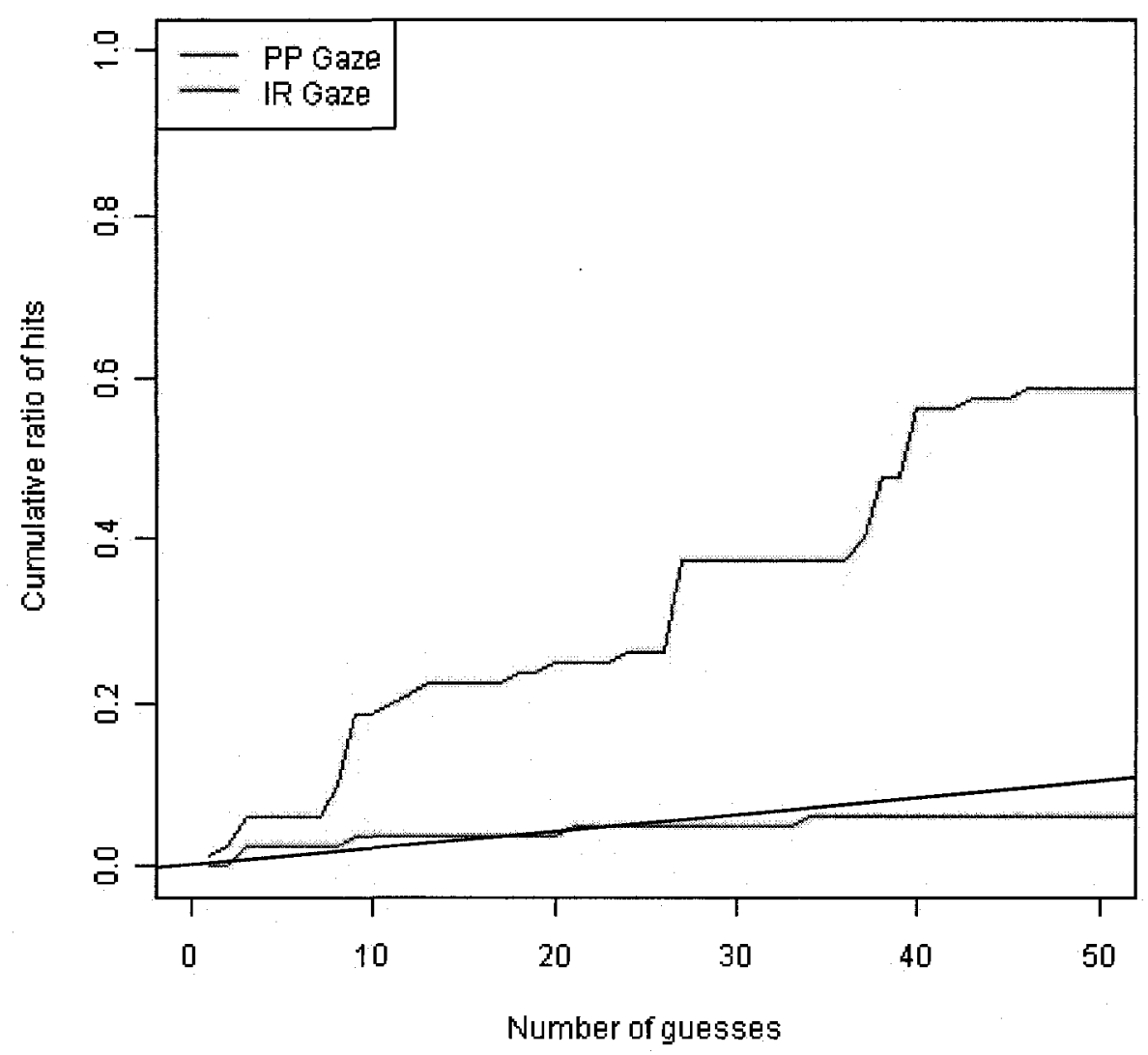

Figure 46. Dictionary attack on the new lab study of the Noise image, plotted as the cumulative ratio of hits as a function of the number of guesses.

The Noise image was one of the more difficult of the images to compare between the PassPoints and the Image Recall conditions, as participants reported very different things during the testing of their respective conditions (e.g. participants in the PP condition looked and clicked in the four corners and the center of the image, while participants in the IR condition stared at different parts of the image). As such, the patterns of the clickpoints were more evenly distributed among the areas of focus of the participants in the PP condition; this is why the above figure demonstrates a much higher cumulative ratio of 
hits for the PP condition (approximately $60 \%$ after 50 guesses) than the IR condition (approximately $2 \%$ after 50 guesses).

\section{Hypothesis 3 Summary: Dictionary Attacks on New Clicks}

We can see from the figures of the dictionary attacks in this section that, in every instance except one (the Statue image), the PassPoints gaze distribution data is more successful at predicting the individual click-points of a particular image than the Image Recall gaze distribution data. Some of the dictionary attacks confirm large differences between the PassPoints and Image Recall conditions (for example, the Noise and Pool images), while others show slight differences between conditions (for example, the Statue and Cars images). Both of the conditions seemed to guess more successfully than would be expected by chance, with the exceptions of the Paperclips and Noise images. Table 18 summarizes the success of the attacks at the level of 20 guesses. By chance, we would expect that 20 guesses would guess approximately 0.05 of the click-points. We choose to highlight 20 guesses as this is an approximation of the point of diminishing returns.

Table 18. Attack dictionary data. The cumulative ratio of hits is depicted for each of the PassPoints and Image Recall conditions at the level of 20 guesses.

\begin{tabular}{lll}
\hline Image & PP & IR \\
\hline Cars & 0.3125 & 0.2250 \\
Pool & 0.5375 & 0.0875 \\
Truck & 0.4000 & 0.1500 \\
Statue & 0.3625 & 0.4000 \\
Paperclips & 0.2500 & 0.0125 \\
Figureground & 0.5000 & 0.3375 \\
Topdown & 0.2375 & 0.0500 \\
Noise & 0.2500 & 0.0375 \\
\hline
\end{tabular}




\section{Dictionary attack on the old lab study data (Cars)}

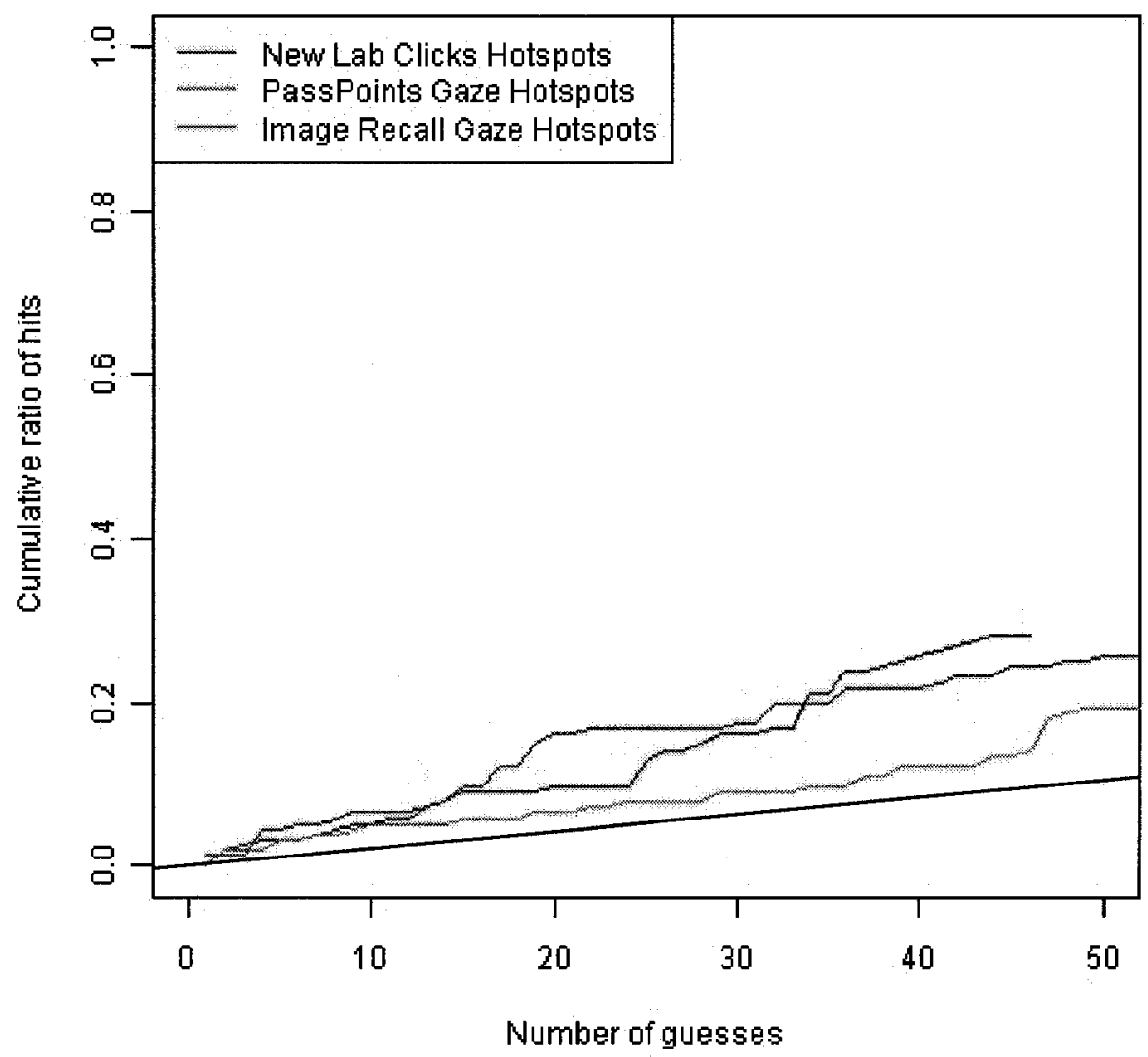

Figure 47. Dictionary attack on the old lab study of the Cars image, plotted as the cumulative ratio of hits as a function of the number of guesses.

In this example, we're attacking the same image, but instead of the field data set, we're attacking the old lab study data. Here, we can see that the new lab click-points data and the Image Recall gaze data are approximately equally strong in finding the old lab study's hotspots, leaving the PassPoints gaze data guessing less points. 
Dictionary attack on the old lab study data (Pool)

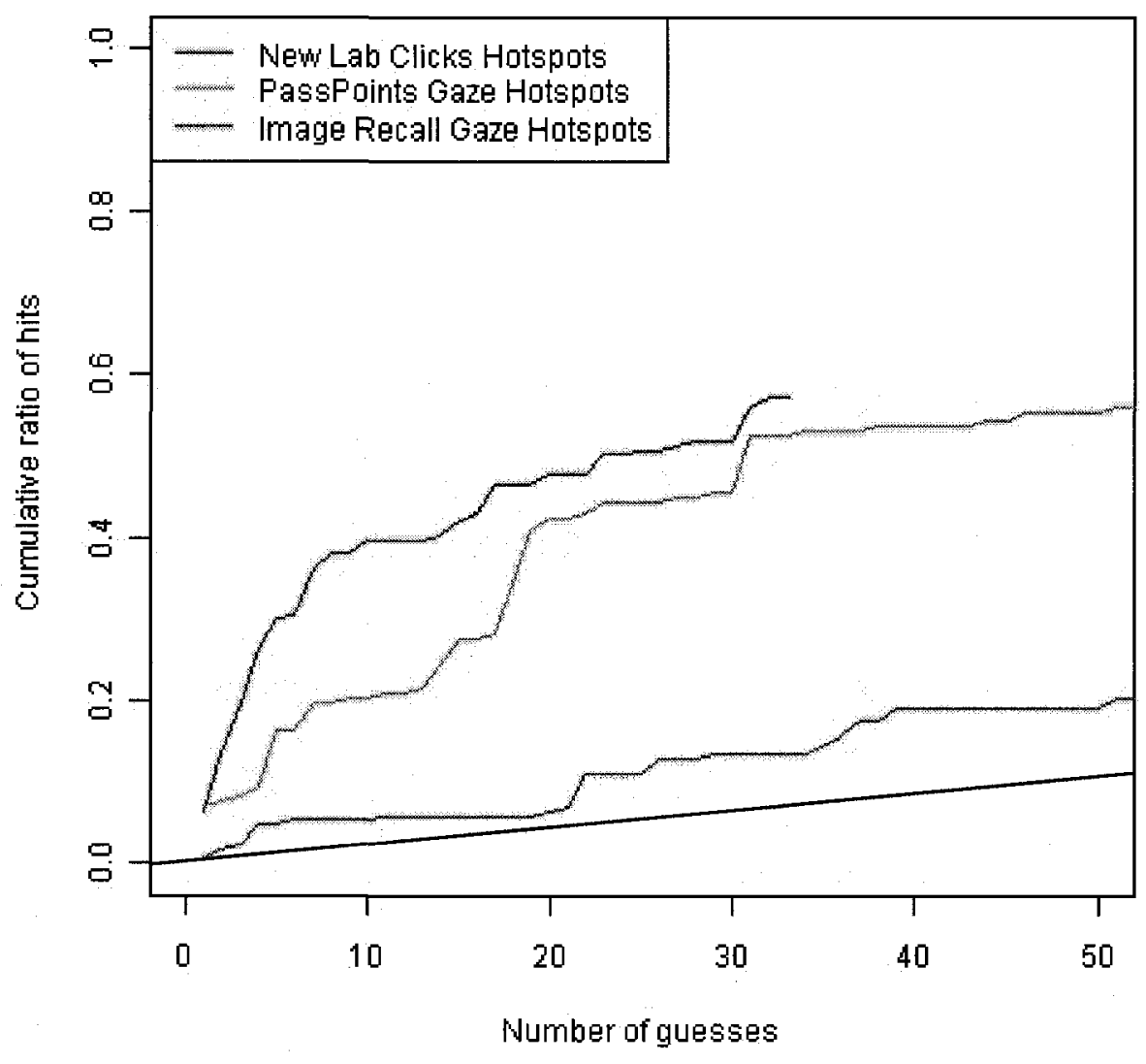

Figure 48. Dictionary attack on the old lab study of the Pool image, plotted as the cumulative ratio of hits as a function of the number of guesses.

In this example, we see that the new lab click-points data guesses more of the hotspots than does the PassPoints gaze data which, in turn, guesses more hotspots than the Image Recall gaze data. 


\section{Dictionary attack on the old lab study data (Truck)}

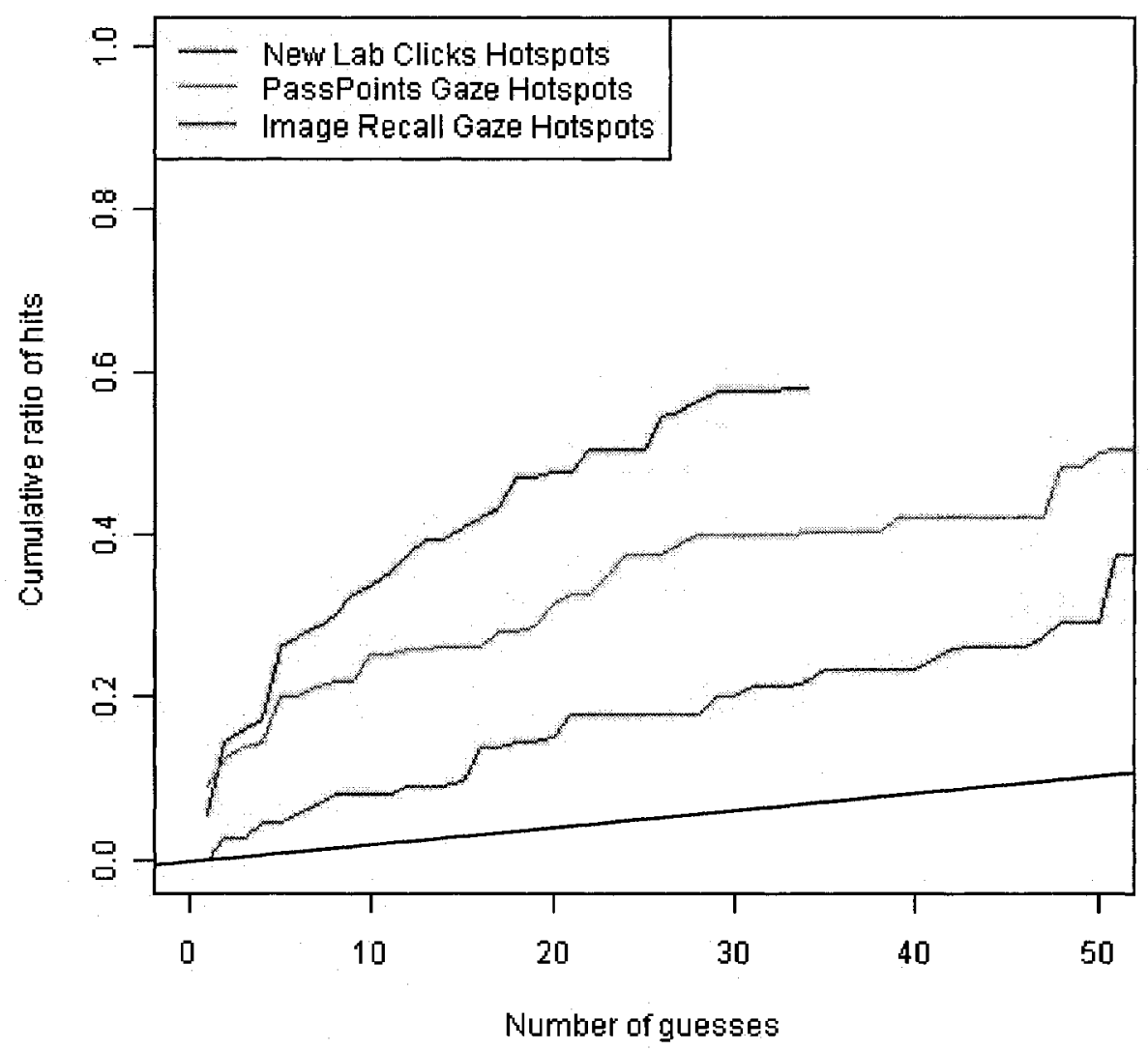

Figure 49. Dictionary attack on the old lab study of the Truck image, plotted as the cumulative ratio of hits as a function of the number of guesses.

For the Truck image, our dictionary attack on the old lab click-points data shows that the new lab click-points data is more efficient in finding hotspots than the PassPoints and Image Recall gaze data, but that these are evenly spaced between one another, unlike the cars image, which saw the three separate groups appear much more closely in terms of their efficiency of attack. 


\section{Dictionary attack on the old lab study data (Paperclips)}

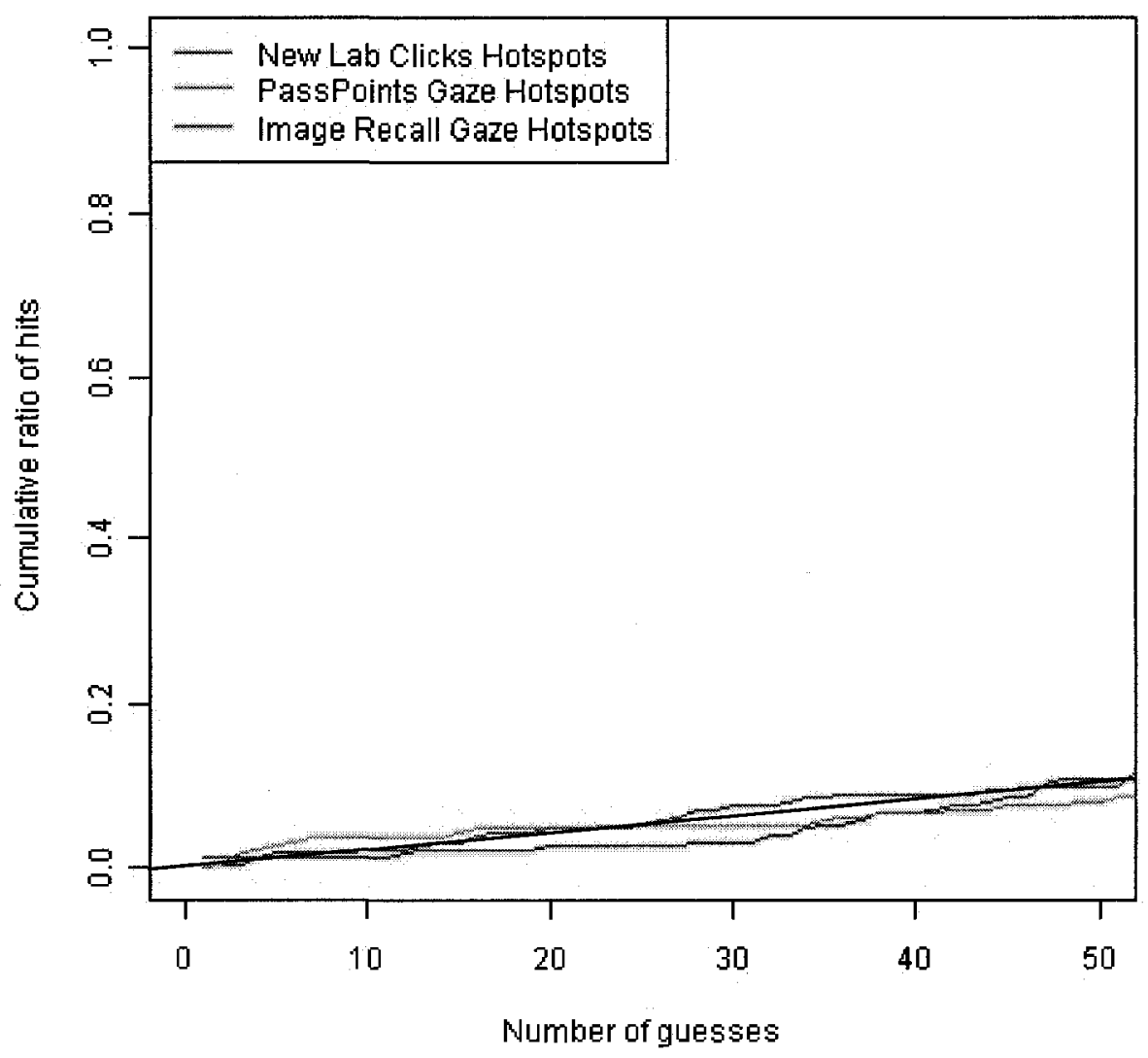

Figure 50. Dictionary attack on the old lab study of the Paperclips image, plotted as the cumulative ratio of hits as a function of the number of guesses.

In this example, we see that each of the three groups, the new lab click-points data, the PassPoints gaze data, as well as the Image Recall gaze data, are equally non-efficient in finding the hotspots of the old lab study click-points. This is apparent by the lack of a high cumulative ratio of hits as a function of the number of guesses in the attack dictionary. We suggest that this is because users had no reason to look or gaze at any one of the paperclips anymore than any other paperclips. Note that while this is a good result from a security point of view, Chiasson et al. (2007) reported that this image was the one where users had the greatest difficulty in remembering click-points. 


\section{Dictionary attack on the old lab study data (Statue)}

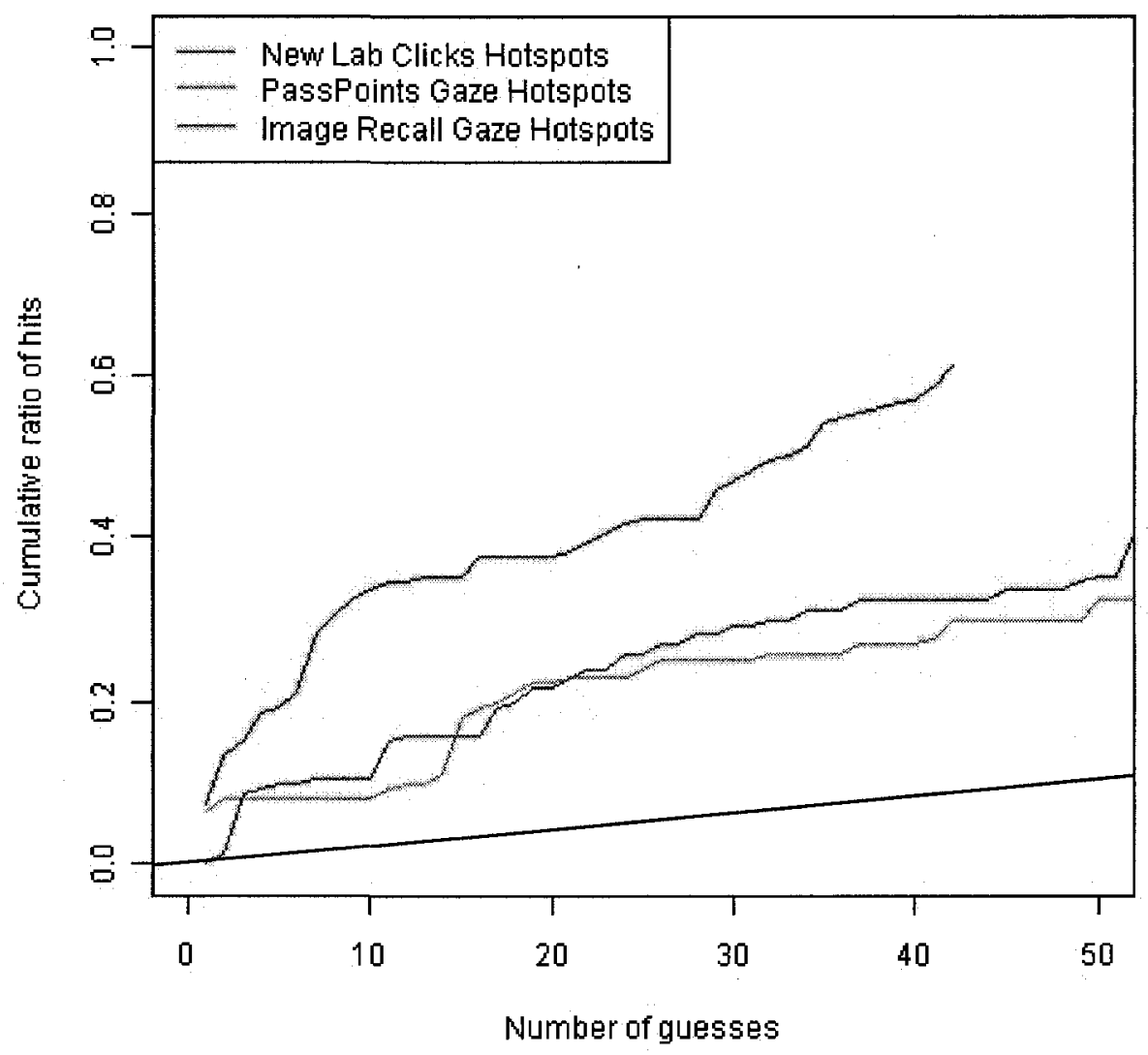

Figure 51. Dictionary attack on the old lab study of the Statue image, plotted as the cumulative ratio of hits as a function of the number of guesses.

In the final graph of the attack dictionary, we see that the new lab click-points data manages to guess approximately double the amount of the click-points, between 20 and 30 guesses, than do the other two groups, the PassPoints and Image Recall gaze distributions. 


\section{Dictionary attack on the field study data (Cars)}

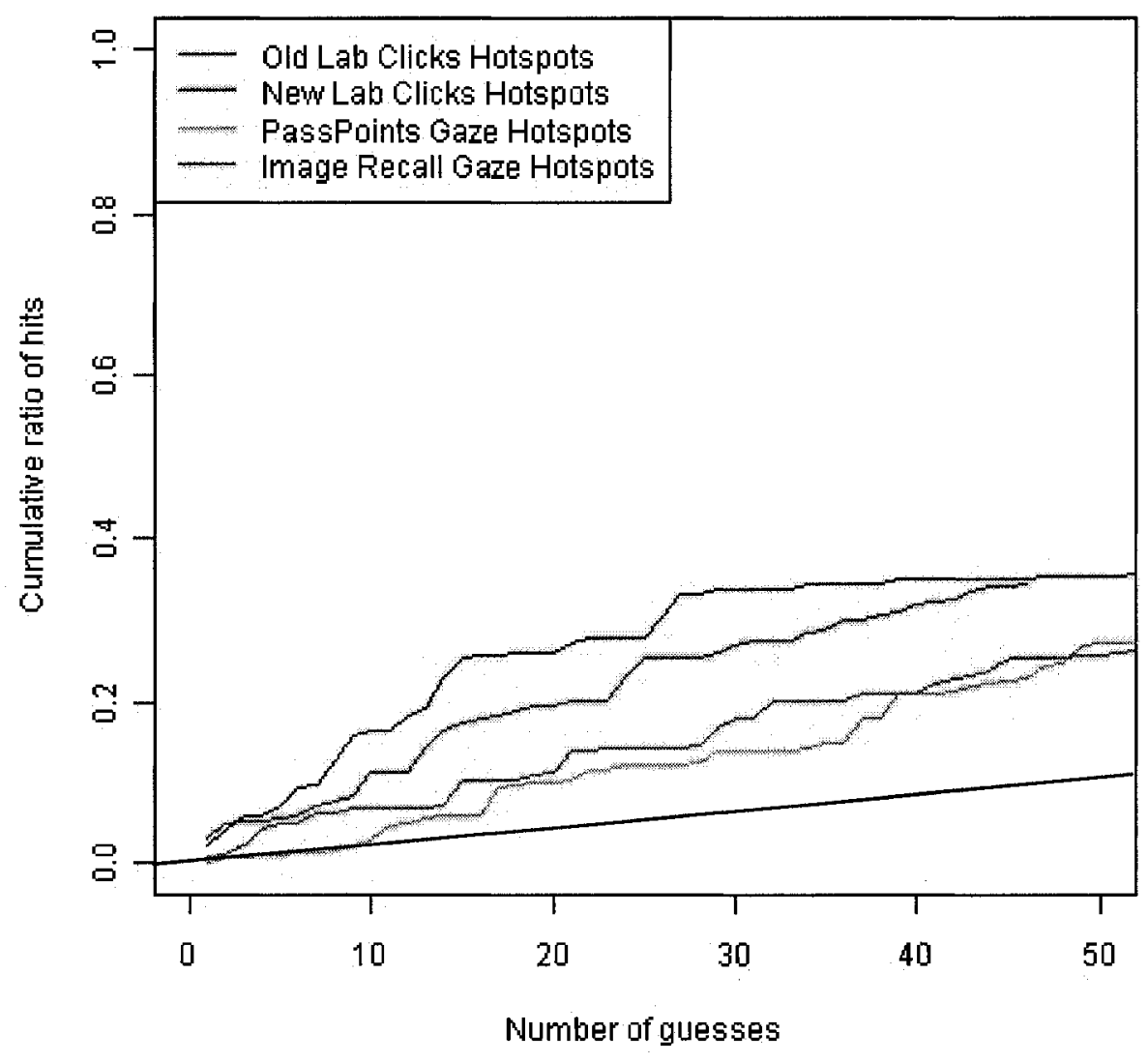

Figure 52. Dictionary attack on the field study of the Cars image, plotted as the cumulative ratio of hits as a function of the number of guesses.

In this particular set of dictionary attacks, we're targeting the field data of the Cars image. As we can see by Figure 52, the old lab click-points data guesses a few more hotspots than the new lab click-points data; however, both of these guess a few more hotspots than the gaze hotspots for both the PassPoints and the Image Recall conditions. 


\section{Dictionary attack on the field study data (Pool)}

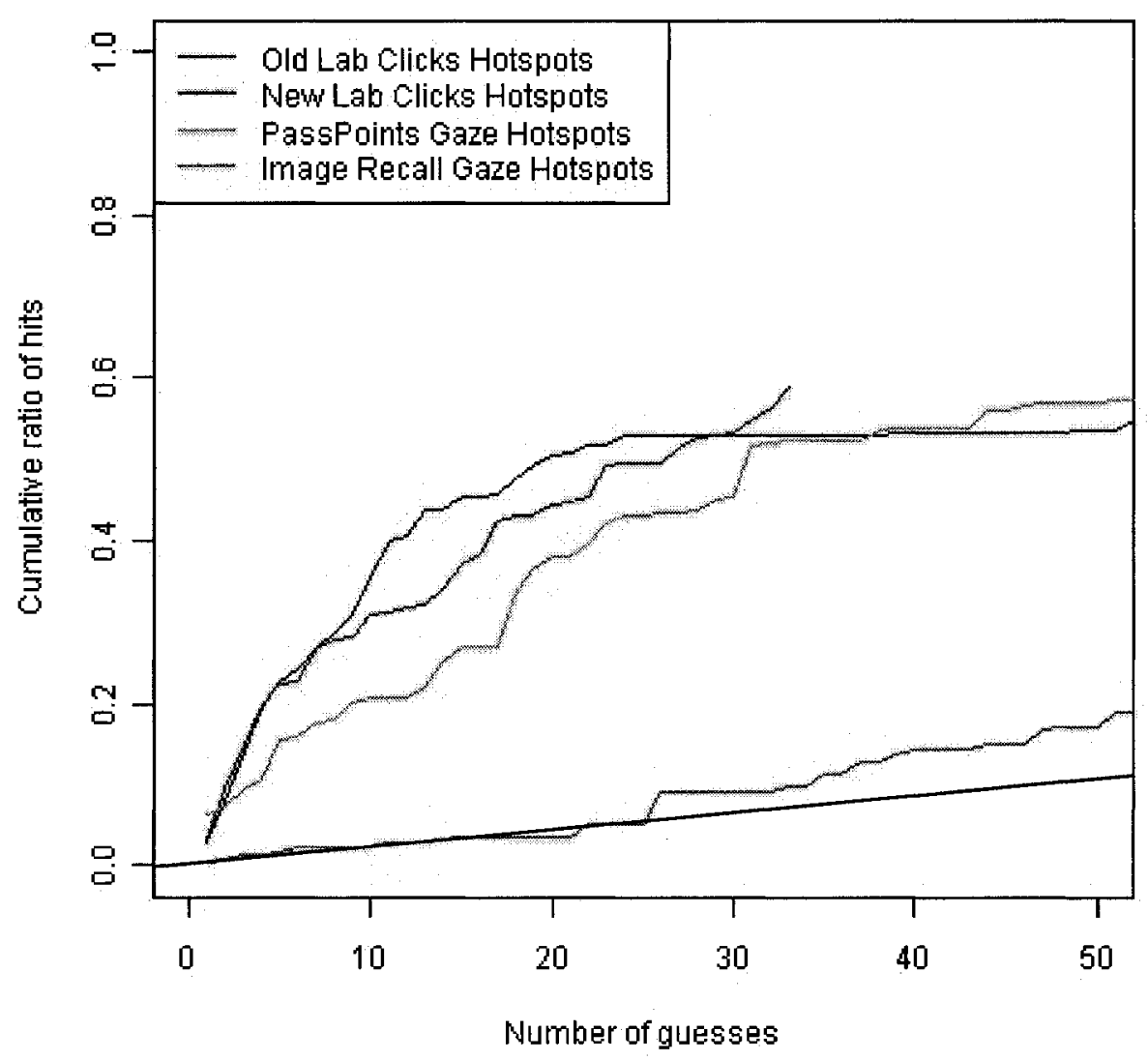

Figure 53. Dictionary attack on the field study of the Pool image, plotted as the cumulative ratio of hits as a function of the number of guesses.

This graph demonstrates the attack on the field data of the Pool image. We can see that the old lab click-points data, the new lab click-points data, and the PassPoints gaze data closely follow each other in the number of hits they encounter along their road to the final guess, leaving the Image Recall gaze data at a distant fourth. The line for the Image Recall attack is very similar to what we would expect by chance. 
Hypothesis 3 Summary: Dictionary Attacks on Old Clicks and Field Clicks

When using the PassPoints and Image Recall gaze data as well as the new lab study click-point data to attack the old lab study click-point data, we found that all cases were more successful at guessing than we would expect by chance with the exception again of the Paperclips and Noise images. In attacking the field data, the Image Recall gaze data was also to results that might occur by chance. The new lab study click-point data is in most cases the most successful at predicting individual click-points. Furthermore, we can see from the figures of the dictionary attacks on the field study that, in most instances, both the new study click-point data and the old study click-point data are more successful at predicting the individual click-points of a particular image than either the PassPoints or Image Recall gaze distribution data. In most cases, there are minimal differences between the new lab study and old lab study click-point data, as well as between the PassPoints and Image Recall gaze distribution data in predicting individual click-points, but there are larger differences between both sets of data distributions. This is not always the case, as we can see from the Paperclips image, where all of the data distributions closely follow one another in terms of the cumulative ratio of hits plotted against the number of guesses. Table 19 depicts the comparisons between the new clicks and the old clicks, as well as the gaze distribution data for both the PassPoints and Image Recall conditions. The patterns of the gaze distribution data for both the PassPoints and Image Recall conditions, as well as the patterns for the new lab study overlap the old lab study click-point patterns. These three patterns, as well as the old lab study click-point patterns, overlap the field study click-points, thus showing that hypothesis 3 can potentially be justified from this analysis of the dictionary attacks. 
Table 19. Comparison of analytical results for the Dictionary Attack Analysis section of Hypothesis 3. The * indicates that there is no available data for the particular item in the table.

\begin{tabular}{llll}
\hline $\begin{array}{l}\text { Comparison of Analytical Results } \\
\text { Dictionary Analysis }\end{array}$ & New Clicks & Old Clicks & \\
Target: & Of Gaze Patterns & Of Gaze Patterns & Overall \\
Best Attack: & IR & PP & Clicks \\
\hline Cars & Similar & Similar & Clicks \\
Pool & PP & PP & Clicks \\
Statue & IR & PP & Clicks \\
Truck & PP & Similar & Similar \\
PaperClips & PP & $*$ & $*$ \\
TopDown & IR & $*$ & $*$ \\
FigureGround & PP & $*$ & $*$ \\
Noise & & & \\
\hline
\end{tabular}

In the preceding analyses, the more complex results are those that relate to the earlier study by Chiasson et al. (2007). We ran a repeated measures Analysis of Variance on the resulting data from the attack dictionaries, in order to determine which of three groups was significantly different from the others in terms of the number of guesses it took to obtain a significant number of correct guesses of the image hotspots. Our analysis comprised two levels: the first level was the type of attack (new clicks hotspots, PassPoints gaze hotspots, and Image Recall gaze hotspots); the second category was the number of hits level based on the number of guesses ( 5 guesses, 10 guesses, 15 guesses, and 20 guesses). The data found within each of the level of hits depicts the percentage of click-points guessed at each level. For example, for the Cars image, within the New Clicks attack type and at a level of 10 hits, the percentage of click-points guessed was roughly $5 \%$. The raw data for the analysis of variance is shown in Table 20 below. 
Table 20. Attack dictionary data used in the analysis of variance. The data in each cell for the level of hits category reflects the proportion of correct guesses for each of four levels, $5,10,15$, and 20 guesses. .

\begin{tabular}{l|lcccc}
\hline \multirow{2}{*}{ Attack Type } & Image & $\mathbf{5}$ & $\mathbf{1 0}$ & $\mathbf{1 5}$ & $\mathbf{2 0}$ \\
\hline New Clicks & Cars & 0.032258 & 0.051613 & 0.090323 & 0.096774 \\
& Pool & 0.300578 & 0.398844 & 0.421965 & 0.479769 \\
& Truck & 0.262857 & 0.337143 & 0.411429 & 0.480000 \\
& Paperclips & 0.013636 & 0.013636 & 0.022727 & 0.027273 \\
PP Gaze & Statue & 0.194118 & 0.335294 & 0.352941 & 0.376471 \\
& Cars & 0.032258 & 0.051613 & 0.058065 & 0.064516 \\
& Pool & 0.161850 & 0.202312 & 0.277457 & 0.427746 \\
& Truck & 0.200000 & 0.251429 & 0.262857 & 0.314286 \\
& Paperclips & 0.027273 & 0.036364 & 0.045455 & 0.050000 \\
& Statue & 0.082353 & 0.082353 & 0.182353 & 0.223529 \\
& Cars & 0.045161 & 0.064516 & 0.096774 & 0.161290 \\
& Pool & 0.046243 & 0.052023 & 0.057803 & 0.063584 \\
& Truck & 0.045714 & 0.080000 & 0.097143 & 0.148571 \\
& Paperclips & 0.018182 & 0.022727 & 0.031818 & 0.050000 \\
& Statue & 0.100000 & 0.105882 & 0.158824 & 0.217647 \\
\hline
\end{tabular}

The resulting two-way within-subjects analysis of variance found that although there was no significant effect for the attack type, $F(2,30)=3.5906, p=0.074$, there was a significant effect of the levels variable, which we would expect, $F(1,30)=17.836, p=$ 0.013 , and there was no interaction between independent variables, $F(2,30)=0.8909, p=$ 0.4474 .

JCross Analysis for Hypothesis 3

In our data analysis, we were interested in determining whether point patterns from the gaze distributions for either of the two conditions (PassPoints and Image Recall) would overlap patterns in click-points data. One way to study this is to plot two data sets overlapping each other using the JCross statistic (Van Lieshout \& Baddeley, 1999). This statistic takes one data set, and compares each point with the points in the other data set, calculating a measure of how similar the two data sets are in terms of distance and 
clustering. This statistic is calculated relative to a radius $r$, indicating the proximity of interest of comparison. To show a comparison of two point patterms, we create a plot with increasing radii on the horizontal axis, and the JCross statistic on the vertical axis. Where the resulting plot line stays close to 1 , it provides evidence for the randomness of the data sets in comparison to one another; this basically means that they are independent of one another. However, if the plot line tends towards 0 , then there is evidence of a pattern match between the two data sets. If the plot line tends above 1 , it indicates that the patterns "repulse" each other, suggesting a kind of inverse relationship (see Appendix N).

The figures below demonstrate our results. In each of these graphs, the $\mathrm{X}$ axis depicts the radius of each cross comparison. The $\mathrm{Y}$ axis represents the JCross statistic. Each of the JCross graphs found below include a Monte Carlo randomization calculation, where 19 random distributions are created and compared to the resulting JCross calculation for each data set (PPGazeCars versus OldCarsClicks, as an example). The graphs show the upper bound and lower bound of the Monte Carlo randomization calculations. For most of our analyses, we're interested in the lower bound. If the JCross calculation for the data set in question is found to be below the lower bound of the Monte Carlo randomization calculation, then this indicates that the similarity between the two data sets is significant at that level; for example, 20 data sets indicates an alpha level of $5 \%$.

The tolerance of PassPoints involves squares of 19 by 19 pixels. Since PassPoints has a square tolerance region, and JCross is based on circles described by a radius, an approximate radius of 9 to 11 would work best to ensure that we included the same area 
within the tolerance region of PassPoints. We therefore pay most attention to the graph up to a radius of approximately 9 to 11 pixels.

Figures 54 through 61 show the JCross statistic comparing both the PassPoints gaze data and the Image Recall gaze data with the new lab study click-point data for each image in turn. We then summarize our findings, and then consider the overall findings for hypothesis 3 . Figures 63 through 67 compared both gaze conditions and the new lab study click-point data to the old lab study click-point data. We then summarize these findings.
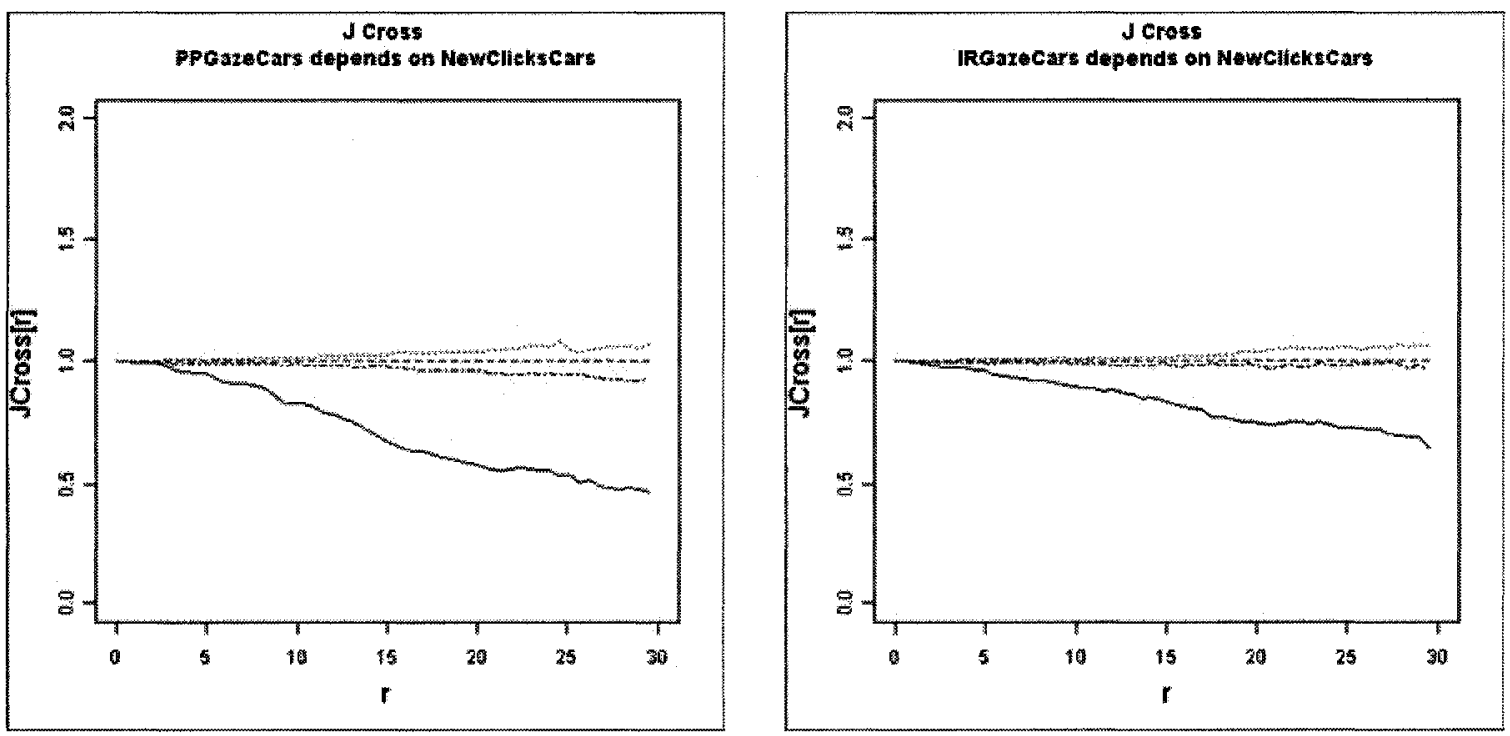

Figure 54. JCross graphs on the Cars image: the PassPoints gaze distribution data (top-left) and the ImageRecall gaze distribution data (top-right) plotted against the new lab click-points data. 

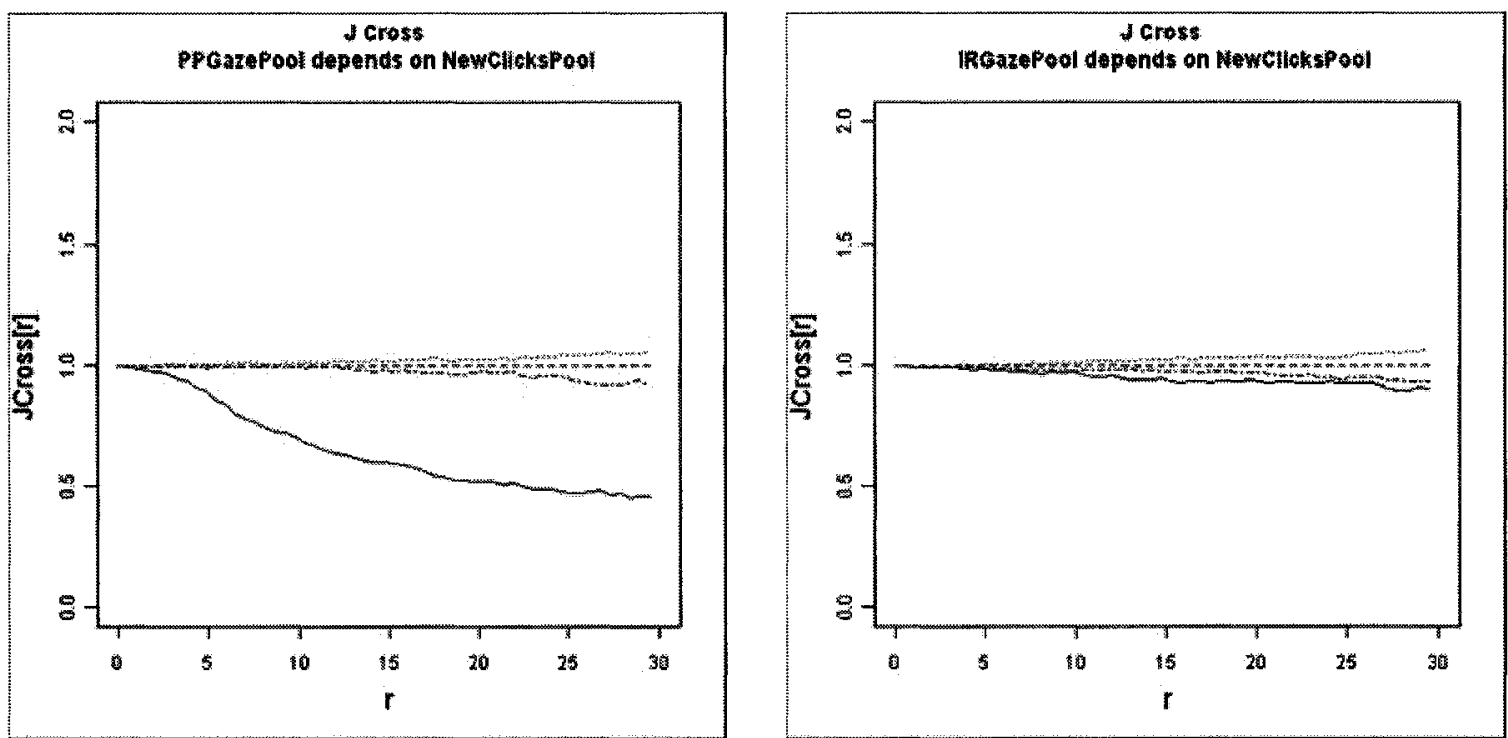

Figure 55. JCross graphs on the Pool image: the PassPoints gaze distribution data (top-left) and the ImageRecall gaze distribution data (top-right) plotted against the new lab click-points data.
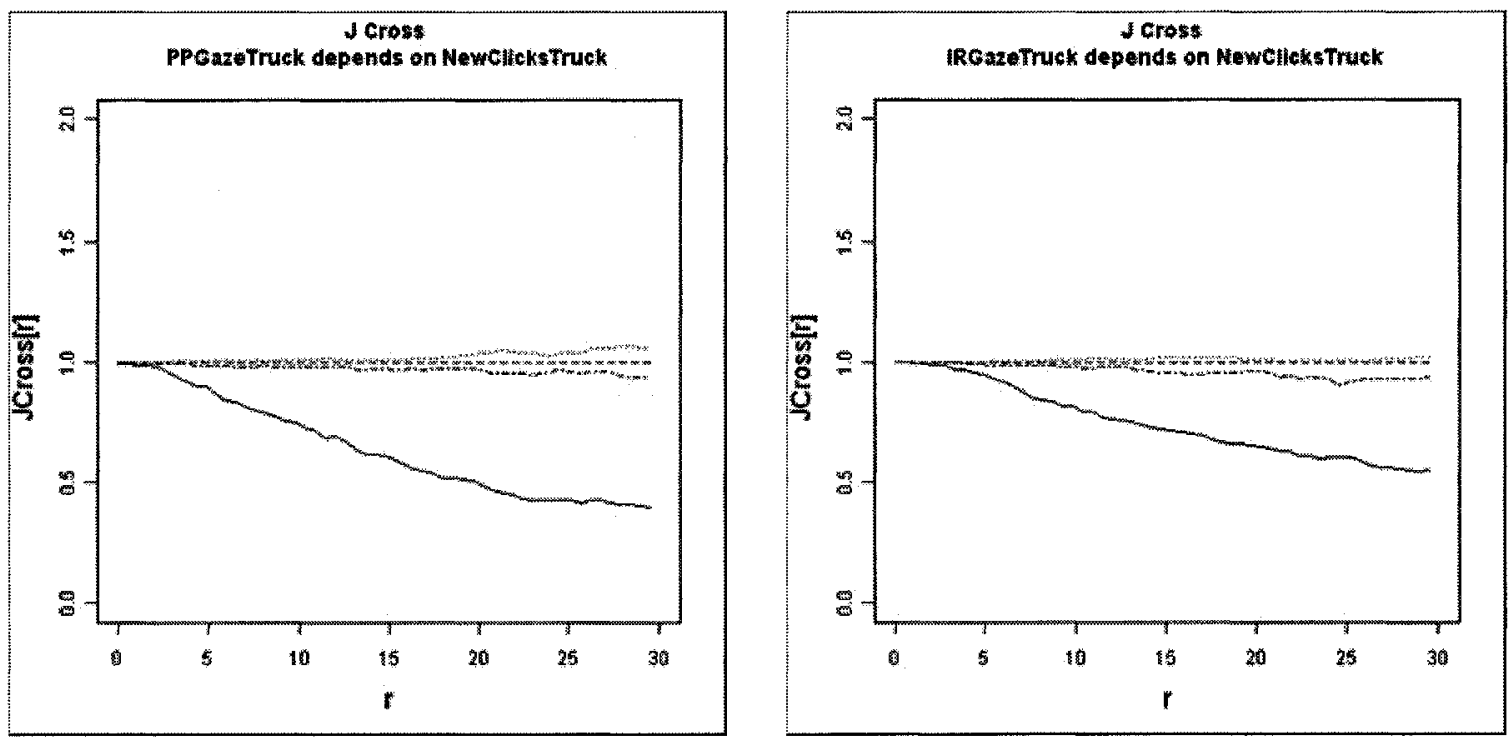

Figure 56. JCross graphs for the Truck image: the PassPoints gaze distribution data (top-left) and the ImageRecall gaze distribution data (top-right) plotted against the new lab click-points data. 

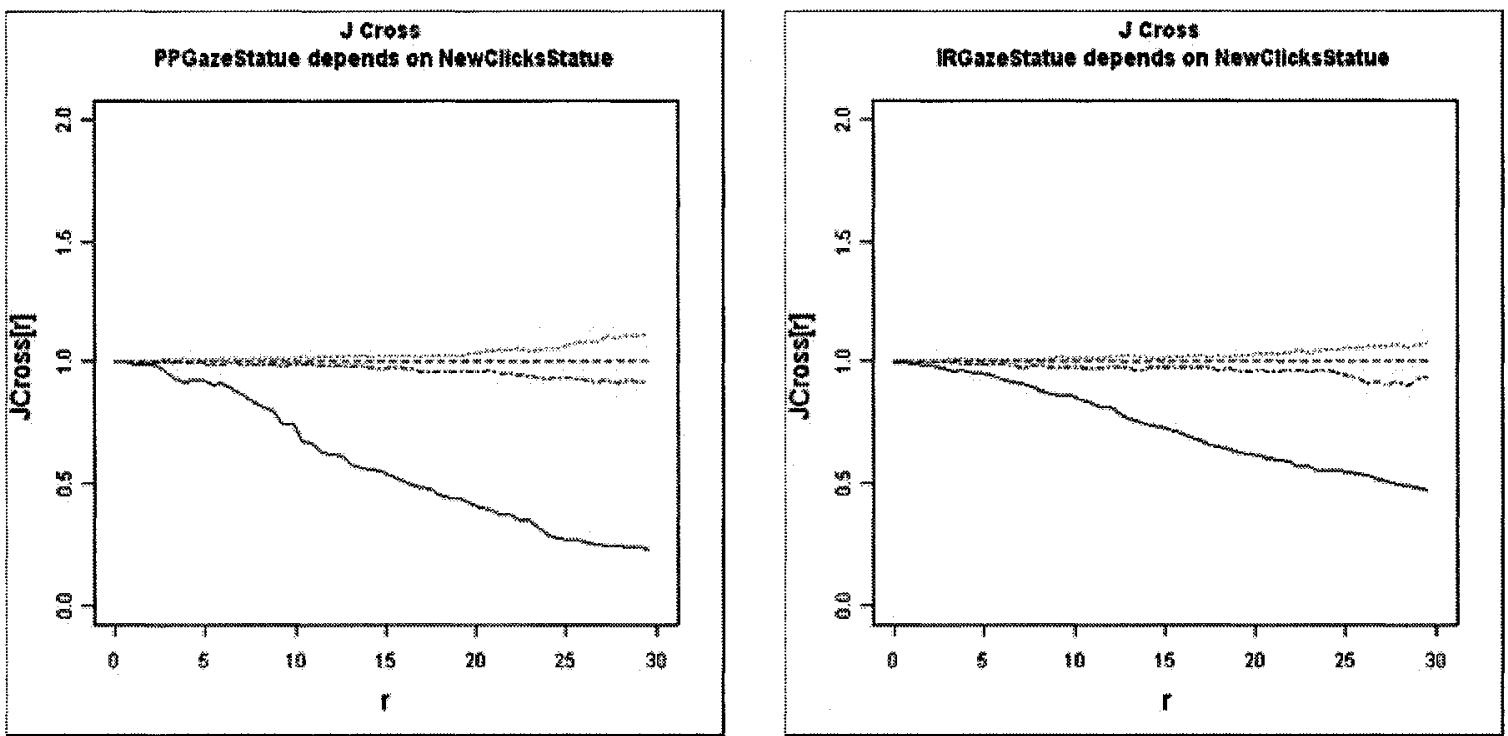

Figure 57. JCross graphs for the Statue image: the PassPoints gaze distribution data (top-left) and the ImageRecall gaze distribution data (top-right) plotted against the new lab click-points data.
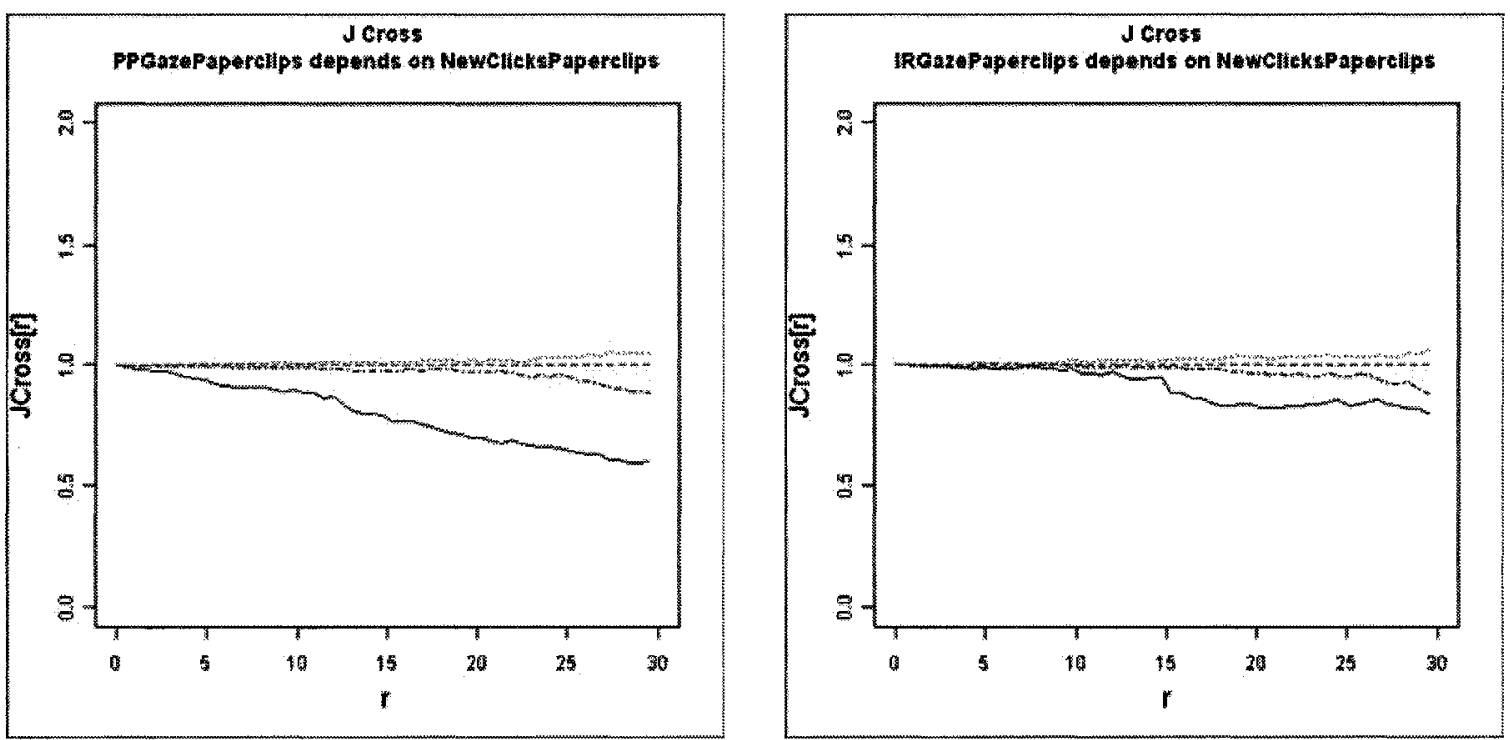

Figure 58. JCross graphs for the Paperclips image: the PassPoints gaze distribution data (top-left) and the ImageRecall gaze distribution data (top-right) plotted against the new lab click-points data. 

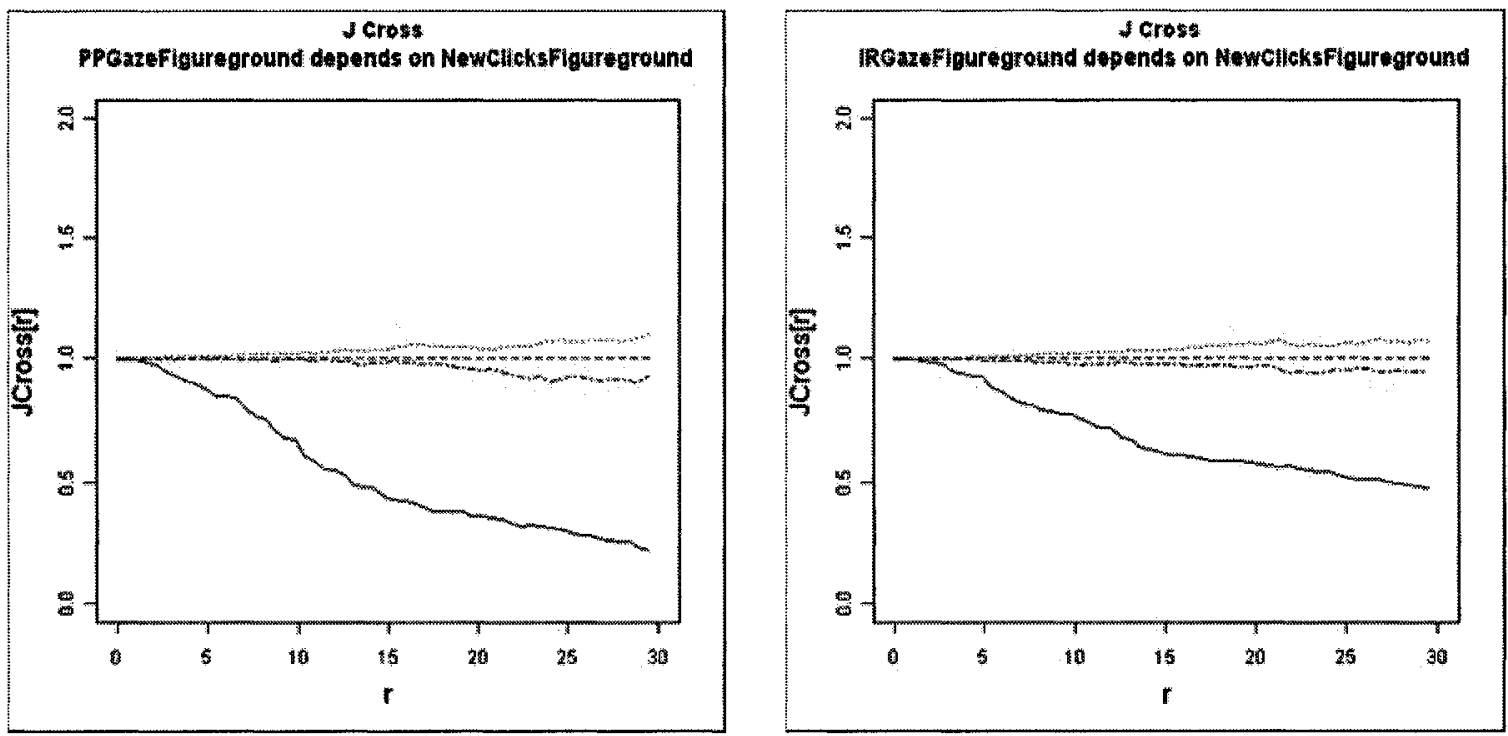

Figure 59. JCross graphs for the Figureground image: the PassPoints gaze distribution data (top-left) and the ImageRecall gaze distribution data (top-right) plotted against the new lab click-points data.
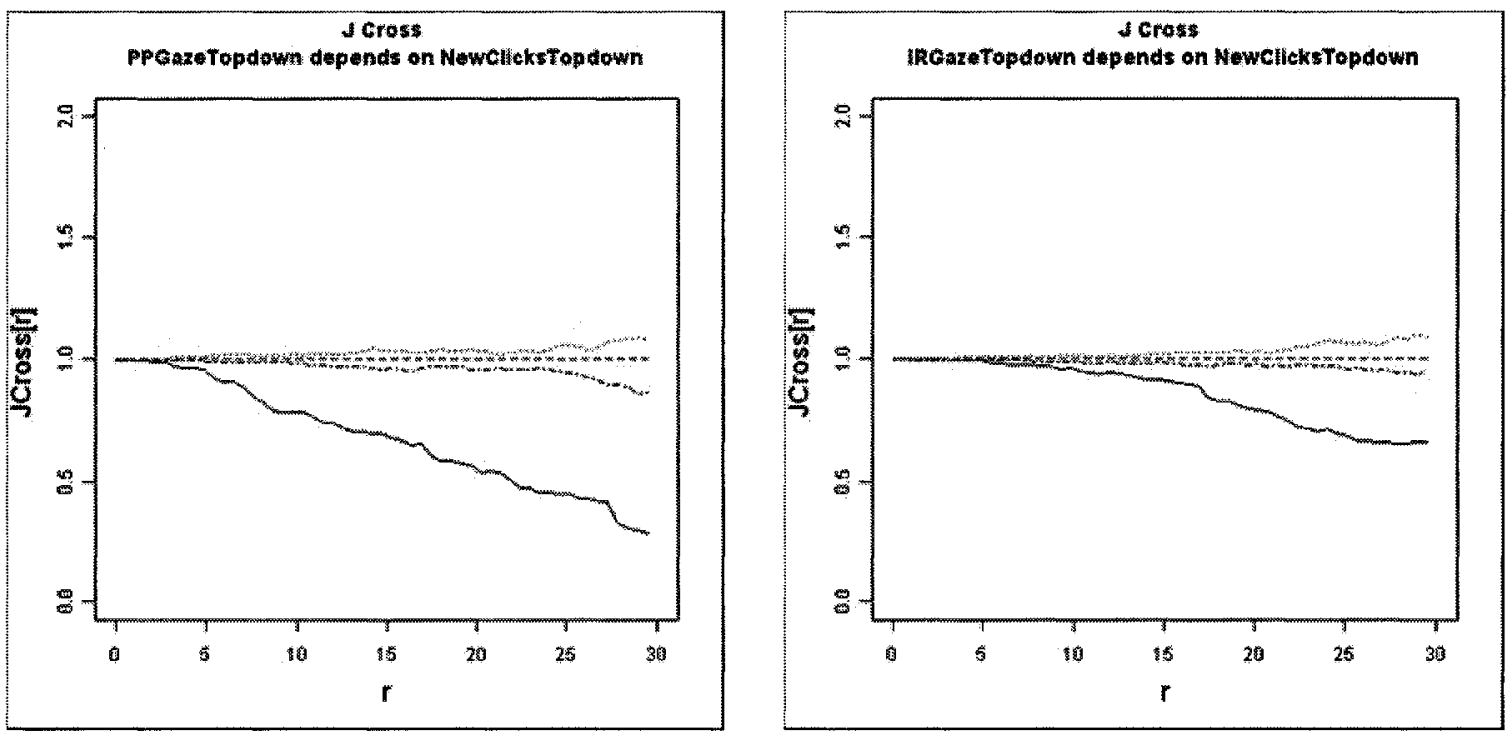

Figure 60. JCross graphs for the Topdown image: the PassPoints gaze distribution data (top-left) and the ImageRecall gaze distribution data (top-right) plotted against the new lab click-points data. 

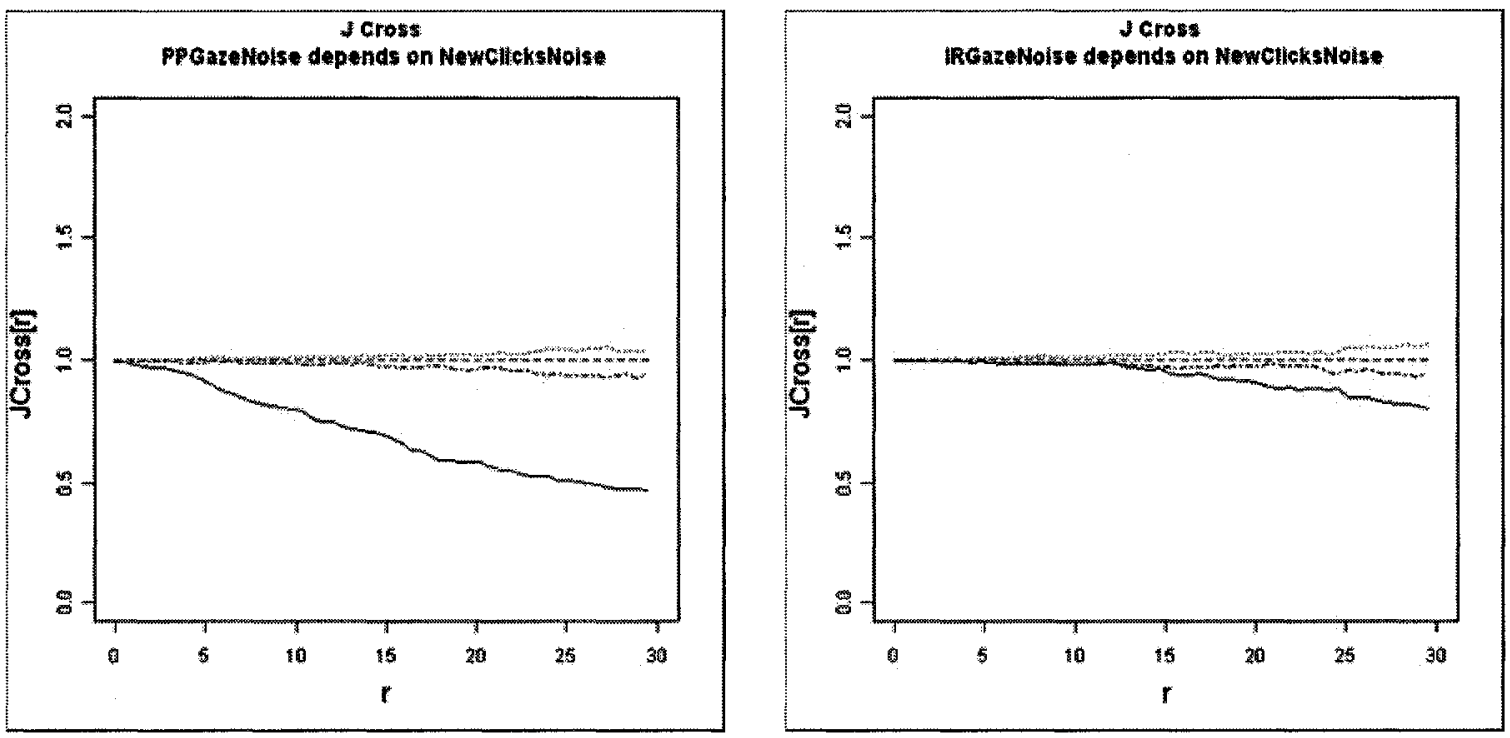

Figure 61. JCross graphs for the Noise image: the PassPoints gaze distribution data (top-left) and the ImageRecall gaze distribution data (top-right) plotted against the new lab click-points data.

\section{Hypothesis 3 Summary: JCross Patterns for New Clicks}

We tested each of the gaze data distributions (PassPoints and Image Recall), as well as against the new lab study click-point data. The JCross comparisons between each of these, demonstrate that there are similarities between them for the most part. In almost every instance (except for the Paperclips and Noise images), the patterns of the gaze distribution plotted against the new lab study click-point data demonstrates significance. We can see this by looking at the Monte Carlo lines drawn on each of the JCross graphs. If the trend line between the $9^{\text {th }}$ and $11^{\text {th }}$ radius point is found to be below the randomly generated dotted lines, then the patterns are said to be significantly similar to one another. Except for the two images mentioned, each of these comparisons yielded significant results, which provides evidence to support our third hypothesis. 
Table 21. The randomness of the JCross patterns for both PassPoints and Image Recall gaze data, plotted against the new lab study click-point data at a radius of $r=10$.

\begin{tabular}{l|cc}
\hline \multicolumn{1}{c|}{ Image } & PassPoints & Image Recall \\
\hline Cars & 0.827 & 0.893 \\
Pool & 0.693 & 0.970 \\
Truck & 0.741 & 0.808 \\
Statue & 0.701 & 0.849 \\
Paperclips & 0.889 & 0.973 \\
Figureground & 0.638 & 0.763 \\
Topdown & 0.784 & 0.960 \\
Noise & 0.801 & 0.985 \\
\hline
\end{tabular}

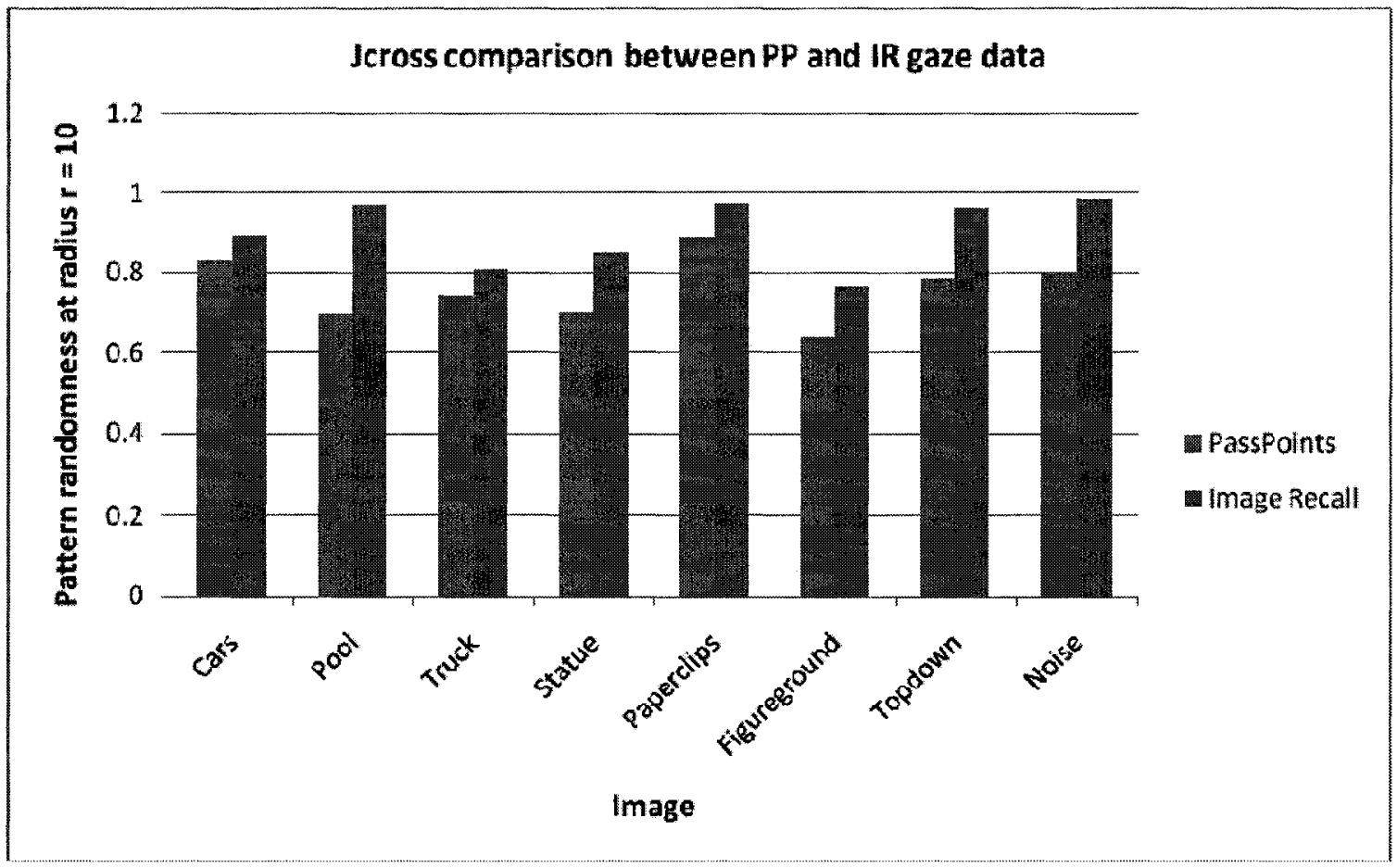

Figure 62. The randomness of the JCross patterns for both PassPoints and Image Recall gaze data, plotted against the new lab study click-point data at a radius of $r=10$.

It is apparent from Table 21 and Figure 62 that, between the gaze data and the new lab study click-point data, the randomness of the patterns is more present in the Image Recall condition. For each of the eight images, the gaze data distribution from the Image Recall condition is, each time, closer to 1.0 on the graph at a radius of 10 pixels. We know that 
as the line drops towards 0.0 the randomness of the patterns becomes more and more unlikely, this provides evidence that the PassPoints gaze data is always more similar to the new lab study click-point pattern.

We now turn to the results relating to the older study data in Figures 63 through

67. 

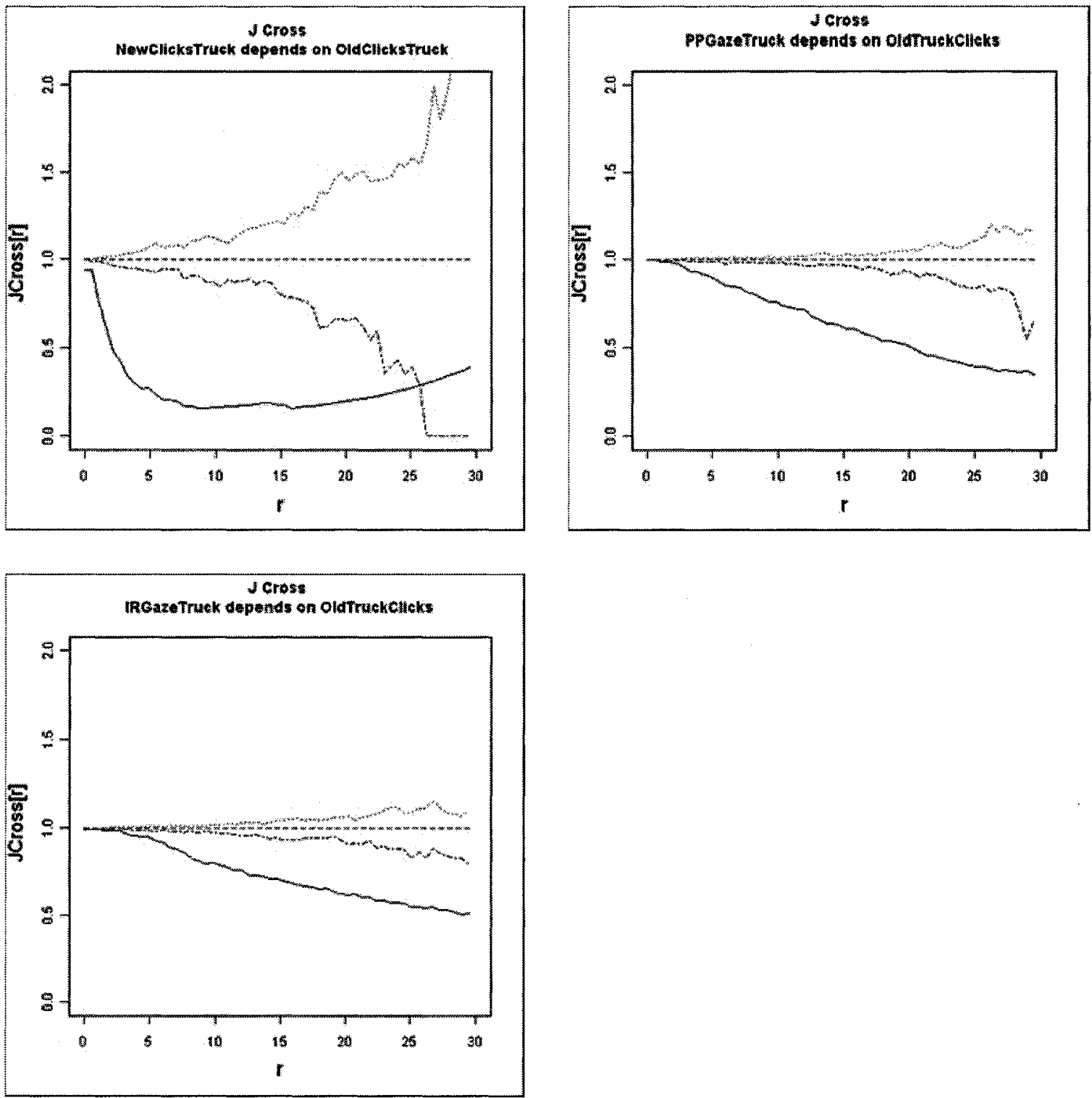

Figure 63. JCross graphs based on similarities between the Truck image new lab click-points data (top-left), PassPoints gaze data (top-right), and Image Recall gaze data (bottom-left), when compared to the Statue old lab study click-points data. 

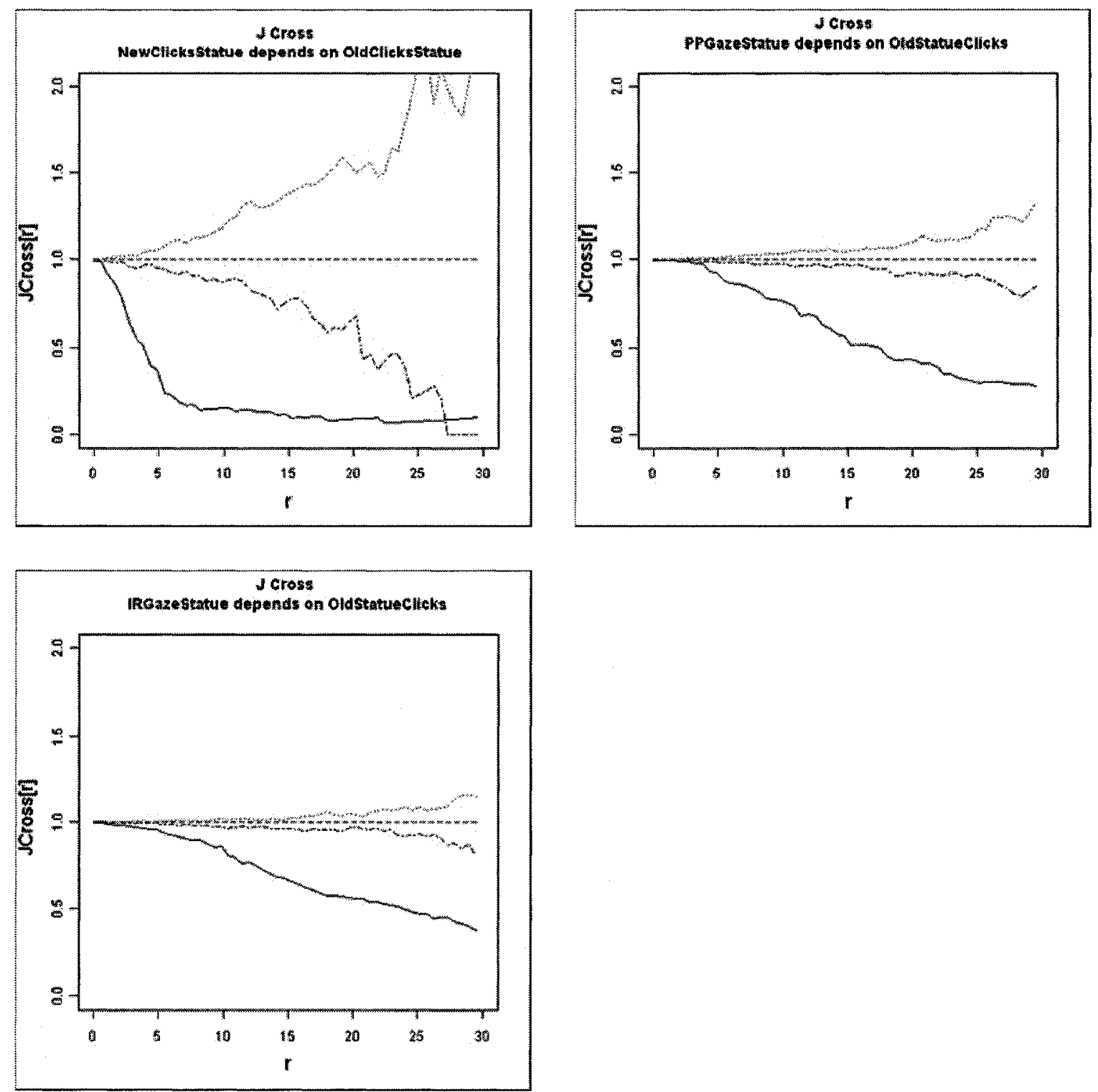

Figure 64. JCross graphs based on similarities between the Statue image new lab click-points data (top-left), PassPoints gaze data (top-right), and Image Recall gaze data (bottom-left), when compared to the Statue old lab study click-points data. 

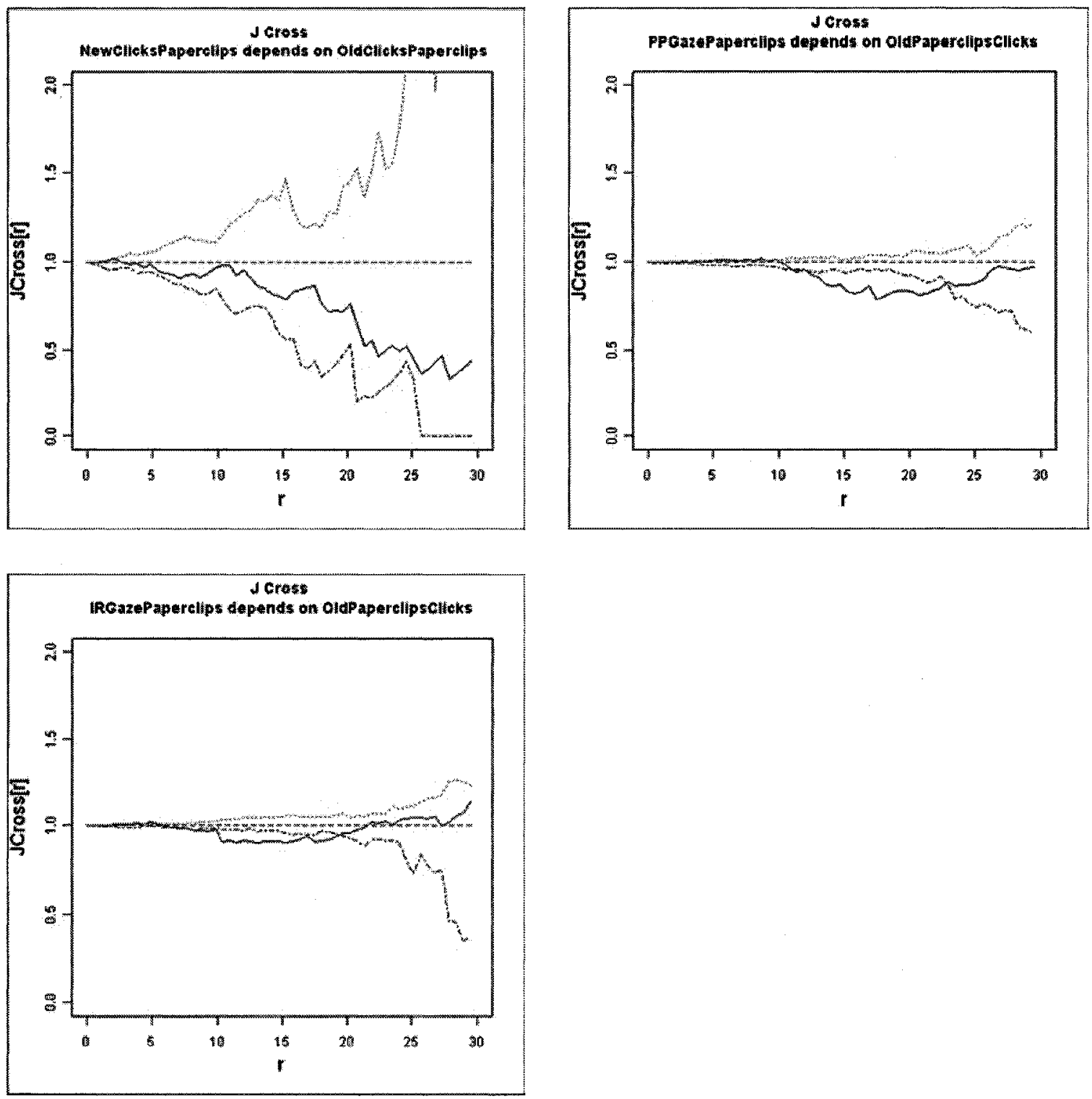

Figure 65. JCross graphs based on similarities between the Paperclips image new lab click-points data (top-left), PassPoints gaze data (top-right), and Image Recall gaze data (bottom-left), when compared to the Paperclips old lab study click-points data. 

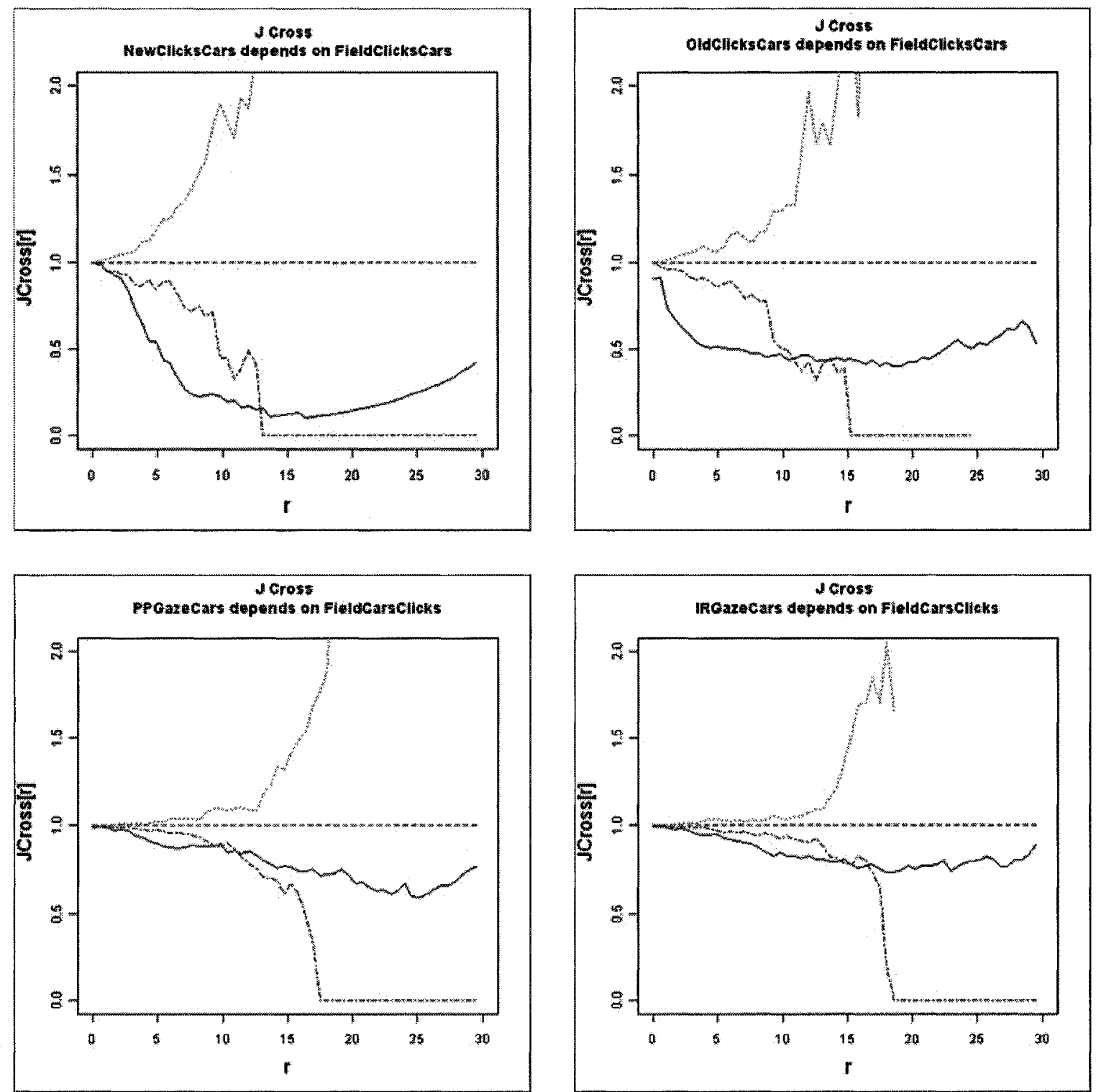

Figure 66. JCross graphs based on similarities between the Cars image old lab click-points data (topleft), new lab click-points data (top-right), PassPoints gaze data (bottom-left), and Image Recall gaze data (bottom-right), when compared to the Cars field click-points data. 

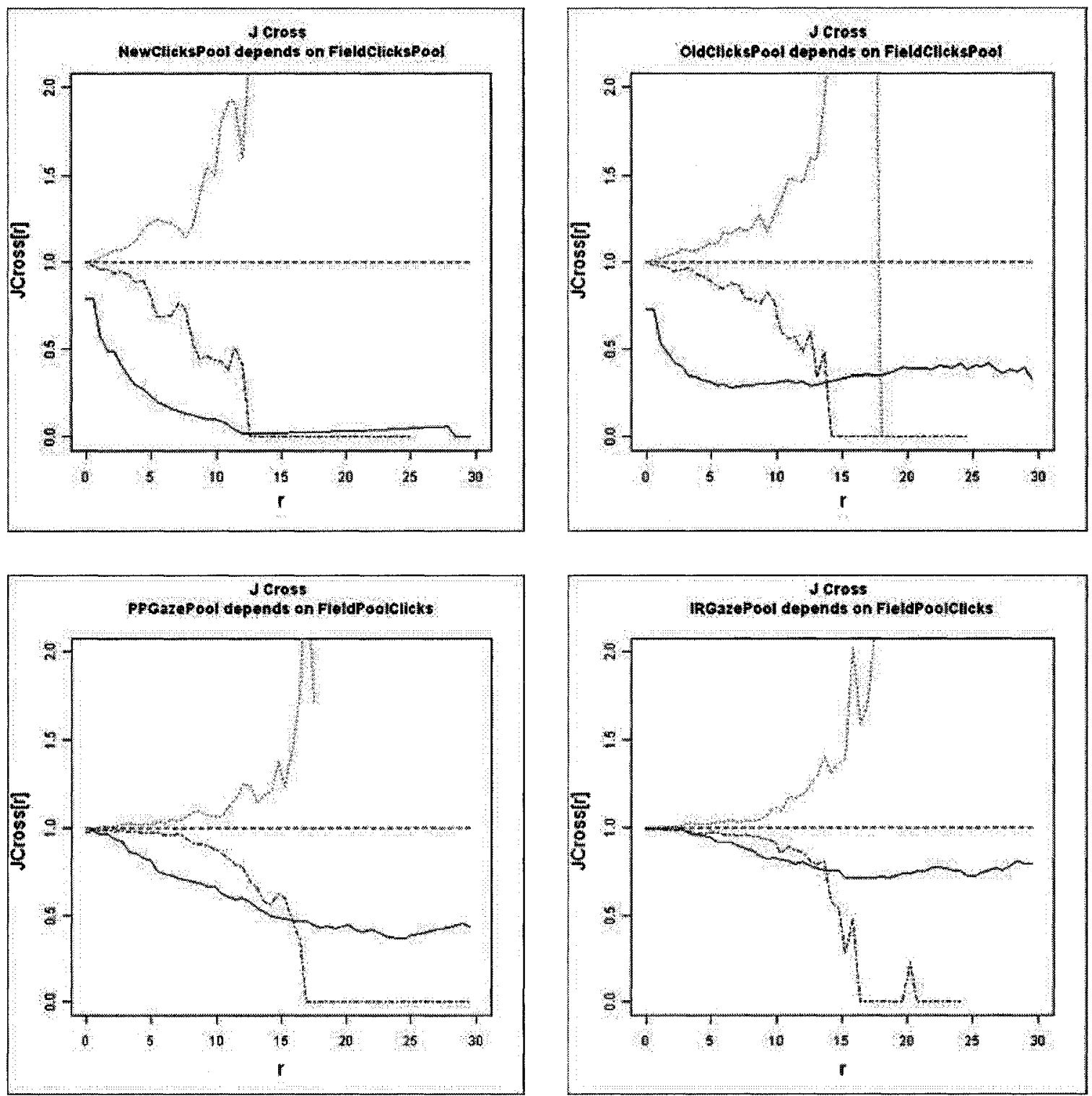

Figure 67. JCross graphs based on similarities between the Pool image new lab click-points data (topleft), old lab click-points data (top-right), PassPoints gaze data (bottom-left), and Image Recall gaze data (bottom-right), when compared to the Pool field click-points data.

\section{Hypothesis 3 Summary: JCross Patterns for Old Clicks and Field Clicks}

Much like the JCross comparisons based on the new lab study click-point data, we can see that the comparisons between the gaze data distributions and the old lab study click-point data are significant, using the Monte Carlo technique. Only one image, 
Paperclips, was not found to be significant at the $9^{\text {th }}$ to $11^{\text {th }}$ radius point in its pattern comparisons. In almost all cases, the new click-point data most resembled the old clickpoint data, as can be seen by the lines being quickly much closer to zero than those of the gaze data sets. The data in the Table 22 represents the randomness of the JCross patterns for both the PassPoints and Image Recall gaze distribution data, as well as the new lab study click-point data, plotted against the old lab study click-point data at a radius of $r=$ 10. Figure 68 demonstrates these results in the form of a bar graph. Table 23 shows a summary of the comparisons.

Table 22. The randomness of the JCross patterns for both PassPoints and Image Recall gaze data, plotted against the new lab study click-point data at a radius of $r=10$.

\begin{tabular}{l|ccc}
\hline \multicolumn{1}{c|}{ Image } & New Clicks & PassPoints & Image Recall \\
\hline Cars & 0.646 & 0.956 & 0.914 \\
Pool & 0.258 & 0.751 & 0.894 \\
Truck & 0.164 & 0.747 & 0.797 \\
Statue & 0.156 & 0.760 & 0.829 \\
Paperclips & 0.977 & 1.005 & 0.943 \\
\hline
\end{tabular}




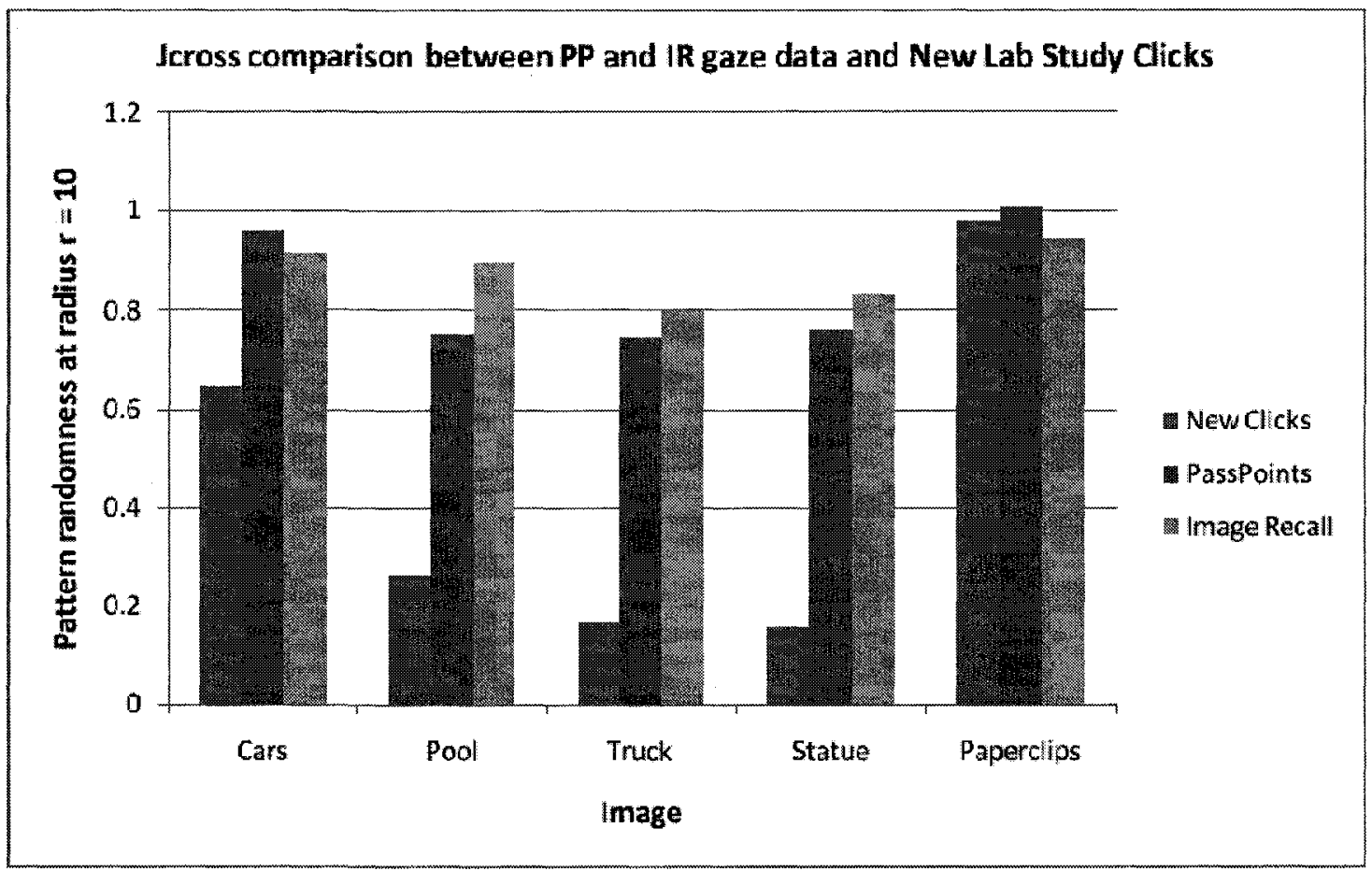

Figure 68. The randomness of the JCross patterns for both PassPoints and Image Recall gaze data, plotted against the new lab study click-point data at a radius of $r=10$.

Table 23. Comparison of analytical results for the JCross Analysis section of Hypothesis 3.

The * indicates that there is no data for the particular item in the table.

Comparison of Analytical Results

JCross Analysis

\begin{tabular}{llll} 
Target: & New Clicks & Old Clicks & \\
Best Attack: & Of Gaze Patterns & Of Gaze Patterns & Overall \\
\hline Cars & PP & IR & Clicks \\
Pool & PP & PP & Clicks \\
Statue & PP & PP & Clicks \\
Truck & PP & PP & Clicks \\
PaperClips & PP & Similar & Similar \\
TopDown & PP & $*$ & $*$ \\
FigureGround & PP & $*$ & $*$ \\
Noise & PP & $*$ & $*$ \\
\hline
\end{tabular}




\section{Hypothesis 3 Overall Summary}

Hypothesis 3 was: "Fixation point patterns resemble click patterns over the same picture across subjects". Each of the three distinct analyses we used in our data analysis provided evidence to support our hypothesis.

We could see from the image analysis that the gaze data from both the PassPoints and the Image Recall conditions overlap the new lab study click-points, the old lab study click-points, and the field study click-points, to some degree depending on the particular image in question. As noted in previous sections, some of the images (Pool and Figureground) seemed to resemble the click-point heatmap regions better than others; the heatmaps for the Paperclips and Noise images were the least comparable.

The results of our dictionary attack analysis showed that each of the attacks was more successful than we would expect by chance, with the exceptions of those on the Paperclips and Noise images. The PassPoints gaze distribution data was better than the Image Recall gaze data, in all cases but one (the Statue image), in predicting the clickpoints of the new lab study data. In attacking the old lab study click-point data, it appeared that the PassPoints gaze data was a better match than the Image Recall gaze data, and that the new click-point data was better than either. However, our ANOVA analysis showed no significant differences between the conditions.

The JCross pattern comparisons demonstrated that, in almost every instance, the new study click-points were significantly similar to the PassPoints and Image Recall gaze distributions. We can see this by looking at the Monte Carlo lines drawn on each of the JCross graphs. Also, in almost every instance, the new lab study click-point data and the gaze data from both conditions were each significantly similar to the old lab study click- 
point data or the field study click-point data (depending which was being used as the comparison model). In the JCross comparisons, the Paperclips and Noise images were again the exceptions.

What we can take away from these analyses collectively is that they provide evidence to support the hypothesis that gaze data resembles graphical password clickpoints data. In particular, the JCross analysis shows that the point-patterns are more similar to each other than would be expected by chance. We note however, that some images seemed to be exceptions, Paperclips and Noise, and for these images the gaze distribution does not appear to overlap the click-points distribution well.

\section{Discussion}

We found little evidence to support our hypotheses $1 \mathrm{~A}$ and 1B, and 2A and 2B. We did find evidence to support hypothesis 3 . We turn our attention to each of the hypotheses, to interpret the results we observed.

\section{Hypotheses 1 and 2}

In dealing with our first hypothesis, we noticed that there was no strong evidence for the existence of left to right or top to bottom trends for the gaze distribution data. In previous research conducted by Chiasson et al. (2008), they had indeed found these trends among participants; however, the trend found among participants was based on their click-points data. In our study, we were interested to see whether these patterns repeated themselves on gaze distribution data. This, apparently, is not the case. Our data indicates that participants looked at various places in the image, in no obvious order, 
when either selecting a graphical password (the PassPoints condition) or when asked to remember an image presented to them on the screen (the Image Recall condition). The participants did not gaze in such a way as to define the left to right or top to bottom trends seen for click-points data in the study by Chiasson et al. In the case of the clickpoints data from that previous study, we speculate that their participants may indeed have looked at the images much in the same way that our participants did. We suggest that the visual search structure used to decide on graphical passwords is different from the eventual pattern selected for the graphical password click-points. We expect that users probably surveyed the image first and then decided where to click.

The second hypothesis dealt with the number of fixations, as well as their dwell times, required for each participant to complete a trial. We had hypothesized that dwell times and the number of fixations would decrease as the trial number got larger. This would be due to the possibility that participants get used to the task of looking at each image in succession, and creating their graphical password — or recalling the image depending on the condition they had been assigned to. However, as we can see from the graphs and statistical analysis, we have no evidence that this occurred. Our results indicated that there were no significant reductions in the dwell times for each successive trial, nor were there reductions in the number of fixations per each successive trial. There could be a number of possible explanations for this outcome. One possibility is that participants in the Image Recall condition were given a set amount of time to recall an image (30 seconds per phase), and they never developed any particular strategy for memorization because it would not have reduced the time required to complete the task; hence, they would continue to fixate and dwell on different parts of each image, in the 
hopes of remembering details better for each successive trial. As for the explanation of the PassPoints condition, we speculate that the participants would have gotten used to the task of selecting click-points to comprise their graphical passwords, but that the number of trials was too few to make this effect observable. More research would be required to explore this.

\section{Hypothesis 3}

In our third hypothesis, we wanted to determine whether participants' gaze distributions would closely resemble click-points data. We tested this three ways, which ended up telling us that the gaze distributions do in fact overlap the click-points data. The gaze distributions from both conditions did resemble click-point distributions more than could be expected by chance. The PassPoints gaze distribution was in almost all cases better than the Image Recall gaze distribution in matching the click-points pattern from our study itself. This can be seen by referring to Tables 19 and 23. We would expect this because the PassPoints gaze data and the new click data come from exactly the same trials, and click-point selection is a visually guided task.

In comparing the PassPoints gaze data and the Image Recall gaze data, as well as the new click data, against the old click-point data, the comparison is more difficult. In our dictionary attack analysis, the graphs suggested that typically the PassPoints gaze data was more effective than the Image Recall gaze data, and that the new click-point data was more effective than either. This seemed plausible because there is greater precision associated with click-points than with gaze fixations. However, our ANOVA analysis demonstrated that there were no significant differences between the three groups. 
In the JCross analysis, using the Monte Carlo technique, we showed that each condition's gaze data matched both the new click-point data and the old click-point data more than could be expected by chance. This was also true for the new click-point data matching the old click-point data. The PassPoints gaze data is consistently better than the Image Recall gaze data. The click-point data from our own study was a better match for the earlier click-point data than was the case for the gaze distribution data for either of our conditions. There were exceptions for the Paperclips and Noise images, which we will discuss later.

This suggests that, if attackers want to create attack dictionaries in order to guess people's click-points, there is evidence that they would benefit from using people's gaze data to create these dictionaries. We have no evidence that gathering the gaze data using a PassPoints system would provide better results than gathering the gaze data using an Image Recall condition. We therefore suggest that attackers would be more likely to gather the gaze data from an Image Recall condition because the system is more passive and requires no physical user interaction. For example, images could be shown in a shop window and eye gaze distribution could be gathered from passersby without their awareness. The JCross data does suggest that a human-seeded attack based on clickpoints would be much better; however, gathering such click data would be a far more difficult process for attackers.

It may be possible to increase the effectiveness of an attack based on gaze data. For example, we must consider that, in our dictionary attack analysis, we only used one specific algorithm based on the technique of Thorpe \& van Oorschot (2007). There may 
be other algorithms for constructing attack dictionaries which may be even more effective.

It might also be possible to gather more effective gaze data. It is feasible that the create phase in the Image Recall task lasted too long. We may have benefitted more from asking participants to look at images for shorter periods of time, which would have emphasized only the strongest points of visual attention. This might have narrowed the scope of the gaze distributions and helped make the dictionary attacks stronger and more accurate. In the PassPoints condition, the gaze data may have been less effective than possible because the participants may have been searching too long for the perfect clickpoints to use as their graphical passwords. Looking at and dwelling on several spots for each image may have created "fake" hotspots, where many participants looked at the points, but none or very few actually clicked on them as part of their graphical passwords.

It might also be possible to improve the fixation data determined by the eye tracker. Our gaze distributions might not match the click-point patterns as well as possible due to the size of the radii which define our click-point and gaze-point hotspots. We opted to use the default settings of the Tobii eye tracker fixation dispersion algorithm. If we were to calculate the fixation points differently in future research, this might possibly change our findings.

\section{Hypothesis 3: Image Differences}

Another point of discussion concerns how click-point selection relates to the complexity of the images used in the study. As was demonstrated by our analysis relating 
to hypothesis 3 , some of the matches between gaze data and click-point data were stronger for some images than for other images. Our results provided little evidence to support the third hypothesis for the Paperclips and Noise images. This could be because they are images that are too repetitive, and users will likely have a difficult time deciding, and thus remembering, their click-points. Different users might therefore select very different points, making them harder to guess. This might result in different users' clickpoint patterns and gaze patterns being essentially unrelated, which is suggested by the JCross graphs. This would make click-points on these images difficult for attackers to guess. However, it would also likely make the click-points difficult for users to remember. The Paperclips and Noise images were associated with the longest create time phases in the PassPoints condition. This would indicate that these particular images are unlikely candidates to be actually used in graphical password systems.

Recent research conducted by Salehi-Abari, Thorpe, \& van Oorschot (2008), used the images and click-points data from the lab study reported by Chiasson et al. (2007), and calculated the complexity of each of the images used in the study. They did this with the use of an algorithm which broke down the images, gauged and calculated the number of centers as well as the number of corners contained in each image, giving 2 numbers for each image making up the image complexity for any of the particular images. The centers indicate the number of distinct objects in an image, and the corners would also reflect the complexity of the objects. For our study, we used five of the same images, as well as three extra images which we created on our own. We obtained their data relating to the image complexity for each of the 5 images, as well as our extra 3 images. The data can be found below in Table 24. A bar graph of the data can be found in Figure 69. 
Table 24. Image complexity calculations. Includes the number of centers and the number of corners in each of the 8 images used.

\begin{tabular}{l|cc}
\hline \multicolumn{1}{c|}{ Image } & \# centers & \# corners \\
\hline Pool image & 431 & 337 \\
Cars image & 667 & 397 \\
Truck image & 699 & 422 \\
Paperclips image & 645 & 408 \\
Statue image & 285 & 236 \\
Figureground image & 22 & 71 \\
Topdown image & 89 & 103 \\
Noise image & 1041 & 518 \\
\hline
\end{tabular}

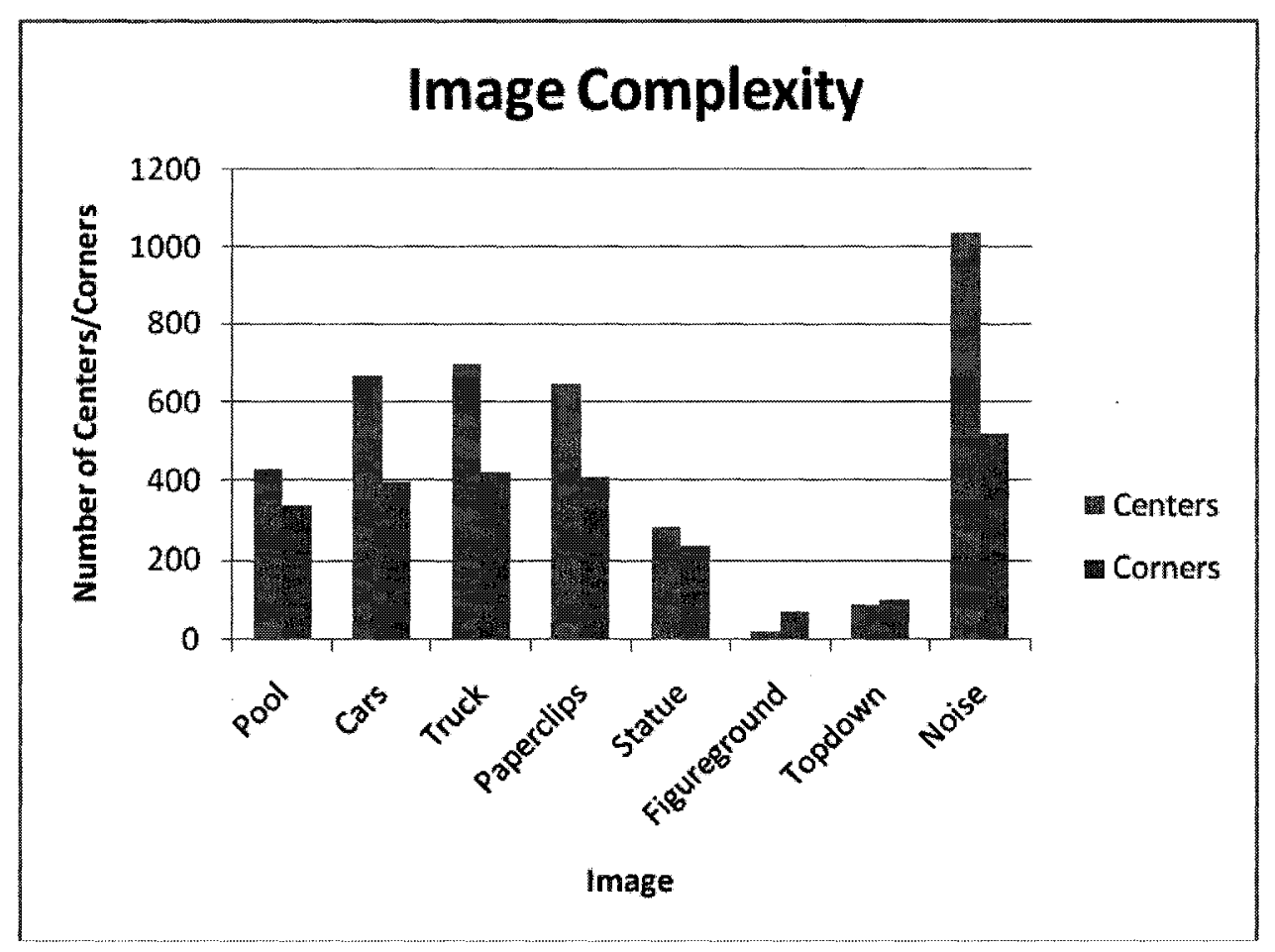

Figure 69. Image complexity calculations.

Relating the resulting data from our study to the complexity calculations of each image suggests that these calculations may not be consistent indicators of the best or worst images for usability or security. Our dictionary attacks on the Paperclips image 
demonstrated that users selected click-points in different patterns from one another, since it was apparent that the paperclips depicted in the image were placed in random sections of the image with no discernible pattern. We speculated that such an image would undoubtedly be categorized within a higher degree of centers and corners, thus making it more complex as per our colleagues' calculations. However, as we can see from Figure 69, the Paperclips image shares a similar complexity calculation with the Truck and Cars images, which were much more susceptible to our dictionary attacks. Conversely, the Figureground and Topdown images were rated as much less complex than the other images, but the gaze patterns matched the click patterns well, leading to effective attacks. As such, we can speculate that this measure of complexity plays little to no importance in the degree of difficulty of click-point pattern selection in the creation of graphical passwords, nor in the effectiveness of dictionary attacks on low or highly complex images. We speculate that a more sophisticated understanding of complexity, involving salience, is needed for further understanding of this issue.

The three new images that were created for our particular experimental study, Figureground, Topdown, and Noise, ranged from slightly complex to highly complex. As such, we would have expected that attacks on the highly complex image (Noise) would have yielded a much smaller cumulative ratio of hits than the attacks on the less complex images (Figureground and Topdown). This was not the case. The attacks on the Noise and Figureground images were better in predicting click-points than were the attacks on the Topdown image, despite Noise being much more complex than Topdown. The visual processing of the images by the user would have varied for each individual image, given their complexity. Participants may have approached each image using a particular 
visually guided task, from either a top-down or a bottom-up visual process, depending on the complexity of the image itself. It seems that the images that were difficult to guess (and possibly remember) were those where there was similarity across the whole image and no salient features anywhere. For these particular images, we speculate participants maintained a bottom-up visual search strategy and did not consistently find similar places on the image to click. We know that participants in the PassPoints condition selected click-points in the four corners and in the center of the image, while they selected the only red rectangle more often than any other rectangle in the Topdown image.

\section{Conclusion}

Graphical password schemes, such as the PassPoints scheme, are a new and promising approach to authentication. The argument for the use of this new type of password was the memorability of images and the likelihood of more random and therefore more secure passwords. Research on the usability of graphical passwords, in particular those studies conducted independently by Wiedenbeck et al. (2005) and Chiasson et al. (2007), showed that this type of graphical passwords offered good usability over text-based passwords, with a few limitations. Other research, on the security of PassPoints passwords, conducted by Birget (2006) and Thorpe et al. (2007), looked at various attack strategies. Thorpe et al. showed that PassPoints passwords, although characterized by a potential for high theoretical password spaces, demonstrated some security problems related to concentrations in user choice of click-points. From the results obtained in their experiments, we developed the notion of capturing gaze data to 
create attack dictionaries in order to see if gaze data could in fact predict individual clickpoints.

Our study method involved participants in one of two conditions: the creation of graphical passwords (PassPoints condition), and the recalling of images (Image Recall condition), both of which required the participant's gaze data to be recorded using a Tobii eye tracker. We identified the main hypotheses to explore our main research question. The first hypothesis was divided into two parts, and dealt with the possibility of the existence of trends among the data; the second hypothesis was also divided into two parts, which dealt with the possibility of a reduction of fixation and dwell times within the gaze data over successive trials; finally, the third hypothesis dealt with the bulk of the main research question, which was whether gaze distribution statistics overlapped clickpoints data on the same images used in both experimental conditions.

Upon analysis of hypothesis 1 , it was found that there was no significant evidence for the existence of horizontal or vertical trends among the gaze distribution data. It was found for hypothesis 2 that, as each successive trial was completed for each participant, we saw no evidence that the number of fixations and dwell times decreased. More research is required to understand such possible patterns in gaze data.

Several steps were taken in order to test the third hypothesis. These included three distinct analyses. With the first analysis, a comparison of gaze fixation and click-point patterns overlaid on top of their original image, we found that the gaze data distribution patterns resembled the click-point data patterns. The second analysis consisted of dictionary attacks of the gaze data on the new click data, and each of those data sets on the old lab study data sets of Chiasson et al. (2007), as well as the field study data (this 
data was available for two images only). Both the PassPoints and Image Recall gaze data were effective in guessing the new click-point data, but the PassPoints data was almost always more effective. Also, both sets of gaze data and the new click-point data were more effective than chance in guessing the older study data. An analysis of variance was conducted on the effect of the three conditions of the dictionary attack on the click-points from the older lab study, with four levels of dictionary size. The result of the analysis of variance relating to the attack conditions was inconclusive, suggesting no evidence for the superiority of any of the three attack strategies.

The last analysis on the data dealt with a statistic, which is of particular use when attempting to make sense of two overlapping point patterns, the JCross statistic. Upon analysis of the JCross statistic, and a Monte Carlo analysis on each on each of the images used in our study, it was determined that each set of gaze data was significantly similar to the new click-point data when using a radius of 9 to 11 pixels. Also, each of the gaze distribution patterns, as well as the new click-point data patterns, significantly overlapped the old click-point patterns.

There were, however, some images for which the findings above were not the case, which may indicate that some images lead to gaze distribution data that does not closely match click-point data. We speculate that images that are repetitive and without salient features lead to gaze distributions and click-point patterns which are unrelated to the image.

The research question for this thesis was: "can preselection gaze distribution statistics predict graphical passwords?" We narrowed our study and addressed the issue of whether gaze distribution can predict individual click-points in PassPoints graphical 
passwords. Based on the results from the dictionary attacks, the comparisons between JCross graphs for each of the conditions in our study, and the statistical analyses conducted on our data sets, we come to the conclusion that gaze distribution data can help in predicting to some degree likely click-points comprising graphical passwords. This is alarming because there is no physical interaction required to acquire gaze data; this is therefore a potentially dangerous attack strategy. Work by Thorpe and van Oorschot (2007) and Dirik et al. (2007) have shown that hotspots emerging from user choice pose a threat to the security of the PassPoints scheme. Our contribution is to show that attack dictionaries can be constructed based on eye gaze information alone. Note, however, that our work addressed the guessing of individual click-points rather than entire passwords. This suggests that, with eye tracking technology, effective attack dictionaries might be built automatically by displaying images in a public place and gathering human gaze data without the need for physical interaction or awareness.

In terms of future work, our attack analysis should be extended to support attacking whole passwords, and not only individual click-points. We could also create and test new algorithms to use in dictionary attacks on click-points that may yield stronger results than were obtained using our current methods of attack. Also, we speculate that using a different radius to calculate fixation point patterns may increase the likelihood of obtaining more meaningful gaze distribution data from our participants. We further suggest more research is needed on how particular images and salient details affect user choice of click-points in graphical passwords. 


\section{References Cited}

Adams , A., Sasse, M. A., \& Lunt, P. (1997). Making Passwords Secure and Usable, Proceedings of HCI on People and Computers XII, p.1-19, January 1997.

Baddeley, A., \& Turner, R. (2005). An R package for analyzing spatial point patterns. Journal of Statistical Software, 12(6).

Birget, J.C., Hong, D., \& Memon, N. Graphical Passwords Based on Robust Discretization. IEEE Transactions on Information Forensics and Security 1(3), pp. 395-399, 2006.

Blonder, G.E. (1996). Graphical passwords. Lucent Technologies, Inc., Murray Hill, NJ, U. S. Patent, Ed. United States, 1996.

Brostoff, S, \& Sasse, M. A. (2000). Are Passfaces more usable than passwords? A field trial investigation. In S. McDonald, Y. Waern, \& G. Cockton (Eds), People and Computers IX-Usability or Else! Proceedings of HCI 2000, 405-424, Springer.

Chiasson, S., Biddle, R., \& van Oorschot, P.C. (2007). A second look at the usability of click-based graphical passwords. Symposium on Usable Privacy and Security (SOUPS), 1-12.

Chiasson, S., Forget, A., Biddle, R., \& van Oorschot, P. C. (2008). User interface design affects security: patterns in click-based graphical passwords. Technical Report TR-08-14, Carleton University, Ottawa.

Chun, M. M., \& Wolfe, J. M. (2000). Visual Attention. Blackwell Handbook of Perception. E.B. Goldstein. 
Dhamija, R., \& Perrig, A. (2000). Déjà vu: a user study using images for authentication. USENIX Security Symposium, Proceedings of the $9^{\text {th }}$ conference on USENLX Security Symposium.

De Luca, A., Weiss, R., \& Drewes, H. (2007). Evaluation of eye-gaze interaction methods for security enhanced PIN-entry. OzCHI 2007 Proceedings, 199-202.

Dirik, A. E., Memon, N., \& Birget, J. C. (2007). Modeling user choice in the PassPoints graphical password scheme. Symposium On Usable Privacy and Security (SOUPS), technical paper.

Duchowski, A. (2007). Eye Tracking Methodology: Theory and Practice. Springer.

Folk, C. L., \& Remington, R. W. (2008). Bottom-up priming of top-down attentional control settings. Visual Cognition, 16(2/3), 215-231.

Gold, J. M., Murray, R. F., Sekuler, A. B., Bennet, P. J., \& Sekuler, R. (2005). Visual memory decay is deterministic. Psychological Science, 16(10), 769-774.

Government of Ontario, Health and Safety Guidelines (2004). Computer ergonomics: workstation layout and lighting. Queen's Printer for Ontario.

Jermyn, I., Mayer, A., Monrose, F., Reiter, M., \& Rubin, A. (1999). The Design and Analysis of Graphical Passwords. USENLX Security Symposium, Proceedings of the $8^{\text {th }}$ conference on USENIX Security Symposium.

Karacan, H. U., \& Hayhoe, M. M. (2008). Is attention drawn to changes in familiar scenes? Visual Cognition, 16(2/3), 356-374.

Koike, T., \& Saiki, J. (2006). Stochastic saliency-based search model for search asymmetry with uncertain targets. Journal of Neurocomputing, 69, 2112-2126. 
Kumar, M., Garfinkel, T., Boneh, D., \& Winograd, T. (2007). Reducing shoulder-surfing by using gaze-based password entry. Symposium On Usable Privacy and Security (SOUPS), 13-19.

Land, M.F., \& Lee, D.N. (1994). Where we look when we steer. Nature, 369, 742-744.

Menezes, A.,van Oorschot, P., \& Vanstone, S. (1996). Handbook of Applied Cryptography, CRC Press, 385-424.

Monrose, F., and Reiter, M. (2005). Graphical passwords. In L. Cranor and S. Garfinkel, editors, Security and Usability: Designing Secure Systems That People Can Use, Chapter 9, pages 157-174. OReilley.

Muller, H. J., \& Krummenacher, J. (2006). Visual search and selective attention. Visual Cognition, 14 (4/5/6/7/8), 389-410.

Openwall Project. John the Ripper password cracker. http://www.openwall.com/john/, site accessed March 6th, 2008.

Peters, M. (1995). Revised Vandenberg \& Kuse mental rotations tests: forms MRT-A to MRT-D. Technical Report, Department of Psychology, University of Guelph.

Rayner, K. (1995). Eye movements and cognitive processes in reading, visual search, and scene perception. In J.M. Findlay, R. Walker, \& R.W. Kentridge (Eds), Eye Movements Research: Mechanisms, Processes, and Applications, 3-21. New York: Elsevier.

Salehi-Abari, A., Thorpe, J., \& van Oorschot, P. C. (2008). On purely automated attacks and click-based graphical passwords. $24^{\text {th }}$ Annual Computer Security Applications Conference. 
Salvucci, D.D., \& Goldberg, J.H. (2000). Identifying fixations and saccades in eyetracking protocols. Eye Tracking Research \& Applications Symposium 2000, 7178.

Shao, L., \& Brady, M. (2006). Specific object retrieval based on salient regions. Pattern Recognition, 39, 1932-1948.

Sperling, G. (1960). The information available in brief visual presentations. Psychological Monographs : General and Applied, 74, 1-29.

Suo, X, Zhu, Y., \& Owen, G.S. (2005). Graphical passwords: a survey. Annual Computer Security Applications Conference.

Theeuwes, J., \& van der Burg, E. (2008). The role of cueing in attentional capture. Visual Cognition, 16(2/3), 232-247.

Thorpe, J. \& van Oorschot, P.C. (2007). Human-seeded attacks and exploiting hot-spots in graphical passwords. USENIX Security Symposium, Proceedings of the $16^{\text {th }}$ conference on USENIX Security Symposium.

Tobii Technology AB. (2003). Tobii 1750 Eye-tracker. Danderyd, Sweden.

Tobii Technology AB. (2006). Tobii Eye Tracker and ClearView Analysis Software Manual. Danderyd, Sweden.

van Lieshout, M.N.M. \& Baddeley, A. J. (1996). A non-parametric measure of spatial interaction in point patterns. Statistica Neerlandica, 50(3), 344-361.

van Lieshout, M.N.M. \& Baddeley, A. J. (1999). Indices of dependence between types in multivariate point patters. Scandinavian Journal of Statistics, 26, 511-532.

van Oorschot, P.C., \& Thorpe, J. (2008). On predictive models and user-drawn graphical passwords. ACM TISSEC, 10(4). 
Wiedenbeck, S., Waters, J., Birget, J-C., Brodskiy, A., \& Memon, N. (2005). PassPoints: design and longitudinal evaluation of a graphical password system. International Journal of Human-Computer Studies, 63, 102-127.

Wolfe, J. M., Horowitz, T. S. \& Michod, K. O. (2007). Is visual attention required for robust picture memory? Journal of Vision Research, 47, 955-964. 


\section{Appendices}

\section{Appendix A: Ethics Proposal}

\section{Study Proposal}

The proposed research seeks to investigate the ease of use, security, and effectiveness of graphical passwords. A graphical password is created by clicking on a set of points on an image. Users then repeat this sequence of clicks whenever they need to re-enter their password. The goal of this research is twofold. First we want to investigate what points users tend to select as part of graphical passwords. The investigation will involve showing a series of images to users and asking them to pick a password for each. We want to see whether there are patterns in user choices and whether image characteristics (such as photographs versus cartoons, or the amount of visual clutter) affect choices. From a computer security perspective, we want to determine whether graphical passwords are likely to be secure and difficult for others to guess. Secondly, we want to investigate whether people's eye gaze has an impact on the security of graphical passwords. In other words, we want to see whether we can guess where people click on an image by knowing where they look on that same image. As such, a Tobii eye tracker will be used in order to gather gaze fixation data for each participant.

Formal usability tests will be conducted to test different aspects of graphical passwords. The tests are planned to uncover usability problems and test the effectiveness of various design decisions such as image choice.

This proposal concerns the protocol regarding the usability tests. The primary focus of the usability studies is to assess the effectiveness of graphical passwords as alternatives to text-based passwords for systems requiring user authentication. The usability test will also examine the perceptions and preferences of the users and will prompt the users for suggestions for improvements to the interfaces. 


\section{Method}

The study consists of a usability test that requires the recruitment of participants. The procedure and materials are described in the following sections and included in the Appendices. Participants are asked to complete a one-hour session which will be used to test specific aspects of the interface such as what points users select as passwords for different images.

\section{Usability Test}

Participants. A potential user group has been identified as typical target users. This group consists of computer users who are familiar with navigating the web and with entering passwords to $\log$ on to computer systems. Participants will be primarily recruited from the University community. Up to 150 participants will be recruited in total to take part in the different usability tests. Participants will be paid a $\$ 20$ honorarium ( $\$ 10$ for participants recruited via the Carleton SONA system), or given course credit, for their time. A notice of recruitment will be posted on bulletin boards around campus. Some of the participants for this study will be recruited through the Psychology's Online Experiment Recruitment System (SONA). This same notice may be handed out to some personal contacts that would likely be willing to participate in the study.

Materials. The materials include: a computer with internet access and the experiment software installed, a Tobii eye tracker, an informed consent form (See Appendix B), a participant information form (See Appendix D), a set of instructions (See Appendix E), usability task descriptions (See Appendix F for a sample), a Post-task 
questionnaire (See Appendix G), a debriefing form (See Appendix H) and a pencil or pen.

Procedure. Participants will be recruited via a notice posted on bulletin boards at Carleton University, or through the Psychology Department's online recruiting system (SONA). After being escorted to the experiment room, participants will be given an informed consent form to read over and sign, if they wish to take part in the study. Each participant will be informed that the usability test is to test the software and not them. They will be reminded that they are not to feel bad if they cannot complete every task because the tasks are designed to test how easy the software is to use. They will also be reminded that they can quit at any time during the session. Talk aloud protocol will be used, which requires participants to verbalize what they are thinking when they are attempting to complete each task.

The testing will include an eye tracker. The eye tracker is incorporated into the computer screen and participants use the computer as they would normally. The eye tracker only records a map of where a person is looking on the computer screen; it is not a camera that records the user's face or eyes.

Participants will be presented with a series of images. The selected images will be non-offensive; for example they may consist of landscapes, scenes from a public area (like a park or busy street), or pictures of everyday items (like a display of pottery items). We will also insert a few pictures that are made up of either random patterns (noise), or images with no discernible pattern (e.g. all one colour). There will be two conditions for testing. 
Condition 1. The participants in this condition will create passwords by clicking on points in the aforementioned images. Participants will be asked to create their password to ensure that they select realistic points that they can repeat. An initial training phase will help participants become familiar with the idea of entering passwords in this manner. During training, they will be given an image (or sequence of images), asked to pick a password, and then re-enter this same password. They will be given feedback indicating that they were successful or provided with help to correct any problems.

Next, participants will be given a new image and asked to pretend that they are choosing a real password to protect their private information (such as their banking information). They will confirm this password by re-entering it. A distraction task will be administered to change their focus for 30-60 seconds. The distraction task will be visual/spatial in nature, and is done by completing a question from the Mental Rotations Test. Participants will then be asked to re-enter their password. This process will be repeated for a series of trials (the exact number will be determined through pilot testing, but we estimate that it will be approximately 10). The system will record the user's mouse clicks so that the passwords can be later analyzed for patterns in the users' choices. Since the passwords are unlike any real passwords that the participants have and since our passwords will not be used to protect any private information, analysis of the password choices does not present any privacy or security concerns.

Participants will be asked to complete a post-test questionnaire after the session. It will request their opinion of graphical passwords through Likert-scale and open-ended questions. Prior to being debriefed, participants will be asked to provide suggestions on 
what could be done to improve the software. Participants will be debriefed at the conclusion of the usability test.

Condition 2. In this condition, participants will be asked to view the same images as the participants in the first condition; however, they will not be asked to create any graphical passwords. Instead, they will simply be asked to look at each image and prepare for later recall questions, for a total of 45 seconds. Before being asked to recall details on each image, they will be given the same distraction task as the condition 1 participants. This Mental Rotation Task lasts approximately 30 seconds.

We are interested in looking at whether the gaze data is any different from the participants who are asked to view the same images, and create graphical passwords. These participants, contrary to condition 1 , will later be asked to recall the images they had seen previously. This is done in order to better understand people's gaze patterns when presented with visual stimuli on a computer screen. We are hoping to differentiate the gaze patterns from this condition to the first condition.

The Tobii eye tracker will record where users gazed so that we can analyze where they looked while being shown each image.

Participants will be asked to complete a post-test questionnaire after the session. This post-task questionnaire will ask each participant's opinion on the images they saw, what they were looking at/for when visually scanning each image. They will also be asked what they thought of the difficulty associated with recalling each image based on the visual patterns present within each image (e.g. one-colour images or noise, as opposed to images depicting real-life scenes). 
For both conditions, time limits will not be imposed, but the tasks will be designed to one hour at most. They will be told that they are free to give up when they feel they have spent enough time on the task and they will be informed that they are welcome to take rest breaks throughout the study. 


\section{Appendix B: Announcement for Recruitment - Usability Test}

Participants will be recruited in three ways:

\section{Approaching Prospective Participants}

Prospective participants will be approached and asked if they would be willing to participate in the usability test.

\section{Posting a Notice of Recruitment}

Notices of recruitment, requesting participation in the usability test, will be posted on bulletin boards at Carleton University. Such postings will carry the following message:

Hello,

The HotLab conducts research on human-computer interaction and usability. We are currently conducting a study on the usability of graphical passwords. Graphical passwords are entered by clicking on pre-determined points of an image. The goal is to gain some insight on the practicality of graphical passwords, determine what types of images lead to the best passwords, and evaluate the usability of graphical passwords.

We are recruiting volunteers to take part in the usability test. Participants should have experience browsing the web and logging on to computer accounts using a password. Participants will have the choice between receiving a $\$ 20.00$ honorarium or a $1.0 \%$ course credit in either PSYC 1001 or PSYC 1002.

If you are interested in participating in this project, please contact us at:

dblanc@connect.carleton.ca

Kind Regards

Daniel LeBlanc

M.A. Candidate in Psychology

HotLab, Carleton University

Posting a Notice on the Psychology Department's Online Recruitment System (SONA)

A notice of recruitment will be posted through the Psychology Department's Online Recruitment System.

Posted notice of recruitment will contain the following information:

- We are conducting a study on the usability and security of graphical passwords

- We are recruiting volunteers to take part in a usability and security test

- Participants will receive a $\$ 10.00$ honorarium or a $1.0 \%$ course credit in either PSYC 1001 or PSYC 1002

- For more information, please send an email to: dblanc@aconnect.carleton.ca 


\section{Appendix C: Consent Form-Usability Test-Graphical Passwords}

\section{Research Personnel}

\begin{tabular}{|l|l|}
\hline Daniel LeBlanc & Dr. Robert Biddle \\
Principle Investigator & Faculty Sponsor \\
Carleton University & Carleton University \\
$(613) 435-1290$ & (613) 520-2600 Ext. 6317 \\
dblanc@.connect.carleton.ca & robert_biddle@carleton.ca \\
\hline
\end{tabular}

Purpose

The purpose of this usability test is to evaluate the usability, security, and effectiveness of graphical passwords. We are trying to determine whether graphical passwords are a viable alternative to regular passwords. An eye tracker will be detecting eye movements during the session.

\section{Task Requirements}

We will ask you to complete a series of tasks that involve choosing and entering graphical passwords. We ask you to think out loud while you work on them. We will then ask that you fill out a short questionnaire, and at the conclusion of the testing session you will be asked to provide us with any suggestions you might have to improve the software.

\section{Duration and Locale}

Each session should take approximately 1 hour. You will be paid a $\$ 20$ honorarium, or given course credit, for your time. The testing will primarily take place at the HotLab located in room 210 SSRB.

\section{Potential Risk/Discomfort}

There will be no psychological or physical risk.

\section{Anonymity/Confidentiality}

All data that is collected will be held completely confidential. The data will only be made available to those people involved with this testing. Data will be coded for identification purposes.

\section{Right to Withdraw}

You have the right to withdraw at any time, without any explanation as to the reason for withdrawing from the testing. You will receive the $\$ 20$ honorarium, or course credit, even if you choose to withdraw from the study.

\section{Ethics Approval}

This study has been approved by the ethics committee for psychology research. 
If you have concerns about the ethics of this research, please contact Dr. Avi Parush. For other questions about the research, please contact Dr. Anne Bowker:

Dr. Anne Bowker

Chair, Department of Psychology

Carleton University

(613) 5202600 ext. 2648

psychchair@carleton.ca
Dr. Avi Parush

Chair, Carleton University Ethics Committee for Psychological Research

Carleton University

(613) 5202600 ext . 6026

avi_parush@carleton.ca

\section{Signatures}

I have read and understand the above terms of testing and I understand the conditions of my participation. My signature indicates that I agree to participate in this experiment.

Participant's Name:

Participant's Signature:

Researcher's Name:

Researcher's Signature: 
Appendix C: Consent Form - Usability Test-Graphical Passwords - July 1st

Research Personnel

\begin{tabular}{|l|l|}
\hline Daniel LeBlanc & Dr. Robert Biddle \\
Principle Investigator & Faculty Sponsor \\
Carleton University & Carleton University \\
$(613) 435-1290$ & $(613) 520-2600$ Ext. 6317 \\
dblanc@connect.carleton.ca & robert_biddle@carleton.ca \\
\hline
\end{tabular}

Purpose

The purpose of this usability test is to evaluate the usability, security, and effectiveness of graphical passwords. We are trying to determine whether graphical passwords are a viable alternative to regular passwords. An eye tracker will be detecting eye movements during the session.

\section{Task Requirements}

We will ask you to complete a series of tasks that involve choosing and entering graphical passwords. We ask you to think out loud while you work on them. We will then ask that you fill out a short questionnaire, and at the conclusion of the testing session you will be asked to provide us with any suggestions you might have to improve the software.

\section{Duration and Locale}

Each session should take approximately 1 hour. You will be paid a $\$ 20$ honorarium, or given course credit, for your time. The testing will primarily take place at the HotLab located in room 210 SSRB.

\section{Potential Risk/Discomfort}

There will be no psychological or physical risk.

Anonymity/Confidentiality

All data that is collected will be held completely confidential. The data will only be made available to those people involved with this testing. Data will be coded for identification purposes.

\section{Right to Withdraw}

You have the right to withdraw at any time, without any explanation as to the reason for withdrawing from the testing. You will receive the $\$ 20$ honorarium, or course credit, even if you choose to withdraw from the study.

\section{Ethics Approval}

This study has been approved by the ethics committee for psychology research. 
If you have concerns about the ethics of this research, please contact Dr. Avi Parush. For other questions about the research, please contact Dr. Janet Mantler:

\begin{tabular}{|l|l|}
\hline Dr. Janet Mantler & $\begin{array}{l}\text { Dr. Avi Parush } \\
\text { Chair, Carleton University Ethics Committee for } \\
\text { Psychological Research } \\
\text { Carleton University }\end{array}$ \\
Carleton University & (613) 5202600 ext .6026 \\
(613) 5202600 ext. 4173 & avi_parush@carleton.ca \\
psychchair@carleton.ca & \\
\hline
\end{tabular}

\section{Signatures}

I have read and understand the above terms of testing and I understand the conditions of my participation. My signature indicates that I agree to participate in this experiment.

Participant's Name:

Participant's Signature:

Researcher's Name:

Researcher's Signature: 
Appendix C: Consent Form - Usability Test-Graphical Passwords - SONA

Research Personnel

\begin{tabular}{|l|l|}
\hline Daniel LeBlanc & Dr. Robert Biddle \\
Principle Investigator & Faculty Sponsor \\
Carleton University & Carleton University \\
$(613) 435-1290$ & $(613) 520-2600$ Ext. 6317 \\
dblanc@connect.carleton.ca & robert_biddle@carleton.ca \\
\hline
\end{tabular}

Purpose

The purpose of this usability test is to evaluate the usability, security, and effectiveness of graphical passwords. We are trying to determine whether graphical passwords are a viable alternative to regular passwords. An eye tracker will be detecting eye movements during the session.

\section{Task Requirements}

We will ask you to complete a series of tasks that involve choosing and entering graphical passwords. We ask you to think out loud while you work on them. We will then ask that you fill out a short questionnaire, and at the conclusion of the testing session you will be asked to provide us with any suggestions you might have to improve the software.

\section{Duration and Locale}

Each session should take approximately 1 hour. You will be paid a $\$ 10$ honorarium, or given course credit, for your time. The testing will primarily take place at the HotLab located in room 210 SSRB.

\section{Potential Risk/Discomfort}

There will be no psychological or physical risk.

\section{Anonymity/Confidentiality}

All data that is collected will be held completely confidential. The data will only be made available to those people involved with this testing. Data will be coded for identification purposes.

\section{Right to Withdraw}

You have the right to withdraw at any time, without any explanation as to the reason for withdrawing from the testing. You will receive the $\$ 10$ honorarium, or course credit, even if you choose to withdraw from the study.

\section{Ethics Approval}

This study has been approved by the ethics committee for psychology research. 
If you have concerns about the ethics of this research, please contact Dr. Avi Parush. For other questions about the research, please contact Dr. Anne Bowker:

Dr. Anne Bowker

Chair, Department of Psychology

Carleton University

(613) 5202600 ext. 2648

psychchair@carleton.ca
Dr. Avi Parush

Chair, Carleton University Ethics Committee for Psychological Research

Carleton University

(613) 5202600 ext .6026

avi_parush@carleton.ca

\section{Signatures}

I have read and understand the above terms of testing and I understand the conditions of my participation. My signature indicates that I agree to participate in this experiment.

Participant's Name:

Participant's Signature:

Researcher's Name:

Researcher's Signature: 
Appendix C: Consent Form - Usability Test-Graphical Passwords - SONA - July 1st

\section{Research Personnel}

\begin{tabular}{|l|l|}
\hline Daniel LeBlanc & Dr. Robert Biddle \\
Principal Investigator & Faculty Sponsor \\
Carleton University & Carleton University \\
$(613) 435-1290$ & $(613) 520-2600$ Ext. 6317 \\
dblanc@connect.carleton.ca & robert_biddle@carleton.ca \\
\hline
\end{tabular}

Purpose

The purpose of this usability test is to evaluate the usability, security, and effectiveness of graphical passwords. We are trying to determine whether graphical passwords are a viable alternative to regular passwords. An eye tracker will be detecting eye movements during the session.

\section{Task Requirements}

We will ask you to complete a series of tasks that involve choosing and entering graphical passwords. We ask you to think out loud while you work on them. We will then ask that you fill out a short questionnaire, and at the conclusion of the testing session you will be asked to provide us with any suggestions you might have to improve the software.

\section{Duration and Locale}

Each session should take approximately 1 hour. You will be paid a $\$ 10$ honorarium, or given course credit, for your time. The testing will primarily take place at the HotLab located in room 210 SSRB.

\section{Potential Risk/Discomfort}

There will be no psychological or physical risk.

\section{Anonymity/Confidentiality}

All data that is collected will be held completely confidential. The data will only be made available to those people involved with this testing. Data will be coded for identification purposes.

\section{Right to Withdraw}

You have the right to withdraw at any time, without any explanation as to the reason for withdrawing from the testing. You will receive the $\$ 10$ honorarium, or course credit, even if you choose to withdraw from the study.

\section{Ethics Approval}

This study has been approved by the ethics committee for psychology research. 
If you have concerns about the ethics of this research, please contact Dr. Avi Parush. For other questions about the research, please contact Dr. Janet Mantler:

Dr. Janet Mantler

Chair, Department of Psychology

Carleton University

(613) 5202600 ext. 4173

psychchair@carleton.ca
Dr. Avi Parush

Chair, Carleton University Ethics Committee for

Psychological Research

Carleton University

(613) 5202600 ext . 6026

avi_parush@carleton.ca

Signatures

I have read and understand the above terms of testing and I understand the conditions of my participation. My signature indicates that I agree to participate in this experiment.

Participant's Name:

Participant's Signature:

Researcher's Name:

Researcher's Signature: 


\section{Appendix D: Consent Form - Usability Test - Image Memory Test}

\section{Research Personnel}

\begin{tabular}{|l|l|}
\hline Daniel LeBlanc & Dr. Robert Biddle \\
Principle Investigator & Faculty Sponsor \\
Carleton University & Carleton University \\
$(613) 435-1290$ & $(613) 520-2600$ Ext. 6317 \\
dblanc@connect.carleton.ca & robert_biddle@carleton.ca \\
\hline
\end{tabular}

Purpose

The purpose of this usability test is to evaluate the visual patterns associated with images on a computer screen to investigate whether images can be used as passwords. We are trying to determine what patterns result from the characteristics of visual search in visual images. An eye tracker will be detecting eye movements during the session.

\section{Task Requirements}

We will ask you to complete a series of tasks that involve looking at various images on a computer screen for a preset amount of time. We will then ask that you fill out a short questionnaire, and at the conclusion of the testing session you will be asked to provide us with any suggestions you might have to improve the software.

\section{Duration and Locale}

Each session should take approximately 1 hour. You will be paid a $\$ 20$ honorarium, or given course credit, for your time. The testing will primarily take place at the HotLab located in room 210 SSRB.

\section{Potential Risk/Discomfort}

There will be no psychological or physical risk.

\section{Anonymity/Confidentiality}

All data that is collected will be held completely confidential. The data will only be made available to those people involved with this testing. Data will be coded for identification purposes.

\section{Right to Withdraw}

You have the right to withdraw at any time, without any explanation as to the reason for withdrawing from the testing. You will receive the $\$ 20$ honorarium, or course credit, even if you choose to withdraw from the study.

\section{Ethics Approval}

This study has been approved by the ethics committee for psychology research. 
If you have concerns about the ethics of this research, please contact Dr. Avi Parush. For other questions about the research, please contact Dr. Anne Bowker:

Dr. Anne Bowker

Chair, Department of Psychology

Carleton University

(613) 5202600 ext. 2648

psychchair@carleton.ca
Dr. Avi Parush

Chair, Carleton University Ethics Committee for Psychological Research

Carleton University

(613) 5202600 ext . 6026

avi_parush@carleton.ca

\section{Signatures}

I have read and understand the above terms of testing and I understand the conditions of my participation. My signature indicates that I agree to participate in this experiment.

Participant's Name:

Participant's Signature:

Researcher's Name:

Researcher's Signature: 
Appendix D: Consent Form - Usability Test-Image Memory Test-July 1 st

\section{Research Personnel}

\begin{tabular}{|l|l|}
\hline Daniel LeBlanc & Dr. Robert Biddle \\
Principle Investigator & Faculty Sponsor \\
Carleton University & Carleton University \\
$(613) 435-1290$ & $(613) 520-2600$ Ext.6317 \\
dblanc@connect.carleton.ca & robert_biddle@carleton.ca \\
\hline
\end{tabular}

\section{Purpose}

The purpose of this usability test is to evaluate the visual patterns associated with images on a computer screen to investigate whether images can be used as passwords. We are trying to determine what patterns result from the characteristics of visual search in visual images. An eye tracker will be detecting eye movements during the session.

\section{Task Requirements}

We will ask you to complete a series of tasks that involve looking at various images on a computer screen for a preset amount of time. We will then ask that you fill out a short questionnaire, and at the conclusion of the testing session you will be asked to provide us with any suggestions you might have to improve the software.

\section{Duration and Locale}

Each session should take approximately 1 hour. You will be paid a $\$ 20$ honorarium, or given course credit, for your time. The testing will primarily take place at the HotLab located in room 210 SSRB.

\section{Potential Risk/Discomfort}

There will be no psychological or physical risk.

\section{Anonymity/Confidentiality}

All data that is collected will be held completely confidential. The data will only be made available to those people involved with this testing. Data will be coded for identification purposes.

\section{Right to Withdraw}

You have the right to withdraw at any time, without any explanation as to the reason for withdrawing from the testing. You will receive the $\$ 20$ honorarium, or course credit, even if you choose to withdraw from the study.

\section{Ethics Approval}

This study has been approved by the ethics committee for psychology research. 
If you have concerns about the ethics of this research, please contact Dr. Avi Parush. For other questions about the research, please contact Dr. Janet Mantler:

Dr. Janet Mantler

Chair, Department of Psychology

Carleton University

(613) 5202600 ext. 4173

psychchair@carleton.ca
Dr. Avi Parush

Chair, Carleton University Ethics Committee for

Psychological Research

Carleton University

(613) 5202600 ext . 6026

avi_parush@carleton.ca

\section{Signatures}

I have read and understand the above terms of testing and I understand the conditions of my participation. My signature indicates that I agree to participate in this experiment.

Participant's Name:

Participant's Signature:

Researcher's Name:

Researcher's Signature: 


\section{Research Personnel}

\begin{tabular}{|l|l|}
\hline Daniel LeBlanc & Dr. Robert Biddle \\
Principle Investigator & Faculty Sponsor \\
Carleton University & Carleton University \\
$(613) 435-1290$ & $(613) 520-2600$ Ext. 6317 \\
dblanc@connect.carleton.ca & robert_biddle@carleton.ca \\
\hline
\end{tabular}

Purpose

The purpose of this usability test is to evaluate the visual patterns associated with images on a computer screen to investigate whether images can be used as passwords. We are trying to determine what patterns result from the characteristics of visual search in visual images. An eye tracker will be detecting eye movements during the session.

\section{Task Requirements}

We will ask you to complete a series of tasks that involve looking at various images on a computer screen for a preset amount of time. We will then ask that you fill out a short questionnaire, and at the conclusion of the testing session you will be asked to provide us with any suggestions you might have to improve the software.

\section{Duration and Locale}

Each session should take approximately 1 hour. You will be paid a $\$ 10$ honorarium, or given course credit, for your time. The testing will primarily take place at the HotLab located in room 210 SSRB.

\section{Potential Risk/Discomfort}

There will be no psychological or physical risk.

\section{Anonymity/Confidentiality}

All data that is collected will be held completely confidential. The data will only be made available to those people involved with this testing. Data will be coded for identification purposes.

\section{Right to Withdraw}

You have the right to withdraw at any time, without any explanation as to the reason for withdrawing from the testing. You will receive the $\$ 10$ honorarium, or course credit, even if you choose to withdraw from the study.

\section{Ethics Approval}

This study has been approved by the ethics committee for psychology research. 
If you have concerns about the ethics of this research, please contact Dr. Avi Parush. For other questions about the research, please contact Dr. Anne Bowker:

Dr. Anne Bowker

Chair, Department of Psychology

Carleton University

(613) 5202600 ext. 2648

psychchair@carleton.ca
Dr. Avi Parush

Chair, Carleton University Ethics Committee for Psychological Research

Carleton University

(613) 5202600 ext . 6026

avi_parush@carleton.ca

Signatures

I have read and understand the above terms of testing and I understand the conditions of my participation. My signature indicates that I agree to participate in this experiment.

Participant's Name:

Participant's Signature:

Researcher's Name:

Researcher's Signature: 
Appendix D: Consent Form - Usability Test -Image Memory Test-SONA - July 1st

\section{Research Personnel}

\begin{tabular}{|l|l|}
\hline Daniel LeBlanc & Dr. Robert Biddle \\
Principle Investigator & Faculty Sponsor \\
Carleton University & Carleton University \\
$(613) 435-1290$ & $(613) 520-2600$ Ext. 6317 \\
dblanc@connect.carleton.ca & robert_biddle@carleton.ca \\
\hline
\end{tabular}

\section{Purpose}

The purpose of this usability test is to evaluate the visual patterns associated with images on a computer screen to investigate whether images can be used as passwords. We are trying to determine what patterns result from the characteristics of visual search in visual images. An eye tracker will be detecting eye movements during the session.

\section{Task Requirements}

We will ask you to complete a series of tasks that involve looking at various images on a computer screen for a preset amount of time. We will then ask that you fill out a short questionnaire, and at the conclusion of the testing session you will be asked to provide us with any suggestions you might have to improve the software.

\section{Duration and Locale}

Each session should take approximately 1 hour. You will be paid a $\$ 10$ honorarium, or given course credit, for your time. The testing will primarily take place at the HotLab located in room 210 SSRB.

\section{Potential Risk/Discomfort}

There will be no psychological or physical risk.

\section{Anonymity/Confidentiality}

All data that is collected will be held completely confidential. The data will only be made available to those people involved with this testing. Data will be coded for identification purposes.

\section{Right to Withdraw}

You have the right to withdraw at any time, without any explanation as to the reason for withdrawing from the testing. You will receive the $\$ 10$ honorarium, or course credit, even if you choose to withdraw from the study.

\section{Ethics Approval}

This study has been approved by the ethics committee for psychology research. 
If you have concerns about the ethics of this research, please contact Dr. Avi Parush. For other questions about the research, please contact Dr. Janet Mantler:

Dr. Janet Mantler

Chair, Department of Psychology

Carleton University

(613) 5202600 ext. 4173

psychchair@carleton.ca
Dr. Avi Parush

Chair, Carleton University Ethics Committee for Psychological Research Carleton University

(613) 5202600 ext . 6026

avi_parush@carleton.ca

Signatures

I have read and understand the above terms of testing and I understand the conditions of my participation. My signature indicates that I agree to participate in this experiment.

Participant's Name:

Participant's Signature:

Researcher's Name:

Researcher's Signature: 
This information will be held completely confidential.

(Please, do not put your name on this form!)

Age: years

Sex: (check one)<smiles>C1CCC1</smiles>
female

In what academic program are you enrolled?

At what level are you studying?<smiles>[14CH3][14CH2][14CH]1CCC[14CH2]1</smiles>

$\square$ Masters

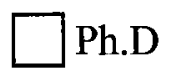

Other

What year of study are you in?

On a scale of 1 (novice) to 10 (expert), how would you rate yourself with respect to your computer skills?

How often do you browse the web?

Daily

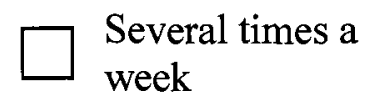

$\square$ Once a week Less than once a week

Approximately how many web sites do you visit that require a username and password?

Do you sometimes re-use the same password on different web sites? 
Are you concerned about the security of your passwords?

How do you decide if a web site is secure?

What criteria do you use for choosing a password? (Select more than one if appropriate)

It is easy for you to remember

It is difficult for others to guess

Other (please specify):
It is suggested by the system

It is the same as another password you currently have

Have you ever used a graphical password (using a picture to enter a password instead of typing in letters and numbers)? If so, where? 
This information will be held completely confidential.

(Please, do not put your name on this form!)

Age: years

Sex: (check one)

male

female

What is your occupation?

On a scale of 1 (novice) to 10 (expert), how would you rate yourself with respect to your computer skills?

How often do you browse the web?
Daily
Several times a week
$\square$ Once a week
Less than once a week

Approximately how many web sites do you visit that require a username and password?

Do you sometimes re-use the same password on different web sites? 
Are you concerned about the security of your passwords?

How do you decide if a web site is secure?

What criteria do you use for choosing a password? (Select more than one if appropriate)

$\square$ It is easy for you to remember

It is difficult for others to guess

$\square$ Other (please specify):
It is suggested by the system

It is the same as another password you currently have

Have you ever used a graphical password (using a picture to enter a password instead of typing in letters and numbers)? If so, where? 
Appendix F: Graphical Passwords Instructions

This is a usability test designed to test the ease of use, practicality, and security of graphical passwords. Please keep in mind that I am testing the effectiveness of the software and not you. I will ask you to complete a series of tasks and would like you to think aloud while you try to complete them. Some tasks are easy, while some are more difficult. Don't worry if you can't find the answer every time. The goal of this test is to evaluate how easy or difficult it is for people to use the software and to determine if these passwords are secure. The session will take about an hour.

We'll start with a practice session so that you can get used to entering graphical passwords. Then I'll ask you to set approximately 10 different graphical passwords. You will be asked to create, confirm, and then log in using the same graphical password for each image during the session.

Do you have any questions? 


\section{Appendix G: Image Memory Test Instructions}

This is a usability test designed to test the ease of use, practicality, and security of an image memory test. Please keep in mind that I am testing the effectiveness of the software and not you. I will ask you to complete a series of tasks and would like you to think aloud while you try to complete them. Some tasks are easy, while some are more difficult. Don't worry if you have difficulty with any of the tasks. The goal of this test is to evaluate whether the image tests are usable. The session will take about an hour.

We'll start with a practice session so that you can get used to remember image details. Then I'll ask you to view approximately 10 different images.

Do you have any questions? 


\section{Appendix H: Debriefing Form - Graphical Passwords}

The research we are conducting is part of a larger study examining the usability, practicality, and security of graphical passwords. These usability studies aim to assess the effectiveness of graphical passwords as alternatives to text-based passwords for systems requiring user authentication.

The usability test was designed to identify issues with using these programs, using eye gaze information to help us understand possible security implications.

The results of the usability test will be used to evaluate the practicality of graphical passwords and to make recommendations on how they can be improved. Your thoughts, comments, and opinions will be taken into consideration in making design recommendations. Thank you for participating in this usability test. Your time and effort are greatly appreciated!

If you have any further questions regarding this research, please contact:

\begin{tabular}{|l|l|}
\hline Daniel LeBlanc & Dr. Robert Biddle \\
Principle Investigator & Faculty Sponsor \\
Carleton University & Carleton University \\
$(613) 435-1290$ & $(613) 520-2600$ ext. 6317 \\
dblanc@connect.carleton.ca & robert_biddle@carleton.ca \\
\hline
\end{tabular}

If you have concerns about the ethics of this research, please contact:

\begin{tabular}{|l|l|}
\hline Dr. Anne Bowker & $\begin{array}{l}\text { Dr. Avi Parush } \\
\text { Chair, Carleton University Ethics }\end{array}$ \\
Chair, Department of Psychology & $\begin{array}{l}\text { Committee for Psychological Research } \\
\text { Carleton University }\end{array}$ \\
(613) 5202600 ext 2648 & (613) 5202600 ext. 6026 \\
psychchair@carleton.ca & avi_parush@carleton.ca \\
\hline
\end{tabular}


Appendix H: Debriefing Form - Graphical Passwords - July 1st

The research we are conducting is part of a larger study examining the usability, practicality, and security of graphical passwords. These usability studies aim to assess the effectiveness of graphical passwords as alternatives to text-based passwords for systems requiring user authentication.

The usability test was designed to identify issues with using these programs, using eye gaze information to help us understand possible security implications.

The results of the usability test will be used to evaluate the practicality of graphical passwords and to make recommendations on how they can be improved. Your thoughts, comments, and opinions will be taken into consideration in making design recommendations. Thank you for participating in this usability test. Your time and effort are greatly appreciated!

If you have any further questions regarding this research, please contact:

\begin{tabular}{|l|l|}
\hline Daniel LeBlanc & Dr. Robert Biddle \\
Principle Investigator & Faculty Sponsor \\
Carleton University & Carleton University \\
$(613) 435-1290$ & $(613) 520-2600$ ext.6317 \\
dblanc@connect.carleton.ca & robert_biddle@carleton.ca \\
\hline
\end{tabular}

If you have concerns about the ethics of this research, please contact:

\begin{tabular}{|l|l|}
\hline Dr. Janet Mantler & Dr. Avi Parush \\
Chair, Department of Psychology & Chair, Carleton University Ethics \\
Carleton University & Committee for Psychological Research \\
(613) 5202600 ext 4173 & (613) 5202600 ext. 6026 \\
psychchair@carleton.ca & avi_parush@carleton.ca \\
\hline
\end{tabular}




\section{Appendix I: Debriefing Form - Image Memory Test}

The research we are conducting is part of a larger study examining the usability, practicality, and security of using images instead of passwords for computer security.

The usability test was designed to identify issues with using these programs, using eye gaze information to help us understand possible security implications.

The results of the usability test will be used to evaluate the practicality of image passwords, and to make recommendations on how they can be improved. Your thoughts, comments, and opinions will be taken into consideration in making design recommendations. Thank you for participating in this usability test. Your time and effort are greatly appreciated!

If you have any further questions regarding this research, please contact:

\begin{tabular}{|l|l|}
\hline Daniel LeBlanc & Dr. Robert Biddle \\
Principle Investigator & Faculty Sponsor \\
Carleton University & Carleton University \\
$(613) 435-1290$ & $(613) 520-2600$ ext. 6317 \\
dblanc@connect.carleton.ca & robert_biddle@carleton.ca \\
\hline
\end{tabular}

If you have concerns about the ethics of this research, please contact:

\begin{tabular}{|l|l|}
\hline Dr. Anne Bowker & Dr. Avi Parush \\
Chair, Department of Psychology & Chair, Carleton University Ethics \\
Committee for Psychological Research \\
Carleton University & Carleton University \\
(613) 5202600 ext 2648 & (613) 5202600 ext. 6026 \\
psychchair@carleton.ca & avi parush@carleton.ca \\
\hline
\end{tabular}




\section{Appendix I: Debriefing Form - Image Memory Test-July 1st}

The research we are conducting is part of a larger study examining the usability, practicality, and security of using images instead of passwords for computer security.

The usability test was designed to identify issues with using these programs, using eye gaze information to help us understand possible security implications.

The results of the usability test will be used to evaluate the practicality of image passwords, and to make recommendations on how they can be improved. Your thoughts, comments, and opinions will be taken into consideration in making design recommendations. Thank you for participating in this usability test. Your time and effort are greatly appreciated!

If you have any further questions regarding this research, please contact:

\begin{tabular}{|l|l|}
\hline Daniel LeBlanc & Dr. Robert Biddle \\
Principle Investigator & Faculty Sponsor \\
Carleton University & Carleton University \\
$(613) 435-1290$ & $(613) 520-2600$ ext.6317 \\
dblanc@connect.carleton.ca & robert_biddle@carleton.ca \\
\hline
\end{tabular}

If you have concerns about the ethics of this research, please contact:

\begin{tabular}{|c|c|}
\hline Dr. Janet Mantler & Dr. Avi Parush \\
\hline Chair, Department of Psychology & $\begin{array}{l}\text { Chair, Carleton University Ethics } \\
\text { Committee for Psychological Research }\end{array}$ \\
\hline Carleton University & Carleton University \\
\hline (613) 5202600 ext 4173 & (613) 5202600 ext. 6026 \\
\hline psychchair@carleton.ca & avi_parush@carleton.ca \\
\hline
\end{tabular}


Appendix J: Post-Task Questionnaire - Graphical Passwords

1. I could easily create a graphical password

\begin{tabular}{|ccccccccccr|}
\hline \begin{tabular}{|l} 
Strongly \\
Disagree
\end{tabular} & & & & & & & & & & Strongly \\
1 & 2 & 3 & 4 & 5 & 6 & 7 & 8 & 9 & 10 \\
\hline
\end{tabular}

2. It was easy to decide what points to choose as part of my password

\begin{tabular}{|cccccccccr|}
\hline $\begin{array}{c}\text { Strongly } \\
\text { Disagree }\end{array}$ & & & & & & & & & $\begin{array}{r}\text { Strongly } \\
\text { Agree } \\
1\end{array}$ \\
\hline
\end{tabular}

3. It is easier to create text passwords than graphical passwords

\begin{tabular}{|c|c|c|c|c|c|c|c|c|c|}
\hline $\begin{array}{l}\text { Strongly } \\
\text { Disagree }\end{array}$ & & & & & & & & & $\begin{array}{l}\text { rongly } \\
\text { Agree }\end{array}$ \\
\hline $\begin{array}{c}\text { Disagree } \\
1\end{array}$ & 2 & 3 & 4 & 5 & 6 & 7 & 8 & 9 & 10 \\
\hline
\end{tabular}

4. With practice, I could quickly enter graphical passwords

\begin{tabular}{|cccccccccr|}
\hline \begin{tabular}{|l} 
Strongly \\
Disagree
\end{tabular} & & & & & & & & & Strongly \\
1 & 2 & 3 & 4 & 5 & 6 & 7 & 8 & 9 & 10 \\
\hline
\end{tabular}

5. I would trust a graphical password to protect my financial information.

\begin{tabular}{|cccccccccr|}
\hline \begin{tabular}{|l} 
Strongly \\
Disagree
\end{tabular} & & & & & & & & & Strongly \\
1 & 2 & 3 & 4 & 5 & 6 & 7 & 8 & 9 & 10 \\
\hline
\end{tabular}

6. I think that other people would choose different points than me for a graphical password.

\begin{tabular}{|ccccccccccr|}
\hline \begin{tabular}{|l} 
Strongly \\
Disagree
\end{tabular} & & & & & & & & & & Strongly \\
1 & 2 & 3 & 4 & 5 & 6 & 7 & 8 & 9 & 10 \\
\hline
\end{tabular}

7. Graphical passwords are quicker to use than text-based passwords

\begin{tabular}{|c|c|c|c|c|c|c|c|c|c|}
\hline $\begin{array}{l}\text { Strongly } \\
\text { Disagree }\end{array}$ & & & & & & & & & \multirow{2}{*}{$\begin{array}{r}\text { Strongly } \\
\text { Agree }\end{array}$} \\
\hline $\begin{array}{l}\text { Disagree } \\
1\end{array}$ & 2 & 3 & 4 & 5 & 6 & 7 & 8 & 9 & \\
\hline
\end{tabular}

8. Logging on using a graphical password was easy

\begin{tabular}{|cccccccccr|}
\hline $\begin{array}{c}\text { Strongly } \\
\text { Disagree } \\
1\end{array}$ & 2 & 3 & 4 & 5 & 6 & 7 & 8 & 9 & $\begin{array}{r}\text { Strongly } \\
\text { Agree } \\
1\end{array}$ \\
\hline
\end{tabular}

9. It was easy to click on the right points for my password

\begin{tabular}{|cccccccccr|}
\hline $\begin{array}{c}\text { Strongly } \\
\text { Disagree }\end{array}$ & & & & & & & & & Strongly \\
1 & 2 & 3 & 4 & 5 & 6 & 7 & 8 & 9 & 10 \\
\hline
\end{tabular}


10. I would use a graphical password

\begin{tabular}{|c|c|c|c|c|c|c|c|c|c|}
\hline \multirow{3}{*}{$\begin{array}{c}\text { Strongly } \\
\text { Disagree } \\
1 \\
\end{array}$} & & & & & & & & & \multirow{3}{*}{$\begin{array}{r}\text { Strongly } \\
\text { Agree } \\
10\end{array}$} \\
\hline & 0 & 9 & & 5 & 6 & & 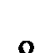 & & \\
\hline & 2 & 3 & 4 & 5 & 6 & 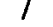 & 8 & 9 & \\
\hline
\end{tabular}

11. If I didn't log in to my account for a few weeks, I would still remember my graphical password

\begin{tabular}{|cccccccccr|}
\hline $\begin{array}{l}\text { Strongly } \\
\text { Disagree } \\
1\end{array}$ & 2 & 3 & 4 & 5 & 6 & 7 & 8 & 9 & Agree \\
1 & & & & & & & & & \\
\hline
\end{tabular}

12. It would be easier to remember 5 different text passwords than 5 different graphical passwords

\begin{tabular}{|llllllllrr|}
\hline $\begin{array}{l}\text { Strongly } \\
\text { Disagree } \\
1\end{array}$ & 2 & 3 & 4 & 5 & 6 & 7 & 8 & 9 & $\begin{array}{r}\text { Strongly } \\
\text { Agree } \\
10\end{array}$ \\
\hline
\end{tabular}

13. I chose points that are particularly memorable to me.

\begin{tabular}{|ccccccccccr|}
\hline $\begin{array}{c}\text { Strongly } \\
\text { Disagree }\end{array}$ & & & & & & & & & $\begin{array}{r}\text { Strongly } \\
\text { Agree }\end{array}$ \\
1 & 2 & 3 & 4 & 5 & 6 & 7 & 8 & 9 & 10 \\
\hline
\end{tabular}

14. Given the choice between a text password and a graphical password, I would choose a graphical password

\begin{tabular}{|lllllllllr|}
\hline $\begin{array}{l}\text { Strongly } \\
\text { Disagree } \\
1\end{array}$ & 2 & 3 & 4 & 5 & 6 & 7 & 8 & 9 & $\begin{array}{r}\text { Strongly } \\
\text { Agree }\end{array}$ \\
\hline
\end{tabular}

15. My accounts would be secure if protected by a graphical password

\begin{tabular}{|cccccccccr|}
\hline $\begin{array}{l}\text { Strongly } \\
\text { Disagree }\end{array}$ & & & & & & & & & $\begin{array}{r}\text { Strongly } \\
\text { Agree } \\
1\end{array}$ \\
\hline
\end{tabular}

16. Graphical passwords would be easy for attackers to guess

\begin{tabular}{|llllllllr|r|}
\hline $\begin{array}{l}\text { Strongly } \\
\text { Disagree } \\
1\end{array}$ & 2 & 3 & 4 & 5 & 6 & 7 & 8 & 9 & $\begin{array}{r}\text { Atrongly } \\
\text { Agree }\end{array}$ \\
\hline
\end{tabular}

17. Logging on using a graphical password was easier than with a text password

\begin{tabular}{|cccccccccc}
\hline \begin{tabular}{|l} 
Strongly \\
Disagree
\end{tabular} & & & & & & & & & Strongly \\
1 & 2 & 3 & 4 & 5 & 6 & 7 & 8 & 9 & 10 \\
\hline
\end{tabular}

18. Text passwords are more secure than graphical passwords

\begin{tabular}{|cccccccccr|}
\hline $\begin{array}{l}\text { Strongly } \\
\text { Disagree }\end{array}$ & & & & & & & & & Strongly \\
1 & 2 & 3 & 4 & 5 & 6 & 7 & 8 & 9 & 10 \\
\hline
\end{tabular}


19. I prefer text passwords to graphical passwords

\begin{tabular}{|c|c|c|c|c|c|c|c|c|c|}
\hline $\begin{array}{l}\text { Strongly } \\
\text { Disagree }\end{array}$ & & & & & & & & & $\begin{array}{l}\text { ongly } \\
\text { Agree }\end{array}$ \\
\hline 1 & 2 & 3 & 4 & 5 & 6 & 7 & 8 & 9 & 10 \\
\hline
\end{tabular}

20. Strangers and friends are equally likely to be able to guess my graphical password.

\begin{tabular}{|llllllllr|r|}
\hline \begin{tabular}{|l} 
Strongly \\
Disagree \\
1
\end{tabular} & 2 & 3 & 4 & 5 & 6 & 7 & 8 & 9 & $\begin{array}{r}\text { Strongly } \\
\text { Agree }\end{array}$ \\
\hline
\end{tabular}

21. It was easy to select a good graphical password

\begin{tabular}{|llllllllrr|}
\hline $\begin{array}{rlll}\text { Strongly } \\
\text { Disagree }\end{array}$ & & & & & & & & & Strongly \\
1 & 2 & 3 & 4 & 5 & 6 & 7 & 8 & 9 & 10 \\
\hline
\end{tabular}

22. The points I clicked on as my password are unlikely to have meaning to other people.

\begin{tabular}{|lllllllllr|r|}
\hline $\begin{array}{l}\text { Strongly } \\
\text { Disagree } \\
1\end{array}$ & 2 & 3 & 4 & 5 & 6 & 7 & 8 & 9 & Agrongly \\
\hline
\end{tabular}

23. Graphical passwords are easy to remember

\begin{tabular}{|ccccccccrrr|}
\hline $\begin{array}{l}\text { Strongly } \\
\text { Disagree } \\
1\end{array}$ & 2 & 3 & 4 & 5 & 6 & 7 & 8 & 9 & $\begin{array}{r}\text { Strongly } \\
\text { Agree }\end{array}$ \\
1
\end{tabular}

24. It would be easy for a friend or family member to guess my graphical password

\begin{tabular}{|ccccccccrr|}
\hline $\begin{array}{l}\text { Strongly } \\
\text { Disagree } \\
1\end{array}$ & 2 & 3 & 4 & 5 & 6 & 7 & 8 & 9 & $\begin{array}{r}\text { Strongly } \\
\text { Agree }\end{array}$ \\
\hline
\end{tabular}

25. Someone who knows me would be better at guessing my graphical password than a stranger.

\begin{tabular}{|llllllllrr|}
\hline $\begin{array}{l}\text { Strongly } \\
\text { Disagree }\end{array}$ & & & & & & & & & Strongly \\
1 & 2 & 3 & 4 & 5 & 6 & 7 & 8 & 9 & 10 \\
\hline
\end{tabular}

26. It would be easier for a friend to guess my text password than my graphical password

\begin{tabular}{|c|c|c|c|c|c|c|c|c|c|}
\hline $\begin{array}{l}\text { Strongly } \\
\text { Disagree }\end{array}$ & & & & & & & & & $\begin{array}{l}\text { rongly } \\
\text { Agree }\end{array}$ \\
\hline 1 & 2 & 3 & 4 & 5 & 6 & 7 & 8 & 9 & 10 \\
\hline
\end{tabular}

27. It was difficult to click on exactly the right points when entering my graphical password even though I knew my password

\begin{tabular}{|c|c|c|c|c|c|c|c|c|c|}
\hline $\begin{array}{l}\text { Strongly } \\
\text { Disagree }\end{array}$ & & & & & & & & & $\begin{array}{l}\text { rongly } \\
\text { Agree }\end{array}$ \\
\hline 1 & 2 & 3 & 4 & 5 & 6 & 7 & 8 & 9 & 10 \\
\hline
\end{tabular}


28. Everyone would select different graphical passwords for the same image

Strongly

Disagree

1

2

3

4

5

6

7

Strongly

Agree

29. Graphical passwords are too time-consuming

\begin{tabular}{|cccccccccr|}
\hline $\begin{array}{l}\text { Strongly } \\
\text { Disagree } \\
1\end{array}$ & 2 & 3 & 4 & 5 & 6 & 7 & 8 & 9 & $\begin{array}{r}\text { Strongly } \\
\text { Agree }\end{array}$ \\
\hline
\end{tabular}

30. If I was in a hurry, I would rather enter a text-based password than a graphical password

\begin{tabular}{|lllllllllrr|}
\hline $\begin{array}{l}\text { Strongly } \\
\text { Disagree }\end{array}$ & & & & & & & & & & Strongly \\
Agree & \\
1 & 2 & 3 & 4 & 5 & 6 & 7 & 8 & 9 & 10 \\
\hline
\end{tabular}

31. I believe I could guess other people's graphical passwords.

\begin{tabular}{|ccccccccrr|}
\hline $\begin{array}{c}\text { Strongly } \\
\text { Disagree } \\
1\end{array}$ & 2 & 3 & 4 & 5 & 6 & 7 & 8 & 9 & $\begin{array}{r}\text { Strongly } \\
\text { Agree } \\
10\end{array}$ \\
\hline
\end{tabular}

32. I would be happy if computer systems used graphical passwords instead of text passwords

\begin{tabular}{|llllllllrr|}
\hline $\begin{array}{l}\text { Strongly } \\
\text { Disagree } \\
1\end{array}$ & 2 & 3 & 4 & 5 & 6 & 7 & 8 & 9 & $\begin{array}{r}\text { Strongly } \\
\text { Agree } \\
10\end{array}$ \\
\hline
\end{tabular}

What strategy did you use for selecting your graphical passwords?

Which images were most helpful in selecting easy-to-remember passwords? Why?

How would your strategy change if you knew you had several graphical passwords to remember?

If you could pick your own images, what would you choose? Why? 
Appendix K: Post-Task Questionnaire - Image Memory Test

1. I could easily remember the images

\begin{tabular}{|rrrrrrrrrr|}
\hline $\begin{array}{l}\text { Strongly } \\
\text { Disagree } \\
1\end{array}$ & 2 & 3 & 4 & 5 & 6 & 7 & 8 & 9 & $\begin{array}{r}\text { Strongly } \\
\text { Agree }\end{array}$ \\
\hline
\end{tabular}

2. It was easy to decide how to remember the images

\begin{tabular}{|ccccccccccr}
\hline $\begin{array}{l}\text { Strongly } \\
\text { Disagree }\end{array}$ & & & & & & & & & $\begin{array}{r}\text { Strongly } \\
\text { Agree }\end{array}$ \\
1 & 2 & 3 & 4 & 5 & 6 & 7 & 8 & 9 & 10 \\
\hline
\end{tabular}

3. It is easier to remember words than image details

\begin{tabular}{|ccccccccrr|}
\hline $\begin{array}{l}\text { Strongly } \\
\text { Disagree } \\
1\end{array}$ & 2 & 3 & 4 & 5 & 6 & 7 & 8 & 9 & $\begin{array}{r}\text { Strongly } \\
\text { Agree } \\
10\end{array}$ \\
\hline
\end{tabular}

4. With practice, I could quickly remember these kinds of images

\begin{tabular}{|cccccccccrr|}
\hline \begin{tabular}{|l} 
Strongly \\
Disagree
\end{tabular} & & & & & & & & & Strongly \\
1 & 2 & 3 & 4 & 5 & 6 & 7 & 8 & 9 & 10 \\
\hline
\end{tabular}

5. I would trust an image memory test to protect my financial information

\begin{tabular}{|c|c|c|c|c|c|c|c|c|}
\hline Strongly & & & & & & & & Strongly \\
\hline Disagree & & & & & & & & Agree \\
\hline 1 & 2 & 3 & 4 & 5 & 6 & 7 & 8 & 10 \\
\hline
\end{tabular}

6. I think that other people would remember image details differently than me

\begin{tabular}{|c|c|c|c|c|c|c|c|c|c|}
\hline $\begin{array}{l}\text { Strongly } \\
\text { Disagree }\end{array}$ & & & & & & & & & $\begin{array}{l}\text { trongly } \\
\text { Agree }\end{array}$ \\
\hline $\begin{array}{c}\text { Disagree } \\
1\end{array}$ & 2 & 3 & 4 & 5 & 6 & 7 & 8 & 9 & $\begin{array}{l}\text { Agree } \\
10\end{array}$ \\
\hline
\end{tabular}

7. Image details are quicker to remember than words

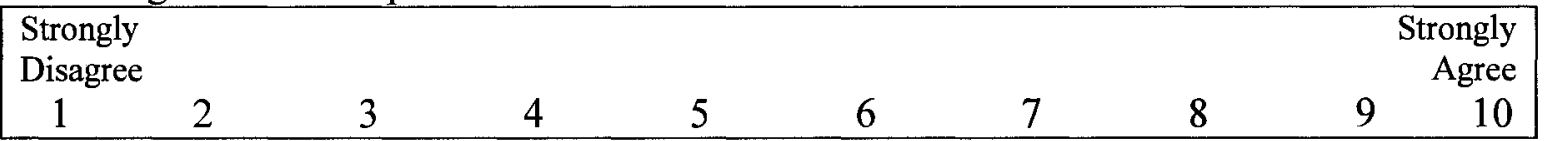

8. Recalling image details was easy

\begin{tabular}{|lllllllllr|}
\hline \begin{tabular}{|l} 
Strongly \\
Disagree
\end{tabular} & & & & & & & & & Strongly \\
1 & 2 & 3 & 4 & 5 & 6 & 7 & 8 & 9 & 10 \\
\hline
\end{tabular}

9. It was easy to identify the details to remember on the images

\begin{tabular}{|cccccccccr|}
\hline $\begin{array}{c}\text { Strongly } \\
\text { Disagree }\end{array}$ & & & & & & & & & $\begin{array}{r}\text { Strongly } \\
\text { Agree }\end{array}$ \\
1 & 2 & 3 & 4 & 5 & 6 & 7 & 8 & 9 & 10 \\
\hline
\end{tabular}


10. I would use an image memory test for computer security

\begin{tabular}{|c|c|c|c|c|c|c|c|c|c|}
\hline Strongly & & & & & & & & & \multirow{3}{*}{$\begin{array}{r}\text { Strongly } \\
\text { Agree } \\
10\end{array}$} \\
\hline Disagree & & & & & & & & & \\
\hline 1 & 2 & 3 & 4 & 5 & 6 & 7 & 8 & 9 & \\
\hline
\end{tabular}

11. If I didn't see them for a few weeks, I would still remember the image details

\begin{tabular}{|cccccccccr|}
\hline \begin{tabular}{|l} 
Strongly \\
Disagree
\end{tabular} & & & & & & & & & $\begin{array}{r}\text { Strongly } \\
\text { Agree } \\
1\end{array}$ \\
\hline
\end{tabular}

12. It would be easier to remember 5 different words than 5 different images

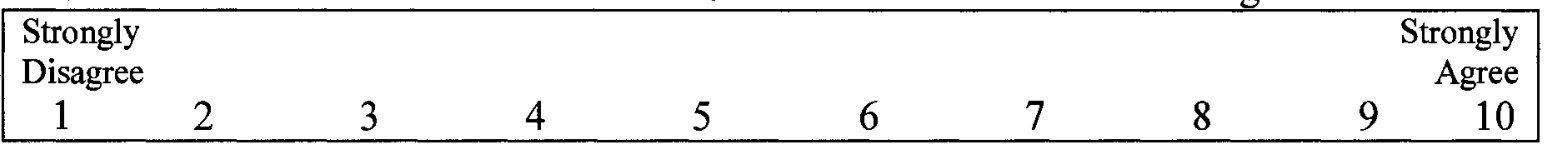

13. I chose image details that are particularly memorable to me

\begin{tabular}{|cccccccccr|}
\hline $\begin{array}{c}\text { Strongly } \\
\text { Disagree }\end{array}$ & & & & & & & & & \multicolumn{2}{r|}{$\begin{array}{r}\text { Strongly } \\
\text { Agree } \\
1\end{array}$} & 2 & 3 & 4 & 5 & 6 & 7 & 8 & 9 & 10 \\
\hline
\end{tabular}

14. Given the choice between remembering a word and an image, I would choose an image

\begin{tabular}{|ccccccccccr|}
\hline $\begin{array}{l}\text { Strongly } \\
\text { Disagree }\end{array}$ & & & & & & & & & & \multicolumn{2}{r}{$\begin{array}{r}\text { Strongly } \\
\text { Agree } \\
1\end{array}$} & 2 & 3 & 4 & 5 & 6 & 7 & 8 & 9 & 10 \\
\hline
\end{tabular}

15. My accounts would be secure if protected by an image memory test

\begin{tabular}{|c|c|c|c|c|c|c|c|c|c|}
\hline $\begin{array}{l}\text { Strongly } \\
\text { Disagree }\end{array}$ & & & & & & & & & $\begin{array}{l}\text { ongly } \\
\text { Agree }\end{array}$ \\
\hline $\begin{array}{c}\text { Disagree } \\
1\end{array}$ & 2 & 3 & 4 & 5 & 6 & 7 & 8 & 9 & 10 \\
\hline
\end{tabular}

16. Image memory tests would be easy for others to guess

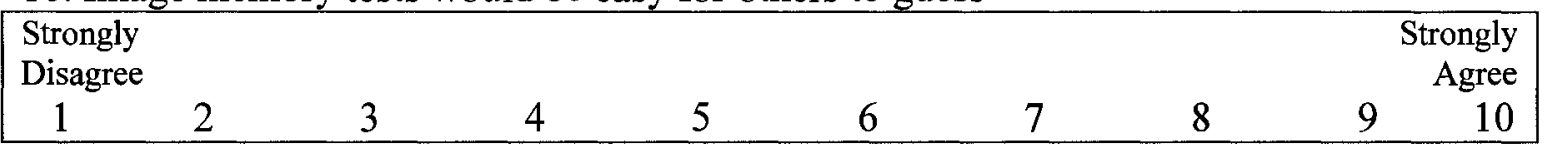

17. Logging on using an image test would be easier than with a text password

\begin{tabular}{|cccccccccr|}
\hline $\begin{array}{l}\text { Strongly } \\
\text { Disagree }\end{array}$ & & & & & & & & & \multicolumn{2}{r}{$\begin{array}{r}\text { Strongly } \\
\text { Agree } \\
1\end{array}$} & 2 & 3 & 4 & 5 & 6 & 7 & 8 & 9 & 10 \\
\hline
\end{tabular}

18. Text passwords are more secure than image memory tests

\begin{tabular}{|cccccccccr|}
\hline $\begin{array}{c}\text { Strongly } \\
\text { Disagree }\end{array}$ & & & & & & & & & \multicolumn{2}{r}{$\begin{array}{r}\text { Strongly } \\
\text { Agree } \\
1\end{array}$} & 2 & 3 & 4 & 5 & 6 & 7 & 8 & 9 & 10 \\
\hline
\end{tabular}


19. I prefer text passwords to image memory tests

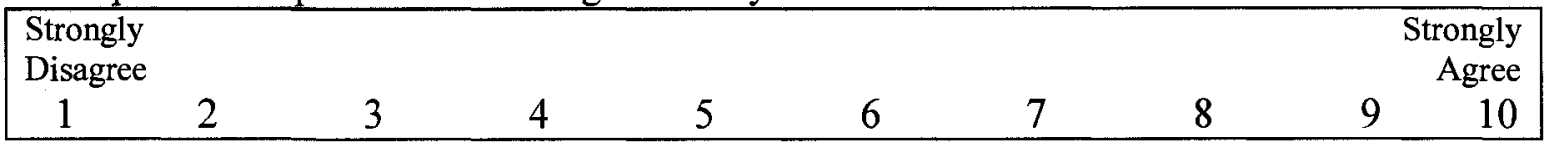

20. Strangers and friends are equally likely to be able to guess details I remember in an image

\begin{tabular}{|c|c|c|c|c|c|c|c|c|c|}
\hline $\begin{array}{l}\text { Strongly } \\
\text { Disagree }\end{array}$ & & & & & & & & \multicolumn{2}{|c|}{$\begin{array}{r}\text { Strongly } \\
\text { Agree }\end{array}$} \\
\hline $\begin{array}{c}\text { Disagree } \\
1\end{array}$ & 2 & 3 & 4 & 5 & 6 & 7 & 8 & 9 & $\begin{array}{c}\text { gree } \\
10\end{array}$ \\
\hline
\end{tabular}

21. It was easy to select image details that other people might not guess

\begin{tabular}{|c|c|c|c|c|c|c|c|c|c|}
\hline \multirow{2}{*}{$\begin{array}{l}\text { Strongly } \\
\text { Disagree } \\
1\end{array}$} & & & & & & & & & $\begin{array}{l}\text { trongly } \\
\text { Agree }\end{array}$ \\
\hline & 2 & 3 & 4 & 5 & 6 & 7 & 8 & 9 & 10 \\
\hline
\end{tabular}

22. The image details that I remembered most are unlikely to have meaning to other people

\begin{tabular}{|c|c|c|c|c|c|c|c|c|c|}
\hline $\begin{array}{l}\text { Strongly } \\
\text { Disagree }\end{array}$ & & & & & & & & & $\begin{array}{l}\text { ongly } \\
\text { igree }\end{array}$ \\
\hline 1 & 2 & 3 & 4 & 5 & 6 & 7 & 8 & 9 & 10 \\
\hline
\end{tabular}

23. Image details are easy to remember

\begin{tabular}{|cccccccccrr|}
\hline \begin{tabular}{|l} 
Strongly \\
Disagree
\end{tabular} & & & & & & & & & & Strongly \\
1 & 2 & 3 & 4 & 5 & 6 & 7 & 8 & 9 & 10 \\
\hline
\end{tabular}

24. It would be easy for a friend or family member to guess details I remembered in an image

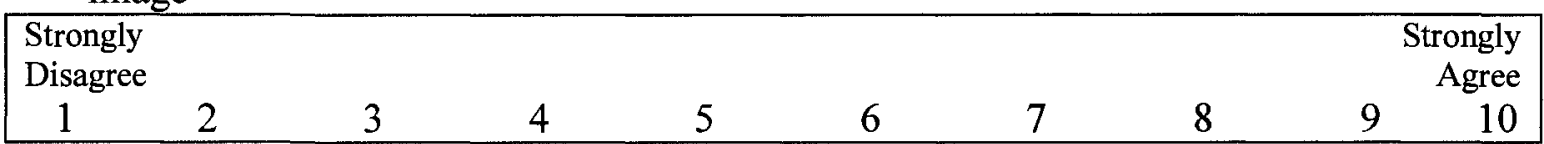

25. Someone who knows me would be better at guessing the details I remember in an image than a stranger

\begin{tabular}{|c|c|c|c|c|c|c|c|c|c|}
\hline $\begin{array}{l}\text { Strongly } \\
\text { Disarger }\end{array}$ & & & & & & & & & $\begin{array}{l}\text { ongly } \\
\text { Agree }\end{array}$ \\
\hline $\begin{array}{c}\text { Disagree } \\
1\end{array}$ & 2 & 3 & 4 & 5 & 6 & 7 & 8 & 9 & $\begin{array}{l}\text { Agree } \\
10\end{array}$ \\
\hline
\end{tabular}

26. It would be easier for a friend to guess my computer passwords than for details in an image that I remembered

\begin{tabular}{|ccccccccrrr|}
\hline $\begin{array}{l}\text { Strongly } \\
\text { Disagree }\end{array}$ & & & & & & & & & Strongly \\
1 & 2 & 3 & 4 & 5 & 6 & 7 & 8 & 9 & 10 \\
\hline
\end{tabular}


27. It was difficult to remember the details of images even though I remembered the image

\begin{tabular}{|cccccccccc|}
\hline $\begin{array}{l}\text { Strongly } \\
\text { Disagree } \\
1\end{array}$ & 2 & 3 & 4 & 5 & 6 & 7 & 8 & 9 & $\begin{array}{r}\text { Strongly } \\
\text { Agree }\end{array}$ \\
\hline
\end{tabular}

28. Everyone would remember different details for the same image

\begin{tabular}{|lllllllllr|}
\hline $\begin{array}{l}\text { Strongly } \\
\text { Disagree } \\
1\end{array}$ & 2 & 3 & 4 & 5 & 6 & 7 & 8 & 9 & $\begin{array}{r}\text { Strongly } \\
\text { Agree }\end{array}$ \\
\hline
\end{tabular}

29. Remembering images is too time-consuming

\begin{tabular}{|lllllllllr|}
\hline \begin{tabular}{|l} 
Strongly \\
Disagree \\
1
\end{tabular} & 2 & 3 & 4 & 5 & 6 & 7 & 8 & 9 & $\begin{array}{r}\text { Strongly } \\
\text { Agree }\end{array}$ \\
\hline
\end{tabular}

30. If I was in a hurry, I would rather remember a word than an image

\begin{tabular}{|lllllllllr|}
\hline $\begin{array}{l}\text { Strongly } \\
\text { Disagree }\end{array}$ & & & & & & & & & $\begin{array}{r}\text { Strongly } \\
\text { Agree } \\
1\end{array}$ \\
\hline
\end{tabular}

31. I believe I could guess details other people remember in images

\begin{tabular}{|c|c|c|c|c|c|c|c|c|c|}
\hline $\begin{array}{l}\text { Strongly } \\
\text { Disagree }\end{array}$ & & & & & & & & & $\begin{array}{l}\text { ongly } \\
\text { Agree }\end{array}$ \\
\hline 1 & 2 & 3 & 4 & 5 & 6 & 7 & 8 & 9 & 10 \\
\hline
\end{tabular}

32. I would be happy if computer systems used images instead of text passwords

\begin{tabular}{|c|c|c|c|c|c|c|c|c|c|}
\hline $\begin{array}{l}\text { Strongly } \\
\text { Disagree }\end{array}$ & & & & & & & & & $\begin{array}{l}\text { ongly } \\
\text { gree }\end{array}$ \\
\hline 1 & 2 & 3 & 4 & 5 & 6 & 7 & 8 & 9 & 10 \\
\hline
\end{tabular}

What strategy did you use for remembering your images?

Which images were most helpful in remembering details? Why?

How would your strategy change if you knew you had several images to remember?

If you could pick your own images, what would you choose? Why? 
Appendix L: Descriptive Statistics Tables

Overall times in seconds for all trials and images.

\begin{tabular}{l|cccccc}
\hline \multicolumn{1}{c|}{ Phase } & Minimum & $1^{\text {st }}$ Quartile & Median & Mean & $3^{\text {rd }}$ Quartile & Maximum \\
\hline Create & 4.819 & 17.07 & 27.61 & 34.23 & 43.64 & 318.2 \\
Confirm & 6.047 & 9.195 & 10.73 & 13.49 & 13.06 & 99.16 \\
Login & 6.703 & 9.352 & 10.64 & 11.68 & 13.13 & 22.69 \\
\hline
\end{tabular}

Overall times in seconds for trials 3 through 10.

\begin{tabular}{l|cccccc}
\hline \multicolumn{1}{c|}{ Phase } & Minimum & $1^{\text {st }}$ Quartile & Median & Mean & $3^{\text {rd }}$ Quartile & Maximum \\
\hline \multirow{2}{*}{ Create } & 4.891 & 16.69 & 26.2 & 30.33 & 39.18 & 108.9 \\
Confirm & 6.047 & 9.012 & 10.38 & 12.29 & 12.19 & 99.16 \\
Login & 6.703 & 9.281 & 10.42 & 11.37 & 12.94 & 22.69 \\
\hline
\end{tabular}

Times in seconds for trial 3.

\begin{tabular}{l|cccccc}
\hline \multicolumn{1}{c|}{ Phase } & Minimum & $1^{\text {st }}$ Quartile & Median & Mean & $3^{\text {rd }}$ Quartile & Maximum \\
\hline Create & 10.72 & 15.02 & 18.99 & 31.18 & 30.98 & 108.9 \\
Confirm & 7 & 8.906 & 10.26 & 12.35 & 13.78 & 26.28 \\
Login & 7 & 9.68 & 11.69 & 12.46 & 14.05 & 22.69 \\
\hline
\end{tabular}

Times in seconds for trial 4.

\begin{tabular}{l|cccccc}
\hline \multicolumn{1}{c|}{ Phase } & Minimum & $1^{\text {st }}$ Quartile & Median & Mean & $3^{\text {rd }}$ Quartile & Maximum \\
\hline Create & 8.656 & 16.29 & 22.34 & 26.71 & 33.96 & 64.33 \\
Confirm & 7.953 & 9.047 & 10.72 & 10.87 & 12.23 & 15.64 \\
Login & 8.734 & 9.984 & 10.67 & 11.09 & 11.48 & 16.12 \\
\hline
\end{tabular}

Times in seconds for trial 5.

\begin{tabular}{l|cccccc}
\hline \multicolumn{1}{c|}{ Phase } & Minimum & $1^{\text {st }}$ Quartile & Median & Mean & $3^{\text {rd }}$ Quartile & Maximum \\
\hline Create & 4.891 & 16.04 & 29.04 & 29.86 & 35.73 & 86.55 \\
Confirm & 6.047 & 9.695 & 10.59 & 13.58 & 13.23 & 31.77 \\
Login & 6.703 & 9.359 & 11.03 & 11.37 & 11.47 & 18.91 \\
\hline
\end{tabular}


Times in seconds for trial 6.

\begin{tabular}{l|cccccc}
\hline \multicolumn{1}{c|}{ Phase } & Minimum & $1^{\text {st }}$ Quartile & Median & Mean & $3^{\text {rd }}$ Quartile & Maximum \\
\hline Create & 13.53 & 15.8 & 34.54 & 33.34 & 42.8 & 70.52 \\
Confirm & 7.484 & 10.04 & 10.65 & 13.02 & 12.36 & 35.27 \\
Login & 6.844 & 9.531 & 11.41 & 11.98 & 12.98 & 20.48 \\
\hline
\end{tabular}

Times in seconds for trial 7.

\begin{tabular}{l|cccccc}
\hline \multicolumn{1}{c|}{ Phase } & Minimum & $1^{\text {st }}$ Quartile & Median & Mean & $3^{\text {rd }}$ Quartile & Maximum \\
\hline \multirow{2}{*}{ Create } & 14.05 & 17.55 & 31.37 & 32.92 & 44.92 & 69.58 \\
Confirm & 6.469 & 9.816 & 10.68 & 11.16 & 11.85 & 16.88 \\
Login & 6.797 & 9.234 & 10.66 & 11.19 & 12.17 & 19.55 \\
\hline
\end{tabular}

Times in seconds for trial 8.

\begin{tabular}{l|cccccc}
\hline \multicolumn{1}{c|}{ Phase } & Minimum & $1^{\text {st }}$ Quartile & Median & Mean & $3^{\text {rd }}$ Quartile & Maximum \\
\hline Create & 10.98 & 17.55 & 22.33 & 30.66 & 42.23 & 71.45 \\
Confirm & 6.297 & 8.551 & 10.74 & 16.36 & 12.55 & 99.16 \\
Login & 7.781 & 9.902 & 10.62 & 12 & 13.02 & 20.5 \\
\hline
\end{tabular}

Times in seconds for trial 9.

\begin{tabular}{l|cccccc}
\hline \multicolumn{1}{c|}{ Phase } & Minimum & $1^{\text {st }}$ Quartile & Median & Mean & $3^{\text {rd }}$ Quartile & Maximum \\
\hline Create & 16.78 & 17.4 & 26.34 & 30.32 & 34.83 & 70.97 \\
Confirm & 7.062 & 8.562 & 9.711 & 10.18 & 11.18 & 19.95 \\
Login & 7.062 & 8.984 & 9.969 & 10.48 & 12.34 & 15.67 \\
\hline
\end{tabular}

Times in seconds for trial 10.

\begin{tabular}{l|cccccc}
\hline \multicolumn{1}{c|}{ Phase } & Minimum & $1^{\text {st }}$ Quartile & Median & Mean & $3^{\text {rd }}$ Quartile & Maximum \\
\hline Create & 13.16 & 17.12 & 25.19 & 27.67 & 30.82 & 78.27 \\
Confirm & 6.062 & 8.574 & 9.688 & 10.82 & 11.39 & 21.89 \\
Login & 6.969 & 8.461 & 9.328 & 10.47 & 11.74 & 17.19 \\
\hline
\end{tabular}


Times in seconds for the Cars image.

\begin{tabular}{l|cccccc}
\hline \multicolumn{1}{c|}{ Phase } & Minimum & $1^{\text {st }}$ Quartile & Median & Mean & $3^{\text {rd }}$ Quartile & Maximum \\
\hline Create & 8.656 & 14.04 & 17.98 & 25.63 & 36.73 & 53.83 \\
Confirm & 6.047 & 8.824 & 10.07 & 10.54 & 11.39 & 19.95 \\
Login & 6.703 & 9.234 & 10.09 & 11.61 & 11.69 & 20.5 \\
\hline
\end{tabular}

Times in seconds for the Pool image.

\begin{tabular}{l|cccccc}
\hline \multicolumn{1}{c|}{ Phase } & Minimum & $1^{\text {st }}$ Quartile & Median & Mean & $3^{\text {rd }}$ Quartile & Maximum \\
\hline Create & 10.69 & 17.92 & 30.73 & 31.79 & 36.14 & 78.27 \\
Confirm & 8.328 & 9.715 & 10.4 & 11.54 & 11.66 & 21.89 \\
Login & 8.25 & 9.426 & 10.52 & 11.17 & 11.65 & 18.44 \\
\hline
\end{tabular}

Times in seconds for the Truck image.

\begin{tabular}{l|cccccc}
\hline \multicolumn{1}{c|}{ Phase } & Minimum & $1^{\text {st }}$ Quartile & Median & Mean & $3^{\text {rd }}$ Quartile & Maximum \\
\hline Create & 11.06 & 14.45 & 16.48 & 27.18 & 24.61 & 86.55 \\
Confirm & 6.469 & 8.461 & 9.703 & 10.49 & 10.86 & 23.95 \\
Login & 6.844 & 8.77 & 9.68 & 10.78 & 11.78 & 17.7 \\
\hline
\end{tabular}

Times in seconds for the Figureground image.

\begin{tabular}{l|cccccc}
\hline \multicolumn{1}{c|}{ Phase } & Minimum & $1^{\text {st }}$ Quartile & Median & Mean & $3^{\text {rd }}$ Quartile & Maximum \\
\hline Create & 4.891 & 14.88 & 20.35 & 23.67 & 31.02 & 50.5 \\
Confirm & 7.594 & 9.043 & 10.59 & 12.42 & 12.52 & 31.77 \\
Login & 7.062 & 8.863 & 9.594 & 10.01 & 10.26 & 18.91 \\
\hline
\end{tabular}

Times in seconds for the Topdown image.

\begin{tabular}{l|cccccc}
\hline \multicolumn{1}{c|}{ Phase } & Minimum & $1^{\text {st }}$ Quartile & Median & Mean & $3^{\text {rd }}$ Quartile & Maximum \\
\hline Create & 10.98 & 14.62 & 22.95 & 24 & 26.53 & 69.58 \\
Confirm & 6.062 & 7.562 & 9.602 & 9.717 & 11.11 & 16.88 \\
Login & 6.797 & 9.305 & 10.37 & 10.48 & 11.48 & 17.19 \\
\hline
\end{tabular}


Times in seconds for the Noise image.

\begin{tabular}{l|cccccc}
\hline \multicolumn{1}{c|}{ Phase } & Minimum & $1^{\text {st }}$ Quartile & Median & Mean & $3^{\text {rd }}$ Quartile & Maximum \\
\hline Create & 14.69 & 27.49 & 30.94 & 41.79 & 56.83 & 108.9 \\
Confirm & 9 & 11.34 & 11.86 & 18.11 & 14.81 & 99.16 \\
Login & 10.14 & 11.16 & 13.48 & 13.79 & 14.8 & 22.69 \\
\hline
\end{tabular}

Times in seconds for the Statue image.

\begin{tabular}{l|cccccc}
\hline \multicolumn{1}{c|}{ Phase } & Minimum & $1^{\text {st }}$ Quartile & Median & Mean & $3^{\text {rd }}$ Quartile & Maximum \\
\hline Create & 12.8 & 16.6 & 24.59 & 29.52 & 43.07 & 56.25 \\
Confirm & 6.859 & 8.109 & 9.625 & 12.48 & 11.07 & 35.27 \\
Login & 7 & 9.266 & 10.06 & 10.94 & 13.11 & 16.08 \\
\hline
\end{tabular}

Times in seconds for the Paperclips image.

\begin{tabular}{l|cccccc}
\hline \multicolumn{1}{c|}{ Phase } & Minimum & $1^{\text {st }}$ Quartile & Median & Mean & $3^{\text {rd }}$ Quartile & Maximum \\
\hline Create & 19.08 & 26.21 & 35.8 & 39.07 & 45.41 & 92.17 \\
Confirm & 7.062 & 10.57 & 11.8 & 13.05 & 12.38 & 27.92 \\
Login & 7.547 & 10.36 & 12.88 & 12.72 & 13.69 & 20.48 \\
\hline
\end{tabular}


Appendix M: Clearview Software Fixation Algorithm

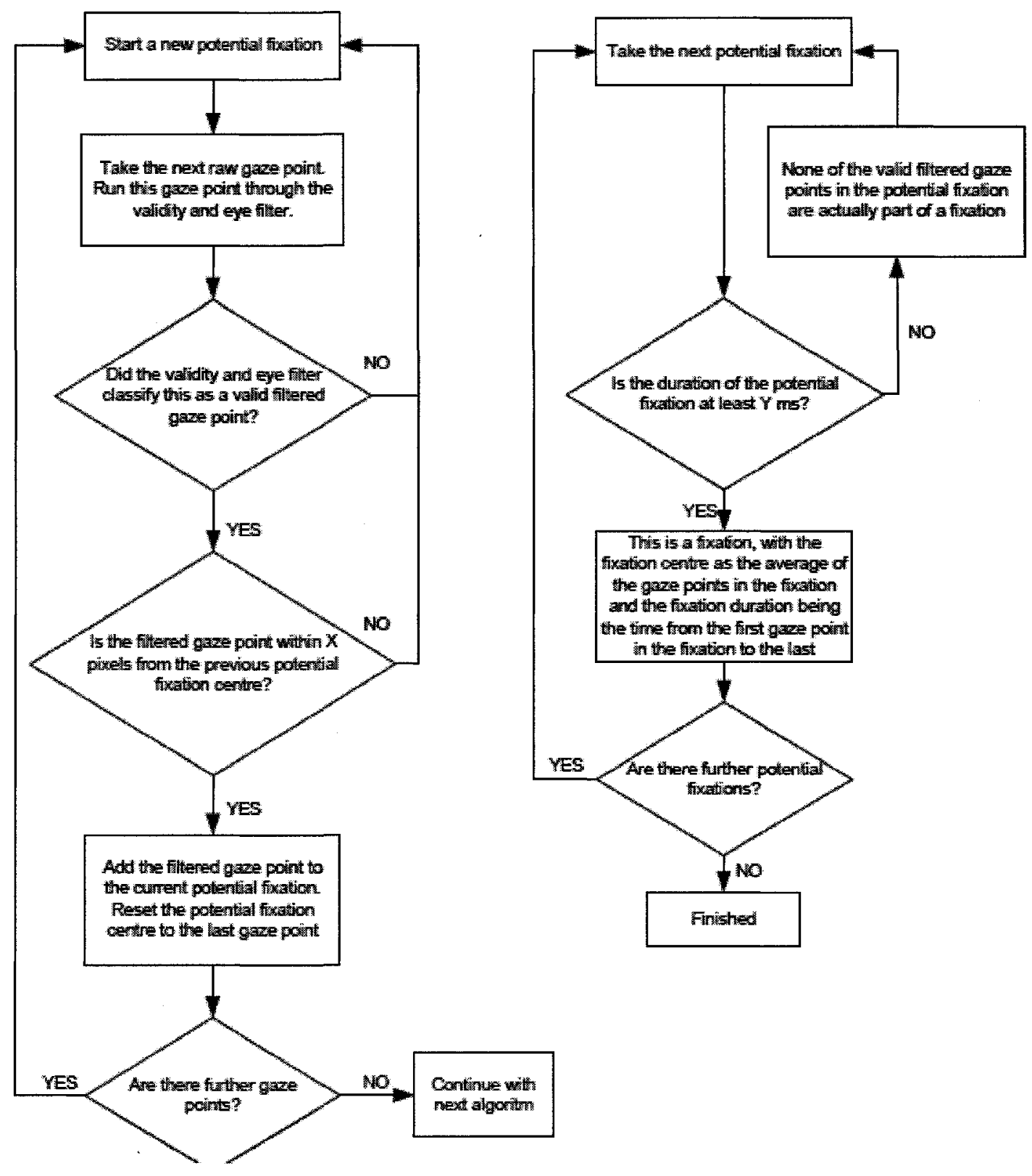




\section{Appendix N: Detailed Explanation of the JCross Statistic}

JCross, as defined by van Lieshout \& Baddeley (1999), is as follows: "Intuitively $\mathrm{J}_{i j}$ is a comparison between the distributions of the distances to the nearest type $j$ point, measured from (a) an arbitrary fixed point in $\mathrm{R}_{\mathrm{d}}$, and (b) a typical $i$ point. The denominator of (4) is the unconditional probability of the event that there is no type $j$ point within a distance $t$ of 0 .

As in the univariate case (Lieshout \& Baddeley, 1996) the value 1 is obtained when there is no spatial interaction: if $X_{i}$ are independent processes, then standard calculations give $J_{i j}=1$. However, having a $J_{i j}$-function taking value 1 everywhere should not be seen as a characterization of independence. In particular $J_{i i} \equiv 1$ is not a sufficient condition for $X_{i}$ to be a Poisson process (Bedford \& van der Berg, 1997). Similar remarks apply to the other statistics introduced in definition 1.

Values $J_{i j}>1$ can be interpreted as indicating inhibition (of type $j$ points by type $i$ points) since this is equivalent to $G_{i j}<F_{j}$, i.e. the presence of a type $i$ point decreases the probability of finding a type $j$ point nearby. Similarly, values less than 1 suggest positive association. (van Lieshout \& Baddeley, 1999, p. 514, paragraphs 2, 3, and 4)." 